\title{
Low Voltage Electrostatic Actuation and Displacement Measurement through Resonant Drive Circuit
}

\author{
by \\ Sangtak Park \\ A thesis \\ presented to the University of Waterloo \\ in fulfillment of the \\ thesis requirement for the degree of \\ Doctor of Philosophy \\ in \\ Systems Design Engineering
}

Waterloo, Ontario, Canada, 2011

(C) Sangtak Park 2011 


\section{AUTHOR'S DECLARATION}

I hereby declare that I am the sole author of this thesis. This is a true copy of the thesis, including any required final revisions, as accepted by my examiners.

I understand that my thesis may be made electronically available to the public. 


\begin{abstract}
An electrostatic actuator driven by conventional voltage control and charge control requires high actuation voltage and suffers from the pull-in phenomenon that limits its operation range, much less than its entire gap. To provide effective solutions to these problems, we present complete analytical and numerical models of various electrostatic actuators coupled with resonant drive circuits that are able to drive electrostatic actuators at much lower input voltage than that of conventional actuation methods and to extend their operation range beyond their conventional pull-in points in the presence of high parasitic capacitance. Moreover, in order to validate the analytical and numerical models of various electrostatic actuators coupled with the resonant drive circuits, we perform the experiment on the microplate and the micromirror coupled with the resonant drive circuit. For instance, using a high voltage amplifier, we manage to rotate the micromirror with sidewall electrodes by $6^{\circ}$ at $180 \mathrm{~V}$. However, using the resonant drive circuit, we are able to rotate the same micromirror by $6^{\circ}$ at much lower input voltage, $8.5 \mathrm{~V}$. In addition, the presented work also facilitates the stability analysis of electrostatic actuators coupled with the resonant drive circuits and provides how the effect of the parasitic capacitance can be minimized. For example, the resonant drive circuit placed within a positive feedback loop of a variable gain amplifier is able to extend the operation range much further even in the presence of very high parasitic capacitance. The resonant drive circuit with the proposed feedback controllers is also able to minimize the detrimental effects of the parasitic capacitance and to displace a parallel-plate actuator over its entire gap without the saddle-node bifurcation. Finally, we present a new displacement measurement method of electrostatic actuators coupled with the resonant drive circuits by sensing the phase delay of an actuation voltage with respect to an input voltage. This new measurement method allows us to easily implement feedback control into existent systems employing an electrostatic actuator without any modification or alteration to the electrostatic actuator itself. Hence, this research work presents the feasibility of electrostatic actuators coupled with the resonant drive circuit in various industrial and medical applications, in which the advantages of miniaturization, low supply voltage, and low power consumption are greatly appreciated.
\end{abstract}




\section{Acknowledgements}

I would like to show my gratitude to my supervisor, Prof. John T.W. Yeow and my committee members, Prof. Eihab Abdel-Rahman, Prof. Glenn R. Heppler, Prof. Behrad Khamesee, who have provided me with guidance, inspired me to work hard, encouraged me to stay on, and supported me to finish this research work.

I wish to express my sincere gratefulness to my colleagues and co-workers for their assistance: So-Ra Chung, Niraj Sinha, Jiazhi Ma, James Ting-Yu Lin, Keith Mo, Chau Do, Yanhui Bai, Mohsen Shahini, Andrew S. Logan, Yun Wang, Weijie Wan, Joyce Xinya Gao, Fred Sun, Rong Bai, Mehdi Shahini, Morteza Ahmadi, Qingsheng Kang, Albert I.H. Chen, Seungwoo Park, Shruti Nambiar, Manu Pallapa, Lawrence Wong, and Mahmoud Khater who have had these years together. I would like to acknowledge NSERC for my postgraduate scholarship, Waterloo Institute for Nanotechnology (WIN) for the fellowships, and CMC Microsystems for the fabrication service.

I am also very grateful to my friends and family for their advice, help, and invaluable guidance, which I cannot appreciate enough. 


\section{Dedication}

To my family members, who have nurtured me to be a better person, encouraged me to find the meaning of life, inspired me to be an engineer, taught me how to be committed, and endured this journey together with their love. 


\section{Table of Contents}

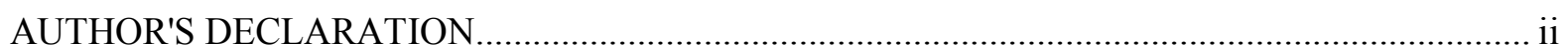

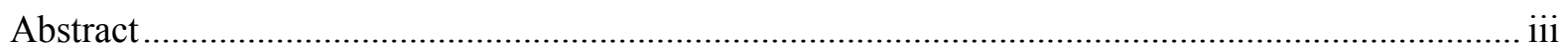

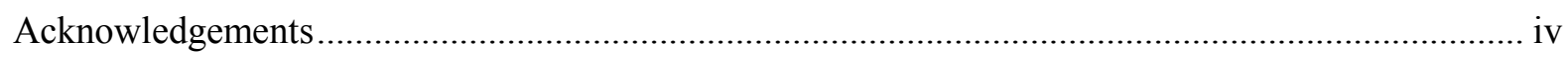

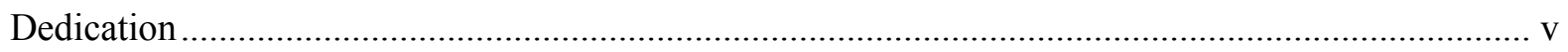

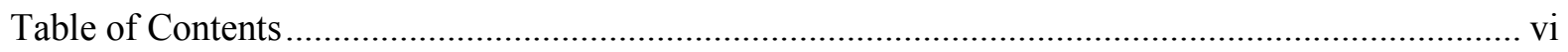

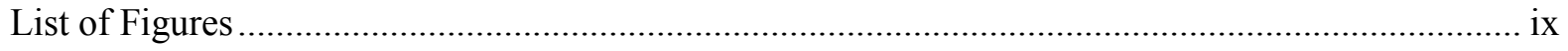

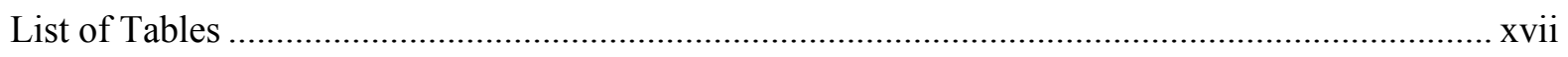

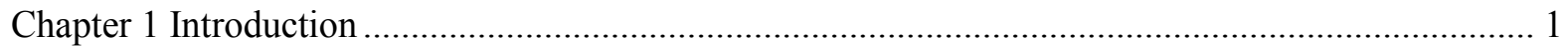

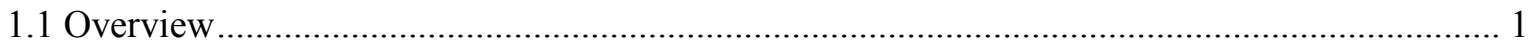

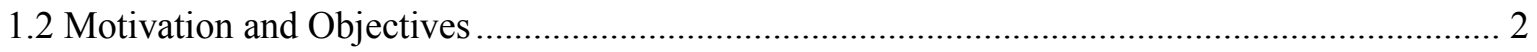

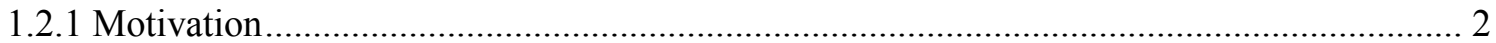

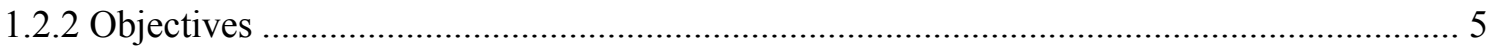

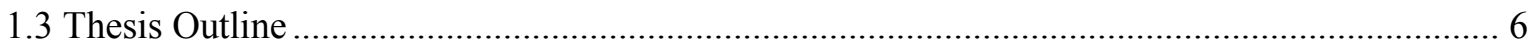

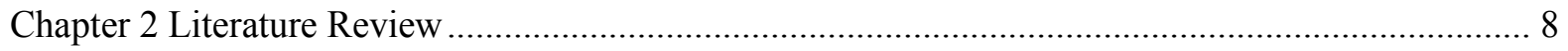

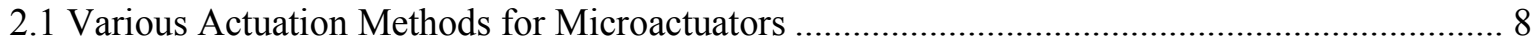

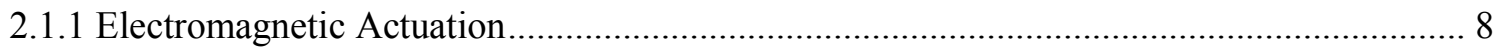

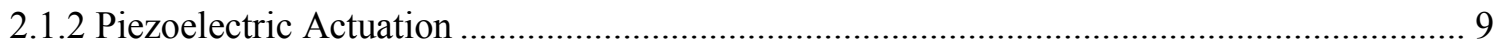

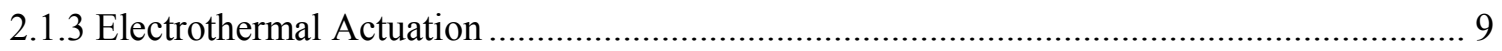

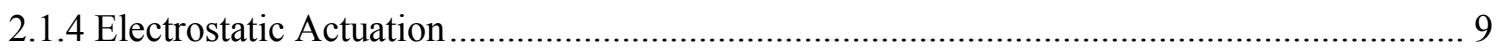

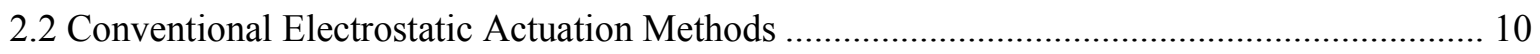

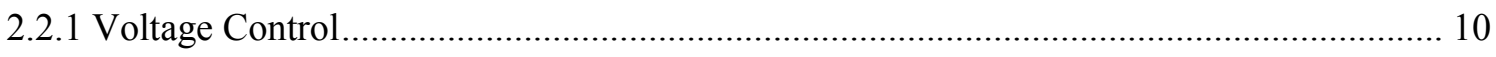

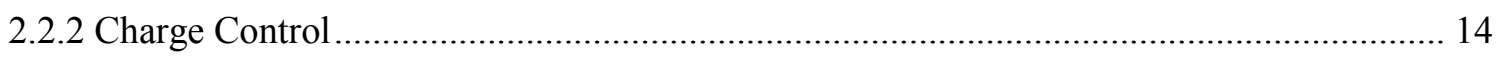

2.2.3 Voltage Control with a Feedback Capacitor ............................................................... 17

2.3 Electrostatic Actuation through Impedance Transformation ................................................ 19

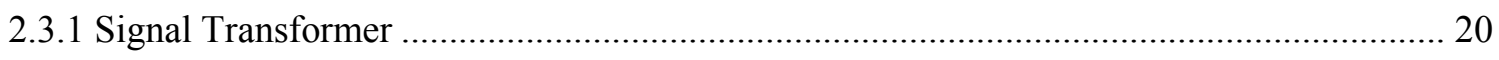

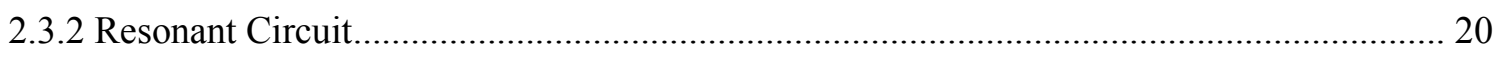

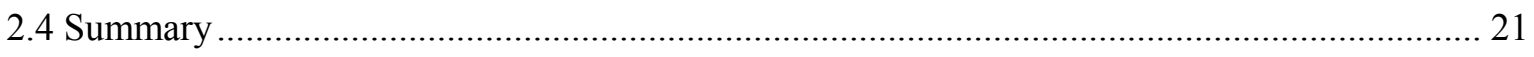

Chapter 3 Parallel-Plate Actuator Driven by Resonant Drive Circuit ............................................ 22

3.1 Analytical Model of Parallel-Plate Actuator Coupled with Resonant Drive Circuit Controlled

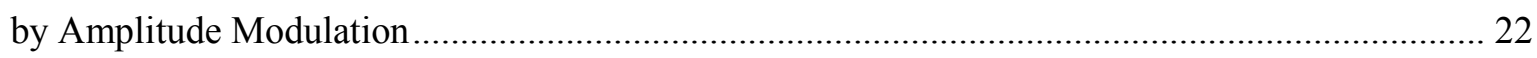


3.2 Stability Analysis of Parallel-Plate Actuator Driven by Resonant Drive Circuit Controlled by Amplitude Modulation

3.3 Analytical Model of Parallel-Plate Actuator Driven by Resonant Drive Circuit Controlled by

Frequency Modulation

3.4 Stability Analysis of Parallel-Plate Actuator Driven by Resonant Drive Circuit Controlled by

Frequency Modulation

3.5 Displacement Measurement of Parallel-Plate Actuator Coupled with Resonant Drive Circuit.34

3.5.1 Displacement Measurement through Phase Delay of Actuation Voltage with Respect to Input Voltage

3.5.2 Displacement Measurement through Phase Change between Input Current and Input Voltage .37

3.6 Numerical Model of Parallel-plate Actuator Coupled with Resonant Drive Circuit. .39

3.7 Experiment on Parallel Plates. 43

3.8 Summary 47

Chapter 4 Implementation of Resonant Drive Circuit 48

4.1 Unbalanced Resonant Drive Circuit 48

4.2 Balanced Resonant Drive Circuits. 49

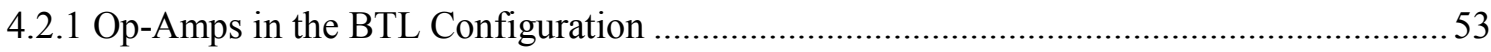

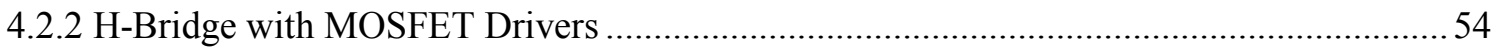

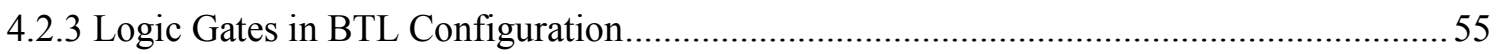

4.3 Resonant Drive Circuit with Enhanced Inherent Negative Feedback .....................................56

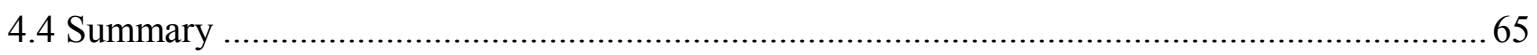

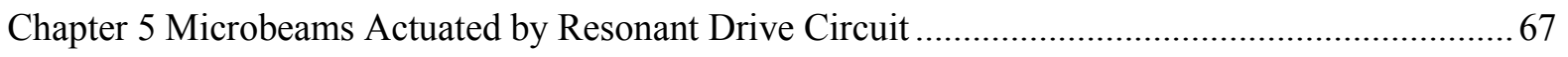

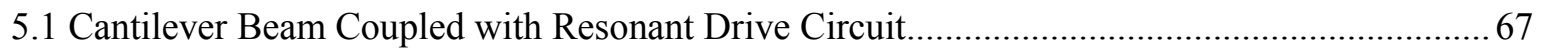

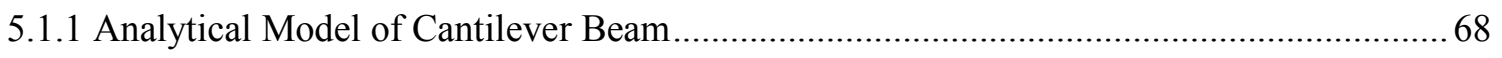

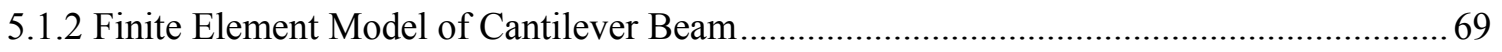

5.2 Fixed-Fixed Beam Driven by Resonant Drive Circuit ........................................................ 71

5.3 Microplate Supported by Two Microbeams and Actuated by Resonant Drive Circuit.............. 74

5.3.1 Finite Element Model of Microplate Guided by Two Microbeams …................................ 75

5.3.2 Experiment Results on Microplate Guided by Two Microbeams ...................................... 76

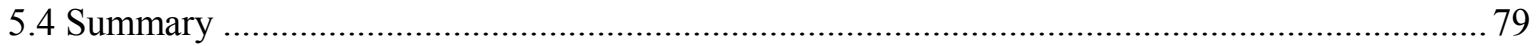

Chapter 6 Micromirror Coupled with Resonant Drive Circuits ......................................................... 80 
6.1 Analytical Model of Micromirror Driven by Voltage Control …....................................... 80

6.2 Analytical Model of Micromirror Coupled with Resonant Drive Circuits .............................. 84

6.3 Angular Displacement Measurement by Sensing Phase Delay between Actuation Voltage and

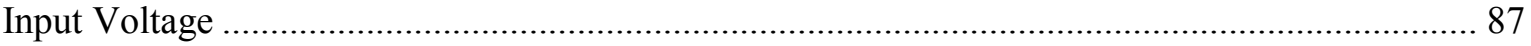

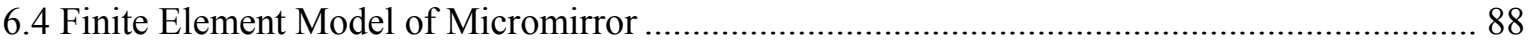

6.5 Experiment on Micromirror with Sidewall Electrodes Driven by Resonant Drive Circuit ....... 94

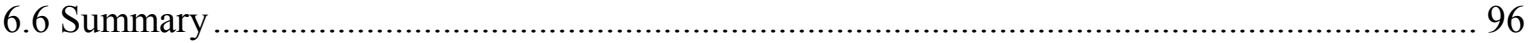

Chapter 7 Feedback Control with Resonant Drive Circuit ................................................................. 97

7.1 Nonlinear Feedback Control with Resonant Drive Circuit .................................................... 97

7.2 Linear Feedback Control with Resonant Drive Circuit.......................................................... 98

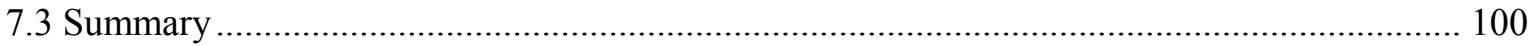

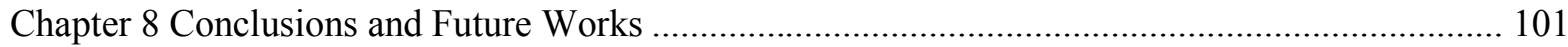

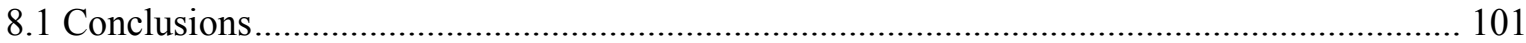

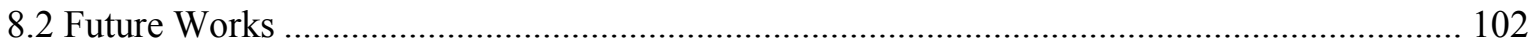

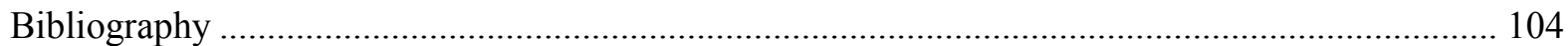




\section{List of Figures}

Figure 1.1 Examples of electrostatic actuators: (a) a top view of a microplate supported by a microbeam, (b) a false-color image of the same microplate acquired by a profilometer, (c) stacked torsional actuators post-processed with the displacement, and (d) a cantilever beam with a bottom electrode and its bonding pads

Figure 1.2 Simplified schematics of (a) a step-up transformer that provides impedance transformation over a wide range of frequencies up to $1 \mathrm{MHz}$ and (b) an LC resonant circuit delivering impedance transformation at its resonance.

Figure 2.1 Parallel-plate electrostatic actuator driven by voltage control represented by an ideal voltage source...... .11

Figure 2.2 Fixed points of the parallel-plate actuator driven by voltage control: the solid blue line represents the stable fixed points; the dotted blue line denotes the unstable fixed points; and the saddle-node bifurcation occurs at $1 / 3$ of the original gap at rest. .13

Figure 2.3 Numerical model of the parallel-plate actuator driven by voltage control described by the governing equation of Eq. 2.1. 14

Figure 2.4 Displacement of the parallel-plate actuator driven by voltage control: the pull-in voltage is $20.45 \mathrm{~V}$; and the pull-in point is $16.7 \mu \mathrm{m}$, which is equal to $1 / 3$ of the original gap, $50 \mu \mathrm{m}$.

Figure 2.5 Parallel-plate actuator driven by charge control; a charge source is represented by an ideal current source.

Figure 2.6 Fixed points of the parallel-plate actuator driven by charge control with various parasitic capacitances: $\mathbf{r}$ represents the ratio of the parasitic capacitance $\mathbf{C}_{\mathbf{p}}$ and the initial capacitance of the parallel-plate actuator $\mathbf{C}_{\mathbf{0}}$ at rest.

Figure 2.7 Saddle-node bifurcation points of the parallel-plate actuator driven by voltage control and charge control: the red dotted line represents the pull-in points of the parallel-plate actuator driven by voltage control; and the blue solid line denotes the pull-in points of the parallel-plate actuator driven by charge control with various parasitic capacitances.

Figure 2.8 Numerical model of the parallel-plate electrostatic actuator driven by charge control, which is created in Matlab/Simulink.

Figure 2.9 Displacement of the parallel-plate actuator driven by charge control as charge is accumulated in both the parallel-plate actuator and its parasitic capacitance.

Figure 2.10 Schematic of the parallel-plate actuator driven by voltage control with a feedback capacitor $\mathbf{C}_{\mathbf{f}}$ connected in series with a parallel-plate actuator and its parasitic capacitance....... 18 
Figure 2.11 Pull-in points of the parallel-plate actuator driven by voltage control with various sizes of feedback capacitors in series over a wide range of the parasitic capacitance.

Figure 2.12 Frequency responses of two resonant drive circuits: an unbalanced resonant drive circuit depicted by the dotted line and a balanced resonant drive circuit presented in the red solid line. 21

Figure 3.1 Schematic of the parallel-plate electrostatic actuator coupled with the resonant drive circuit driven by an AC voltage source without a DC bias 23

Figure 3.2 Fixed points of the parallel-plate actuator driven by conventional voltage control and the resonant drive circuit with the initial quality factor $\mathbf{Q}_{\mathrm{f}}=50$ and different parasitic capacitance: (a) $\mathbf{C}_{\mathbf{p}}=50 \mathbf{C}_{\mathbf{0}}$, (b) $\mathbf{C}_{\mathbf{p}}=100 \mathbf{C}_{\mathbf{0}}$, (c) $\mathbf{C}_{\mathbf{p}}=500 \mathbf{C}_{\mathbf{0}}$. The solid lines represent stable fixed points and the dotted lines depict unstable fixed points of the parallel-plate actuator. 28

Figure 3.3 Location of the saddle-node bifurcation of the parallel-plate actuator driven by the resonant drive circuit with the initial quality factor: (a) $\mathbf{Q}_{\mathrm{f}}=50$, (b) $\mathbf{Q}_{\mathrm{f}}=70$, (c) $\mathbf{Q}_{\mathrm{f}}=90$, (d) open-loop charge control represented by black dash-dot line. 28

Figure 3.4 Regions of the stable fixed points and the unstable fixed points of the parallel-plate actuator driven by the resonant drive circuit with the quality factor $\mathbf{Q}_{\mathrm{f}}$ of 50: the red solid line represents the location of the saddle-node bifurcation.

Figure 3.5 Fixed points of the parallel-plate actuator driven by the resonant drive circuit that is controlled by the frequency modulation with the parameters of $\mathbf{Q}_{\mathrm{f}}=50$ and $\overline{\mathbf{u}}=0.033$ : the solid lines represent the stable fixed points; and the dotted lines denote the unstable fixed points. .... 32

Figure 3.6 Fixed points of the parallel-plate actuator coupled with the resonant drive circuit controlled by the frequency modulation at various input voltages with $\mathbf{Q}_{\mathbf{f}}=50$ and $\mathbf{r}=100$.

Figure 3.7 Fixed points of the parallel-plate actuator coupled with the resonant drive circuit that is controlled by the frequency modulation at various quality factors with the fixed input voltage $\overline{\mathbf{u}}=0.03$ and $\mathbf{r}=200$.

Figure 3.8 Fixed points of the parallel-plate actuator coupled with the resonant drive circuit that is controlled by the frequency modulation in the presence of various parasitic capacitances with the fixed input voltage $\overline{\mathbf{u}}=0.03$ and the quality factor $\mathbf{Q}_{\mathrm{f}}=50$.

Figure 3.9 Envelope detector to measure the peak amplitude of the actuation voltage with an analogdigital converter (ADC) 35

Figure 3.10 Normalized displacement of the parallel-plate actuator in terms of the voltage gain with the initial quality factor $\mathbf{Q}_{\mathbf{f}}=50$, and the ratio of $\mathbf{C}_{\mathbf{p}}$ to $\mathbf{C}_{\mathbf{0}}, \mathbf{r}=25, \mathbf{r}=50, \mathbf{r}=100, \mathbf{r}=200$, and $\mathbf{r}=400$. 
Figure 3.11 Normalized displacement of the parallel-plate actuator in terms of the phase delay of the actuation voltage with reference to the input voltage in the case of the initial quality factor $\mathbf{Q}_{\mathbf{f}}=50$, and the ratio of $\mathbf{C}_{\mathbf{p}}$ to $\mathbf{C}_{\mathbf{0}}, \mathbf{r}=25, \mathbf{r}=50, \mathbf{r}=100, \mathbf{r}=200$, and $\mathbf{r}=400$. 36

Figure 3.12 Schematic of the phase detector to measure the phase delay of the actuation voltage with respect to the input signal.

Figure 3.13 Normalized displacement of the parallel-plate actuator in terms of the phase delay of the input current with reference to the input voltage in case of the initial quality factor $\mathbf{Q}_{\mathrm{f}}=50$, and the ratio of $\mathbf{C}_{\mathbf{p}}$ to $\mathbf{C}_{\mathbf{0}}, \mathbf{r}=25, \mathbf{r}=50, \mathbf{r}=100, \mathbf{r}=200$, and $\mathbf{r}=400$.

Figure 3.14 Block diagram of the numerical model of the parallel-plate actuator coupled with the resonant drive circuit that is created in Matlab/Simulink; its left side representing the resonant drive circuit and the right side depicting the parallel-plate actuator.

Figure 3.15 Block diagram of the numerical model of the simplified parallel-plate actuator coupled with the resonant drive circuit represented by Eq. 3.16 .

Figure 3.16 Step response of the parallel-plate actuator driven by the resonant drive circuit excited by the input voltage $\mathbf{u}=0.7 \mathrm{~V}$, which corresponds to the normalized input voltage $\overline{\mathbf{u}}=0.0342$; the actuation voltage is obtained from the numerical model in Figure 3.14; and the envelope of the actuation voltage is acquired from the numerical model in Figure 3.15.

Figure 3.17 Hysteresis of the parallel-plate actuator driven by the resonant drive circuit in case of the initial quality factor, $\mathbf{Q}_{\mathrm{f}}=50$ and the ratio of $\mathbf{C}_{\mathbf{p}}$ to $\mathbf{C}_{\mathbf{0}}, \mathbf{r}=100$; the fixed points represented by the blue thick solid line are obtained by the upward voltage sweep; and the fixed points denoted by the red solid line are acquired by the downward voltage sweep.

Figure 3.18 (a) Schematic of the experiment set-up with the parallel-plates controlled by the micropositioner and (b) the picture showing the parallel-plates with the micropositioner.....

Figure 3.19 Displacement measurement of the parallel-plates through (a) the voltage gain and (b) the phase delay of the actuation voltage with respect to the input voltage: the red solid line represents the analytical result, and the red solid boxes denote the measurement with $\mathbf{C}_{\mathbf{p}}=515 \mathrm{pF}$; the blue dashed line represents the analytical result, and the blue solid circles denote the measurement with $\mathbf{C}_{\mathbf{p}}=1015 \mathrm{pF}$.

Figure 3.20 Displacement measurement of the parallel-plates through the current gain (a) and phase delay of the current with respect to the input voltage (b); the red solid line representing the estimation and the red solid boxes denoting the measurement with $\mathbf{C}_{\mathbf{p}}=500 \mathrm{pF}$; the blue dashed 
line representing the estimation and the blue solid circles representing the measurement with $\mathbf{C}_{\mathbf{p}}=1000 \mathrm{pF}$

Figure 3.21 Screenshots of the oscilloscope that capture the input voltage (Ch 1, Yellow), the actuation voltage (Ch 2, Green), the current (Ch 4, Light Blue) through the resonant drive circuit in the main window, the Lissajous pattern of Ch 1 and Ch 2 in the $1^{\text {st }}$ window and the Lissajous pattern of $\mathrm{Ch} 1$ and $\mathrm{Ch} 4$ in the $2^{\text {nd }}$ window with $\mathbf{Q}_{\mathrm{f}}=32.35$ and $\mathbf{r}=58$ at (a) zero displacement, (b) $300 \mu \mathrm{m}$ displacement, (c) $600 \mu \mathrm{m}$ displacement, and (d) $900 \mu \mathrm{m}$ displacement 45

Figure 3.22 Screenshots of the oscilloscope that capture the input voltage (Ch 1, Yellow), the actuation voltage (Ch 2, Green), the current (Ch 4, Light Blue) through the resonant drive circuit in the main window, the Lissajous pattern of Ch 1 and $\mathrm{Ch} 2$ in the $1^{\text {st }}$ window and the Lissajous pattern of $\mathrm{Ch} 1$ and $\mathrm{Ch} 4$ in the $2^{\text {nd }}$ window with $\mathbf{Q}_{\mathrm{f}}=32.94$ and $\mathbf{r}=32$ at (a) zero displacement, (b) $300 \mu \mathrm{m}$ displacement, (c) $600 \mu \mathrm{m}$ displacement, and (d) $900 \mu \mathrm{m}$ displacement 46

Figure 4.1 Electrostatic actuator, represented by $\mathbf{C}_{\mathbf{m}}$, coupled with the unbalanced resonant drive circuit that is driven by a unity-gain amplifier U1, also known as a buffer.

Figure 4.2 the parallel-plate actuator coupled with the balanced resonant drive circuit driven by two voltage sources $180^{\circ}$ out of phase to each other.

Figure 4.3 Frequency responses of the unbalanced (the red dotted lines) and the balanced resonant drive circuits (the blue solid lines) implemented with the same electronic components. 50

Figure 4.4 Saddle nodes of the parallel-plate actuator driven by charge control circuit, the unbalanced and balanced resonant drive circuits with the same components: in terms of the ratio of $\mathrm{C}_{\mathrm{p}}$ to $\mathrm{C}_{0}$

Figure 4.5 Balanced resonant drive circuit excited by two op-amps in the BTL configuration: U1 is configured as a non-inverting amplifier with the voltage gain of 10.1, and U2 is arranged to be an inverting buffer to provide $180^{\circ}$.phase shift with respect to the output of U1.

Figure 4.6 Balanced resonant drive circuit driven by an H-bridge and MOSFET drivers: U1 is a MOSFET driver with the non-inverting output; U2 is a MOSFET driver that has an inverting output. 54

Figure 4.7 Balanced resonant drive circuit driven by two logic gates arranged in the BTL configuration: both logic gates have the push-pull output.

Figure 4.8 Impedance of the resonant drive circuit with respect to the input frequency: the input frequency is normalized with respect to the resonant frequency. 56 
Figure 4.9 (a) Unbalanced resonant drive circuit placed within a positive feedback loop of an op-amp that is configured as a non-inverting amplifier with the minimum gain of $\left(1+R_{4} / R_{3}\right)$ and (b) an unbalanced resonant drive circuit represented by $\mathrm{Z}$ in (a).

Figure 4.10 Voltage gain of the non-inverting amplifier in the previous figure in terms of the normalized impedance of $\mathrm{Z}$ with the condition of $\mathrm{R}_{1}=\mathrm{R}_{3}=\mathrm{R}_{4}$. .58

Figure 4.11 Op-amps, U1 and U2, configured as a non-inverting amplifier with both negative feedback and positive feedback loops implemented: (a) the negative feedback is stronger than the positive feedback; (b) the positive feedback is stronger than the negative feedback; (c) U1 and $\mathrm{U} 2$ are powered by $5 \mathrm{~V}$ and $-5 \mathrm{~V}$; and (d) an $1 \mathrm{~V}_{\mathrm{pp}}$ sinusoidal input signal without a DC bias at $10 \mathrm{~Hz}$. .59

Figure 4.12 the SPICE simulation result of the circuits in the previous figure: the red solid line, Vin, represents $1 \mathrm{~V}_{\mathrm{pp}}$ sinusoidal input signal at $10 \mathrm{~Hz}$; the green solid line, V3, denotes the output of $\mathrm{U} 1$ in the previous figure; and the blue solid line, $\mathrm{Vc}$, represents the output of U2 in the same figure.

Figure 4.13 Simplified schematic of the unbalanced resonant drive circuit placed within a positive feedback loop of an op-amp, the output voltage of which is equal to $\mathrm{V}_{\text {out }}$

Figure 4.14 Fixed points of the parallel-plate actuator driven by the resonant drive circuit arranged in the positive feedback loop of the non-inverting amplifier with the following parameters: $\mathrm{R}_{4}=2000 \Omega, \mathrm{R}_{\mathrm{p}}=2100 \Omega, \mathrm{Q}_{\mathrm{f}}=50$, and $\mathrm{R}_{1}=\mathrm{R}_{3}=100 \Omega$.

Figure 4.15 Fixed points of the parallel-plate actuator driven by the resonant drive circuit arranged in the positive feedback loop of the non-inverting amplifier with the following parameters: $\mathrm{R}_{4}=2000 \Omega, \mathrm{R}_{\mathrm{p}}=2100 \Omega, \mathrm{Q}_{\mathrm{f}}=50$, and $\mathrm{R}_{1}=\mathrm{R}_{3}=1000 \Omega$.

Figure 4.16 Saddle nodes of the parallel-plate actuator coupled with the unbalanced resonant drive circuit driven by the non-inverting amplifier implemented with both negative and positive feedback loops.

Figure 5.1 Isometric view of the cantilever beam, which is clamped on the right end, with its bottom electrode.

Figure 5.2 Displacement of the cantilever beam actuated by the resonant drive circuit (the red solid squares) and conventional voltage control (the blue solid circles) with the following parameters: the initial quality factor $\mathbf{Q}_{\mathrm{f}}=100$, the parasitic capacitance $\mathbf{C}_{\mathbf{p}}=100 \mathbf{C}_{\mathbf{0}}$ .70 
Figure 5.3 Cantilever beam post-processed with its displacement in the $\mathrm{z}$ axis while being actuated by the resonant drive circuit with the following parameters: the initial quality factor $\mathbf{Q}_{\mathrm{f}}=100$, the parasitic capacitance $\mathbf{C}_{\mathbf{p}}=50 \mathbf{C}_{\mathbf{0}}$, and the peak input voltage of $0.233 \mathrm{~V}$

Figure 5.4 Displacement of the cantilever beam actuated by the resonant drive circuit (the red solid squares) and conventional voltage control (the blue solid circles) with the following parameters: the initial quality factor $\mathbf{Q}_{\mathrm{f}}=100$, the parasitic capacitance $\mathbf{C}_{\mathbf{p}}=50 \mathbf{C}_{\mathbf{0}}$.

Figure 5.5 Isometric view of the fixed-fixed microbeam that has both ends clamped at the anchors. 71

Figure 5.6 Fixed-fixed microbeam post-processed with the displacement in the $\mathrm{z}$ axis while being actuated by the resonant drive circuit with the following parameters: the initial quality factor $\mathrm{Q}_{\mathrm{f}}=100$, the parasitic capacitance $\mathrm{C}_{\mathrm{p}}=100 \mathrm{C}_{0}$, and the peak input voltage $1.23 \mathrm{~V}$. 73

Figure 5.7 Stable fixed points of the fixed-fixed microbeam at various input voltages when driven by conventional voltage control and the resonant drive circuit with the initial quality factor $\mathrm{Q}_{\mathrm{f}}=100$ and the parasitic capacitance $\mathrm{C}_{\mathrm{p}}=100 \mathrm{C}_{0}$ 73

Figure 5.8 Isometric view of the microplate supported by a pair of microbeams attached to anchors. 74

Figure 5.9 (a) Top view of the microplate supported by two microbeams obtained through an optical microscope and (b) a false-color image of the same microplate acquired by a profilometer. ..... 74

Figure 5.10 Displacement profiles of the microplate supported by two microbeams at various actuation voltages: the pull-in voltage of the microplate is $6.84 \mathrm{~V}$. 75

Figure 5.11 Microplate post-processed with its displacement at the actuation voltage of $6.84 \mathrm{~V}$ before the pull-in occurs: the displacement of the center of the microplate is $0.409 \mu \mathrm{m}$. 76

Figure 5.12 Measured displacement and velocity of the microplate excited by the $8 \mathrm{~V}_{\mathrm{pp}}$ sinusoidal signal with the $\mathrm{DC}$ bias of $4 \mathrm{~V}$ at $1 \mathrm{~Hz}$ : $\mathrm{V}_{\mathrm{pi}}=6.57 \mathrm{~V}$ at $0.39 \mu \mathrm{m}$ and $\mathrm{V}_{\mathrm{po}}=1.867 \mathrm{~V}$.

Figure 5.13 Measured displacement and velocity of the microplate excited by the $8 \mathrm{~V}_{\mathrm{pp}}$ triangular signal with the DC bias of $4 \mathrm{~V}$ at $1 \mathrm{~Hz}: \mathrm{V}_{\mathrm{pi}}=6.57 \mathrm{~V}$ at $0.39 \mu \mathrm{m}$ and $\mathrm{V}_{\mathrm{po}}=1.867 \mathrm{~V}$.

Figure 5.14 Displacement of the microplate from the experiment and its FEA in COMSOL: the experiment result shows $\mathrm{V}_{\mathrm{pi}}=6.57 \mathrm{~V}$ at $0.39 \mu \mathrm{m}$; and FEA determines $\mathrm{V}_{\mathrm{pi}}=6.84 \mathrm{~V}$ at $0.41 \mu \mathrm{m} . .78$

Figure 5.15 Measured displacement of the microplate driven by the resonant drive circuit: the input voltage is $321 \mathrm{mV}_{\mathrm{pp}}$, the actuation voltage amplified by the resonant drive circuit is $12 \mathrm{~V}_{\mathrm{pp}}$, and the maximum displacement of the microplate is $271 \mu \mathrm{m}$.

Figure 6.1 (a) Isometric view of the micromirror suspended by a pair of serpentine torsion bars and (b) its cross-sectional view in the $x-y$ plane. .81 
Figure 6.2 (a) Isometric view of the serpentine torsion bar made of PolySi and (b) its top view: its thickness is same as that of the micromirror, $10 \mu \mathrm{m}$.

Figure 6.3 Fixed points of the micromirror driven by voltage control; the solid line represents the stable fixed points, and the dotted line denotes the unstable fixed points of the micromirror; and the saddle-node bifurcation occurs at $\boldsymbol{\varphi}=\mathbf{0 . 4 4}$.

Figure 6.4 (a) Schematics of an unbalanced resonant drive circuit and (b) a balanced resonant drive circuit: $\mathbf{C}_{\mathbf{m}}$ represents the capacitance of the micromirror; $\mathbf{C}_{\mathbf{p}}$ denotes the parasitic capacitance; $\mathbf{R}$ represents the parasitic resistance of the resonant drive circuit; $\mathbf{L}$ denotes the inductance of the inductor; and $\mathbf{u}_{1}(\mathbf{t})$ and $\mathbf{u}_{2}(\mathbf{t})$ represent two AC voltage sources being $180^{\circ}$ out of phase to each other.

Figure 6.5 Two op-amps in the BTL configuration: U1 is configured as a non-inverting amplifier with the voltage gain of 10.1 , and $\mathrm{U} 2$ is arranged to be an inverting buffer to provide $180^{\circ}$ phase shift with respect to the output of $\mathrm{U} 1$.

Figure 6.6 Fixed points of the micromirror driven by the resonant drive circuits with the quality factor $\mathbf{Q}_{\mathrm{f}}$ of 20: the dotted lines represent the unstable fixed points; the solid lines represent the stable fixed points; and the red lines and the blue lines denote the fixed points of the micromirror coupled with the balanced and unbalanced resonant drive circuits, respectively.

Figure 6.7 Diagram of a phase detector to measure the phase delay of the actuation voltage with respect to the input voltage.

Figure 6.8 Normalized angular displacement of the micromirror driven by the resonant drive circuits with $\mathbf{Q}_{\mathrm{f}}=20$ in terms of the phase delay of the actuation voltage: the red solid line, the blue dash-

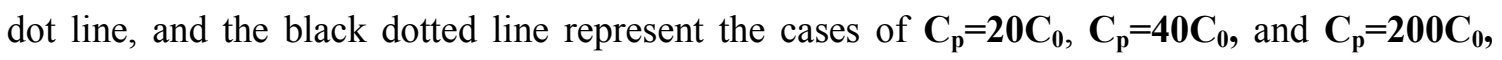
respectively. 88

Figure 6.9 Displacement of the centre and the edge of the micromirror in the y axis. 89

Figure 6.10 Displacement profiles of the micromirror at various actuation voltages: the dashed line, the dotted line, and the solid line represent the displacement profile of the micromirror when its actuation voltage are $75 \mathrm{~V}, 100 \mathrm{~V}$, and $125 \mathrm{~V}$, respectively. .90

Figure 6.11 Torsional stiffness of a pair of the serpentine torsion bars about the $\mathrm{z}$ axis and their linearized torsional stiffness. 91

Figure 6.12 Normal mode analysis of the micromirror suspended by a pair of the serpentine springs: (a) the $1^{\text {st }}$ mode at $543 \mathrm{~Hz}$; (b) the $2^{\text {nd }}$ mode at $3602 \mathrm{~Hz}$; (c) the $3^{\text {rd }}$ mode at $3915 \mathrm{~Hz}$; (d) the $4^{\text {th }}$ mode at $5271 \mathrm{~Hz}$; (e) the $5^{\text {th }}$ mode at $7218 \mathrm{~Hz}$; (f) the $6^{\text {th }}$ mode at $7525 \mathrm{~Hz}$..... 
Figure 6.13 (a) the micromirror meshed with hexahedral elements and (b) the micromirror postprocessed with the displacement in the y axis at the actuation voltage of $125 \mathrm{~V}$.

Figure 6.14 Fixed points of the micromirror with a pair of the serpentine torsion bars driven by the conventional voltage control: the dashed line represent the fixed points of the micromirror obtained through its FEM; the solid line denotes the stable fixed points of the micromirror derived from its analytical model; and the dotted line indicates the unstable fixed points. 92

Figure 6.15 Fixed points of the micromirror driven by the unbalanced resonant drive circuit with various parasitic capacitances: the dashed lines represent the fixed points of the micromirror obtained from its FEA; and the solid black lines denote the fixed points derived from its analytical model. 93

Figure 6.16 SEMS of the micromirror with sidewall electrodes: (a) the isometric view of the micromirror and (b) the top view of the micromirror showing its bottom electrodes and sidewall electrodes.

Figure 6.17 Mechanical angular displacement of the micromirror with sidewall electrodes driven by the resonant drive circuit and a high voltage amplifier.

Figure 7.1 Diagram of the nonlinear feedback controller implemented with the resonant drive circuit that drives the parallel-plate actuator.

Figure 7.2 Stable fixed points of the parallel-plate actuator coupled with the resonant drive circuit and the nonlinear controller in terms of the quality factor $\mathrm{Q}_{\mathrm{f}}=50$ and various parasitic capacitance. 98

Figure 7.3 Diagram of the feedback controller implemented with the resonant drive circuit that drives the parallel-plate actuator. 98

Figure 7.4 Fixed points of the parallel-plate actuator coupled with the resonant drive circuit and the feedback controller in terms of the quality factor $\mathbf{Q}_{\mathrm{f}}=50$, the feedback term of $\mathbf{a}=0.1$, and various parasitic capacitance.

Figure 7.5 Fixed points of the parallel-plate actuator coupled with the resonant drive circuit and the nonlinear controller in terms of the quality factor $\mathbf{Q}_{\mathrm{f}}=50$, the parasitic capacitance $\mathbf{C}_{\mathbf{p}}=200 \mathbf{C}_{\mathbf{0}}$ and various feedback multipliers. 100 


\section{List of Tables}

Table 2.1 Summary of Actuation Mechanism for Microactuators............................... 10

Table 2.2 Physical parameters of the parallel-plate actuator......................................

Table 3.1 Physical parameters used in the numerical model of the parallel-plate actuator coupled with the resonant drive circuit controlled by amplitude modulation ................................41

Table 4.1 Circuit parameters used to set the voltage gain of the non-inverting amplifier............63

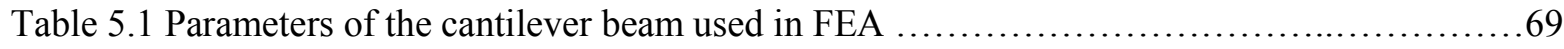

Table 5.2 Parameters of the fixed-fixed microbeam used in FEA ..............................72

Table 5.3 Physical properties of the microplate supported by two microbeams ....................75

Table 6.1 Parameters used for FEM of the micromirror coupled with the resonant drive circuit......90 



\section{Chapter 1 \\ Introduction}

\subsection{Overview}

The development of Micro-Electro-Mechanical Systems (MEMS) technology in the past decades has resulted in innovation to the automotive, communication and medical industries where size and mass reduction have improved performance of sensors and microactuators, such as accelerometers for inertial measurement, mass-flow sensors, and bio-chips for microfluidics, RF switches and automotive pressure sensors [1]. Popular MEMS devices for optical applications are optical switch arrays for communication [2]; optical coherence tomography for medical applications [3,5]; confocal laser scanning microscopy (CLSM) for obtaining high resolution images [4,5]; and the digital micromirror device for Digital Light Process (DLP) projection from Texas Instrument [6]. For example, a selection of MEMS electrostatic actuators is presented in Figure 1.1.

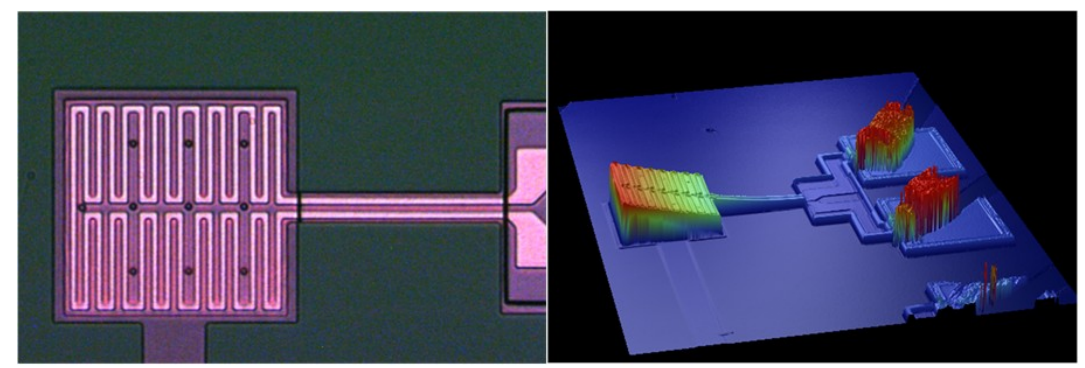

(a)

(b)

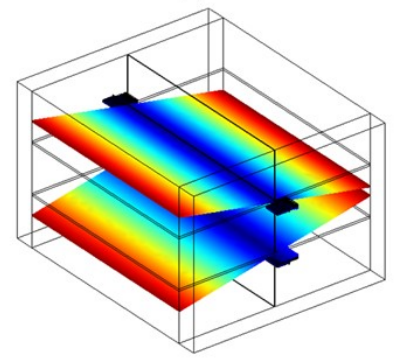

(c)

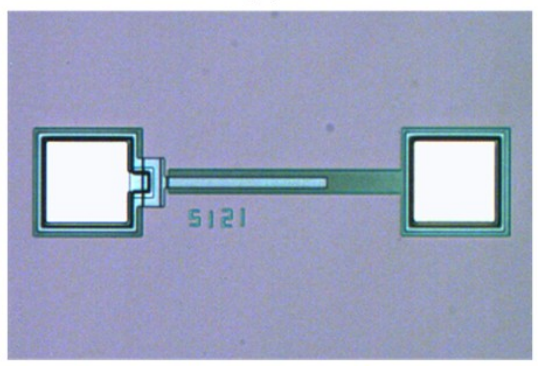

(d)

Figure 1.1 Examples of electrostatic actuators: (a) a top view of a microplate supported by a microbeam, (b) a false-color image of the same microplate acquired by a profilometer, (c) stacked torsional actuators post-processed with the displacement, and (d) a cantilever beam with a bottom electrode and its bonding pads

Electrostatic actuation is preferred for MEMS actuators because of its many advantages including fast response time and ease of integration and fabrication. The requirement of high actuation voltage is its major disadvantage. For instance, an electrostatically actuated micromirror can dramatically reduce the size and mass of a conventional endoscope. However, the high actuation voltage of a micromirror and its control circuit within an endoscope poses safety concerns because it is inserted into human cavities for therapeutic and diagnostic purposes. Hence, much research has been conducted to drive 
electrostatic actuators at low actuation voltage without a high voltage amplifier and a high voltage supply.

Another challenge for most electrostatic actuators is the pull-in phenomenon or the saddle-node bifurcation that limits their operation range. Thus, much research has been dedicated to extending an operation range of an electrostatic actuator beyond the pull-in point by the use of charge control $[7,8]$, voltage control with a feedback capacitor in series with an electrostatic actuator $[9,10]$, and implementation of a closed-loop control [11-13]. For instance, K.O. Owusu and F.L. Lewis [12] present that their tracking controller through feedback linearization can drive an electrostatic actuator over the entire gap in simulation, and D. Piyabongkarn et al. [13] use the inverse nonlinearity technique to displace a comb drive by $80 \%$ of its original gap. However, it is difficult to implement a feedback control due to noise and uncertainty in a displacement measurement.

Hence, we devise a new electrostatic actuation method of using impedance transformation circuitry to drive an electrostatic actuator beyond its pull-in point at low supply voltage. Further analysis and numerical simulation on resonant drive circuits presented in the next chapters confirm that the resonant drive circuits can reduce the supply voltage as well as drive an electrostatic actuator beyond its pull-in point even in the presence of high parasitic capacitance.

\subsection{Motivation and Objectives}

With the advent of MEMS technologies, many different microactuators have been developed with various actuation mechanisms including electromagnetic [14-17], electrostatic [18,19], electrothermal [20,21], and piezoelectric actuation [22-24]. Although electrostatic actuation is one of the most popular actuation methods, it requires high actuation voltage provided by a high voltage amplifier, which must be, in turn, powered by a high voltage power supply, it suffers from the pull-in phenomenon (saddle-node bifurcation), and it does not provide the displacement measurement capability.

\subsubsection{Motivation}

To provide reasonable solutions to these problems, we make use of impedance transformation. For example, most electrostatic actuators have high input impedance over the range of frequencies from $\mathrm{DC}$ to $10 \mathrm{MHz}$ and are considered as a variable capacitor, the capacitance of which varies from an order of $\mathrm{fF}$ to an order of $\mathrm{pF}$, depending on their geometric configuration. However, their operation requires high actuation voltage that cannot be provided by modern low voltage CMOS devices operating at $1.2 \mathrm{~V}$ to $5 \mathrm{~V}$. Hence, most electrostatic actuators have been driven by a high voltage amplifier that must be powered by a high voltage power supply. Although modern amplifiers and logic devices are not able to drive electrostatic actuators in a direct manner, they are capable of driving electrostatic actuators through impedance transformation circuitry by exploiting the feature of their low output impedance: electrostatic actuators require high actuation voltage ( $10 \mathrm{~V}$ to $300 \mathrm{~V})$, but sink very low current (a order of $\mathrm{pA}$ to an order of $\mathrm{nA}$ ); modern amplifiers and logic devices provide low output voltage $(0.8 \mathrm{~V}$ to $5 \mathrm{~V})$, but are able to source and sink high current (4 mA to $200 \mathrm{~mA})$, which are used to amplify low voltage into high actuation voltage through impedance transformation networks. 
A signal transformer, presented in Figure 1.2-(a), has been used for wideband impedance transformation, providing high actuation voltage from low input voltage over a wide range of frequencies. Driving an electrostatic actuator with a signal transformer is studied by H. Camon et al. [25]. L, T, and $\pi$ networks implemented with discrete components of resistors, capacitors, and inductors have been used for narrowband impedance transformation in many RF applications. Unlike a signal transformer, these networks work only at a specific frequency or over a very narrow range of frequencies. Hence, they are called narrowband impedance transformation networks. J. M. Kyynäräinen et al. [26] present their research regarding driving an electrostatic actuator through an L impedance transformation network that generates high actuation voltage from low AC input voltage through passive amplification at the resonance of the LC circuit depicted in Figure 1.2-(b). B. Cagdaser et al. [27] also use an LC tank circuit to drive an electrostatic actuator with low AC voltage signal at its electrical resonance. Hence, a constant voltage gain is achieved when a signal transformer is used for impedance transformation regardless of the frequencies of an input signal. However, their research does not provide an analytical model of the electrostatic actuator coupled with their LC drive circuits and is limited in the presence of low parasitic capacitance. Hence, we present and showcase the analytical and numerical model of the parallel-plate actuator driven by the resonant drive circuit to facilitate its study of nonlinear dynamics and to further understand how the quality factor of a resonant drive circuit compensates for the parasitic capacitance of an electrostatic actuator.

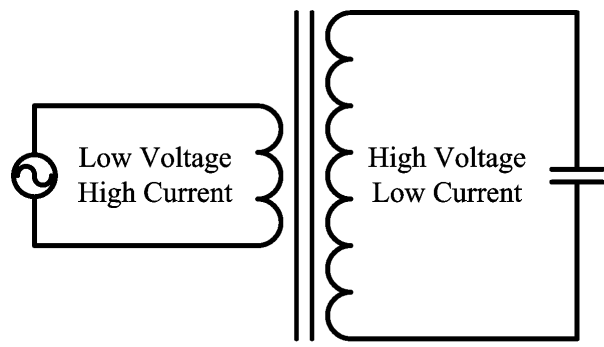

(a)

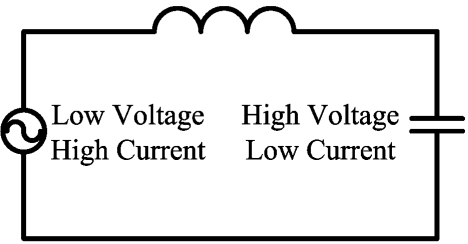

(b)

Figure 1.2 Simplified schematics of (a) a step-up transformer that provides impedance transformation over a wide range of frequencies up to $1 \mathrm{MHz}$ and (b) an $\mathrm{LC}$ resonant circuit delivering impedance transformation at its resonance

Another disadvantage of the electrostatic actuator is that it is difficult to sense its states such as displacement and velocity due to its small size and limitations of its fabrication methods. Much research has been dedicated to the displacement measurement of electrostatic actuators. For example, Robert C. Anderson et al. [28] has been using the capacitance measurement with additional sensing electrodes to measure the displacement of the electrostatic actuator. Van Spengen et al. [29] also use the capacitance measurement to detect a displacement of a comb drive. Hall et al. [30] use diffraction-based optical detection to measure the displacement of an accelerometer. Although this measurement method is immune to the parasitic capacitance and provides high sensitivity to the displacement, its fabrication is more complicated and expensive to be implemented. Ya'akobovitz et al. [31] employ optical microscopy and digital image correlation techniques to measure the in-plane displacement of a microbeam. Towfighian et al. $[32,33]$ use a vibrometer to measure the out-of-plane displacement of a microbeam for their nonlinear controller. However, an optical microscope or a 
vibrometer has an apparent disadvantage; its implementation into a small form factor is not possible in a micro scale. Hence, we present a simpler displacement measurement method by sensing the phase change of the actuation voltage or the current through a resonant drive circuit with respect to the input voltage without any modification or altercation on an electrostatic actuator itself.

For decades, a resonant circuit consisting of an inductor and a capacitor has been widely used in many industrial and biomedical applications: temperature measurement [34], chemical sensing [35], pressure monitoring [34,36,37], water content monitoring in construction materials [38], permittivity sensing in human tissues [34, 39], humidity sensing [34, 40], $\mathrm{pH}$ measurement [41], and strain sensing in structures [42]. In these applications, small change in the physical properties of interest causes deviation in the resonance frequency or impedance change within the resonant circuit. Through the measurement of these deviations in the resonance or impedance, the physical properties of interest have been monitored.

Since we use the resonant circuits to drive an electrostatic actuator, we can easily implement an additional measurement circuit to detect a change in a voltage gain or a phase delay with respect to an input voltage, which represents a change in a capacitance of an electrostatic actuator. In other words, as an electrostatic actuator displaces toward its bottom electrode, its capacitance increases, which reduces its resonant frequency. In turn, this increase in a capacitance results in less voltage gain and more phase delay in an actuation voltage and a current that flows through the resonant drive circuit. Through the measurement of this change in the amplitude or the phase of the voltage and the current with respect to the input voltage, we are able to measure a displacement of an electrostatic actuator without any sensing electrodes [28] or any optical diffraction measurement [30]. An envelope detector is implemented to measure a change in a voltage gain of the resonant drive circuits, and a phase detector is used to measure a phase change of an actuation voltage or a current through the resonant drive circuits with reference to an input AC voltage.

In summary, the electrostatic actuation through impedance transformation networks has considerable advantages over conventional voltage control and charge control as thus:

- It does not require a high voltage power supply or a high voltage amplifier to drive an electrostatic actuator.

- It consumes less power than conventional actuation methods do because of low quiescent current and low supply voltage.

- It does not need a heat sink or any other thermal control schemes due to its low power dissipation.

- It can be implemented in a small form factor, which provides considerable benefits to portable applications because of the same reasons.

- It is inherently safe and robust because there is no high voltage or high energy storage component within its control circuit.

- It is capable of extending the operation range beyond the pull-in point in the presence of high parasitic capacitance.

- It provides efficient methods to measure the displacement of an electrostatic actuator through the phase delay of an actuation voltage with reference to an input voltage. 
- It is able to improve the long-term reliability of electrostatic actuators by minimizing charge injection or hot-carrier injection to a dielectric layer to prevent the dielectric charging.

\subsubsection{Objectives}

To better understand the nonlinear dynamics of electrostatic actuators coupled with the resonant drive circuits, we derive their analytical models and develop their numerical and finite element models presented in the next chapters. Each chapter in this thesis is dedicated to each objective described in the following:

- Development of an analytical model of electrostatic actuators coupled with resonant drive circuits

Using the multiple scale methods [43], we derive an analytical model of electrostatic actuators driven by resonant drive circuits in order to understand how a resonant drive circuit extends the operation range of electrostatic actuators through its inherent negative feedback and to investigate how the parasitic capacitance of electrostatic actuators and the quality factor of the resonant drive circuit affect dynamics of electrostatic actuators.

- Development of a numerical model of electrostatic actuators coupled with resonant drive circuits

We also develop their numerical models in order to validate the analytical models of the electrostatic actuator driven by the resonant drive circuit and to investigate the transient behavior of the electrostatic actuator on a slow time scale because our interest resides on a nonlinear behavior of the electrostatic actuator.

- Development of a finite element model (FEM) of electrostatic actuators coupled with resonant drive circuits

In addition to the numerical models of electrostatic actuators driven by the resonant drive circuit, we develop FEMs of the electrostatic actuators, such as a cantilever beam, a fixed-fixed microbeam, a microplate supported by a pair of microbeams, and a micromirror suspended by two serpentine torsion bars, to validate each analytical model of various electrostatic actuators mentioned.

- Study on electrostatic actuators coupled with resonant drive circuits that are controlled by various modulation techniques

Various modulation schemes used in RF applications are capable of controlling the output voltage of the resonant drive circuit. Each modulation scheme appears to have distinctive impacts on how an electrostatic actuator responds to changes in its input voltage and input frequency. We derive the analytical models of the parallel-plate actuator coupled with the resonant drive circuit, which is controlled by amplitude modulation (AM) and frequency modulation (FM).

- Design and implementation of the resonant drive circuits

We also design and explore different types of the resonant drive circuits in order to optimize their characteristics and implementation depending on their control methods and configurations. In general, there are many ways to implement the resonant drive circuits, all of which require an $\mathrm{AC}$ voltage source. We need to control either the amplitude or the frequency of the AC voltage source in order to control the displacement of an electrostatic actuator. 
- Study on the displacement measurement method through the phase delay

We investigate how to measure the displacement of an electrostatic actuator when it is driven by the resonant drive circuits. As noted previously, we can measure either a change in a voltage gain or a phase delay of an actuation voltage with respect to an input $\mathrm{AC}$ voltage signal in order to determine the displacement. We design and present two displacement measurement circuits while discussing advantages and disadvantages of each measurement circuit.

- Development of nonlinear feedback control methods with the resonant drive circuits

Using the displacement measurement circuits, we can easily implement a feedback control on an electrostatic actuator without modifying an electrostatic actuator itself. Hence, we design and present nonlinear feedback control methods, which can extend the operation range of an electrostatic actuator that is coupled with the resonant drive circuits and improve the stability of an electrostatic actuator under disturbance.

\subsection{Thesis Outline}

This thesis is organized into eight chapters and each chapter is also divided into subsections, each focusing on a specific topic in detail, according to its contents and relevance to topics. For readability, each chapter is prepared to be self-contained as much as possible.

In Chapter 2, we provide the background knowledge and previous research on the various actuation methods for microactuators fabricated by MEMS technologies: electrostatic, electromagnetic, piezoelectric, and electrothermal actuation. Moreover, more details on conventional electrostatic actuation methods are presented and discussed: each actuation method is reviewed in terms of its advantages and disadvantages through an analytical and numerical model of a parallel-plate electrostatic actuator.

In Chapter 3 we introduce the resonant drive circuit that is capable of driving most electrostatic actuators at low supply voltage and extending the operation range of electrostatic actuators in the presence of high parasitic capacitance. The analytical and numerical models of the parallel-plate actuator coupled with the resonant drive circuit are derived and presented to evaluate the performance of the resonant drive circuit in comparison to that of conventional electrostatic actuation. Two actuation methods implemented with the resonant drive circuit are also presented in this chapter: amplitude control and frequency control. Furthermore, the displacement measurement through the phase delay of the actuation voltage signal with reference to the input voltage signal is introduced.

In Chapter 4, we present the different types of the resonant drive circuits, which provide impedance transformation and provide explanation on how the resonant drive circuits can be implemented and optimized in terms of the voltage gain and the robustness to the saddle-node bifurcation.

In Chapter 5, we create and present the finite element models of various electrostatic actuators coupled with the resonant drive circuits: a cantilever beam, a fixed-fixed microbeam, and a microplate supported by a pair of microbeams. We demonstrate to validate the feasibility of the resonant drive circuits on various electrostatic actuators. 
In Chapter 6, we present the analytical model and the finite element model of the micromirror supported by a pair of serpentine springs, when the micromirror is driven by either a voltage control or the resonant drive circuit in order to compare its static responses at various input voltages. Moreover, using the analytical model of the micromirror coupled with the resonant drive circuits, we derive the operation range of the micromirror in terms of the quality factor of the resonant drive circuits and the parasitic capacitance of the micromirror.

In Chapter 7, we introduce the resonant drive circuit with closed-loop control that utilizes the displacement measurement techniques presented in Chapter 3. As previously mentioned, linear control schemes are not of much use in stabilizing an electrostatic actuator at its pull-in point, and the displacement measurement is not possible to be implemented without modification of an electrostatic actuator itself. Hence, we present nonlinear control schemes capable of driving an electrostatic actuator at low input voltages and capable of extending the operation range beyond its pull-in point while making use of the displacement measurement method introduced in Chapter 3.

Finally, in Chapter 8, we summarize the contributions, providing conclusions and future works related to electrostatic actuator driven by the resonant drive circuits. 


\section{Chapter 2}

\section{Literature Review}

Microactuators fabricated by MEMS technology have been proven to be critical components in many industrial and biomedical applications such as laser scanning displays, optical switch matrix, and biomedical imaging systems. In this chapter, various actuation mechanisms for microactuators are presented, described and compared in terms of actuation voltage, power consumption, and fabrication methods.

This chapter is organized as follows. In Section 2.1, various actuation methods, which are used to drive microactuators fabricated by MEMS technologies, are reviewed and discussed in terms of their advantages and disadvantages. In Section 2.2, more details on electrostatic actuation methods are reviewed and presented along with their analytical and numerical models. In Section 2.3, electrostatic actuation methods through impedance transformation are also reviewed and presented.

\subsection{Various Actuation Methods for Microactuators}

Microactuators have been fabricated by various fabrication processes, such as Poly Multi-User MEMS Processes (PolyMUMPs), Silicon-On-Insulator MUMPs (SOIMUMPs), Metal MUMPs, and custom processes. Different fabrication processes allow us to build microactuators that can be actuated in different manners. For example, electromagnetic actuation requires ferromagnetic materials, usually $\mathrm{Ni}$, to be deposited on microactuators, and this process is not compatible with standard CMOS processes. Hence, a custom process is usually employed to build electromagnetic actuators. Even though many unique actuation methods are developed, the conventional actuation methods are electromagnetic, piezoelectric, electrothermal, and electrostatic actuation.

\subsubsection{Electromagnetic Actuation}

Currently, electromagnetic actuation provides more displacement than other actuation methods do. For instance, Takayuki Iseki et al. [14] implement electromagnetic actuation to displace a micromirror, achieving a large angular scan angle of $8^{\circ}$ at $0.75 \mathrm{~mA}$, while a micromirror shows a linear response to the applied current. However, its implementation is limited by its disadvantages, such as high power consumption, a complicated fabrication process regarding how to deposit ferromagnetic materials on a microactuator, and a large form factor. Orphee Cugat et al. [15] present electromagnetic microactuators using permanent magnets. Advance in material fabrication to provide thick film deposition of magnetic materials on the surface of microactuators reduces voltage and current requirements. However, thermal dissipation imposed by current CMOS technology limits the fabrication of the magnetic film on the substrate from reaching the desired characteristics.

Extensive research has been pursued in recent years to overcome these problems by incorporating other actuation methods. For example, the array of microshutters in James Webb Space Telescope (JWST) [44] opens by being pushed away, when an electromagnet runs across over the entire array of microshutters. Then, an individual side electrode latches each microshutter through the pull-in and keeps microshutters open. Each side electrode can be turned off individually to release a 
corresponding microshutter. Once a microshutter is released by its side electrode, an individual microshutter cannot be opened again without the electromagnet sweeping over the entire array.

\subsubsection{Piezoelectric Actuation}

Piezoelectric actuation utilizes the piezoelectric effect: piezoelectric material generates electric fields or potential difference across the material while it undergoes mechanical deformation; or piezoelectric material deforms in response to applied electric fields. In other words, piezoelectric actuation takes advantage of the corresponding mechanical deformation to applied electric field [23]. It has relatively lower operation voltage with low power consumption, better linearity, and fast switching time 0.1 to $1.0 \mathrm{~ms}$. For example, Young Ho Seo et al. [24] have demonstrated $3.93 \mu \mathrm{m}$ lateral displacement at $16 \mathrm{~V}$.

\subsubsection{Electrothermal Actuation}

The main advantage of electrothermal actuation is the simplicity of the fabrication method. However, in general, thermal actuation tends to have higher power consumption and slow response time. Janak Singh et al. [20] demonstrate about $10^{\circ}$ of angular displacement with approximately $10 \mathrm{~ms}$ thermal response time when their micromirror is excited at $1 \mathrm{~V}$. The out-of-plane thermal microactuator [21] takes advantages of difference in the thermal expansion coefficients of Si and Au when it experiences ohmic heating. The configuration of the thin arm and wide arm, one end of which is fixed at the substrate, has nonlinear property due to temperature dependency.

\subsubsection{Electrostatic Actuation}

Electrostatic actuation makes use of electrostatic force induced by the potential difference between a microactuator and its electrode. As its applied voltage increases, higher electrostatic force results in more displacement. For most cases, both DC bias and AC signal are used to displace a microactuator at the same time. Although the dynamics of a microactuator can be linearized within small displacement, an electrostatic microactuator is inherently nonlinear, making it more difficult for feedback control to be implemented while achieving a large displacement. Although electrostatic actuation requires higher actuation voltage than that of other actuation methods, electrostatic actuation does not require complicated fabrication methods, piezoelectric materials or ferromagnetic materials deposited on a microactuator. In addition, most electrostatic actuators require very small current, depending on the size and geometry of microactuators. In spite of this limited operation range due to the pull-in effect, nonlinear behavior in response to applied voltage, and high actuation voltage, electrostatic actuation is one of the most popular actuation methods because of its fast response time (less than $0.1 \mathrm{~ms}$ ), low power consumption, and the easiness of integration and testing with electrical control circuitry. The advantages and disadvantages of each actuation method are summarized in the following table. 
Table 2.1: Summary of Actuation Mechanism for Microactuators

\begin{tabular}{|c|c|c|}
\hline Actuation Methods & Advantages & Disadvantages \\
\hline Electromagnetic & $\begin{array}{l}\text { - Low actuation voltage } \\
\text { - Relatively large displacement }\end{array}$ & $\begin{array}{l}\text { - Difficult in fabrication of magnetic } \\
\text { material with current CMOS technology } \\
\text { - Challenge in minimizing a size of devices }\end{array}$ \\
\hline Piezoelectric & $\begin{array}{l}\text { - Higher switching speed } \\
\text { - Low power consumption }\end{array}$ & $\begin{array}{l}\text { - Small displacement range } \\
\text { - High actuation voltage }\end{array}$ \\
\hline Electrothermal & $\begin{array}{l}\text { - Easy fabrication } \\
\text { - Low actuation voltage }\end{array}$ & $\begin{array}{l}\text { - High power consumption } \\
\text { - Slow response time } \\
\text { - Thermal fatigue due to thermal cycle }\end{array}$ \\
\hline Electrostatic & $\begin{array}{l}\text { - Low power consumption } \\
\text { - Fast response time } \\
\text { - Easy to integrate and implement } \\
\text { with CMOS technology } \\
\text { - Compatible with most fabrication } \\
\text { methods }\end{array}$ & $\begin{array}{l}\text { - High actuation voltage } \\
\text { - Limited operation range due to the pull-in }\end{array}$ \\
\hline
\end{tabular}

\subsection{Conventional Electrostatic Actuation Methods}

Among many actuation methods presented in the previous section, electrostatic actuation is one of the most popular actuation methods for microactuators fabricated by MEMS technologies despite its high actuation voltage and limited operation range due to the pull-in phenomenon. The reason is that electrostatic actuators can be easily built by many fabrication methods, which are compatible with most CMOS technologies that are employed in order to manufacture modern analog and digital devices. Hence, electrostatic actuators can be packaged with control circuitry or measurement circuitry without much difficulty, allowing smaller and simpler products in various industrial applications.

\subsubsection{Voltage Control}

Among a variety of electrostatic actuation methods, voltage control is the most common method for an electrostatic actuator due to its simplicity and well-studied dynamics. For example, a parallel-plate actuator driven by voltage control is presented in Figure 2.1. As the potential difference between the top moving electrode and its bottom electrode increase, the electrostatic force also rises, pulling down the top electrode toward its bottom electrode until the mechanical restoring force exerted by the spring is equal to the electrostatic force in magnitude but opposite in direction. The governing equation of the parallel-plate actuator driven by voltage control is presented in Eq. 2.1. Using the parallel-plate actuator driven by voltage control, we study its dynamics and disadvantages originated from voltage control in this section. 


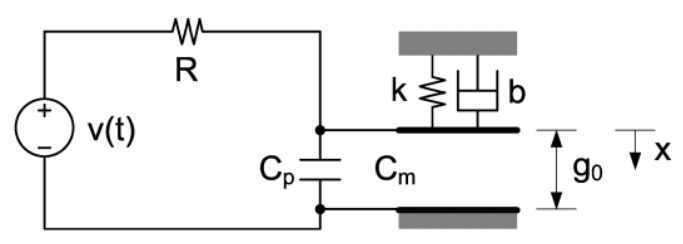

Figure 2.1 Parallel-plate electrostatic actuator driven by voltage control represented by an ideal voltage source

$$
m \ddot{X}+b \dot{X}+k X=\frac{1}{2} \varepsilon A \frac{V^{2}}{\left(g_{0}-X\right)^{2}}
$$

where $\mathbf{m}$ represents the mass of the parallel-plate actuator; $\mathbf{b}$ denotes the damping coefficient; $\mathbf{k}$ represents the spring constant; $\mathbf{X}$ denotes the displacement; $\boldsymbol{\varepsilon}$ represents the permittivity of air; $\mathbf{A}$ represents the surface area of the top electrode; $\mathbf{g}_{\mathbf{0}}$ denotes the initial gap between the top moving electrode and the fixed bottom electrode at rest; $\mathbf{V}$ denotes the potential difference between two electrodes.

Using the normalized displacement $\mathbf{x}$, which is equal to $\mathbf{X} / \mathrm{g} 0$, we rewrite Eq. 2.1 in terms of $\mathbf{x}$.

$$
m g_{0} \ddot{x}+b g_{0} \dot{x}+k g_{0} x=\frac{\varepsilon A}{2 g_{0}^{2}} \frac{V^{2}}{(1-x)^{2}}
$$

Dividing both sides with $\mathbf{g}_{0}$, we have Eq. 2.3 in terms of the normalized displacement:

$$
m \ddot{x}+b \dot{x}+k x=\frac{\varepsilon A}{2 g_{0}^{3}} \frac{V^{2}}{(1-x)^{2}}
$$

In order to determine the pull-in point and the pull-in voltage of the parallel-plate actuator, we define the net force $\mathbf{F}_{\text {net }}$ that is the sum of the mechanical restoring force $\mathbf{F}_{\mathbf{m e}}$ and the electrostatic force $\mathbf{F}_{\text {es }}$ as thus:

$$
\begin{aligned}
F_{n e t} & =F_{m e}+F_{e s} \\
& =-k x+\frac{\varepsilon A}{2 g_{0}{ }^{3}} \frac{V^{2}}{(1-x)^{2}}
\end{aligned}
$$

Since the velocity and acceleration of the parallel-plate actuator are zero at the fixed points, we determine the fixed point $\mathbf{x}^{*}$ of the parallel-plate actuator by setting $\mathbf{F}_{\text {net }}$ to be zero. To evaluate the stability of the fixed point $\mathbf{x}^{*}$, we need to determine the derivative of $\mathbf{F}_{\text {net }}$ with respect to $\mathbf{x}$ at the fixed point $\mathbf{x}^{*}$ as thus:

$$
\begin{aligned}
& \text { if }\left.\frac{\partial F_{n e t}}{\partial x}\right|_{x=x^{*}}<0, \text { then } x^{*} \text { is stable; } \\
& \text { if }\left.\frac{\partial F_{n e t}}{\partial x}\right|_{x=x^{*}}>0, \text { then } x^{*} \text { is unstable; } \\
& \text { if }\left.\frac{\partial F_{n e t}}{\partial x}\right|_{x=x^{*}}=0, \text { then } x^{*} \text { is a saddle node. }
\end{aligned}
$$


Hence, the derivative of $\mathbf{F}_{\text {net }}$ with respect to $\mathbf{x}$ is equal to Eq. 2.5:

$$
\frac{\partial F_{n e t}}{\partial x}=-k+\frac{\varepsilon A}{g_{0}{ }^{3}} \frac{V^{2}}{(1-x)^{3}}
$$

Setting Eq. 2.4 and Eq. 2.5 to zero, we determine the pull-in point $\mathbf{x}_{\mathbf{p i}}$ and the pull-in voltage $\mathbf{V}_{\mathbf{p i}}$ in Eq. 2.6, which are identical to the results presented by J.I. Seeger and B.E. Boser [7]:

$$
x_{p i}=\frac{1}{3}, V_{p i}=\sqrt{\frac{8 \mathrm{~kg}_{0}^{3}}{27 \varepsilon A}}
$$

With respect to the pull-in voltage derived in Eq. 2.6, we normalize the input voltage and transform the governing equation Eq. 2.3 into the non-dimensional equation as thus:

$$
\begin{aligned}
\ddot{x}+2 \xi \omega_{m} \dot{x}+\omega_{m}^{2} x & =\frac{\varepsilon A}{2 m g_{0}{ }^{3}} \frac{V^{2}}{(1-x)^{2}} \\
& =\frac{4 \omega_{m}{ }^{2}}{27} \frac{V_{n}{ }^{2}}{(1-x)^{2}}
\end{aligned}
$$

where $\xi$ represents the damping ratio; $\boldsymbol{\omega}_{\mathrm{m}}$ denotes the mechanical natural frequency of the parallel plate actuator; and $\mathbf{V}_{\mathbf{n}}$ represents the normalized input voltage.

Using the normalized governing equation Eq. 2.7, we determine the fixed points of the parallel-plate actuator driven by voltage control and their stability by solving Eq. 2.8 and Eq. 2.9, which is the first derivative of $\mathbf{F}_{\text {net }}$ in terms of the normalized displacement $\mathbf{x}$. The fixed points of the parallel-plate actuator driven by conventional voltage control are presented in Figure 2.2, where the solid line represents the stable fixed points and the dotted line denotes the unstable fixed points. As shown in Eq. 2.6, the parallel-plate actuator undergoes the saddle-node bifurcation at $1 / 3$ of its original gap.

$$
\begin{aligned}
F_{n e t} & =F_{m e}+F_{e s} \\
& =-\omega_{m}{ }^{2} x+\frac{4 \omega_{m}{ }^{2}}{27} \frac{V_{n}{ }^{2}}{(1-x)^{2}} \\
& =\omega_{m}{ }^{2}\left(-x+\frac{4}{27} \frac{V_{n}{ }^{2}}{(1-x)^{2}}\right) \\
\frac{\partial F_{n e t}}{\partial x} & =-\omega_{m}{ }^{2}+\frac{8 \omega_{m}{ }^{2}}{27} \frac{V_{n}{ }^{2}}{(1-x)^{3}} \\
& =\omega_{m}{ }^{2}\left(-1+\frac{8}{27} \frac{V_{n}{ }^{2}}{(1-x)^{3}}\right)
\end{aligned}
$$




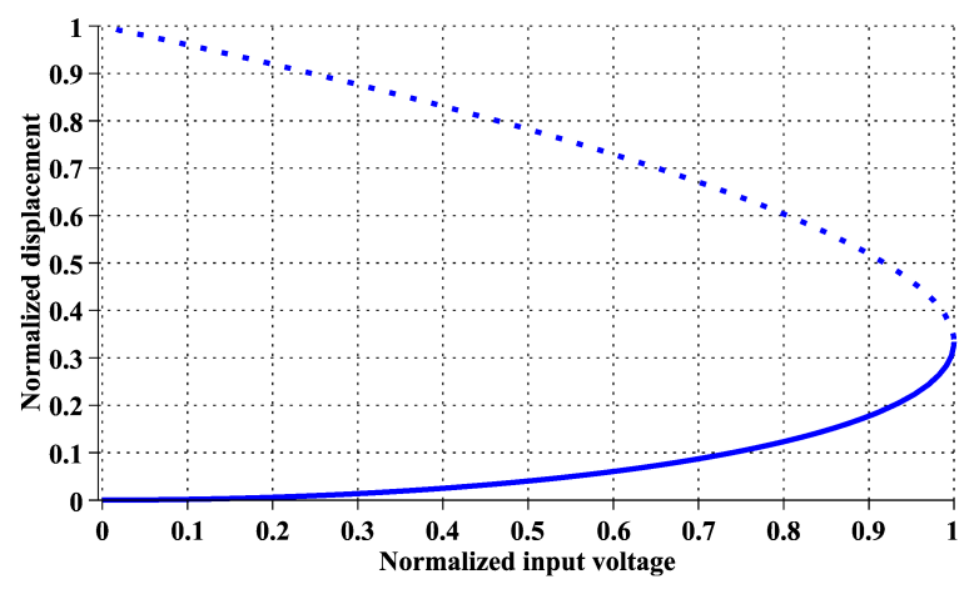

Figure 2.2 Fixed points of the parallel-plate actuator driven by voltage control: the solid blue line represents the stable fixed points; the dotted blue line denotes the unstable fixed points; and the saddle-node bifurcation occurs at $1 / 3$ of the original gap at rest.

We also create the numerical model of the parallel-plate actuator driven by voltage control described by Eq. 2.1 in Matlab/Simulink. The physical properties of the parallel-plate actuator used in its numerical model are presented as thus:

Table 2.2 Physical parameters of the parallel-plate actuator

\begin{tabular}{ccc}
\hline \hline Parameter & Symbol & Value \\
\hline \hline Spring constant & $\mathrm{k}$ & $1[\mathrm{mN} / \mathrm{m}]$ \\
Mass & $\mathrm{m}$ & $2 \mathrm{e}-10[\mathrm{~kg}]$ \\
Area & $\mathrm{A}$ & $10000\left[\mu \mathrm{m}^{2}\right]$ \\
Initial gap & $\mathrm{g}_{0}$ & $50[\mu \mathrm{m}]$ \\
Natural frequency of Actuator & $\mathrm{f}_{0}$ & $356[\mathrm{~Hz}]$ \\
Damping coefficient & $\mathrm{b}$ & $0.8944[\mu \mathrm{N} \cdot \mathrm{s} / \mathrm{m}]$ \\
Damping ratio & $\xi$ & 1 \\
Resistance & $\mathrm{R}$ & $40[\Omega]$ \\
Permittivity of air & $\varepsilon$ & $8.854 \mathrm{e}-12[\mathrm{~F} / \mathrm{m}]$ \\
Initial capacitance & $\mathrm{C}_{0}$ & $1.77[\mathrm{fF}]$ \\
Parasitic capacitance & $\mathrm{C}_{\mathrm{p}}$ & $1.77[\mathrm{pF}]$ \\
Pull-in point & $\mathrm{X}_{\mathrm{pi}}$ & $16.7[\mu \mathrm{m}]$ \\
Pull-in voltage & $\mathrm{V}_{\mathrm{pi}}$ & $20.45[\mathrm{~V}]$ \\
\hline \hline
\end{tabular}




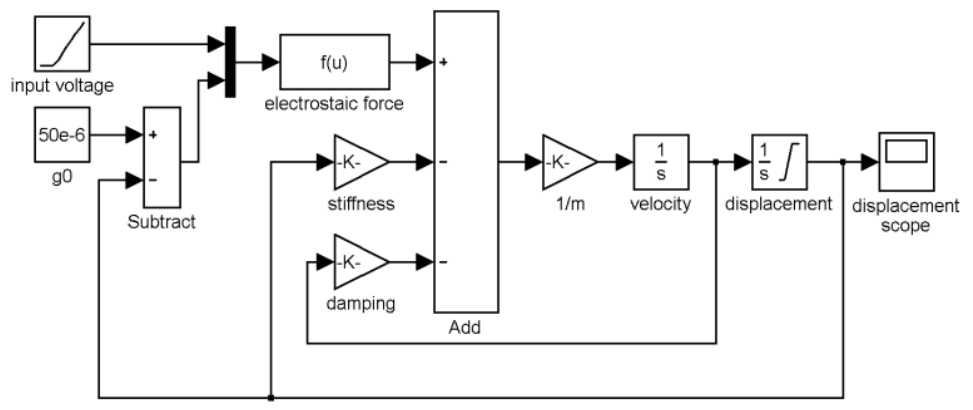

Figure 2.3 Numerical model of the parallel-plate actuator driven by voltage control described by the governing equation of Eq. 2.1.

The displacement of the parallel-plate actuator is presented in Figure 2.4, as the actuation voltage increases. As presented in Figure 2.4, the numerical results are in good agreement with the analytical results, and the disadvantages of voltage control are clear: a limited operation range caused by the pull-in phenomenon and a high actuation voltage required to drive the parallel-plate actuator.

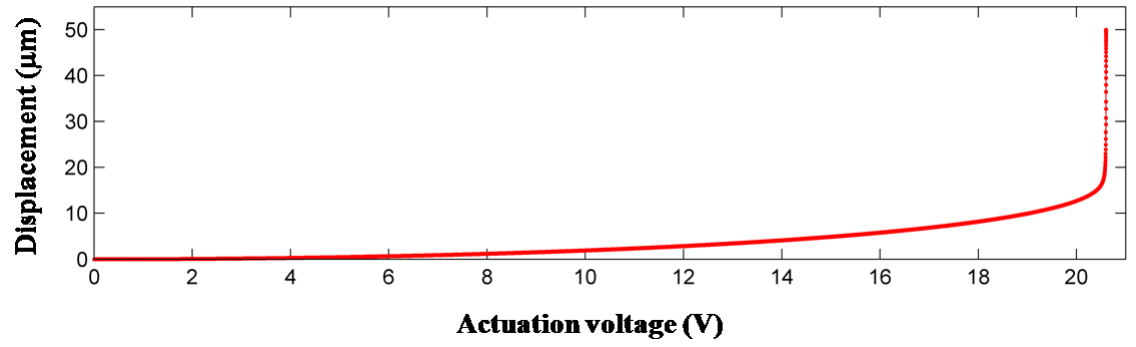

Figure 2.4 Displacement of the parallel-plate actuator driven by voltage control: the pull-in voltage is $20.45 \mathrm{~V}$; and the pull-in point is $16.7 \mu \mathrm{m}$, which is equal to $1 / 3$ of the original gap, $50 \mu \mathrm{m}$.

\subsubsection{Charge Control}

Instead of controlling the voltage applied to a microactuator, one can control the charge stored in a microactuator by using a current source. The simplified schematic of the parallel-plate actuator driven by charge control is presented in Figure 2.5. Since the electric field is conservative, the electrostatic force applied on the top moving electrode of the parallel-plate actuator is equal to the partial derivative of the potential energy, presented in Eq. 2.10, stored in the electric fields inside the parallel-plate actuator and its parasitic capacitance in terms of the displacement as shown in Eq. 2.11:

$$
U_{e s}=\frac{1}{2} \frac{Q^{2}}{C}=\frac{1}{2} \frac{Q^{2}}{\left(C_{p}+C_{m}\right)}=\frac{1}{2} \frac{Q^{2}}{\left(C_{p}+\frac{\varepsilon A}{g_{0}-X}\right)}=\frac{1}{2} \frac{\left(g_{0}-X\right) Q^{2}}{\left(C_{p}\left(g_{0}-X\right)+\varepsilon A\right)}
$$

where $\mathbf{U}_{\text {es }}$ represent the potential energy stored in both the parallel-plate actuator and its parasitic capacitance $\mathbf{C}_{\mathbf{p}} ; \mathbf{C}$ denotes the sum of the capacitance of the parallel-plate actuator $\mathbf{C}_{\mathbf{m}}$ and its 
parasitic capacitance $\mathbf{C}_{\mathbf{p}} ; \mathbf{g}_{\mathbf{0}}$ represents the initial gap; and $\mathbf{X}$ denotes the displacement of the parallelplate actuator.

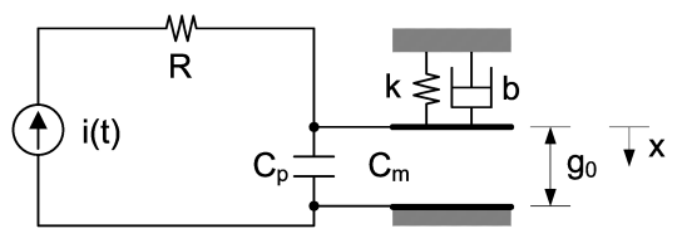

Figure 2.5 Parallel-plate actuator driven by charge control; a charge source is represented by an ideal current source.

$$
\frac{\partial U_{e s}}{\partial X}=F_{e s}=\frac{1}{2} \frac{\varepsilon A Q^{2}}{\left(C_{p}\left(g_{0}-X\right)+\varepsilon A\right)^{2}}
$$

Using the normalized displacement $\mathbf{x}$ and the ratio $\mathbf{r}$ of $\mathbf{C}_{\mathbf{p}}$ to $\mathbf{C}_{\mathbf{0}}$, Eq. 2.11 is further simplified as thus:

$$
F_{e s}=\frac{1}{2 \varepsilon A g_{0}} \frac{Q^{2}}{(1+r(1-x))^{2}}
$$

Hence, the governing equation of the parallel-plate actuator driven by charge control is as thus:

$$
\begin{aligned}
\ddot{x}+2 \xi \omega_{m} \dot{x}+\omega_{m}{ }^{2} x & =\frac{1}{2 \varepsilon A g_{0}} \frac{Q^{2}}{(1+r(1-x))^{2}} \\
& =\frac{4}{27} \omega_{m}{ }^{2} \frac{(r+1)^{3} Q_{n}{ }^{2}}{r(1+r(1-x))^{2}}
\end{aligned}
$$

where $\mathbf{x}$ represents the normalized displacement of the parallel-plate actuator; $\boldsymbol{\xi}$ denotes the damping ratio; $\boldsymbol{\omega}_{\mathrm{m}}$ represents the mechanical natural frequency of the parallel plate actuator; $\mathbf{Q}$ denotes the total charge stored in the parallel-plate actuator and its parasitic capacitance $\mathbf{C}_{\mathbf{p}} ; \mathbf{r}$ represents the ratio of the parasitic capacitance $\mathbf{C}_{\mathbf{p}}$ to the initial capacitance of the parallel-plate actuator $\mathbf{C}_{\mathbf{0}}$ at rest; and $\mathbf{Q}_{\mathbf{n}}$ denotes the normalized input charge.

As presented in Eq. 2.13, the parallel-plate actuator with $\mathbf{C}_{\mathbf{p}}$ smaller than one half of $\mathbf{C}_{\mathbf{0}}$ behaves as a linear system, because the nonlinear effect of the electrostatic force is minimized. Hence, the parallelplate actuator does not experience the pull-in phenomenon when it is actuated by charge control. The normalized displacement of the parallel-plate actuator driven by charge control in terms of various parasitic capacitances is presented in Figure 2.6. However, in reality, the parasitic capacitance $\mathbf{C}_{\mathbf{p}}$ is much higher than the capacitance $\mathbf{C}_{\mathbf{0}}$ of an electrostatic actuator itself, depending on the geometry, and configuration of an electrostatic microactuator. This high parasitic capacitance acts as a voltage source providing enough charge to cause the pull-in [7]. In order to determine where the pull-in occurs under various parasitic capacitances, we evaluate Eq. 2.13 while varying the ratio of $\mathbf{C}_{\mathbf{p}}$ to $\mathbf{C}_{\mathbf{0}}$. The pull-in points of the parallel-plate actuator are presented in Figure 2.7, when the charge control method is implemented to drive the parallel-plate actuator with various parasitic capacitances. As presented in Figure 2.7, the charge control method is able to extend the operation range of the parallel-plate actuator up to $40 \%$ of the initial gap when the parasitic capacitance is 5 times bigger 
than the initial capacitance $\mathbf{C}_{\mathbf{0}}$ of the parallel-plate actuator, but fails to stabilize the parallel-plate actuator when the parasitic capacitance is much higher than the initial capacitance $\mathbf{C}_{\mathbf{0}}$.

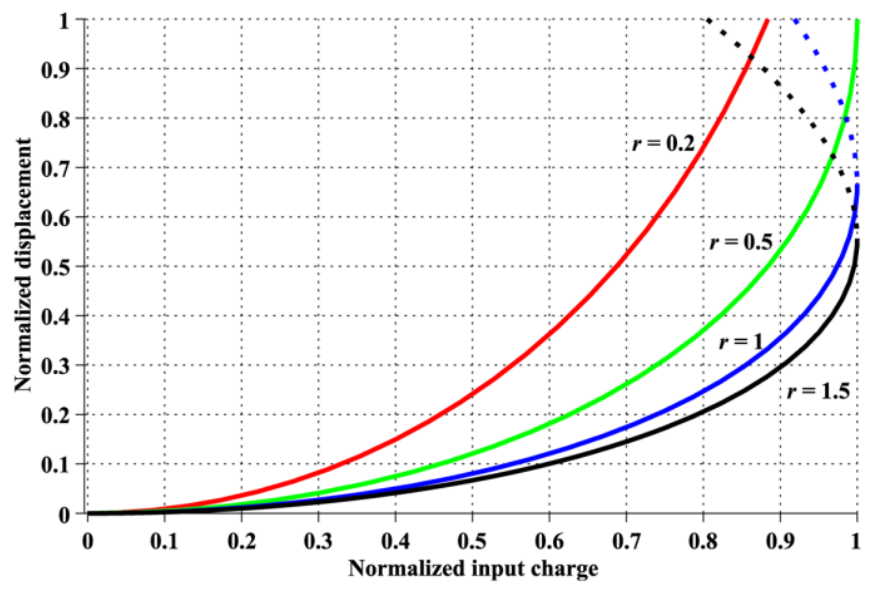

Figure 2.6 Fixed points of the parallel-plate actuator driven by charge control with various parasitic capacitances: $\mathbf{r}$ represents the ratio of the parasitic capacitance $\mathbf{C}_{\mathbf{p}}$ and the initial capacitance of the parallel-plate actuator $\mathbf{C}_{\mathbf{0}}$ at rest.

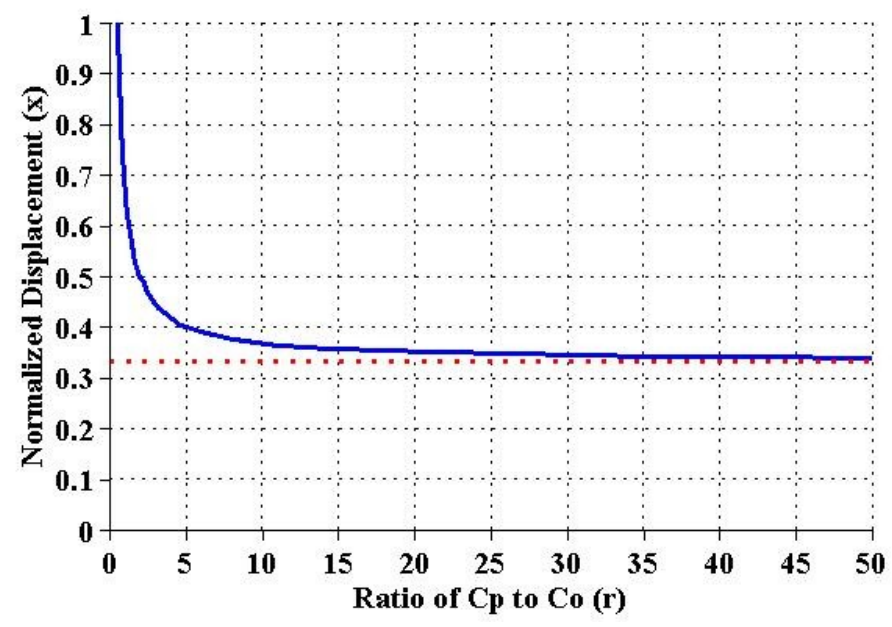

Figure 2.7 Saddle-node bifurcation points of the parallel-plate actuator driven by voltage control and charge control: the red dotted line represents the pull-in points of the parallel-plate actuator driven by voltage control; and the blue solid line denotes the pull-in points of the parallel-plate actuator driven by charge control with various parasitic capacitances.

Moreover, we create the numerical model of the parallel-plate actuator driven by charge control in Matlab/Simulink, which is described by Eq. 2.13. The physical properties of the parallel-plate actuator used in its numerical model are same as those in the previous numerical model. The numerical model created in Matlab/Simulink is presented in Figure 2.8, and the displacement of the 
parallel-plate actuator driven by charge control is shown in Figure 2.9, as more charges are injected into the parallel-plate actuator and its parasitic capacitance. As presented in Figure 2.9, the parallelplate actuator driven by charge control also undergoes the charge pull-in [7].

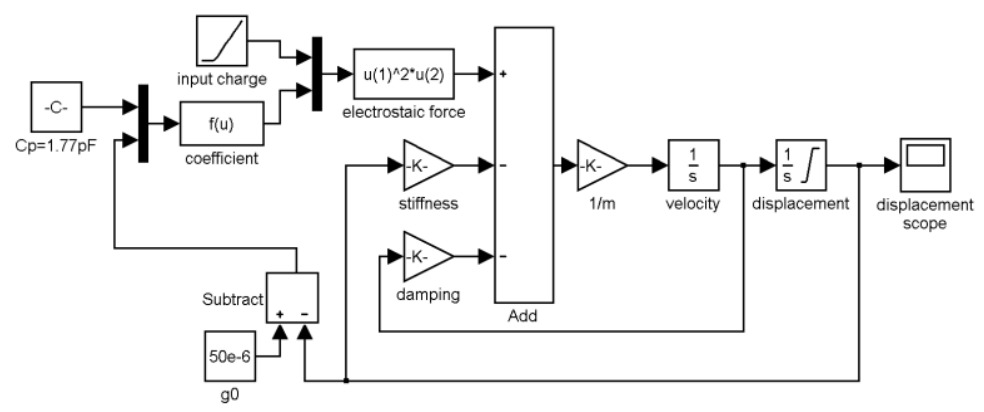

Figure 2.8 Numerical model of the parallel-plate electrostatic actuator driven by charge control, which is created in Matlab/Simulink.

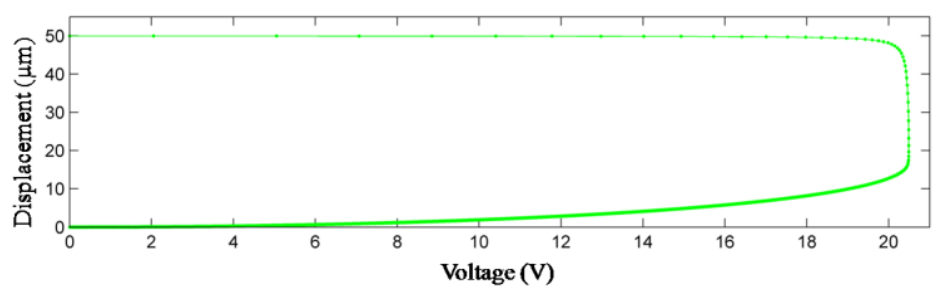

Figure 2.9 Displacement of the parallel-plate actuator driven by charge control as charge is accumulated in both the parallel-plate actuator and its parasitic capacitance.

The current source presented in Figure 2.5 can be implemented in many different ways. For example, a transimpedance amplifier with a switched capacitor input [7, 45], a photovoltaic source and an optocoupler [46] have been used to drive an electrostatic actuator as a charge source.

\subsubsection{Voltage Control with a Feedback Capacitor}

Although charge control can stabilize an electrostatic actuator with very small parasitic capacitance, it is more difficult to design and build a charge source that can inject very small predetermined current over a wide range of voltages. Hence, E.K. Chan and R.W. Dutton [9] and D.H.S. Maithripala et al. [10] develop the voltage control method with a feedback capacitor in series to extend the operation range of an electrostatic actuator. The schematic of the parallel-plate actuator driven by voltage control with a feedback capacitor in series is presented in Figure 2.10. This voltage control method can extend the operation range of an electrostatic actuator in the presence of small parasitic capacitance, while using much higher actuation voltage than that of conventional voltage control. However, it turns out that this voltage control method also suffers from the pull-in in the presence of high parasitic capacitance. 


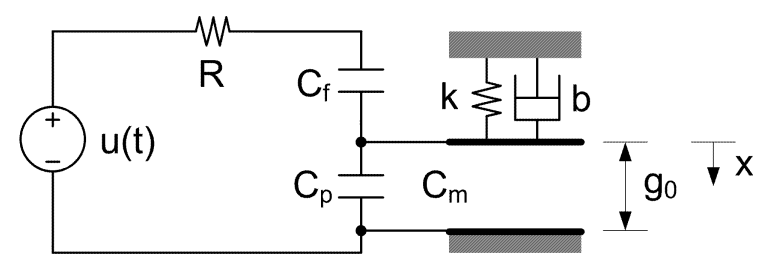

Figure 2.10 Schematic of the parallel-plate actuator driven by voltage control with a feedback capacitor $\mathbf{C}_{\mathbf{f}}$ connected in series with a parallel-plate actuator and its parasitic capacitance.

In order to derive the governing equation of the parallel-plate actuator driven by voltage control with a feedback capacitor in series, we first determine the voltage applied on the parallel-plate actuator as thus:

$$
V=\frac{Z_{2}}{Z_{1}+Z_{2}} u=\frac{\frac{1}{s\left(C_{p}+C_{m}\right)}}{\frac{1}{s C_{f}}+\frac{1}{s\left(C_{p}+C_{m}\right)}} u=\frac{1}{\frac{\left(C_{p}+C_{m}\right)}{C_{f}}+1} u=\frac{C_{f}}{\left(C_{p}+C_{m}\right)+C_{f}} u
$$

where $\mathbf{V}$ represents the voltage applied on the parallel-plate actuator, which is determined by the capacitive voltage divider presented in Figure 2.10; $\mathbf{u}$ denotes the input voltage ; $\mathbf{Z}_{1}$ represents the impedance of the feedback capacitor; and $\mathbf{Z}_{2}$ denotes the impedance of the parallel-plate actuator and its parasitic capacitance.

Substituting Eq. 2.14 into Eq. 2.3, we derive the governing equation of Eq. 2.15:

$$
\ddot{x}+2 \xi \omega_{m} \dot{x}+\omega_{m}^{2} x=\frac{\varepsilon A}{2 m g_{0}{ }^{3}} \frac{u^{2}}{(1-x)^{2}}\left(\frac{C_{f}}{\left(C_{p}+C_{m}\right)+C_{f}}\right)^{2}
$$

To simplify the governing equation, we describe the voltage change in terms of the normalized displacement of the parallel-plate actuator and define the ratio of various capacitances as thus:

$$
C_{m}=\frac{\varepsilon A}{g_{0}} \frac{1}{(1-x)}=\frac{C_{0}}{(1-x)}, r=\frac{C_{p}}{C_{0}}, r_{2}=\frac{C_{f}}{C_{0}}
$$

Substituting Eq. 2.16 into Eq. 2.15, we derive the governing equation in terms of the normalized displacement and the input voltage as thus:

$$
\begin{aligned}
\ddot{x}+2 \xi \omega_{m} \dot{x}+\omega_{m}{ }^{2} x & =\frac{\varepsilon A}{2 m g_{0}{ }^{3}} \frac{u^{2}}{(1-x)^{2}}\left(\frac{\frac{C_{f}}{C_{0}}}{\left(\frac{C_{p}}{C_{0}}+\frac{1}{1-x}\right)+\frac{C_{f}}{C_{0}}}\right)^{2} \\
& =\frac{\varepsilon A}{2 m g_{0}{ }^{3}} \frac{u^{2}}{(1-x)^{2}}\left(\frac{r_{2}}{\left(r+\frac{1}{1-x}\right)+r_{2}}\right)^{2} \\
& =\frac{\varepsilon A}{2 m g_{0}{ }^{3}} \frac{r_{2}{ }^{2} u^{2}}{\left(\left(r+r_{2}\right)(1-x)+1\right)^{2}}
\end{aligned}
$$


Using the pull-in voltage of the parallel plate actuator defined in Eq. 2.6, we further simply Eq. 2.17 into Eq. 2.18:

$$
\ddot{x}+2 \xi \omega_{m} \dot{x}+\omega_{m}^{2} x=\frac{4}{27} \frac{\omega_{m}^{2} r_{2}^{2} u_{n}^{2}}{\left(\left(r+r_{2}\right)(1-x)+1\right)^{2}}
$$

Using the normalized governing equation of Eq. 2.18, we determine the fixed points of the parallelplate actuator driven by voltage control with a feedback capacitor in series and their stability, presented in Figure 2.11, by solving Eq. 2.19.

$$
\begin{aligned}
F_{n e t} & =F_{m e}+F_{e s} \\
& =-\omega_{m}^{2} x+\frac{4}{27} \frac{\omega_{m}^{2} r_{2}{ }^{2} u_{n}{ }^{2}}{\left(\left(r+r_{2}\right)(1-x)+1\right)^{2}} \\
& =\omega_{m}^{2}\left(-x+\frac{4}{27} \frac{r_{2}{ }^{2} u_{n}^{2}}{\left(\left(r+r_{2}\right)(1-x)+1\right)^{2}}\right)
\end{aligned}
$$

As mentioned in the previous section, the voltage control with a feedback capacitor in series can extend the operation range of an electrostatic actuator in the absence of high parasitic capacitance. However, the operation range of the parallel-plate actuator is limited by the parasitic capacitance of the parallel-plate actuator as presented in Figure 2.11.

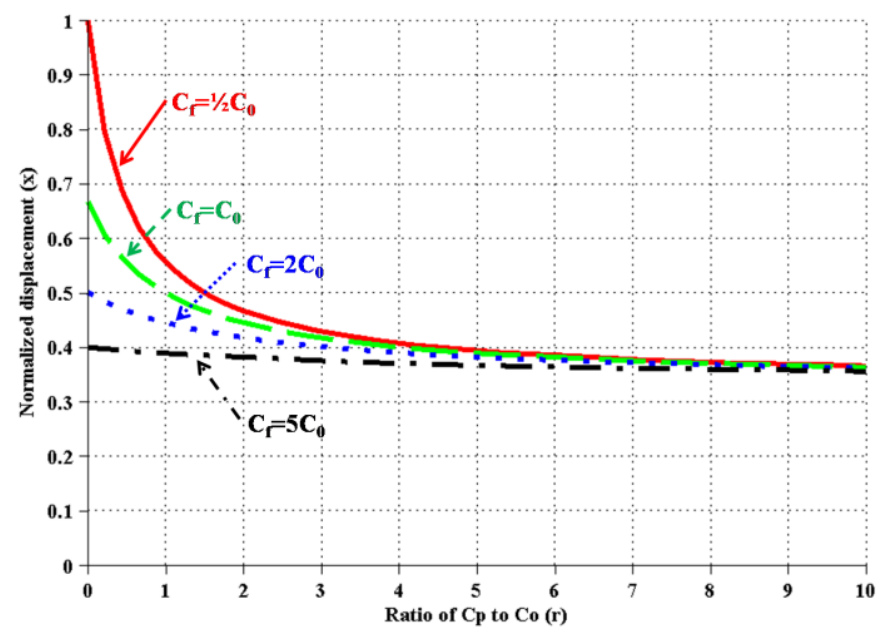

Figure 2.11 Pull-in points of the parallel-plate actuator driven by voltage control with various sizes of feedback capacitors in series over a wide range of the parasitic capacitance.

\subsection{Electrostatic Actuation through Impedance Transformation}

Unlike all actuation methods described in the previous sections, this electrostatic actuation method through impedance transformation requires a high frequency $\mathrm{AC}$ input signal even for a static operation and a modulated $\mathrm{AC}$ input signal for a dynamic operation to take full advantage of impedance change in an electrostatic actuator over a range of different frequencies. Moreover, a 
resonant circuit has been widely used in various industrial applications as a sensing element: small change in the physical properties of interest causes deviation in the resonance frequency of the resonant circuit or impedance change. Through the measurement of these variations, the physical properties of interest have been monitored.

\subsubsection{Signal Transformer}

A signal transformer or a transformer in general has been widely used in various industrial applications due to its unique properties, such as voltage or current conversion and galvanic isolation between two adjacent circuits. It has been used for wideband impedance transformation in RF applications as well, providing high $\mathrm{AC}$ output voltage from low AC input voltage over a wide range of frequencies (up to $1 \mathrm{MHz}$ ) or vice versa.

H. Camon et al. [25] study how to drive an electrostatic actuator with a signal transformer. A signal transformer is excited by low voltage AC signal, generating a high voltage AC signal, which is rectified into a high voltage DC signal by a full-bridge rectifier. Then, a high voltage rectified signal is used to drive an electrostatic actuator. Because a voltage gain of a signal transformer depends a winding ratio of the primary and secondary side of a transformer, a voltage gain does not vary, regardless of the frequency of a low voltage AC signal. Hence, a signal transformer is well-suited to drive an electrostatic actuator at its mechanical resonance.

\subsubsection{Resonant Circuit}

$\mathrm{L}, \mathrm{T}$, and $\pi$ networks implemented with discrete components of resistors, capacitors, and inductors have been used for narrowband impedance transformation in many RF applications. Unlike a signal transformer, these networks only work at a specific frequency or over very narrow range of frequencies. Hence, they are called the narrowband impedance transformation networks.

At the resonant frequency of these circuits, a voltage gain is highest in a series resonant circuit, whereas a current gain is greatest in a parallel resonant circuit. Because we intend to amplify low input voltage into high actuation voltage, the series resonant circuits are exclusively used in this thesis. The frequency responses of the series resonant circuits are presented in Figure 2.12. As an electrostatic actuator displaces toward its bottom electrode, its capacitance increases and the resonant frequency of the resonant drive circuits decreases in turn. Because the frequency of low voltage AC signal is fixed at the initial resonant frequency of the resonant drive circuits, its voltage gain drops, reducing the actuation voltage through this inherent negative feedback. When this inherent negative feedback is large enough, the resonant drive circuits are able to displace an electrostatic actuator over its entire gap without the saddle-node bifurcation. In other words, if an electrostatic actuator has small parasitic capacitance compared to the quality factor of the resonant drive circuits, the resonant drive circuits are capable of driving an electrostatic actuator beyond the pull-in point. J. M. Kyynäräinen et al. [26] present their research of driving an electrostatic actuator through an $\mathrm{L}$ impedance transformation network that generates high actuation voltage from low AC input voltage through passive amplification at the resonance of the LC circuit. B. Cagdaser et al. [27] also use an LC tank circuit to drive an electrostatic actuator with low voltage AC signal at its electrical resonance. They However, their research does not provide any analytical model of an electrostatic actuator driven by 
their LC circuits and any insight on relationship between its parasitic capacitance and operation range. Hence, in this paper, we present the analytical and numerical model of the parallel-plate actuator driven by the resonant drive circuit to facilitate its study of nonlinear dynamics and to further understand how the electrostatic actuator's parasitic capacitance is intertwined with its operation range and the quality factor of the resonant drive circuit.

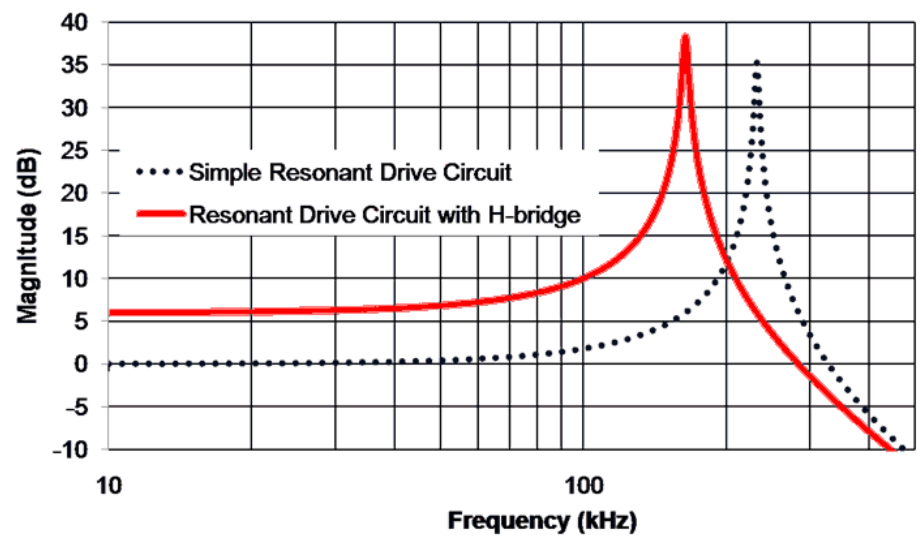

Figure 2.12 Frequency responses of two resonant drive circuits: an unbalanced resonant drive circuit depicted by the dotted line and a balanced resonant drive circuit presented in the red solid line.

\subsection{Summary}

A high actuation voltage has been one of the challenges for the electrostatically actuated micromirror or torsional actuator. Moreover the operation range of the micromirror is limited to $44 \%$ of the maximum angular displacement due to the saddle-node bifurcation, and the angular displacement measurement of the micromirror is difficult to implement due to its minute size and constraints imposed by its fabrication methods. To overcome these problems, we present the resonant drive circuit that operates at a much lower supply voltage, provide the complete analytical models of the micromirror coupled with the resonant drive circuit, and we present the new method of the angular displacement measurement of the micromirror. The presented analytical models confirm that the resonant drive circuit enables us to actuate the micromirror at low supply voltage and that its operation range can be extended in the presence of low parasitic capacitance. Furthermore, unlike conventional high voltage actuation circuits, the resonant drive circuit does not pose any industrial hazard related to high voltage because a high voltage power supply is not required for operation. Hence, it is inherently robust and can be safely implemented in many industrial and medical applications where high voltage is not allowed. Finally, the new method of the angular displacement measurement of the micromirror allows feedback control to be easily implemented without any modification on the micromirror itself. 


\section{Chapter 3 \\ Parallel-Plate Actuator Driven by Resonant Drive Circuit}

In this chapter, we derive an analytical model of the parallel-plate actuator coupled with resonant drive circuits in order to analyze its nonlinear behavior and provide a complete theoretical understanding of its nonlinear dynamics. We create its numerical model to validate its analytical results as well. Using this analytical model and numerical model, we emphasize more qualitative properties of the coupled physics of the parallel-plate actuator driven by the resonant drive circuit than its quantitative details. In other words, we are more interested in a global behavior of the parallel-plate actuator driven by the resonant drive circuits than its localized solutions, which have been presented by other researchers through the small signal analysis [26, 27]. Moreover, the analytical model of the parallel-plate actuator driven by the resonant drive circuit gives insight into an intricate process of how high parasitic capacitance of the parallel-plate actuator interacts with the quality factor of the resonant drive circuit and affects the inherent negative feedback of the resonant drive circuit, resulting in less robustness to the saddle-node bifurcation.

This chapter is organized as follows. In Section 3.1, an analytical model of the parallel-plate actuator coupled with the resonant drive circuit controlled by the amplitude modulation scheme is derived through the multiple scale methods [43], which allow us to convert a linear time-varying system of the resonant drive circuit into a linear time-invariant system, which is coupled into a nonlinear system of the parallel-plate actuator. Then, the governing equation of the resonant drive circuit is transformed into an algebraic equation through a frequency response in terms of the initial quality factor of the resonant drive circuit and the parasitic capacitance of the parallel-plate actuator. In Section 3.2, the fixed points of the parallel-plate actuator driven by the resonant drive circuit are evaluated, and their stability is also analyzed and presented. In Section 3.3, an analytical model of the parallel-plate actuator driven by the resonant drive circuit, which is controlled by the frequency modulation, is derived and the stability of its fixed points are evaluated in Section 3.4. In Section 3.5, a new displacement measurement method is discussed in general when the parallel-plate actuator is driven by the resonant drive circuit. In Section 3.6, a numerical model of the parallel-plate actuator driven by the resonant drive circuit is built to validate its analytical model presented in Section 3.1 and Section 3.2. Moreover, the numerical model of the phase detector introduced in Section 3.5 is created to measure the displacement of the parallel-plate actuator as well. In Section 3.7, the experiment results obtained from the parallel plates are presented and compared with the analytical and numerical results.

\subsection{Analytical Model of Parallel-Plate Actuator Coupled with Resonant Drive Circuit Controlled by Amplitude Modulation}

In this section, we derive and present the analytical model of the parallel-plate electrostatic actuator to analyze its nonlinear dynamics when it is actuated by the resonant drive circuit controlled by the amplitude modulation scheme as presented in Figure 3.1. Moreover, the parasitic capacitance of the parallel-plate actuator is included in this analytical model so that its effect on the stability of the parallel-plate actuator is thoroughly studied and analyzed. 


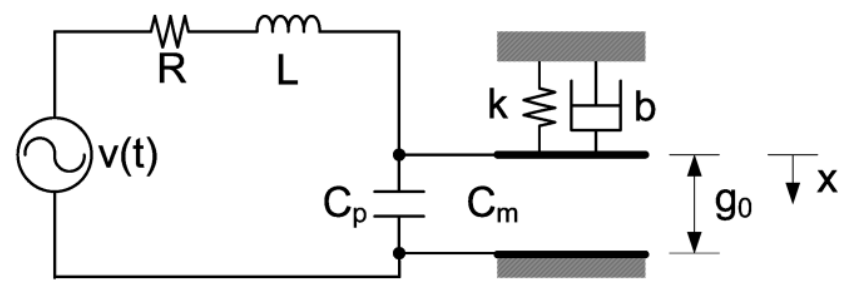

Figure 3.1 Schematic of the parallel-plate electrostatic actuator coupled with the resonant drive circuit driven by an AC voltage source without a DC bias

Since an electrostatic actuator can be modeled as a variable capacitor, the parallel-plate actuator and the resonant drive circuit are coupled through the displacement or the capacitance of the parallel-plate actuator represented by $\mathbf{C}_{\mathbf{m}}$ and its parasitic capacitance $\mathbf{C}_{\mathbf{p}}$. Their governing equations are shown thus:

$$
\begin{gathered}
m \ddot{X}+b \dot{X}+k X=\frac{1}{2} \varepsilon A \frac{V^{2}}{\left(g_{0}-X\right)^{2}} \\
L \ddot{q}+R \dot{q}+\frac{1}{C_{p}+C_{m}} q=u \\
q=\left(C_{p}+C_{m}\right) V
\end{gathered}
$$

where $\mathbf{m}$ represents the mass of the parallel-plate actuator; $\mathbf{b}$ denotes the damping coefficient; $\mathbf{k}$ represents the spring constant; $\mathbf{X}$ denotes the displacement; $\mathbf{C}_{\mathbf{m}}$ represents the capacitance of the parallel-plate actuator; $\boldsymbol{\varepsilon}$ represents the permittivity of air; $\mathbf{A}$ represents the surface area; $\mathbf{g}_{\mathbf{0}}$ denotes the initial gap between the top moving electrode and the fixed bottom electrode at rest; $\mathbf{V}$ denotes the potential difference between two electrodes; $\mathbf{L}$ denotes the inductance of the inductor; $\mathbf{q}$ represents total charge stored in both the parallel-plate actuator $\mathbf{C}_{\mathbf{m}}$ and its parasitic capacitor $\mathbf{C}_{\mathbf{p}}$ at any given time; $\mathbf{R}$ represents losses of an inductor and parasitic resistance in the resonant drive circuit, such as ESR (Equivalent Series Resistance); u represents the peak voltage of an input AC voltage source.

In reality, the inductance $\mathbf{L}$ and the resistance $\mathbf{R}$ in Eq. 3.2 vary over the frequency and are intertwined: an inductor with higher inductance tends to have more parasitic resistance; a ferrite core or iron core of an inductor causes a variety of magnetic losses, such as hysteresis loss and eddy current loss, respectively. An inductor with an air core does not suffer from these losses, but, its size is much bigger than the size of a ferrite core inductor that has the same inductance. Moreover, when the current through an inductor increases more than the rated saturation current of an inductor, its inductance drops rapidly and renders Eq. 3.2 invalid. Hence, the caution must be addressed. In this resonant drive circuit, the frequency of the input $\mathrm{AC}$ voltage remains constant, and the current through an inductor is kept below the saturation current of an inductor over entire analysis and simulation to avoid these nonlinearities. Hence, as long as these conditions are met, an inductor can be considered as a linear time-invariant component, and Eq. 3.2 is valid.

After the normalization of the displacement using the following relationship, $\boldsymbol{X}=\boldsymbol{g}_{\mathbf{0}} \boldsymbol{x}$, Eq. 3.1 is converted into Eq. 3.4: 


$$
m \ddot{x}+b \dot{x}+k x=\frac{1}{2} \frac{\varepsilon A}{g_{0}^{3}} \frac{V^{2}}{(1-x)^{2}}
$$

Furthermore, Eq. 3.2 representing the resonant drive circuit is linear with a time-varying parameter $\mathbf{C}_{\mathbf{m}}$, which varies slowly with respect to the input $\mathrm{AC}$ voltage. In other words, a very large number of cycles of the input signal elapse by the time there is any significant change in the displacement or the capacitance of the parallel-plate actuator. Simply speaking, we can assume that $\mathbf{C}_{\mathbf{m}}$ is a constant on a fast time scale. Hence, we treat the resonant drive circuit as a linear time-invariant (LTI) system with respect to a fast time scale. However, we regard $\mathbf{C}_{\mathbf{m}}$ as a variable on a slow time scale, because $\mathbf{C}_{\mathbf{m}}$ depends on the displacement, which is, in turn, a function of a slow time scale without loss of accuracy. Thus, the resonant drive circuit depicted by Eq. 3.2 is treated as a LTI system and its frequency response is derived from the Laplace transformation of Eq. 3.2, resulting in Eq. 3.5 and Eq. 3.6. Through the frequency response presented in Eq. 3.6, we derive the voltage gain of the resonant drive circuit, which depends on the capacitance of the parallel-plate actuator. Because the resonant drive circuit amplifies the input AC voltage at its electrical resonance, the actuation voltage applied on the parallel-plate actuator is expressed, in general, in terms of voltage gain that depends on the displacement or the capacitance of the parallel-plate actuator $\mathbf{C}_{\mathbf{m}}$.

$$
\begin{gathered}
H(s)=\frac{V(s)}{U(s)}=\frac{1}{L C s^{2}+R C s+1}=\frac{1}{L\left(C_{p}+C_{m}\right) s^{2}+R\left(C_{p}+C_{m}\right) s+1} \\
H(j \omega)=\frac{V(j \omega)}{U(j \omega)}=\frac{1}{1-L C \omega^{2}+j \omega R C}=\frac{1}{1-L\left(C_{p}+C_{m}\right) \omega^{2}+j \omega R\left(C_{p}+C_{m}\right)}
\end{gathered}
$$

where $\mathbf{U}$ and $\mathbf{V}$ represent the input voltage and the actuation voltage across the parallel-plate actuator in the frequency domain, respectively; $\mathbf{C}$ represents total capacitance of the parallel-plate actuator, the sum of $\mathbf{C}_{\mathbf{m}}$ and $\mathbf{C}_{\mathbf{p}}$. In order to derive the voltage gain, we evaluate the frequency response of the resonant drive circuit, where $\mathbf{C}_{\mathbf{0}}, \boldsymbol{\omega}_{\mathbf{0}}, \mathbf{r}$, and $\mathbf{Q}_{\mathbf{f}}$ are the initial capacitance of the parallel-plate actuator, the frequency of the input signal that is fixed at the initial resonant frequency of the resonant drive circuit, the ratio of the parasitic capacitance $\mathbf{C}_{\mathbf{p}}$ to the initial capacitance of the parallel-plate actuator $\mathbf{C}_{\mathbf{0}}$, and the initial quality factor of the resonant drive circuit, respectively.

$$
\omega_{0}=\frac{1}{\sqrt{L C_{0}(r+1)}}, \quad r=\frac{C_{p}}{C_{0}}, \quad Q_{f}=\sqrt{\frac{L}{R^{2} C_{0}(r+1)}}
$$

Suppose that the frequency of the input AC voltage source is equal to the initial resonant frequency of the resonant drive circuit, remains constant and is much higher than the mechanical resonant frequency of the parallel-plate actuator, we evaluate and derive the voltage gain of the resonant drive circuit in terms of the initial quality factor $\mathbf{Q}_{\mathrm{f}}$, the parasitic capacitance $\mathbf{C}_{\mathrm{p}}$, and the capacitance of the parallel-plate actuator $\mathbf{C}_{\mathbf{m}}$ through the frequency response presented in Eq. 3.8: 


$$
\begin{aligned}
\left.H(j \omega)\right|_{\omega=\omega_{0}} & =\frac{1}{1-\frac{C_{p}+C_{m}}{C_{0}(r+1)}+j \frac{R\left(C_{p}+C_{m}\right)}{\sqrt{L C_{0}(r+1)}}} \\
& =\frac{1}{\frac{C_{0}-C_{m}}{C_{0}(r+1)}+j \frac{R\left(C_{p}+C_{m}\right)}{\sqrt{L C_{0}(r+1)}}} \\
& =\frac{C_{0}(r+1)}{C_{0}-C_{m}+j\left(C_{p}+C_{m}\right) \sqrt{\frac{R^{2} C_{0}(r+1)}{L}}} \\
& =\frac{Q_{f} C_{0}(r+1)}{Q_{f}\left(C_{0}-C_{m}\right)+j\left(C_{p}+C_{m}\right)}
\end{aligned}
$$

Discounting the fringe field of the parallel-plate actuator, its capacitance $\mathbf{C}_{\mathbf{m}}$ has a simple algebraic expression in terms of its normalized displacement $\mathbf{x}$ shown in Eq. 3.9:

$$
C_{m}=\frac{\varepsilon A}{g_{0}-X}=\frac{\varepsilon A}{g_{0}(1-x)}=\frac{C_{0}}{1-x}
$$

Using Eq. 3.9, we further simplify Eq. 3.8 into Eq. 3.10 representing the frequency response of the parallel-plate actuator coupled with the resonant drive circuit in terms of the displacement rather than the capacitance of the parallel-plate actuator:

$$
\begin{aligned}
H\left(j \omega_{0}\right) & =\frac{Q_{f} C_{0}(r+1)}{Q_{f}\left(C_{0}-\frac{C_{0}}{1-x}\right)+j C_{0}\left(r+\frac{1}{1-x}\right)} \\
& =\frac{Q_{f}(r+1)}{Q_{f}\left(\frac{-x}{1-x}\right)+j\left(\frac{r(1-x)+1}{1-x}\right)} \\
& =\frac{Q_{f}(r+1)(1-x)}{-Q_{f} x+j(r(1-x)+1)}
\end{aligned}
$$

The voltage gain of the resonant drive circuit is derived from the frequency response of Eq. 3.10 at the initial resonant frequency of the resonant drive circuit and is presented in Eq. 3.11, which follows the envelope of the AC actuation voltage. The phase delay caused by the displacement of the parallelplate actuator is also derived from Eq. 3.10 and is presented in Eq. 3.12, which provides a one-to-one mapping between the phase delay and the displacement of the parallel-plate actuator, and is used to measure the displacement of the parallel-plate actuator in Section 3.5.

$$
\left|H\left(j \omega_{0}\right)\right|=\frac{Q_{f}(r+1)(1-x)}{\sqrt{Q_{f}{ }^{2} x^{2}+(r(1-x)+1)^{2}}}
$$




$$
\angle H\left(j \omega_{0}\right)=-\tan ^{-1}\left(\frac{(r(1-x)+1)}{-Q_{f} x}\right)
$$

Because the parallel-plate actuator is too sluggish to follow a high frequency AC actuation voltage amplified by the resonant drive circuit, it only responds to the RMS (Root Mean Squared) value of the actuation voltage. Using the voltage gain in Eq. 3.11, we derive the RMS value of the AC actuation voltage presented in Eq. 3.13:

$$
V_{r m s}=\frac{1}{\sqrt{2}} \frac{Q_{f}(r+1)(1-x)}{\sqrt{Q_{f}^{2} x^{2}+(r(1-x)+1)^{2}}} u
$$

Substituting the actuation voltage $\mathbf{V}$ in Eq. 3.4 with $\mathbf{V}_{\text {rms }}$ in Eq. 3.13, we simplify the $4^{\text {th }}$ order system of the parallel-plate actuator coupled with the resonant drive circuit into the $2^{\text {nd }}$ order system with the algebraic constraint presented in Eq. 3.14:

$$
m \ddot{x}+b \dot{x}+k x=\frac{1}{4} \frac{\varepsilon A}{g_{0}^{3}} \frac{Q_{f}^{2}(r+1)^{2} u^{2}}{\left(Q_{f}^{2} x^{2}+(r(1-x)+1)^{2}\right)}
$$

Normalizing Eq. 3.14 with the conventional pull-in voltage of the parallel-plate actuator in Eq. 3.15 [7], we further simplify Eq. 3.14 into Eq. 3.16, which represents a normalized, non-dimensional governing equation of the parallel-plate actuator driven by the resonant drive circuit controlled by the amplitude modulation:

$$
\begin{gathered}
u=V_{p u l l-i n} \bar{u}=\sqrt{\frac{8 k g_{0}^{3}}{27 \varepsilon A} \bar{u}} \\
\ddot{x}+2 \xi \omega_{m} \dot{x}+\omega_{m}^{2} x=\frac{2}{27} \omega_{m}^{2} \frac{Q_{f}^{2}(r+1)^{2} \bar{u}^{2}}{\left(Q_{f}^{2} x^{2}+(r(1-x)+1)^{2}\right)}
\end{gathered}
$$

where $\overline{\mathbf{u}}$ denotes the normalized input voltage with respect to the conventional pull-in voltage of the parallel-plate actuator; $\boldsymbol{\xi}$ represents the mechanical damping ratio of the parallel-plate actuator; $\boldsymbol{\omega}_{\mathrm{m}}$ stands for the mechanical natural frequency of the parallel-plate actuator.

\subsection{Stability Analysis of Parallel-Plate Actuator Driven by Resonant Drive} Circuit Controlled by Amplitude Modulation

Because the velocity and acceleration of the parallel-plate actuator are zero at its fixed points, we further simplify Eq. 3.16 into Eq. 3.17 to determine its fixed points and their stability as thus:

$$
\omega_{m}^{2} x=\frac{2}{27} \omega_{m}^{2} \frac{Q_{f}^{2}(r+1)^{2} \bar{u}^{2}}{\left(Q_{f}^{2} x^{2}+(r(1-x)+1)^{2}\right)}
$$


Solving Eq. 3.17 in terms of the normalized displacement $\boldsymbol{x}$ and the normalized input voltage $\overline{\mathbf{u}}$, we determine the fixed points of the parallel-plate actuator coupled with the resonant drive circuit and their stability as thus:

$$
\begin{aligned}
F_{n e t} & =F_{m e}+F_{e s} \\
& =-\omega_{m}{ }^{2} x+\frac{2}{27} \omega_{m}{ }^{2} \frac{Q_{f}{ }^{2}(r+1)^{2} \bar{u}^{2}}{\left(Q_{f}{ }^{2} x^{2}+(r(1-x)+1)^{2}\right)} \\
& =\omega_{m}{ }^{2}\left(-x+\frac{2}{27} \frac{Q_{f}{ }^{2}(r+1)^{2} \bar{u}^{2}}{\left(Q_{f}{ }^{2} x^{2}+(r(1-x)+1)^{2}\right)}\right)
\end{aligned}
$$

where the mechanical restoring force $\mathbf{F}_{\mathbf{m e}}$ is equal to the spring force in Eq. 3.17 and the electrostatic force $\mathbf{F}_{\text {es }}$ is equal to the right side of Eq. 3.17. Since the velocity and acceleration of the parallel-plate actuator are zero at the fixed points, we can find the fixed points $\mathbf{x}^{*}$ of the parallel-plate actuator by setting $\mathbf{F}_{\text {net }}$ to be zero. To evaluate the stability of the fixed point $\mathbf{x}^{*}$, we need to evaluate the derivative of $\mathbf{F}_{\text {net }}$ with respect to $\mathbf{x}$ at the fixed point $\mathbf{x}^{*}$ as thus:

$$
\begin{aligned}
& \text { if }\left.\frac{\partial F_{n e t}}{\partial x}\right|_{x=x^{*}}<0, \text { then } x^{*} \text { is stable; } \\
& \text { if }\left.\frac{\partial F_{n e t}}{\partial x}\right|_{x=x^{*}}>0, \text { then } x^{*} \text { is unstable; } \\
& \text { if }\left.\frac{\partial F_{n e t}}{\partial x}\right|_{x=x^{*}}=0, \text { then } x^{*} \text { is a saddle node. }
\end{aligned}
$$

The fixed points of the parallel-plate actuator are presented in Figure 3.2, when it is driven by conventional voltage control and the resonant drive circuit with the initial quality factor $\mathbf{Q}_{\mathrm{f}}$ of 50 and three different cases of the parasitic capacitance $\mathbf{C}_{\mathbf{p}}$. The parallel-plate actuator driven by conventional voltage control undergoes the saddle-node bifurcation (the pull-in phenomenon) at $1 / 3$ of its original gap, as presented in Figure 3.2. However, the fixed points of the parallel-plate actuator driven by the resonant drive circuit depend on the quality factor of the resonant drive circuit and the parasitic capacitance of the parallel-plate actuator. If the parasitic capacitance is small enough $\left(\mathbf{C}_{\mathbf{p}}=50 \mathbf{C}_{\mathbf{0}}\right.$ in Figure 3.2), compared to the quality factor of the resonant drive circuit, the parallel-plate actuator does not experience the saddle-node bifurcation, and we are able to displace the parallel-plate actuator over its entire gap. In other words, all of the fixed points of the parallel-plate actuator are stable. In the case of $\mathbf{C}_{\mathbf{p}}=100 \mathbf{C}_{\mathbf{0}}$ in Figure 3.2, the parallel-plate actuator has three fixed points: two stable fixed points separated by one unstable fixed point that causes hysteresis or path-dependence in the response of the parallel-plate actuator. As the parasitic capacitance increases, the bifurcation diagram of the parallel-plate actuator becomes similar to that of conventional voltage control: one stable fixed point in the lower branch and one unstable fixed point in the upper branch. However, we are still capable of driving the parallel-plate actuator at much lower voltage than the conventional pull-in voltage $\mathbf{V}_{\mathbf{p i}}$ : the pull-in voltage of the parallel-plate actuator coupled with the resonant drive circuit is roughly equal to $\mathbf{V}_{\mathbf{p i}} / \mathbf{Q}_{\mathbf{f}}$ in the presence of high parasitic capacitance $\left(\mathbf{C}_{\mathbf{p}}>500 \mathbf{C}_{\mathbf{0}}\right)$. The location of the saddle-node bifurcation of the parallel-plate actuator in terms of the parasitic capacitance and the initial quality factor are presented in Figure 3.3 as well. 


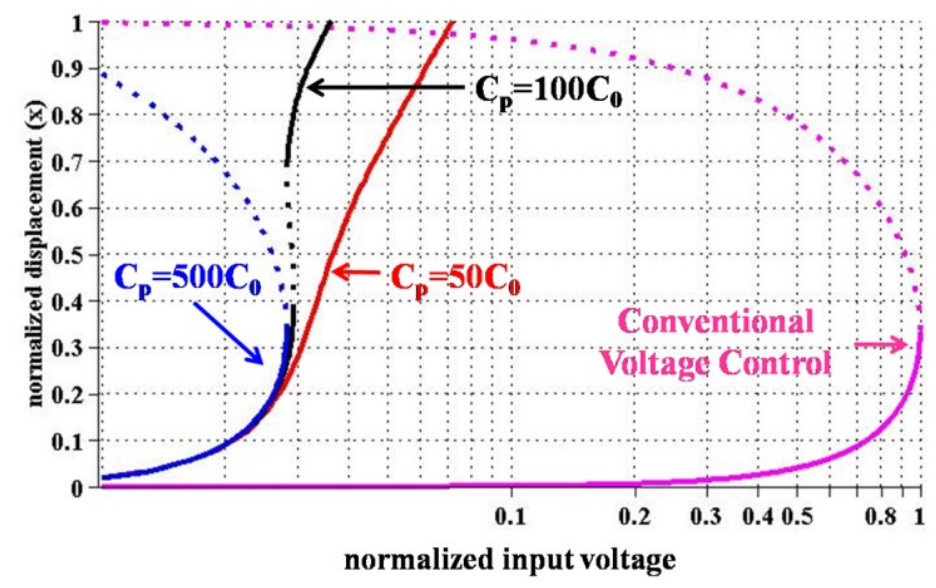

Figure 3.2 Fixed points of the parallel-plate actuator driven by conventional voltage control and the resonant drive circuit with the initial quality factor $\mathbf{Q}_{\mathrm{f}}=50$ and different parasitic capacitance: (a) $\mathbf{C}_{\mathbf{p}}=50 \mathbf{C}_{\mathbf{0}}$, (b) $\mathbf{C}_{\mathbf{p}}=100 \mathbf{C}_{\mathbf{0}}$, (c) $\mathbf{C}_{\mathbf{p}}=500 \mathbf{C}_{\mathbf{0}}$. The solid lines represent stable fixed points and the dotted lines depict unstable fixed points of the parallel-plate actuator.

It is well known that the open-loop charge control [7] can displace an electrostatic actuator over its entire gap in the absence of the parasitic capacitance. In reality, every electrostatic actuator comes with the parasitic capacitance no matter how small it is. Hence, the open-loop charge control also undergoes the saddle-node bifurcation even in the presence of small parasitic capacitance, as presented in Figure 3.3 showing the location of the pull-in points in terms of the parasitic capacitance.

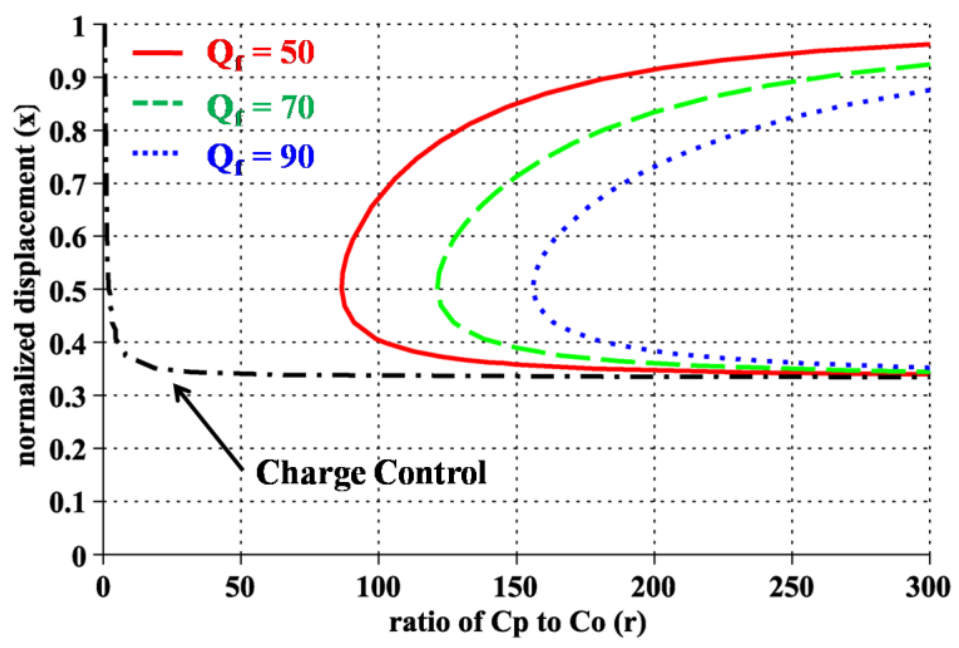

Figure 3.3 Location of the saddle-node bifurcation of the parallel-plate actuator driven by the resonant drive circuit with the initial quality factor: (a) $\mathbf{Q}_{\mathrm{f}}=50$, (b) $\mathbf{Q}_{\mathrm{f}}=70$, (c) $\mathbf{Q}_{\mathrm{f}}=90$, (d) openloop charge control represented by black dash-dot line. 
The parallel-plate actuator coupled with the resonant drive circuit is less sensitive to the parasitic capacitance due to its inherent negative feedback. In other words, even in the presence of the high parasitic capacitance, the resonant drive circuit is able to displace the parallel-plate actuator over its entire gap, if its quality factor is high enough. When the quality factor of the resonant drive circuit is not high enough, the parallel-plate actuator coupled with the resonant drive circuit does undergo the saddle-node bifurcation twice that opens up the region of the unstable fixed points depicted in Figure 3.4 .

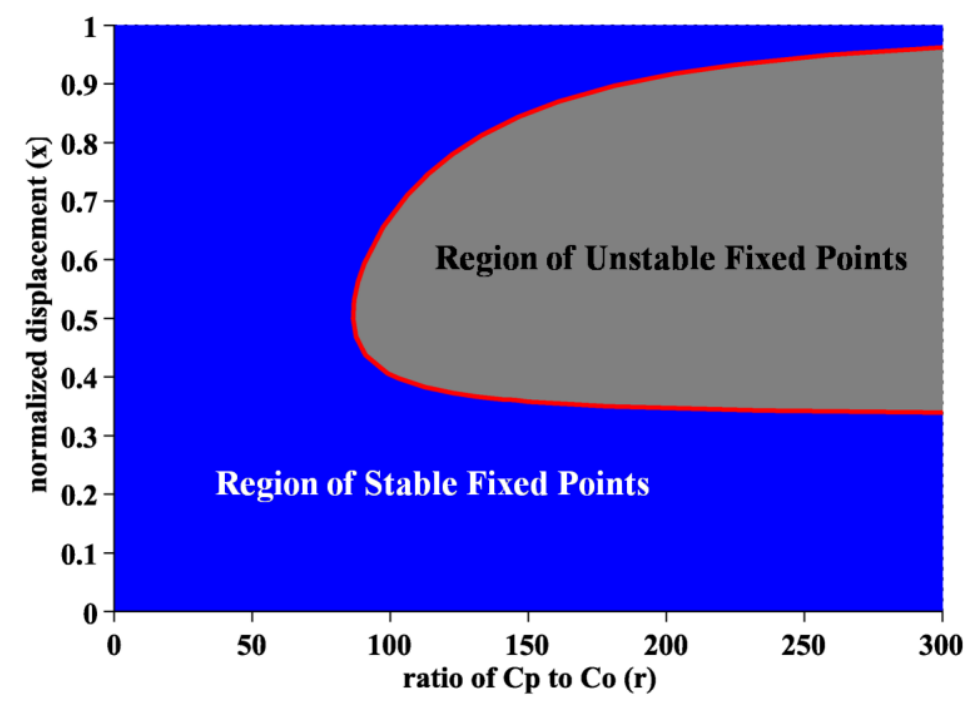

Figure 3.4 Regions of the stable fixed points and the unstable fixed points of the parallel-plate actuator driven by the resonant drive circuit with the quality factor $\mathbf{Q}_{\mathrm{f}}$ of 50: the red solid line represents the location of the saddle-node bifurcation.

\subsection{Analytical Model of Parallel-Plate Actuator Driven by Resonant Drive Circuit Controlled by Frequency Modulation}

In Section 3.1, we derive the analytical model of the parallel-plate actuator coupled with the resonant drive circuit, which is controlled by varying the amplitude of the low voltage AC input signal, while the frequency of the input voltage is fixed at the initial resonant frequency of the resonant drive circuit. In this section, we derive the analytical model of the same parallel-plate actuator coupled with the resonant drive circuit, when it is controlled by the frequency modulation. In other words, we vary the frequency of the input signal to displace the parallel-plate actuator while the amplitude of the input voltage to the resonant drive circuit remains constant. Hence, the same governing equations presented in Section 3.1 (from Eq. 3.1 to Eq. 3.7) are used to represent the parallel-plate actuator coupled with the resonant drive circuit. However, the frequency response of the resonant drive circuit presented in Eq. 3.19 differs from those in Section 3.1, because the frequency of the input signal is a control variable in this case: 


$$
|H(j \omega)|=\frac{1}{\sqrt{\left(1-L C \omega^{2}\right)^{2}+(R C \omega)^{2}}}
$$

Using Eq. 3.19, we derive the RMS value of the actuation voltage of the parallel-plate actuator as thus:

$$
V_{r m s}=\frac{1}{\sqrt{2}} \frac{u}{\sqrt{\left(1-L C \omega^{2}\right)^{2}+(R C \omega)^{2}}}
$$

Substituting Eq. 3.20 into Eq. 3.4, we determine the electrostatic force exerted on the parallel-plate actuator in terms of the frequency and the peak amplitude of the input voltage as thus:

$$
F_{e s}=\frac{\varepsilon A}{2 m g_{0}^{3}} \frac{V_{r m s}^{2}}{(1-x)^{2}}=\frac{\varepsilon A}{4 m g_{0}^{3}(1-x)^{2}} \frac{u^{2}}{\left(1-L C \omega^{2}\right)^{2}+(R C \omega)^{2}}
$$

Using the total capacitance represented by Eq. 3.22 and the normalized input frequency in terms of the initial resonant frequency of the resonant drive circuit, which is presented in Eq. 3.23, we further simplify the electrostatic force presented in Eq. 3.21 into Eq. 3.24:

$$
\begin{aligned}
& C=C_{m}+C_{p}=\frac{C_{0}}{1-x}+r C_{0}=C_{0}\left(\frac{1}{1-x}+r\right)=\frac{C_{0}}{1-x}(1+r(1-x)) \\
& \omega=\omega_{0} \bar{\omega}=\frac{1}{\sqrt{L C_{0}(r+1)}} \bar{\omega} \\
& F_{e s}=\frac{\varepsilon A}{4 m g_{0}{ }^{3}(1-x)^{2}} \frac{u^{2}}{\left(1-\frac{L C_{0}(1+r(1-x)) \omega^{2}}{1-x}\right)^{2}+\left(\frac{R C_{0}(1+r(1-x)) \omega}{1-x}\right)^{2}} \\
& =\frac{\varepsilon A}{4 m g_{0}{ }^{3}} \frac{u^{2}}{\left(1-x-L C_{0}(1+r(1-x)) \omega^{2}\right)^{2}+\left(R C_{0}(1+r(1-x)) \omega\right)^{2}} \\
& =\frac{\varepsilon A}{4 m g_{0}{ }^{3}} \frac{u^{2}}{\left(1-x-\frac{(1+r(1-x))}{(r+1)} \bar{\omega}^{2}\right)^{2}+\left(\frac{R C_{0}(1+r(1-x)) \bar{\omega}}{\sqrt{L C_{0}(r+1)}}\right)^{2}} \\
& =\frac{\varepsilon A}{4 m g_{0}{ }^{3}} \frac{u^{2}}{\frac{\left((1-x)(r+1)-(1+r(1-x)) \bar{\omega}^{2}\right)^{2}}{(r+1)^{2}}+\frac{(1+r(1-x))^{2} \bar{\omega}^{2}}{Q_{f}{ }^{2}(r+1)^{2}}} \\
& =\frac{\varepsilon A}{4 m g_{0}^{3}} \frac{Q_{f}{ }^{2}(r+1)^{2} u^{2}}{Q_{f}{ }^{2}\left((1-x)(r+1)-(1+r(1-x)) \bar{\omega}^{2}\right)^{2}+(1+r(1-x))^{2} \bar{\omega}^{2}}
\end{aligned}
$$

Using the normalized input voltage in terms of the conventional pull-in voltage [7] in Eq. 3.15, we further simplify Eq. 3.24 into Eq. 3.25: 


$$
F_{e s}=\frac{2}{27} \omega_{m}^{2} \frac{Q_{f}^{2}(r+1)^{2} \bar{u}^{2}}{Q_{f}^{2}\left((1-x)(r+1)-(1+r(1-x)) \bar{\omega}^{2}\right)^{2}+(1+r(1-x))^{2} \bar{\omega}^{2}}
$$

Hence, the normalized, non-dimensional governing equation of the parallel-plate actuator coupled with the resonant drive circuit controlled by the frequency modulation is represented by Eq. 3.26:

$$
\ddot{x}+2 \xi \omega_{m} \dot{x}+\omega_{m}{ }^{2} x=\frac{2}{27} \omega_{m}{ }^{2} \frac{Q_{f}{ }^{2}(r+1)^{2} \bar{u}^{2}}{Q_{f}^{2}\left((1-x)(r+1)-(1+r(1-x))^{2}\right)^{2}+(1+r(1-x))^{2} \bar{\omega}^{2}}
$$

where $\xi$ represents the damping ratio of the parallel-plate actuator; $\boldsymbol{\omega}_{\mathrm{m}}$ denotes the mechanical natural frequency of the parallel-plate actuator; $\overline{\mathbf{u}}$ represents the normalized input voltage; and $\overline{\boldsymbol{\omega}}$ denotes the normalized input frequency in terms of the initial resonant frequency $\boldsymbol{\omega}_{\mathbf{0}}$.

\subsection{Stability Analysis of Parallel-Plate Actuator Driven by Resonant Drive Circuit Controlled by Frequency Modulation}

Using the same method described in Section 3.2, we derive the net force in Eq. 3.27 to determine the fixed points of the parallel-plate actuator coupled with the resonant drive circuit, which is controlled by the frequency modulation:

$$
\begin{aligned}
F_{n e t} & =F_{m e}+F_{e s} \\
& =-\omega_{m}{ }^{2} x+\frac{2}{27} \omega_{m}{ }^{2} \frac{Q_{f}^{2}(r+1)^{2} \bar{u}^{2}}{Q_{f}{ }^{2}\left((1-x)(r+1)-(1+r(1-x)) \bar{\omega}^{2}\right)^{2}+(1+r(1-x))^{2} \bar{\omega}^{2}} \\
& =\omega_{m}{ }^{2}\left(-x+\frac{2}{27} \frac{Q_{f}{ }^{2}(r+1)^{2} \bar{u}^{2}}{{Q_{f}}^{2}\left((1-x)(r+1)-(1+r(1-x)) \bar{\omega}^{2}\right)^{2}+(1+r(1-x))^{2} \bar{\omega}^{2}}\right)
\end{aligned}
$$

Solving Eq. 3.27, we determine the fixed points of the parallel-plate actuator and their stability, when it is driven by the resonant drive circuit that is controlled by frequency modulation, as presented in Figure 3.5, where the solid lines represent the stable fixed points of the parallel-plate actuator and the dotted lines denote the unstable fixed points, while the frequency of the input signal to the resonant drive circuit varies. As presented in Figure 3.5, the parallel-plate actuator exhibits completely different behavior, compared to its behavior as controlled by amplitude modulation in Figure 3.2. When the parasitic capacitance of the parallel-plate actuator is small enough $(\mathbf{r}<83)$ with the given quality factor of 50, we are able to displace the parallel-plate actuator over its entire gap as we sweep

the frequency of the input signal downward. However, with the same condition, the parallel-plate actuator undergoes the saddle-node bifurcation as we sweep the frequency of the input signal upward. The reason is that the instantaneous resonant frequency of the resonant drive circuit decreases as the parallel-plate actuator displaces toward its bottom electrode. When the frequency of the input signal locks into the reduced resonant frequency, the instantaneous voltage gain of the resonant drive circuit soars, leading to the pull-in. In the case of high parasitic capacitance and high input voltage, the parallel-plate actuator undergoes the saddle-node bifurcation, regardless of the direction of the 
frequency sweep. In order to understand how each parameter affects the fixed points of the parallelplate actuator, we evaluate the fixed points while varying one parameter with other parameters fixed, as presented in Figure 3.6, Figure 3.7, and Figure 3.8, where the fixed points within the shaded regions are unstable, and otherwise they are stable.

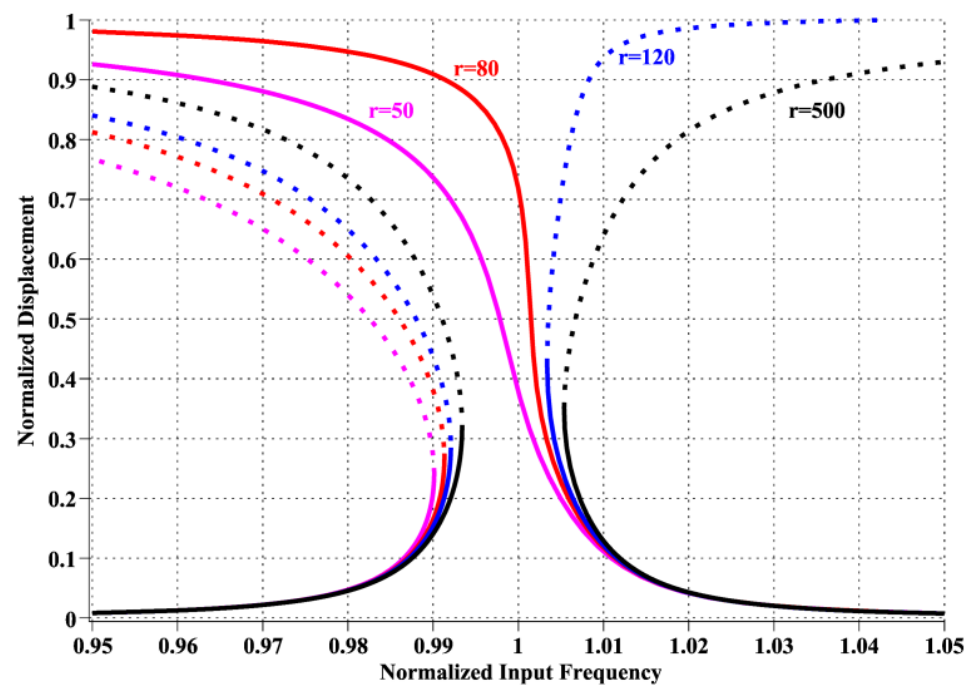

Figure 3.5 Fixed points of the parallel-plate actuator driven by the resonant drive circuit that is controlled by the frequency modulation with the parameters of $\mathbf{Q}_{\mathrm{f}}=50$ and $\overline{\mathbf{u}}=0.033$ : the solid lines represent the stable fixed points; and the dotted lines denote the unstable fixed points.
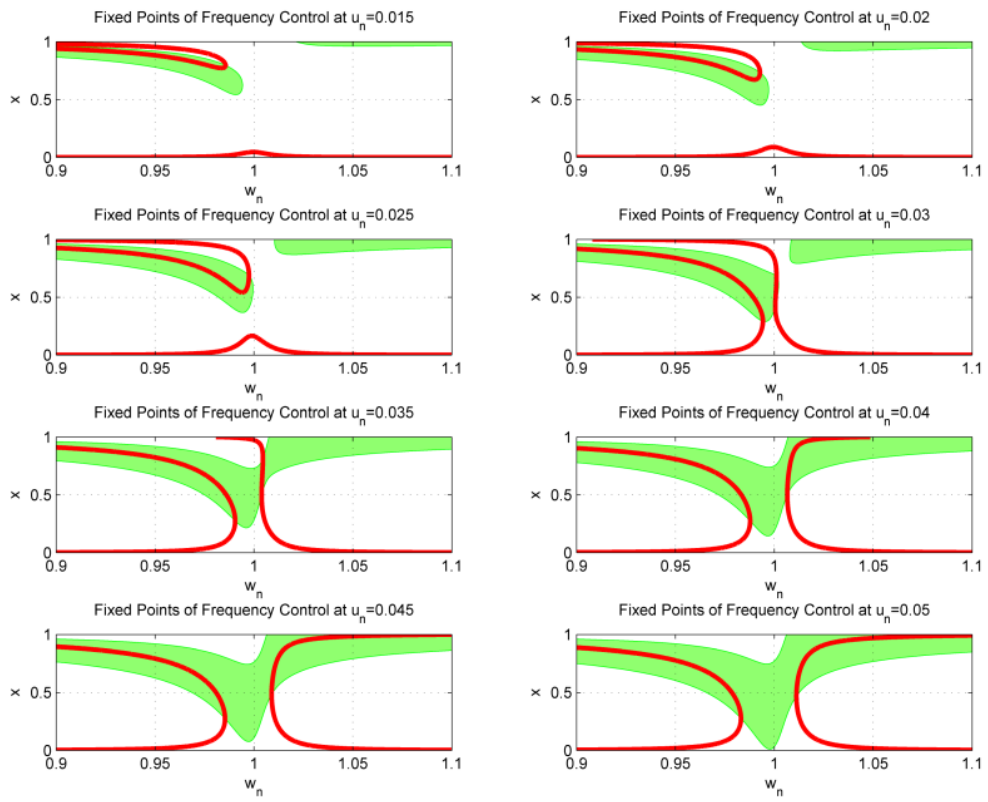

Figure 3.6 Fixed points of the parallel-plate actuator coupled with the resonant drive circuit controlled by the frequency modulation at various input voltages with $\mathbf{Q}_{\mathbf{f}}=50$ and $\mathbf{r}=100$. 
In Figure 3.6, we present how the fixed points of the parallel-plate actuator vary as the normalized input voltage increases. At the low input voltage of $\overline{\mathbf{u}}=0.015,0.02$ and 0.025 , the parallel-plate actuator does not displace much over the entire range of the input frequency. However, as the normalized input voltage increases, the saddle-node bifurcation occurs, splitting the entire parameter space into two regions: the stable and unstable regions.

In Figure 3.7, we present the fixed points of the parallel-plate actuator as the quality factor of the resonant drive circuit varies. With the normalized input voltage of $\overline{\mathbf{u}}=0.03$ and the parasitic capacitance of $\mathbf{C}_{\mathbf{p}}=200 \mathbf{C}_{\mathbf{0}}$, there are always the saddle-node bifurcations, regardless of the direction of the frequency sweep. However, the region of unstable fixed points diminishes as the quality factor increases.

As the parasitic capacitance of the parallel-plate actuator increases and other parameters remain constant, the fixed points of the parallel-plate actuator are presented in Figure 3.8. With the normalized input voltage of $\overline{\mathbf{u}}=0.03$ and the quality factor of $\mathbf{Q}_{\mathrm{f}}=50$, the parallel-plate actuator does not undergo the saddle-node bifurcation at the low parasitic capacitance of $\mathbf{C}_{\mathbf{p}}=50 \mathbf{C}_{\mathbf{0}}$ during the downward frequency sweep. Moreover, the parallel-plate actuator has three fixed points in the left region of the parameter space and one stable fixed point in the right region of the parameter space; two stable fixed points separated by one unstable fixed point exist in the left region of the parameter space and one stable fixed point occurs in the right region, as long as $\mathbf{C}_{\mathbf{p}}<120 \mathbf{C}_{\mathbf{0}}$.
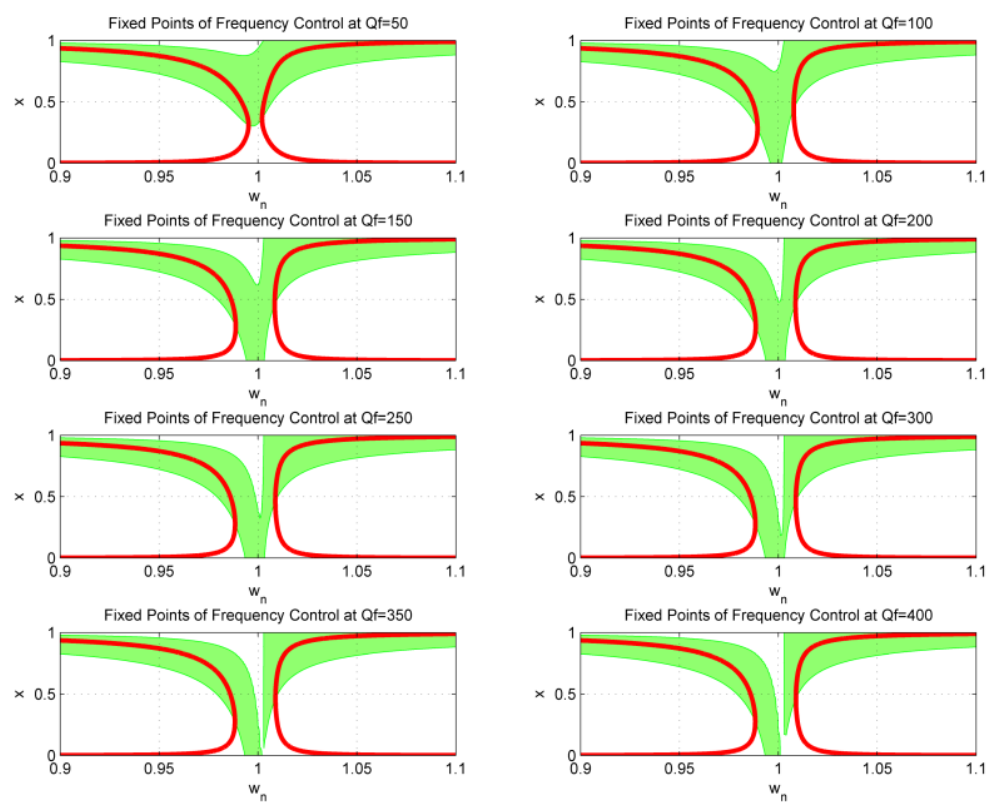

Figure 3.7 Fixed points of the parallel-plate actuator coupled with the resonant drive circuit that is controlled by the frequency modulation at various quality factors with the fixed input voltage $\overline{\mathbf{u}}=0.03$ and $\mathbf{r}=200$. 

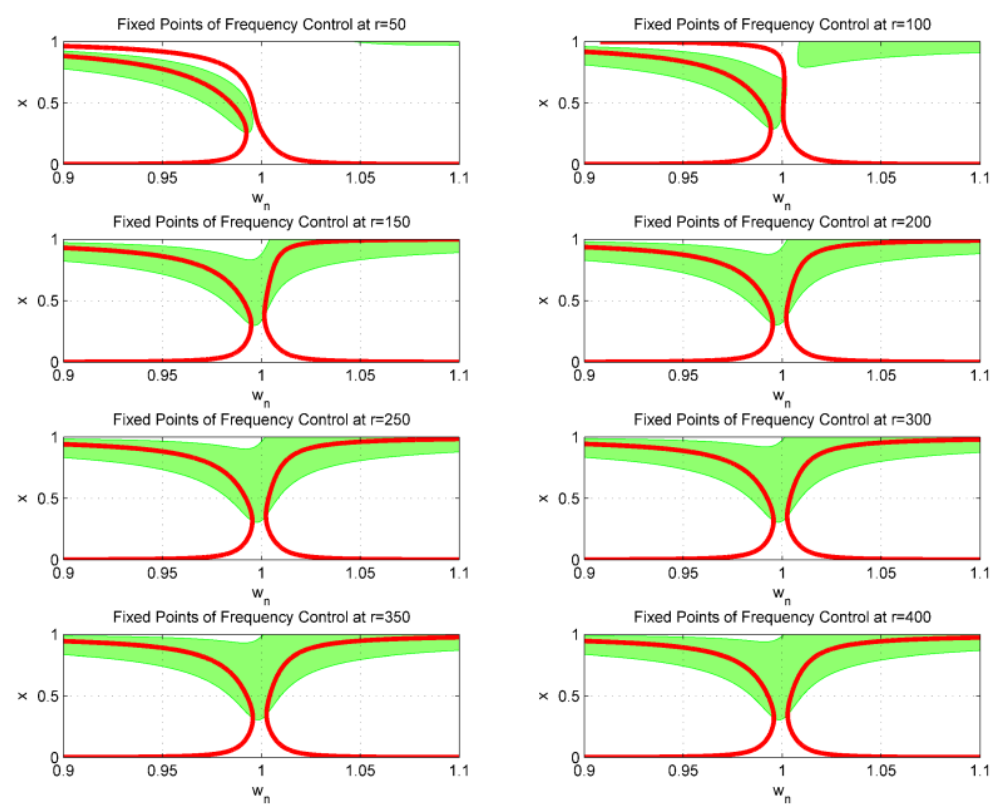

Figure 3.8 Fixed points of the parallel-plate actuator coupled with the resonant drive circuit that is controlled by the frequency modulation in the presence of various parasitic capacitances with the fixed input voltage $\overline{\mathbf{u}}=0.03$ and the quality factor $\mathbf{Q}_{\mathrm{f}}=50$.

\subsection{Displacement Measurement of Parallel-Plate Actuator Coupled with Resonant Drive Circuit}

One of the disadvantages of an electrostatic actuator is that it is difficult to measure its displacement, due to its small size and limitation of its fabrication methods. In this section, we present a new method of the displacement measurement by sensing the phase change of the actuation voltage or the current through the resonant drive circuit with reference to the $\mathrm{AC}$ input voltage using a phase detector. The input signal, frequency of which is equal to the initial electrical resonance of the resonant drive circuit, can be considered as an unmodulated carrier signal. As an electrostatic actuator displaces toward its bottom electrode, its capacitance increases, and the electrical resonant frequency of the resonant drive circuit decreases. This deviation in the resonant frequency causes less voltage gain and more phase delay in the actuation voltage. In other words, it can be viewed that an electrostatic actuator modulates the input signal with its displacement, resulting in the actuation voltage modulated by its displacement. By demodulating the actuation voltage in terms of the voltage gain or the phase delay, we are able to measure the change in its capacitance to determine the displacement of the parallel-plate actuator coupled with the resonant drive circuit. The voltage gain can be measured by an envelope detector, the simplified schematic of which is presented in Figure 3.9, and the phase delay of the actuation voltage can be detected by mixing the input signal with the actuation voltage, which is done by a phase detector presented in Figure 3.12. Since the actuation voltage can be quite high, we need an attenuator to reduce its amplitude before the actuation voltage is demodulated by an envelope detector or a phase detector. However, an attenuator causes reduction 
in the quality factor of the resonant drive circuit by adding more parasitic capacitance or more resistance in parallel with an electrostatic actuator. Hence, it is better to demodulate the current signal rather than the actuation voltage, for the current can be easily measured by a current-sensing amplifier or a current transformer without much loss in the resonant drive circuit. The current through the resonant drive circuit is also modulated by the displacement of an electrostatic actuator in the same way that the actuation voltage is. However, the amount of the current through the resonant drive circuit is in an order of $\mu \mathrm{A}$ or $\mathrm{mA}$, depending on the resonant frequency and the quality factor. Thus, the current signal needs to be amplified before the demodulation.

In the presence of high parasitic capacitance, the dynamic range of an envelope detector and a phase detector must increase in order to measure small change in the voltage gain or the phase delay at the same resolution, because, even though the resolution of the measurement remains constant, the range of the capacitance we have to measure is bigger with higher parasitic capacitance. It turns out that the phase delay is more sensitive to the displacement than the voltage gain is. Furthermore, the phase delay provides one-to-one mapping to the displacement without any ambiguity while the voltage gain does not even in the presence of low parasitic capacitance.

\subsubsection{Displacement Measurement through Phase Delay of Actuation Voltage with}

\section{Respect to Input Voltage}

As shown in Eq. 3.11, the voltage gain of the resonant drive circuit depends on the displacement of the parallel-plate actuator; the initial quality factor $\mathbf{Q}_{\mathbf{f}}$ and the ratio of the parasitic capacitance $\mathbf{C}_{\mathbf{p}}$ to the initial capacitance $\mathbf{C}_{\mathbf{0}}$ of the parallel-plate actuator $r$ are rather constant, being determined through the parameter identification. Thus, we might be able to determine the displacement through the voltage gain that is measured by an envelope detector presented in Figure 3.9. However, the voltage gain has very low sensitivity to the displacement in the presence of high parasitic capacitance and does not provide one-to-one mapping to the displacement even with low parasitic capacitance as shown in Figure 3.10.

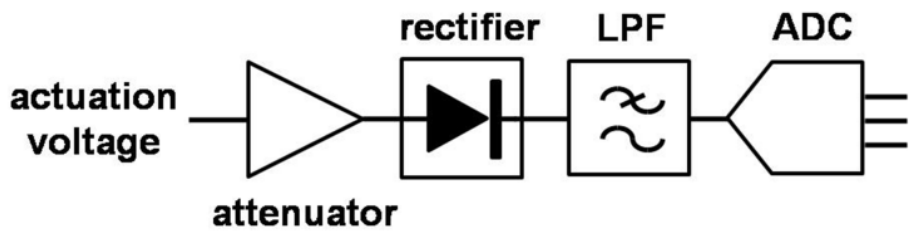

Figure 3.9 Envelope detector to measure the peak amplitude of the actuation voltage with an analog-digital converter (ADC)

However, the phase delay of the actuation voltage represented by Eq. 3.12 has better sensitivity to the displacement in the presence of high parasitic capacitance and provides one-to-one mapping to the displacement over a wide range of the parasitic capacitance as depicted in Figure 3.11. Solving Eq. 3.12 in terms of the normalized displacement $\mathbf{x}$, we obtain the algebraic expression of the displacement in terms of the phase delay of the actuation voltage as presented in Eq. 3.28: 


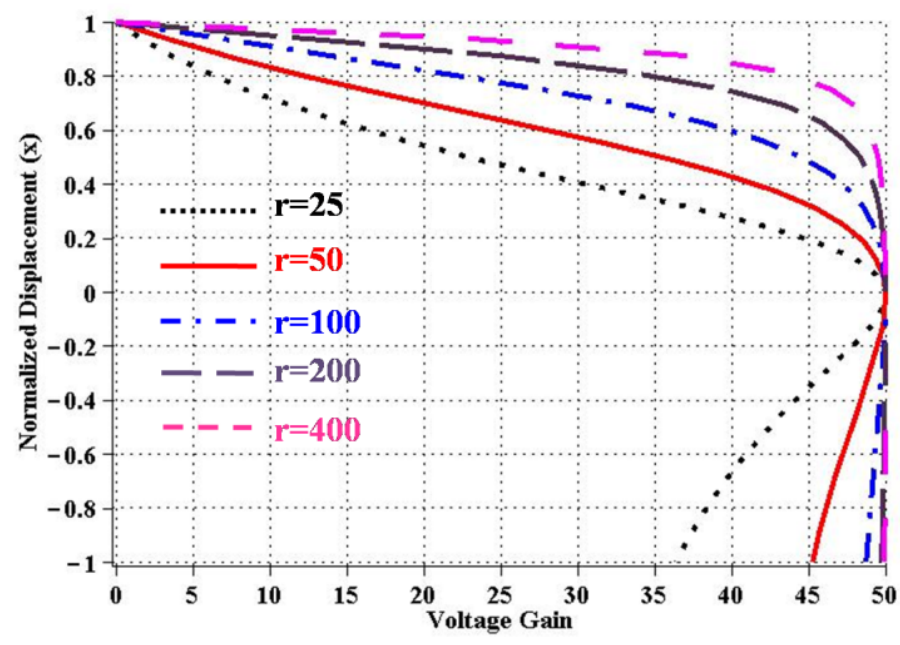

Figure 3.10 Normalized displacement of the parallel-plate actuator in terms of the voltage gain with the initial quality factor $\mathbf{Q}_{\mathrm{f}}=50$, and the ratio of $\mathbf{C}_{\mathbf{p}}$ to $\mathbf{C}_{\mathbf{0}}, \mathbf{r}=25, \mathbf{r}=50, \mathbf{r}=100, \mathbf{r}=200$, and $\mathbf{r}=400$.

$$
x=\frac{r+1}{Q_{f} \tan \left(\angle H\left(j \omega_{0}\right)\right)+r}
$$

Hence, we are able to determine the displacement of the parallel-plate actuator by measuring the phase difference between two sinusoidal signals of the same frequency with a phase detector, which is, in essence, a frequency mixer, whose simplified schematic is presented in Figure 3.12.

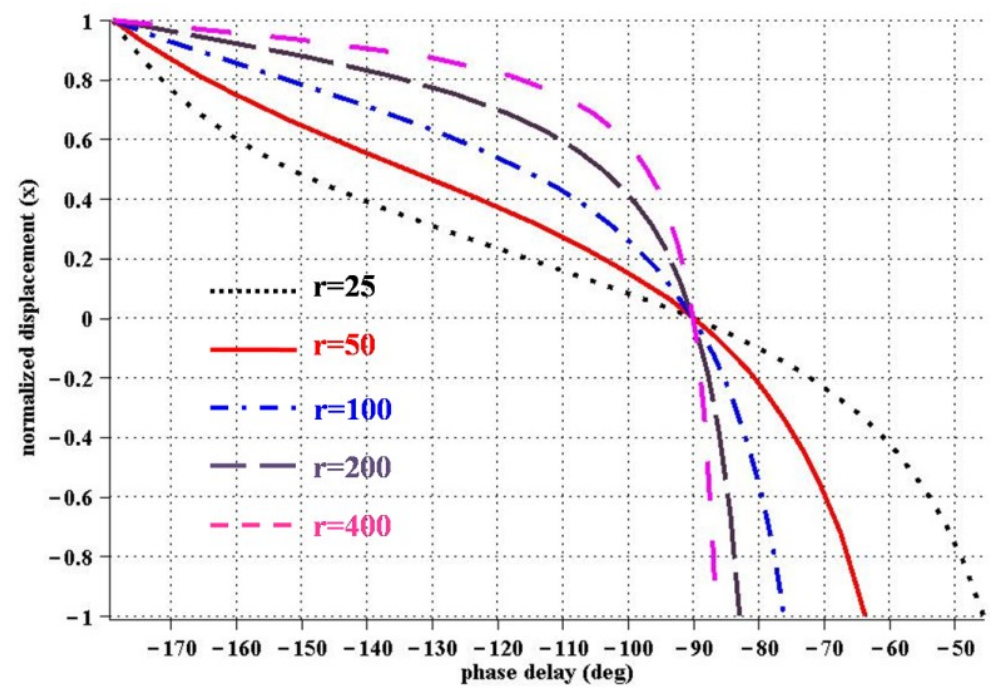

Figure 3.11 Normalized displacement of the parallel-plate actuator in terms of the phase delay of the actuation voltage with reference to the input voltage in the case of the initial quality factor $\mathbf{Q}_{\mathrm{f}}=50$, and the ratio of $\mathbf{C}_{\mathbf{p}}$ to $\mathbf{C}_{\mathbf{0}}, \mathbf{r}=25, \mathbf{r}=50, \mathbf{r}=100, \mathbf{r}=200$, and $\mathbf{r}=400$. 


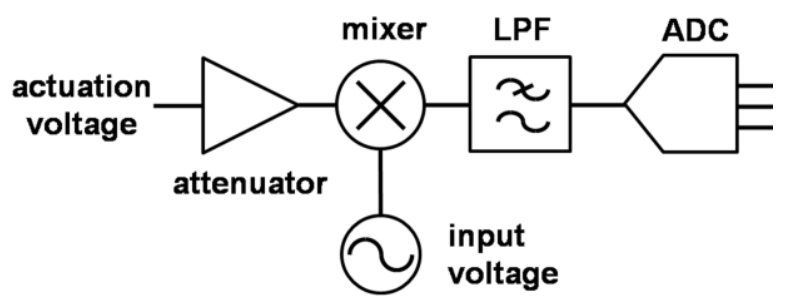

Figure 3.12 Schematic of the phase detector to measure the phase delay of the actuation voltage with respect to the input signal.

\subsubsection{Displacement Measurement through Phase Change between Input Current and Input Voltage}

Because the actuation voltage of the electrostatic actuator is usually too high to be measured by contemporary low voltage circuit, it must be attenuated by an attenuator, which might be a simple voltage divider. However, a voltage divider placed in parallel with an electrostatic actuator can induce additional loss, reducing the quality factor of the resonant drive circuit. To circumvent this problem, we measure the phase delay between the input voltage and the input current, which can be measured by a current-sensing amplifier, or a current transformer, which introduces an additional constant phase offset due to its inductive nature. To find out the relationship between the input current and the input voltage, we derive the conductance of the resonant drive circuit in Eq. 3.29. The conductance of the resonant drive circuit at the initial electrical resonance $\boldsymbol{\omega}_{\mathbf{0}}$ is evaluated as shown in Eq. 3.30.

$$
\begin{aligned}
& G(s)=\frac{I(s)}{U(s)}=\frac{C s}{L C s^{2}+R C s+1}=\frac{s}{L s^{2}+R s+\frac{1}{C}} \\
& \left.G(j \omega)\right|_{\omega=\omega_{0}}=\frac{j \omega_{0}}{\frac{1}{C}-L \omega_{0}^{2}+j \omega_{0} R} \\
& =\frac{j \frac{1}{\sqrt{L C_{0}(r+1)}}}{\frac{1}{C_{p}+C_{m}}-\frac{1}{C_{0}(r+1)}+j \sqrt{\frac{R^{2}}{L C_{0}(r+1)}}} \\
& =\frac{j \frac{1}{\sqrt{L C_{0}(r+1)}}}{\frac{C_{0}-C_{m}}{\left(C_{p}+C_{m}\right) C_{0}(r+1)}+j \sqrt{\frac{R^{2}}{L C_{0}(r+1)}}} \\
& =\frac{j \sqrt{\frac{C_{0}(r+1)}{L}}}{C_{0}-C_{m}+j\left(C_{p}+C_{m}\right) \sqrt{\frac{R^{2} C_{0}(r+1)}{L}}} \\
& =\frac{1}{R} \frac{j\left(C_{p}+C_{m}\right)}{Q_{f}\left(C_{0}-C_{m}\right)+j\left(C_{p}+C_{m}\right)}
\end{aligned}
$$


Since the capacitance of the parallel-plate actuator has a simple algebraic expression in terms of the displacement shown in Eq. 3.9, the conductance of the resonant drive circuit at its initial resonance is further simplified into Eq. 3.31 as shown thus:

$$
\begin{aligned}
\left.G(j \omega)\right|_{\omega=\omega_{0}} & =\frac{1}{R} \frac{j\left(C_{p}+\frac{C_{0}}{1-x}\right)}{Q_{f}\left(C_{0}-\frac{C_{0}}{1-x}\right)+j\left(C_{p}+\frac{C_{0}}{1-x}\right)} \\
& =\frac{1}{R} \frac{j C_{0}\left(r+\frac{1}{1-x}\right)}{Q_{f} C_{0}\left(1-\frac{1}{1-x}\right)+j C_{0}\left(r+\frac{1}{1-x}\right)} \\
& =\frac{j(r(1-x)+1)}{-R Q_{f} x+j R(r(1-x)+1)} \\
& =\frac{1}{R+j \frac{R Q_{f} x}{(r(1-x)+1)}}
\end{aligned}
$$

Its magnitude response and phase response are presented in Eq. 3.32 and Eq. 3.33, respectively.

$$
\begin{aligned}
& \left|G\left(j \omega_{0}\right)\right|=\frac{1}{\sqrt{R^{2}+\frac{\left(R Q_{f} x\right)^{2}}{(r(1-x)+1)^{2}}}} \\
& \angle G\left(j \omega_{0}\right)=-\tan ^{-1}\left(\frac{Q_{f} x}{r(1-x)+1}\right)
\end{aligned}
$$

Solving Eq. 3.33 in terms of the normalized displacement, we obtain the algebraic expression of the normalized displacement of the parallel-plate actuator as thus:

$$
x=\frac{(r+1) \tan \left(\angle G\left(j \omega_{0}\right)\right)}{r \tan \left(\angle G\left(j \omega_{0}\right)\right)-Q_{f}}
$$

As explained previously, the initial quality factor $\mathbf{Q}_{\mathbf{f}}$ of the resonant drive circuit and the ratio of the parasitic capacitance $\mathbf{C}_{\mathbf{p}}$ to the initial capacitance $\mathbf{C}_{\mathbf{0}}$ of the parallel-plate actuator can be determined through the parameter identification. Then, we are able to determine the displacement of the parallelplate actuator by measuring the phase delay between the input current and the input voltage, as presented in Figure 3.13. 


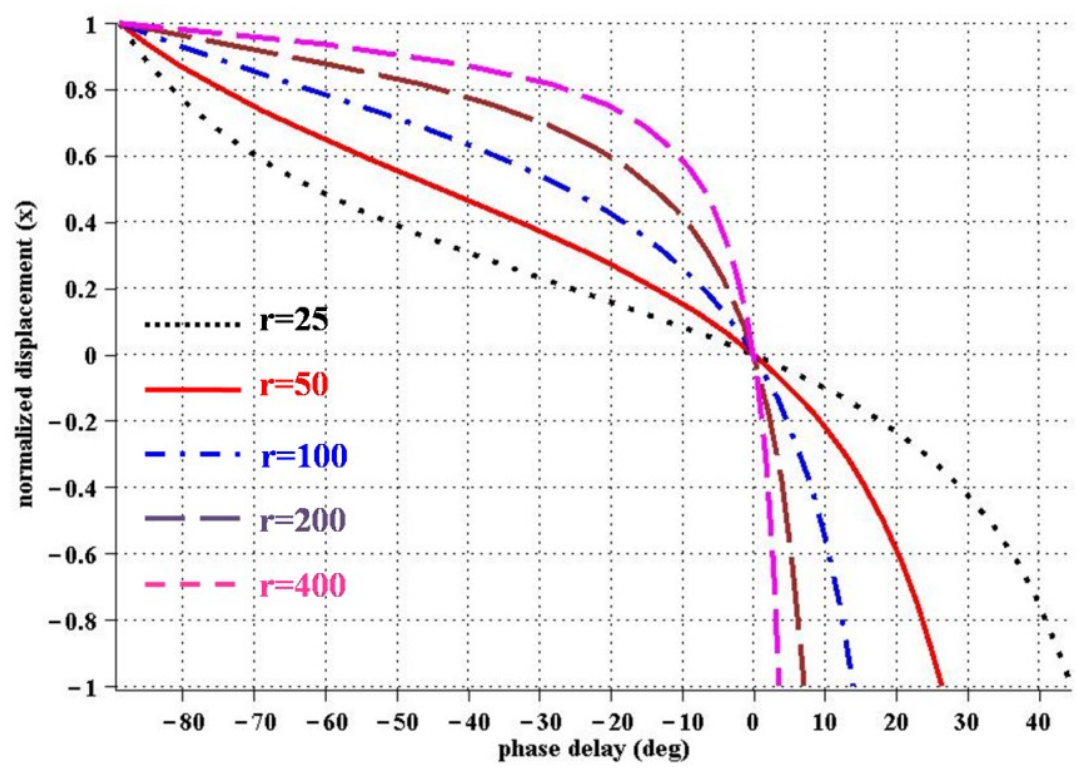

Figure 3.13 Normalized displacement of the parallel-plate actuator in terms of the phase delay of the input current with reference to the input voltage in case of the initial quality factor $\mathbf{Q}_{\mathrm{f}}=50$, and the ratio of $\mathbf{C}_{\mathbf{p}}$ to $\mathbf{C}_{\mathbf{0}}, \mathbf{r}=25, \mathbf{r}=50, \mathbf{r}=100, \mathbf{r}=200$, and $\mathbf{r}=400$.

\subsection{Numerical Model of Parallel-plate Actuator Coupled with Resonant Drive Circuit}

In order to validate the analytical model of the parallel-plate actuator driven by the resonant drive circuit and determine its response in the time domain, we create its numerical model in Matlab/Simulink. First, we build the numerical models of the parallel-plate actuator and the resonant drive circuit that are not coupled to each other to verify the integrity of each numerical model. Second, we couple them together through the state variables: the displacement and the actuation voltage of the parallel-plate actuator. Then, we perform the numerical integration at various input voltage to determine the step responses of the parallel-plate actuator coupled with the resonant drive circuit, whose numerical model is presented in Figure 3.14. Its governing equations are presented in Eq. 3.1, Eq. 3.2, and Eq. 3.3. Its physical parameters used in the numerical simulation are presented in Table 3.1. Its step response at the input voltage of $\mathbf{u}=0.7 \mathrm{~V}$ or the corresponding normalized input voltage of $\overline{\mathbf{u}}=0.0342$, is presented in Figure 3.16.

Even though its step response is well matched with the analytical result presented in both Figure 3.2 and Figure 3.3, this numerical model is computationally expensive, because it requires very small time step in order to simulate the resonant drive circuit properly. Hence, we create the simplified numerical model presented in Figure 3.15, whose dynamics is described by Eq. 3.16. Its step response is presented in Figure 3.16 as well. 


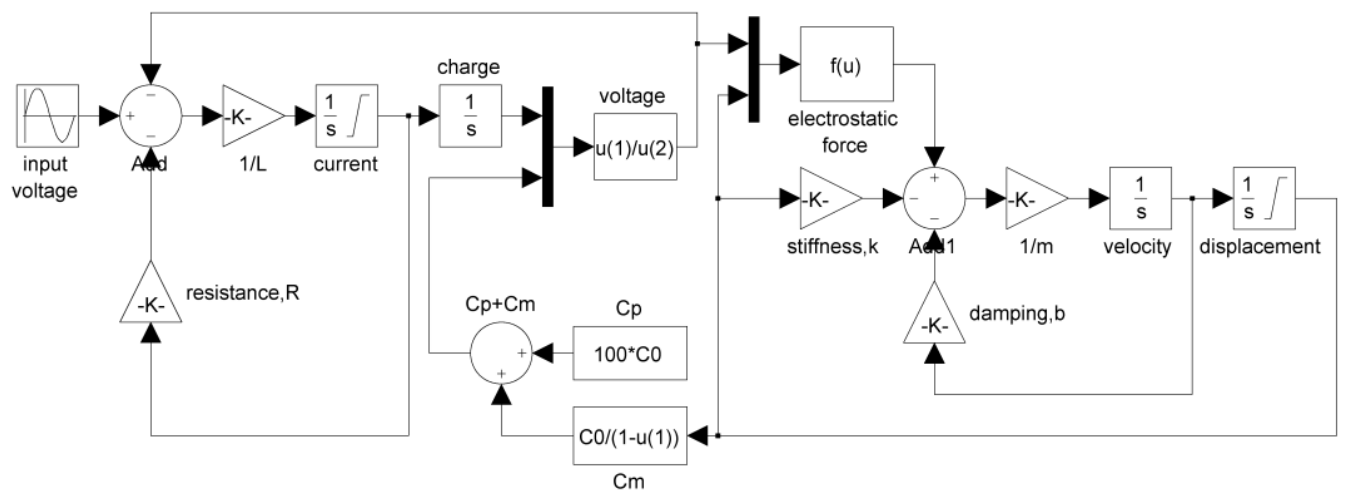

Figure 3.14 Block diagram of the numerical model of the parallel-plate actuator coupled with the resonant drive circuit that is created in Matlab/Simulink; its left side representing the resonant drive circuit and the right side depicting the parallel-plate actuator.

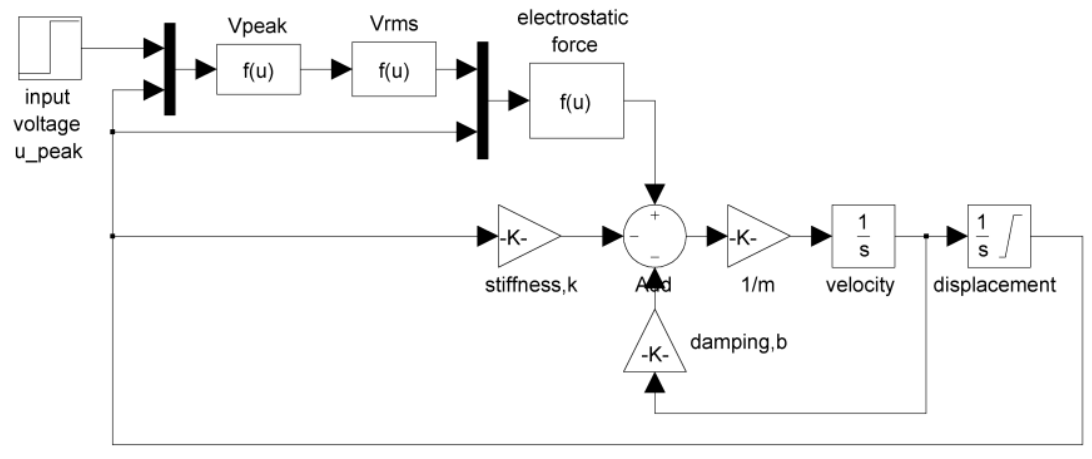

Figure 3.15 Block diagram of the numerical model of the simplified parallel-plate actuator coupled with the resonant drive circuit represented by Eq. 3.16.

The phase detector introduced in Section 3.5 is used to determine the displacement of the parallelplate actuator coupled with the resonant drive circuit, and the displacement measured by the phase detector is compared to the displacement obtained by the numerical integration in order to validate the proposed method. As presented in Figure 3.16, the measured displacement by the phase detector is in exact agreement with the displacement obtained by the numerical integration. In Figure 3.16, the solid gray lines represent the high frequency actuation voltage that is amplified by the resonant drive circuit and is obtained by the numerical model presented in Figure 3.14. The solid blue line, representing the envelope of the actuation voltage, is acquired from the simplified numerical mode in Figure 3.15. The normalized displacement denoted by the red solid line is attained from both numerical models through time integration. As presented, both displacements are in exact agreement to each other. Finally, the black dots represent the normalized displacements measured by the phase detector at the $100 \mathrm{kHz}$ sampling rate and are in good agreement with the displacement obtained by the time integration. 
The fixed points of the parallel-plate actuator coupled with the resonant drive circuit are also evaluated at various input voltages in order to compare them to the analytical results depicted in Figure 3.2, in which the black line represents the fixed points in the case of $\mathbf{Q}_{\mathrm{f}}=50$ and $\mathbf{r}=100$. We sweep the input voltage upward and downward in order to determine the fixed points while waiting for the transient response to decay. Its numerical simulation results are presented in Figure 3.17 and are in exact agreement with the analytical results in Figure 3.2. As we increase the input voltage to the resonant drive circuit, the saddle-node bifurcation occurs at $\overline{\mathbf{u}}=0.0296$ and $\mathbf{x}=0.401$, where the fixed point loses its stability, and the parallel-plate actuator jumps onto the stable fixed point in the upper branch represented by the blue solid line. If we reverse the voltage sweep, starting from the upper branch as the initial condition, the parallel-plate actuator does not undergo the saddle-node bifurcation at the previous bifurcation point. Its displacement decreases further down, until the another saddle-node bifurcation occurs, making the parallel-plate actuator jumping down onto the lower branch of the stable fixed points represented in the solid red line in Figure 3.17.

Table 3.1 Physical parameters used in the numerical model of the parallel-plate actuator coupled with the resonant drive circuit controlled by amplitude modulation

\begin{tabular}{llll}
\hline \hline Parameter & Symbol & Value \\
\hline Spring constant & $\mathrm{k}$ & $1[\mathrm{mN} / \mathrm{m}]$ \\
\hline Mass & $\mathrm{m}$ & $2 \mathrm{e}-10[\mathrm{~kg}]$ \\
\hline Area & $\mathrm{A}$ & $1 \mathrm{e}-8\left[\mathrm{~m}^{2}\right]$ \\
\hline Initial gap & $\mathrm{g}_{0}$ & $50[\mu \mathrm{m}]$ \\
\hline Permittivity of air & $\varepsilon$ & $8.85 \mathrm{e}-12[\mathrm{~F} / \mathrm{m}]$ \\
\hline Pull-in Voltage & $\mathrm{V}_{\mathrm{pi}}$ & $20.457[\mathrm{~V}]$ \\
\hline Damping coefficient & $\mathrm{b}$ & $8.944 \mathrm{e}-7[\mathrm{~N} \cdot \mathrm{s} / \mathrm{m}]$ \\
\hline Inductance & $\mathrm{L}$ & $10[\mathrm{mH}]$ \\
\hline Parasitic resistance & $\mathrm{R}$ & $4730[\Omega]$ \\
\hline Initial capacitance & $\mathrm{C}_{0}$ & $1.77[\mathrm{fF}]$ \\
\hline Parasitic capacitance & $\mathrm{C}_{\mathrm{p}}$ & $177[\mathrm{fF}]$ \\
\hline Ratio of $\mathrm{C}_{\mathrm{p}}$ to $\mathrm{C}_{0}$ & $\mathrm{r}$ & 100 \\
\hline Initial quality factor of the resonant drive circuit & $\mathrm{Q}_{\mathrm{f}}$ & 50 \\
\hline Natural frequency of the parallel-plate actuator & $\mathrm{f}_{\mathrm{m}}$ & $355.9[\mathrm{~Hz}]$ \\
\hline Natural frequency of the parallel-plate actuator & $\omega_{\mathrm{m}}$ & $2236[\mathrm{rad} / \mathrm{sec}]$ \\
\hline Initial resonant frequency of the resonant drive circuit & $\mathrm{f}_{0}$ & $3.764[\mathrm{MHz}]$ \\
\hline Initial resonant frequency of the resonant drive circuit & $\omega_{0}$ & $23.65 \mathrm{e} 6[\mathrm{rad} / \mathrm{sec}]$ \\
\hline \hline
\end{tabular}




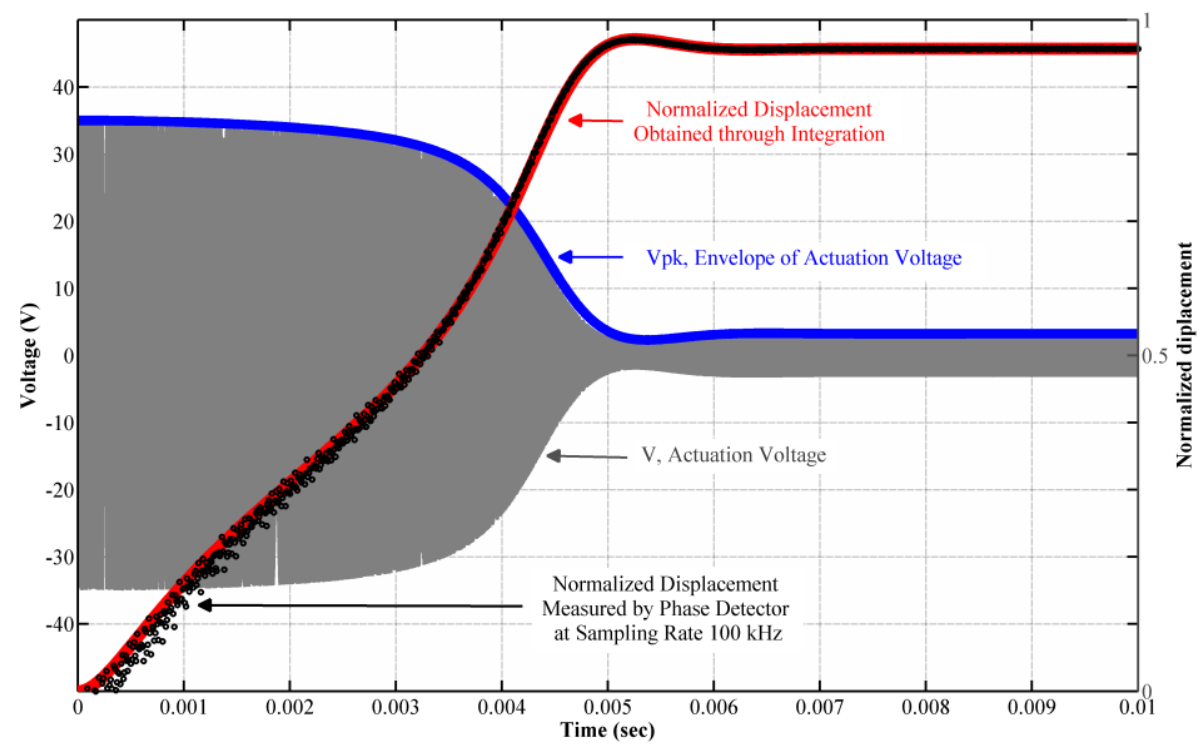

Figure 3.16 Step response of the parallel-plate actuator driven by the resonant drive circuit excited by the input voltage $\mathbf{u}=0.7 \mathrm{~V}$, which corresponds to the normalized input voltage $\overline{\mathbf{u}}=0.0342$; the actuation voltage is obtained from the numerical model in Figure 3.14; and the envelope of the actuation voltage is acquired from the numerical model in Figure 3.15.

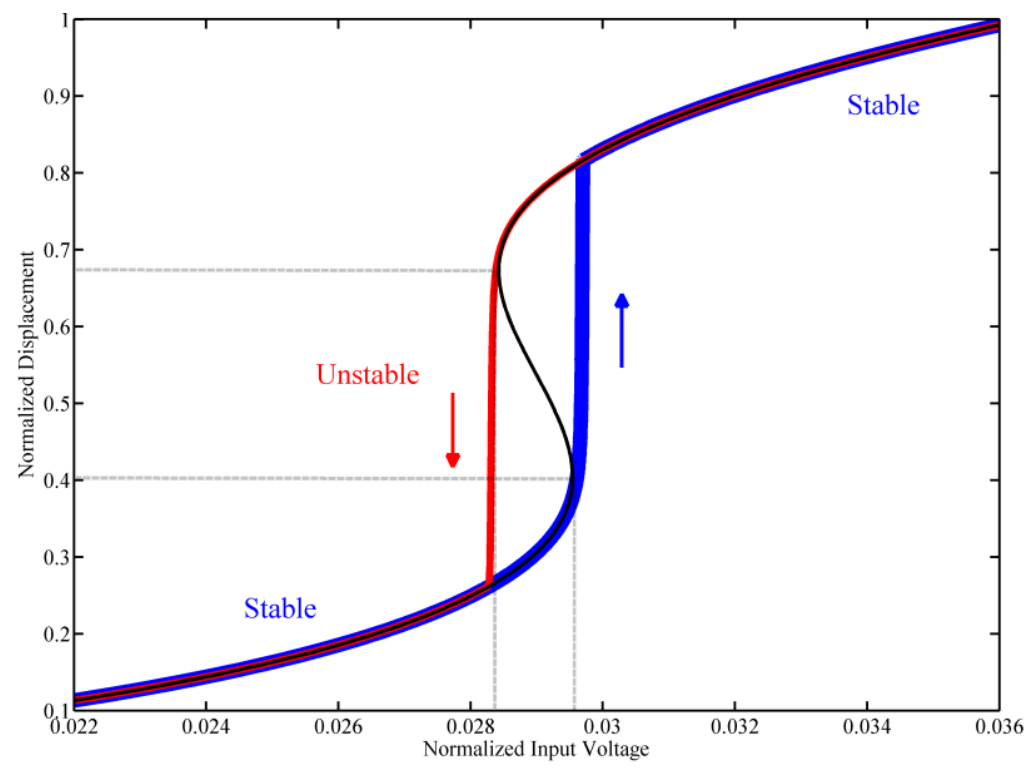

Figure 3.17 Hysteresis of the parallel-plate actuator driven by the resonant drive circuit in case of the initial quality factor, $\mathbf{Q}_{\mathrm{f}}=50$ and the ratio of $\mathbf{C}_{\mathbf{p}}$ to $\mathbf{C}_{\mathbf{0}}, \mathbf{r}=100$; the fixed points represented by the blue thick solid line are obtained by the upward voltage sweep; and the fixed points denoted by the red solid line are acquired by the downward voltage sweep. 


\subsection{Experiment on Parallel Plates}

In order to validate the analytical model of the parallel-plate actuator coupled with the resonant drive circuit and the feasibility of the proposed displacement measurement method, we build the parallelplate actuator on a macro scale, which consists of two single-sided copper clad boards with FR4 substrate. Their size is $50 \mathrm{~mm}$ in length, $50 \mathrm{~mm}$ in width, and $0.79 \mathrm{~mm}$ in thickness. One plate is mounted and fixed on the platform, while another plate is mounted on a micropositioner, which is used to adjust the gap, $1.2 \mathrm{~mm}$, between two parallel plates, as presented in Figure 3.18-(b).

We measure the capacitance of the parallel plates using the LCR meter, 889A from BK Precision, to determine the parasitic capacitance. We build the resonant drive circuit with the $3.3 \mathrm{mH}$ ferrite-core inductor, 22R335C from Murata Power Solutions, and the current-sensing transformer, 53050C from the same company, to convert the current through the resonant drive circuit to the voltage. The additional discrete capacitors, $500 \mathrm{pF}$ and $1000 \mathrm{pF}$, are used to vary the parasitic capacitance so that we can determine how the parasitic capacitance affects the voltage gain and the phase delay of the resonant drive circuit. The function generator 4086 from BK Precision is used as the AC input voltage source to excite the resonant drive circuit.

Based on the initial capacitance of the parallel-plate actuator, its measured parasitic capacitance, and the inductance of the inductor in the resonant drive circuit, we estimate the initial electrical resonant frequency $\boldsymbol{\omega}_{\mathbf{0}}$. Then, using the function generator and the estimated resonant frequency, we excite the resonant drive circuit with a low voltage sine wave, $1 \mathrm{~V}_{\mathrm{pp}}$, while sweeping the frequency of the input signal in order to determine the precise electrical resonant frequency at which the phase delay of the actuation voltage is $90 \mathrm{deg}$ with reference to the input voltage and the voltage gain is maximum.

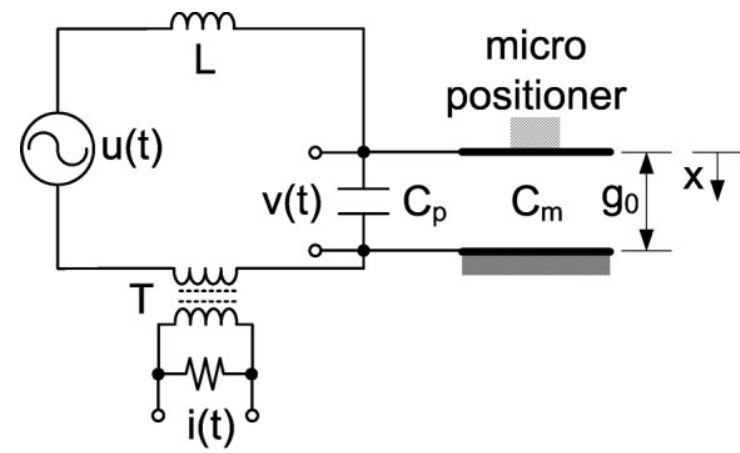

(a)

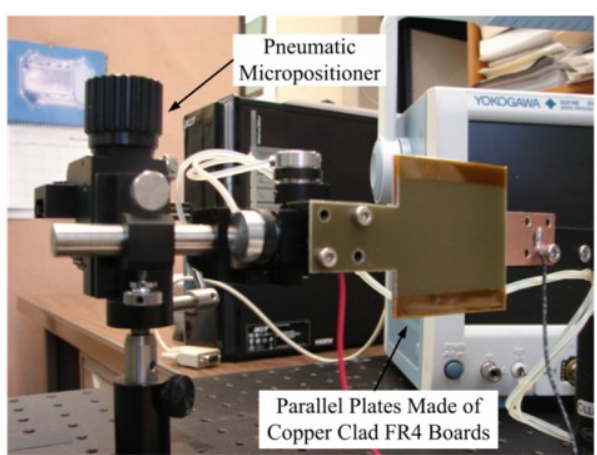

(b)

Figure 3.18 (a) Schematic of the experiment set-up with the parallel-plates controlled by the micropositioner and (b) the picture showing the parallel-plates with the micropositioner

After determining the initial electrical resonance, we quantify the initial quality factor $\mathbf{Q}_{\mathbf{f}}$ and the parasitic resistance $\mathbf{R}$ of the resonant drive circuit. While varying the gap between two parallel plates, we measure the input voltage, the actuation voltage, and the current through the resonant drive circuit to determine the voltage gain, the current gain, the phase delay of the actuation voltage, and the phase delay of the current with respect to the input voltage. 
The voltage gain and the phase delay between the input voltage and the actuation voltage are presented in Figure 3.19-(a) and (b), respectively. The measurements denoted by solid squares $\left(\mathbf{C}_{\mathbf{p}}=515 \mathrm{pF}\right)$ and solid circles $\left(\mathbf{C}_{\mathbf{p}}=1015 \mathrm{pF}\right)$ agree well with the analytical results represented by the red solid lines and the blue dashed lines in Figure 3.19. The current gain and the phase delay between the input voltage and the input current are presented in Figure 3.20-(a) and (b), respectively. The measurements denoted by solid squares $\left(\mathbf{C}_{\mathbf{p}}=515 \mathrm{pF}\right)$ and solid circles $\left(\mathbf{C}_{\mathbf{p}}=1015 \mathrm{pF}\right)$ are also in good agreement with the analytical results represented by the solid line and the dashed line in Figure 3.20.

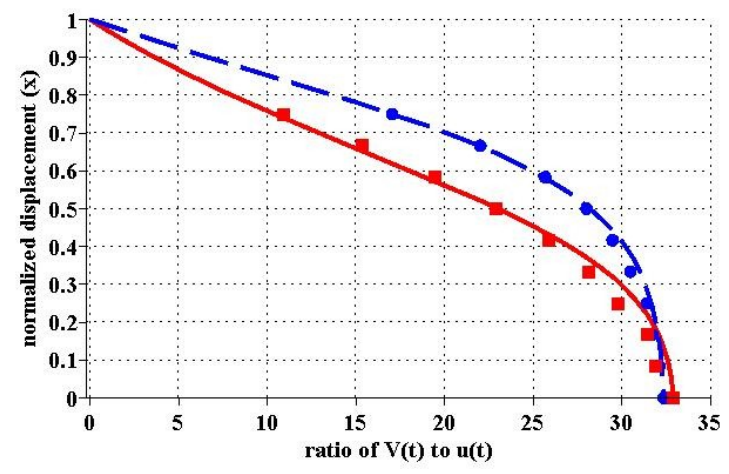

(a)

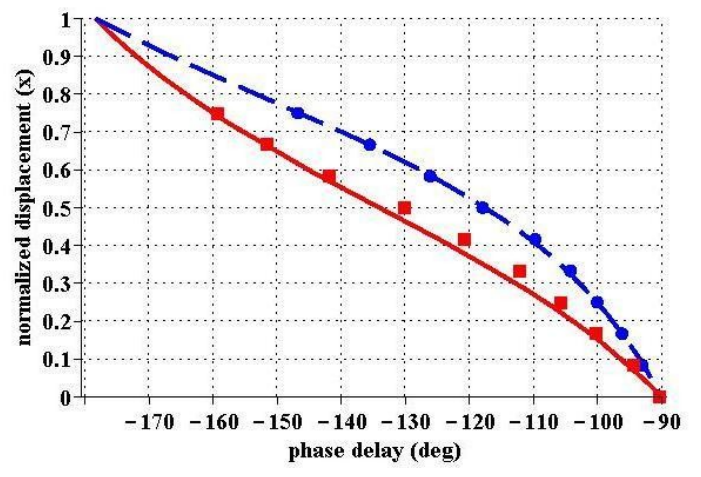

(b)

Figure 3.19 Displacement measurement of the parallel-plates through (a) the voltage gain and (b) the phase delay of the actuation voltage with respect to the input voltage: the red solid line represents the analytical result, and the red solid boxes denote the measurement with $\mathbf{C}_{\mathbf{p}}=515 \mathrm{pF}$; the blue dashed line represents the analytical result, and the blue solid circles denote the measurement with $\mathbf{C}_{\mathbf{p}}=1015 \mathrm{pF}$.

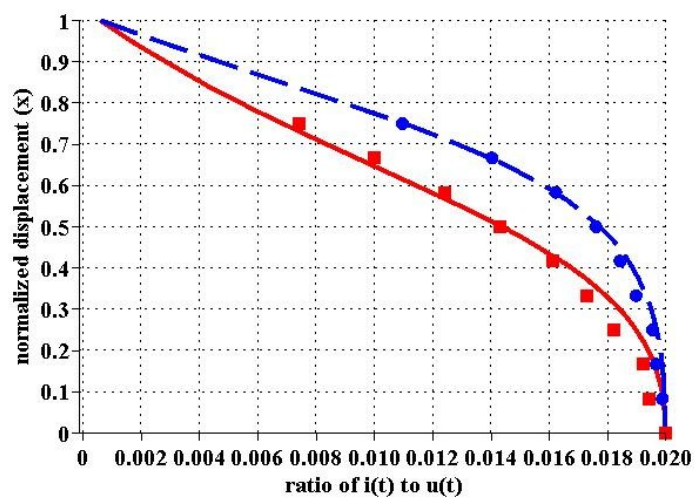

(a)

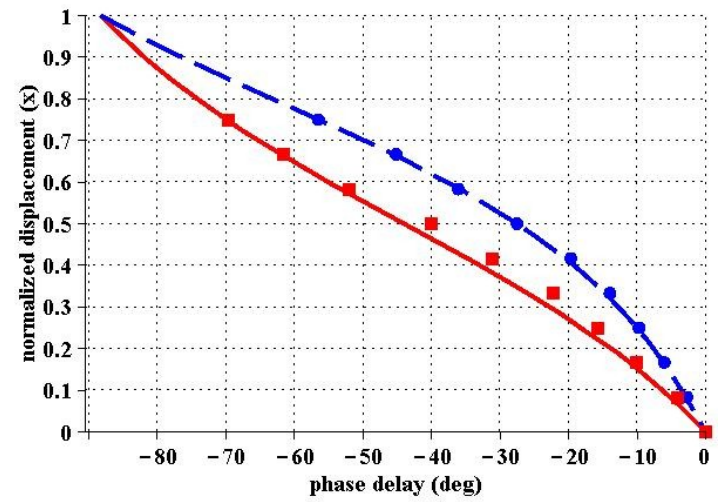

(b)

Figure 3.20 Displacement measurement of the parallel-plates through the current gain (a) and phase delay of the current with respect to the input voltage (b); the red solid line representing the estimation and the red solid boxes denoting the measurement with $\mathbf{C}_{\mathbf{p}}=515 \mathrm{pF}$; the blue dashed line representing the estimation and the blue solid circles representing the measurement with $\mathbf{C}_{\mathbf{p}}=1015 \mathrm{pF}$. 
While measuring the gain and the phase delay, we capture the screen shots of the oscilloscope as presented in Figure 3.21 and Figure 3.22, where the input voltage, the actuation voltage, and the current are shown in the time domain. Figure 3.21 and Figure 3.22 also present the Lissajous patterns representing the voltage gain, the current gain, and the phase delay with reference to the input voltage in the cases of the parasitic capacitance $\mathbf{C}_{\mathbf{p}}=1015 \mathrm{pF}$ and $\mathbf{C}_{\mathbf{p}}=515 \mathrm{pF}$, respectively.

In both Figure 3.21 and Figure 3.22, the input voltage is represented by the yellow solid lines as Ch 1, the actuation voltage is denoted by the solid green lines as $\mathrm{Ch} 2$, and the input current is represented by the solid light blue lines as $\mathrm{Ch} 4$. The scale of $\mathrm{Ch} 1$ is set to be $500 \mathrm{mV} / \mathrm{div}$, which corresponds to the input voltage of $1 \mathrm{~V}_{\mathrm{pp}}$. The scale of $\mathrm{Ch} 2$ is set to be $5 \mathrm{~V} / \mathrm{div}$, and the scale of $\mathrm{Ch} 3$ is set to be $20 \mathrm{mV} / \mathrm{div}$.

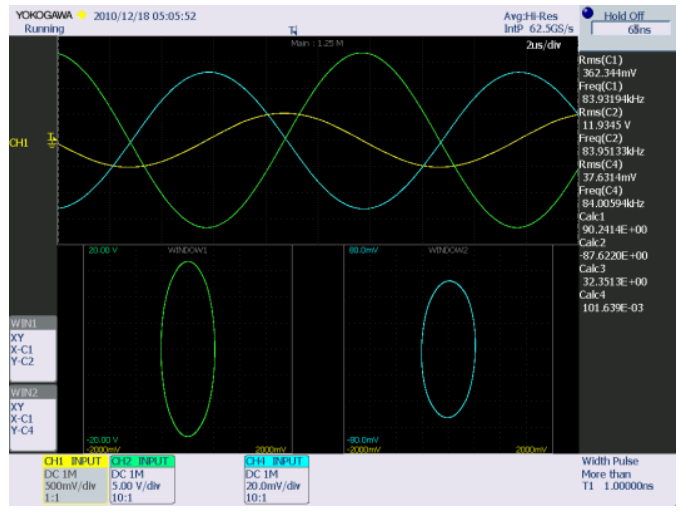

(a)

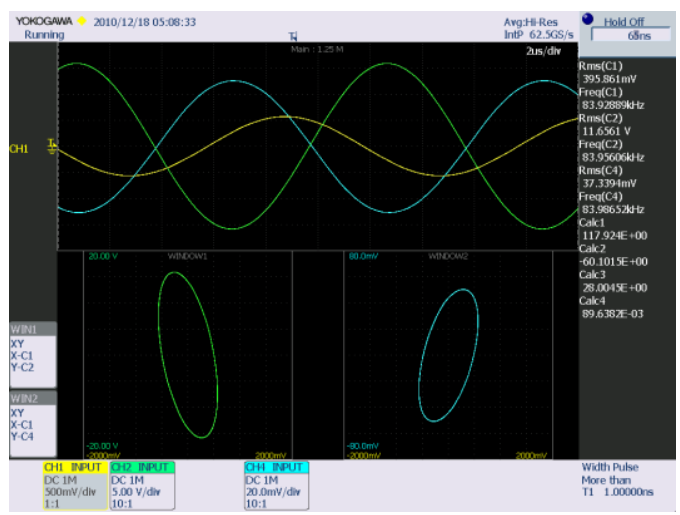

(c)

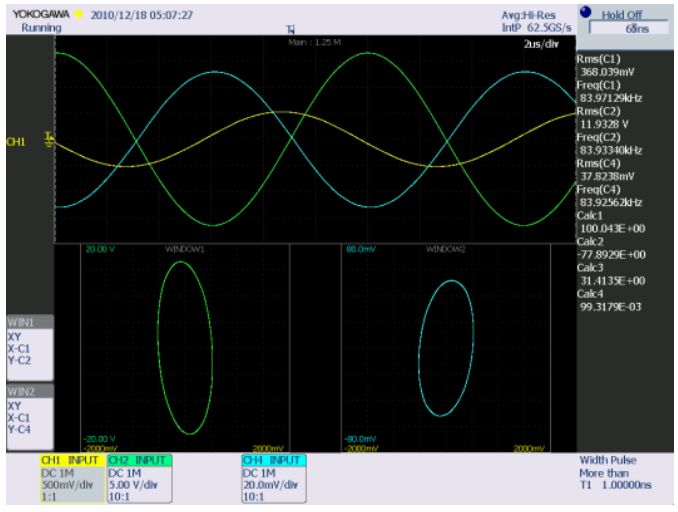

(b)

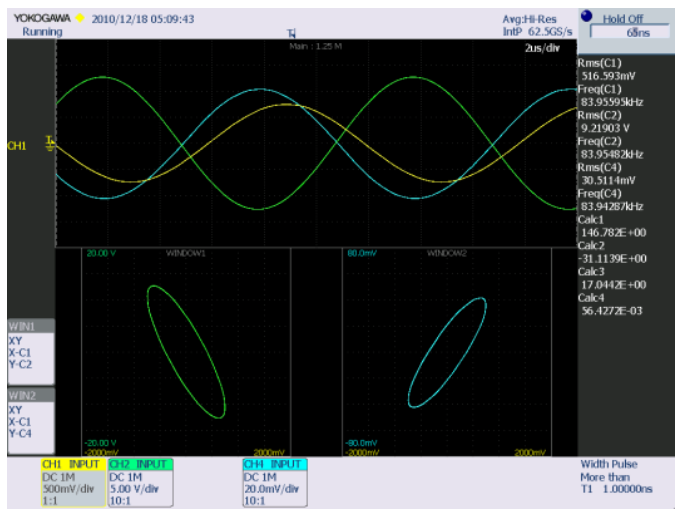

(d)

Figure 3.21 Screenshots of the oscilloscope that capture the input voltage (Ch 1, Yellow), the actuation voltage (Ch 2, Green), the current (Ch 4, Light Blue) through the resonant drive circuit in the main window, the Lissajous pattern of $\mathrm{Ch} 1$ and $\mathrm{Ch} 2$ in the $1^{\text {st }}$ window and the Lissajous pattern of $\mathrm{Ch} 1$ and $\mathrm{Ch} 4$ in the $2^{\text {nd }}$ window with $\mathbf{Q}_{\mathrm{f}}=32.35$ and $\mathbf{r}=55$ at (a) zero displacement, (b) $300 \mu \mathrm{m}$ displacement, (c) $600 \mu \mathrm{m}$ displacement, and (d) $900 \mu \mathrm{m}$ displacement 
Because it is difficult to see how each signal is related to each other in terms of the amplitude and the phase, we make use of the Lissajous curves and present them in the $2^{\text {nd }}$ and $3^{\text {rd }}$ window in each screenshot. The $2^{\text {nd }}$ window presents the Lissajous curve, in which the $\mathrm{x}$ axis is represented by the input voltage $\mathrm{Ch} 1$ and the $\mathrm{y}$ axis is denoted by the actuation voltage $\mathrm{Ch} 2$. The $3^{\text {rd }}$ window presents the Lissajous curve, in which the $\mathrm{x}$ axis is represented by the same input voltage Ch 1 and the $\mathrm{y}$ axis is denoted by the input current $\mathrm{Ch} 4$. In both windows, the eccentricity of the Lissajous ellipse represents the ratio of the amplitude of the actuation voltage or the input current to the amplitude of the input signal. Moreover, the angle between its major axis and the $\mathrm{x}$ axis represents the phase difference between two signals.

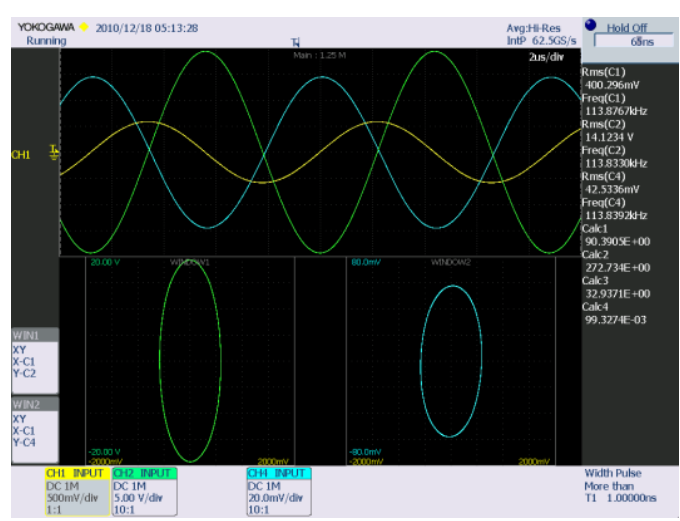

(a)

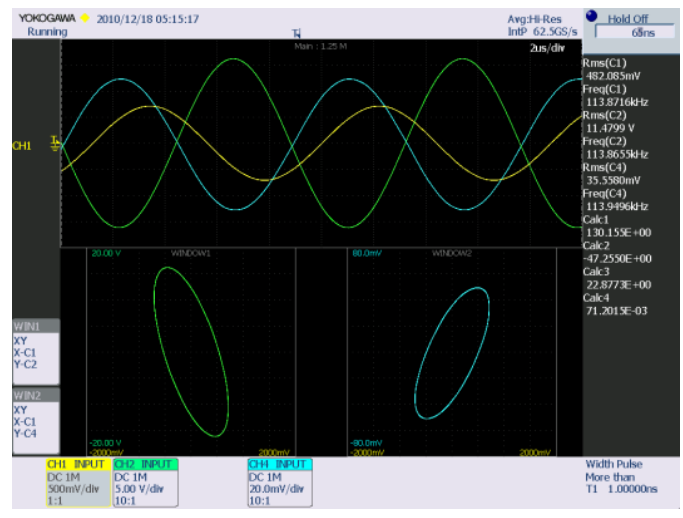

(c)

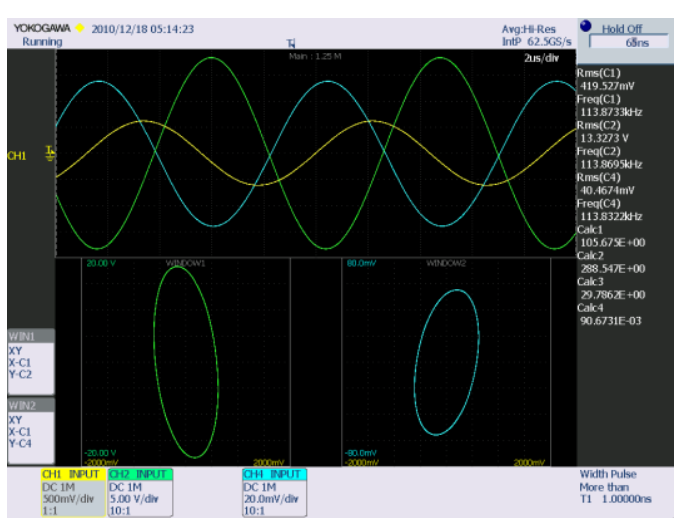

(b)

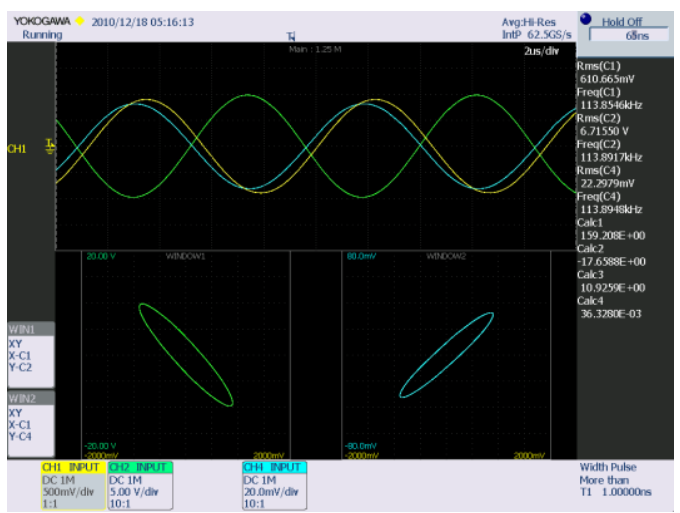

(d)

Figure 3.22 Screenshots of the oscilloscope that capture the input voltage (Ch 1, Yellow), the actuation voltage (Ch 2, Green), the current (Ch 4, Light Blue) through the resonant drive circuit in the main window, the Lissajous pattern of $\mathrm{Ch} 1$ and $\mathrm{Ch} 2$ in the $1^{\text {st }}$ window and the Lissajous pattern of Ch 1 and Ch 4 in the $2^{\text {nd }}$ window with $\mathbf{Q}_{\mathrm{f}}=32.94$ and $\mathbf{r}=28$ at (a) zero displacement, (b) $300 \mu \mathrm{m}$ displacement, (c) $600 \mu \mathrm{m}$ displacement, and (d) $900 \mu \mathrm{m}$ displacement 


\subsection{Summary}

In this chapter, we derive and present the analytical model of the parallel-plate actuator coupled with the resonant drive circuit. Using this analytical model, we evaluate the nonlinear behavior of the parallel-plate actuator with various parasitic capacitances and the quality factor of the resonant drive circuit in Section 3.1 and Section 3.2. The analytical results show that the resonant drive circuit is not only capable of driving the parallel-plate actuator at the lower input voltage, but is also able to extend the operation range of the parallel-plate actuator beyond the conventional pull-in point in the presence of high parasitic capacitance. Furthermore, the hysteresis in its fixed points, which is never been reported, is theoretically predicted and confirmed by its numerical model in Section 3.6. The nonlinear dynamics of the parallel-plate actuator coupled with the resonant drive circuit is also studied in Section 3.3 and Section 3.4, when the resonant drive circuit is excited by the input signal modulated by the frequency modulation. In Section 3.5, we introduce the new displacement measurement method for electrostatic actuators. By measuring the phase delay between the actuation voltage and the input voltage, we are able to determine the displacement of the parallel-plate actuator, and the experiment results described in Section 3.7 are in good agreement with the analytical results. This new displacement measurement method is used to implement the feedback control of the parallel-plate actuator described in Chapter 7. 


\section{Chapter 4 \\ Implementation of Resonant Drive Circuit}

The resonant drive circuit introduced in Chapter 3 can be implemented in various ways: to name a few, an unbalanced resonant drive circuit and a balanced resonant drive circuit. In this chapter, we discuss how to implement the resonant drive circuit with discrete electrical components and present a set of the resonant drive circuits in order to characterize their performance in terms of the voltage gain, the quality factor, and the robustness to the saddle-node bifurcation.

This chapter is organized as follows. In Section 4.1, a simple but effective way to control an unbalanced resonant drive circuit is to pair the unbalanced resonant drive circuit with a unity gain amplifier, which acts as a buffer to provide low output impedance. In Section, 4.2, a couple of the balanced resonant drive circuits are presented. An analytical model of the parallel-plate actuator coupled with the balanced resonant drive circuit is derived in order to compare the unbalanced and balanced resonant drive circuit in terms of their voltage gain and quality factor. The same multiple scale method [43] described in Chapter 3 is used to transform a linear differential equation of the balanced resonant drive circuit into the algebraic equation through a frequency response. The fixed points of the parallel-plate actuator coupled with the unbalanced and balanced resonant drive circuits are evaluated as well, and their stability is also analyzed and presented in Section 4.2. In Section 4.3, we introduce a new way of enhancing the inherent negative feedback of the unbalanced resonant drive circuit by putting the unbalanced resonant drive circuit into a positive feedback loop of a noninverting amplifier, the voltage gain of which decreases as the displacement of the parallel-plate actuator increases. Because the increase of the displacement of the parallel-plate actuator leads into the increase of its capacitance, which, in turn, raises the impedance of the resonant drive circuit, this increase in the impedance of the resonant drive circuit reduces the amount of the positive feedback, resulting in a lower voltage gain. As a result, the parallel-plate actuator is stabilized beyond its conventional pull-in point. To evaluate its fixed points and stability, we derive and present its analytical model. Furthermore, we present the circuit simulation results of the non-inverting amplifier implemented with both negative and positive feedback loops: the negative feedback loop defines the minimum gain of the amplifier and its positive feedback loop provides an additional voltage gain that decrease by the increase in the impedance of the resonant drive circuit, which is part of the positive feedback loop.

\subsection{Unbalanced Resonant Drive Circuit}

In this section, we present how an unbalanced resonant drive circuit is implemented with discrete electrical components. Its governing equation is the same as that presented in Chapter 3. A simple but effective way to implement a dynamic voltage control, including amplitude modulation and frequency modulation is an op-amp used to excite the resonant drive circuit presented in Figure 4.1. An electrostatic actuator is represented by the variable capacitor $\mathbf{C}_{\mathbf{m}}$, the parasitic capacitance of which is denoted by $\mathbf{C}_{\mathbf{p}}$ in Figure 4.1. The resonant drive circuit is driven by an op-amp that is configured as a unity-gain amplifier, also known as a voltage follower that provides low output impedance. 


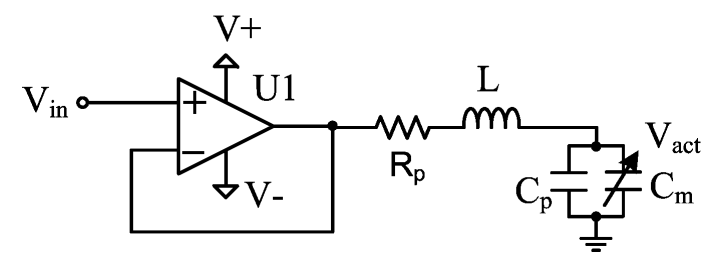

Figure 4.1 Electrostatic actuator, represented by $\mathbf{C}_{\mathbf{m}}$, coupled with the unbalanced resonant drive circuit that is driven by a unity-gain amplifier U1, also known as a buffer.

Suppose that the electrostatic actuator represented by $\mathbf{C}_{\mathbf{m}}$ is the parallel-plate actuator, the electrostatic actuator in Figure 4.1 has the same governing equations depicted in Eq. 3.1, Eq. 3.2 and Eq. 3.3. Needless to say, it exhibits the same responses presented in Figure 3.2 in terms of the parasitic capacitance and the quality factor of the resonant drive circuit.

\subsection{Balanced Resonant Drive Circuits}

A simplified schematic of the balanced resonant drive circuit is presented in Figure 4.2, where two voltage sources are used to drive the balanced resonant drive circuit, the governing equation of which is shown in Eq. 4.1.

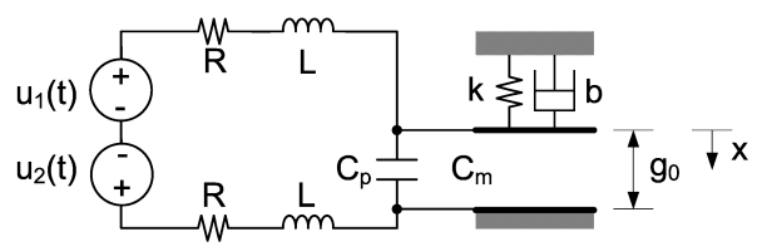

Figure 4.2 the parallel-plate actuator coupled with the balanced resonant drive circuit driven by two voltage sources $180^{\circ}$ out of phase to each other.

Obviously, the governing equation of the parallel-plate actuator is the same as Eq. 3.1 in the previous chapter. The only difference between the unbalanced and balanced resonant drive circuit resides in the electrical circuits: two voltage sources, which are $180^{\circ}$ out of phase to each other, are implemented to drive the balanced resonant drive circuit.

$$
2 L \ddot{q}+2 R \dot{q}+\frac{1}{C_{p}+C_{m}} q=2 u
$$

where $\mathbf{L}$ represents the inductance of the inductor; $\mathbf{R}$ denotes the parasitic resistance of the resonant drive circuit and the parallel-plate actuator that is represented by $\mathbf{C}_{\mathbf{m}}$; the parasitic capacitance of the electrostatic actuator is denoted by $\mathbf{C}_{\mathbf{p}}$; $\mathbf{q}$ represents the charge stored in both the parallel-plate actuator and its parasitic capacitance; and $\mathbf{u}$ denotes the peak amplitude of the input voltage.

Using the same multiple scale method [43], we are able to determine the voltage gain that is the function of the capacitance $\mathbf{C}_{\mathbf{m}}$ of the parallel-plate actuator, which is considered as a variable on a 
slow time scale. The voltage gain obtained through the Laplace transform is presented in Eq. 4.2 as thus:

$$
H(s)=\frac{V(s)}{U(s)}=\frac{2}{2 L C s^{2}+2 R C s+1}=\frac{\frac{1}{L\left(C_{p}+C_{m}\right)}}{s^{2}+\frac{R}{L} s+\frac{1}{2 L\left(C_{p}+C_{m}\right)}}
$$

where $\mathbf{U}$ and $\mathbf{V}$ represent the input voltage and the actuation voltage across an electrostatic actuator in the frequency domain, respectively; $\mathbf{C}$ represents total capacitance of an electrostatic actuator, the sum of $\mathbf{C}_{\mathbf{m}}$ and $\mathbf{C}_{\mathbf{p}}$. In order to derive the voltage gain, we evaluate the frequency response of the resonant drive circuit, where $\mathbf{C}_{\mathbf{0}}, \boldsymbol{\omega}_{\mathbf{0}}, \mathbf{r}$, and $\mathbf{Q}_{\mathbf{f}}$ are the initial capacitance of an electrostatic actuator, the frequency of the input signal that is fixed at the initial resonant frequency of the resonant drive circuit, the ratio of the parasitic capacitance $\mathbf{C}_{\mathbf{p}}$ to the initial capacitance of an electrostatic actuator $\mathbf{C}_{\mathbf{0}}$, and the initial quality factor of the resonant drive circuit, respectively.

In order to determine the difference in the voltage gain and the phase change of the unbalanced and balanced resonant drive circuit implemented with the same discrete electronic components, we derive and present the frequency responses of both resonant drive circuits in Figure 4.3.
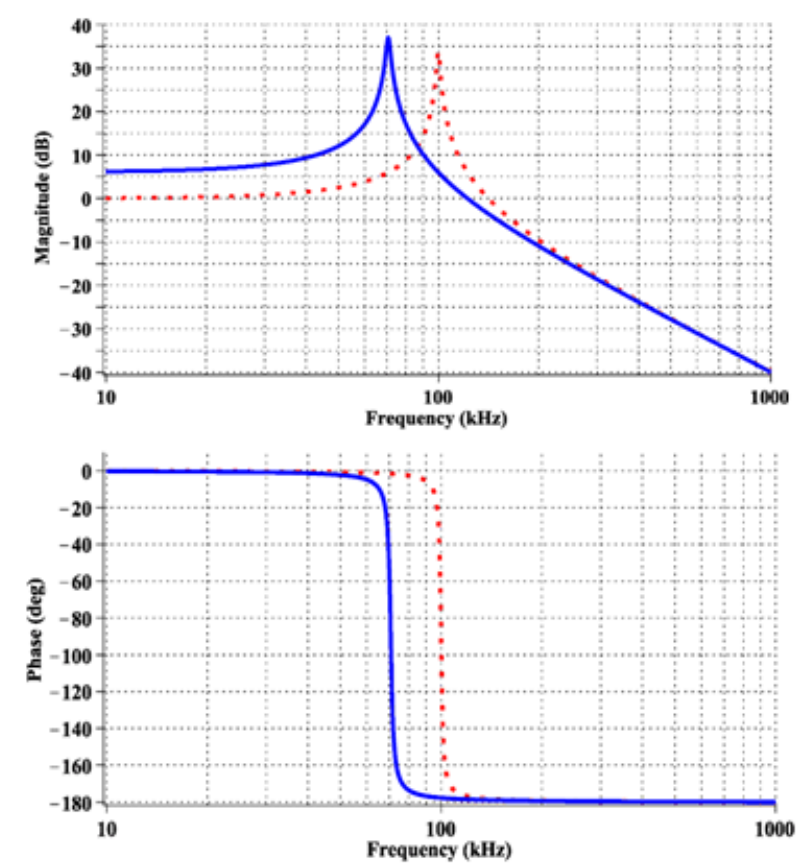

Figure 4.3 Frequency responses of the unbalanced (the red dotted lines) and the balanced resonant drive circuits (the blue solid lines) implemented with the same electronic components.

In Figure 4.3, the frequency response of the unbalanced resonant drive circuit is represented by the red dotted lines, and the frequency response of the balanced resonant drive circuit is depicted by the blue solid lines. The quality factor of the unbalanced resonant drive circuit is set to be $50(34 \mathrm{~dB})$, and its resonant frequency is set to be $100 \mathrm{kHz}$. The quality factor of the balanced resonant drive circuit 
implemented with the same electronic components is $35.4(31 \mathrm{~dB})$, and its resonant frequency is $70.7 \mathrm{kHz}$. However, the voltage gain of the balanced resonant drive circuit is $\sqrt{2}$ times higher than that of the unbalanced resonant drive circuit, because the amplitude of the input voltage to the balanced resonant drive circuit is twice higher than that of the unbalanced resonant drive circuit, and the quality factor of the unbalanced resonant drive circuit is $\sqrt{2}$ times higher than that of the balanced resonant drive circuit. Hence, in the end, the voltage gain of the balanced resonant drive circuit is 70.7, which is equal to $37 \mathrm{~dB}$, as presented in Figure 4.3.

In order to simplify the derivation of the governing equation of electrostatic actuators coupled with the balanced resonant drive circuit, we define the initial resonant frequency, the ratio of the parasitic capacitance to the initial capacitance of the electrostatic actuators, and the quality factor of the balanced resonant drive circuit as thus:

$$
\omega_{0}=\frac{1}{\sqrt{2 L C_{0}(r+1)}}, \quad r=\frac{C_{p}}{C_{0}}, \quad Q_{f}=\sqrt{\frac{L}{2 R^{2} C_{0}(r+1)}}
$$

Suppose that the frequency of the input voltage source is equal to the initial resonant frequency of the resonant drive circuit, remains constant and is much higher than the mechanical resonant frequency of the electrostatic actuator, we evaluate the voltage gain of the balanced resonant drive circuit in terms of the initial quality factor $\mathbf{Q}_{\mathrm{f}}$, the parasitic capacitance $\mathbf{C}_{\mathbf{p}}$, and the capacitance of an electrostatic actuator $\mathbf{C}_{\mathbf{m}}$ through the frequency response as thus:

$$
\begin{aligned}
|H(j \omega)|_{\omega=\omega_{0}} & =\frac{2}{\sqrt{\left(1-\frac{C_{p}+C_{m}}{C_{0}(r+1)}\right)^{2}+\left(\frac{2 R\left(C_{p}+C_{m}\right)}{\left.\sqrt{2 L C_{0}(r+1)}\right)^{2}}\right.}} \\
& =\frac{2}{\sqrt{\left(\frac{C_{0}-C_{m}}{C_{0}(r+1)}\right)^{2}+\frac{2 R^{2}\left(C_{p}+C_{m}\right)^{2}}{L C_{0}(r+1)}}} \\
& =\frac{2 Q_{f}(r+1)}{\sqrt{Q_{f}{ }^{2}\left(1-\frac{C_{m}}{C_{0}}\right)^{2}+\left(r+\frac{C_{m}}{C_{0}}\right)^{2}}} \\
& =\frac{2 Q_{f} C_{0}(r+1)}{\sqrt{Q_{f}{ }^{2}\left(C_{0}-C_{m}\right)^{2}+\left(C_{p}+C_{m}\right)^{2}}}
\end{aligned}
$$

Suppose that the electrostatic actuator is the same parallel-plate actuator presented in Chapter 3, and the fringe field of the parallel-plate actuator is negligible, its capacitance $\mathbf{C}_{\mathbf{m}}$ has a following algebraic expression in terms of its normalized displacement $\mathbf{x}$ as thus:

$$
C_{m}=\frac{\varepsilon A}{g_{0}-X}=\frac{\varepsilon A}{g_{0}(1-x)}=\frac{C_{0}}{1-x}
$$


Using Eq. 4.5, we further simplify Eq. 4.4 into Eq. 4.6 representing the voltage gain of the balanced resonant drive circuit in terms of the normalized displacement of the parallel-plate actuator:

$$
\begin{aligned}
\left|H\left(j \omega_{0}\right)\right| & =\frac{2 Q_{f} C_{0}(r+1)}{\sqrt{Q_{f}^{2}\left(C_{0}-\frac{C_{0}}{1-x}\right)^{2}+C_{0}{ }^{2}\left(r+\frac{1}{1-x}\right)^{2}}} \\
& =\frac{2 Q_{f}(r+1)}{\sqrt{Q_{f}^{2}\left(\frac{x}{1-x}\right)^{2}+\left(\frac{r(1-x)+1}{1-x}\right)^{2}}} \\
& =\frac{2 Q_{f}(r+1)(1-x)}{\sqrt{Q_{f}{ }^{2} x^{2}+(r(1-x)+1)^{2}}}
\end{aligned}
$$

Since the parallel-plate actuator is too sluggish to follow the high frequency actuation voltage amplified by the balanced resonant drive circuit, it only responds to the RMS value of the actuation voltage. Using the voltage gain in Eq. 4.6, we derive the RMS value of the actuation voltage as thus:

$$
V_{r m s}=\frac{1}{\sqrt{2}}\left|H\left(j \omega_{0}\right)\right| u=\frac{\sqrt{2} Q_{f}(r+1)(1-x) u}{\sqrt{Q_{f}^{2} x^{2}+(r(1-x)+1)^{2}}}
$$

Substituting the actuation voltage $\mathbf{V}$ in Eq. 3.4 with $\mathbf{V}_{\text {rms }}$ in Eq. 4.7, we derive the governing equation of the parallel-plate actuator coupled with the balanced resonant drive circuit in terms of the normalized displacement as thus:

$$
m \ddot{x}+b \dot{x}+k x=\frac{\varepsilon A}{g_{0}{ }^{3}} \frac{Q_{f}{ }^{2}(r+1)^{2} u^{2}}{\left(Q_{f}^{2} x^{2}+(r(1-x)+1)^{2}\right)}
$$

Normalizing Eq. 4.8 with the conventional pull-in voltage [9] of the parallel-plate actuator in Eq. 4.9, we further simplify Eq. 4.8 into the non-dimensional governing equation in Eq. 4.10:

$$
\begin{gathered}
u=V_{\text {pull }-i n} \bar{u}=\sqrt{\frac{8 k g_{0}{ }^{3}}{27 \varepsilon A} \bar{u}} \\
\ddot{x}+2 \xi \omega_{n} \dot{x}+\omega_{m}{ }^{2} x=\frac{8}{27} \omega_{m}{ }^{2} \frac{Q_{f}{ }^{2}(r+1)^{2} \bar{u}^{2}}{\left(Q_{f}^{2} x^{2}+(r(1-x)+1)^{2}\right)}
\end{gathered}
$$

where $\overline{\mathbf{u}}$ denotes the normalized input voltage with respect to the conventional pull-in voltage of the parallel-plate actuator; $\xi$ represents the damping ratio of the parallel-plate actuator; $\boldsymbol{\omega}_{\mathbf{m}}$ stands for the mechanical natural frequency of the parallel-plate actuator.

In order to determine the operation range of the parallel-plate actuator coupled with both resonant drive circuits, we present the locations of the saddle-node bifurcation of the parallel-plate actuator in terms of its parasitic capacitance in Figure 4.4, where the black dotted line represents the saddle 
nodes of the parallel-plate actuator driven by charge control, the red solid line denote the saddle node of the unbalanced resonant drive circuits, and the blue dashed line represents the saddle node of the balanced resonant drive circuit implemented with the same components.

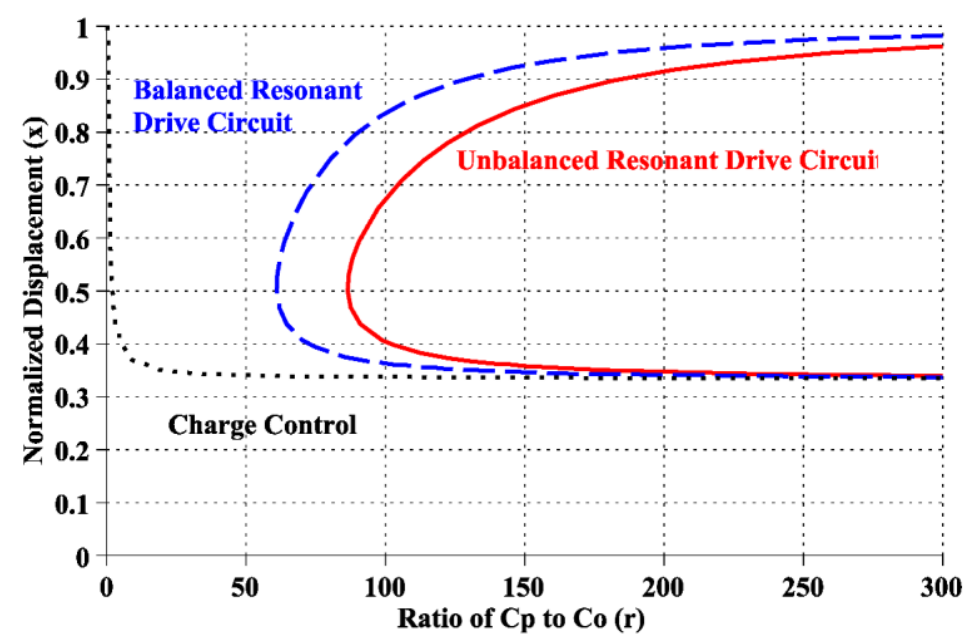

Figure 4.4 Saddle nodes of the parallel-plate actuator driven by charge control circuit, the unbalanced and balanced resonant drive circuits with the same components: in terms of the ratio of $\mathrm{C}_{\mathrm{p}}$ to $\mathrm{C}_{0}$

As presented in Figure 4.3 and Figure 4.4, the balanced resonant drive circuit has less robustness to the parasitic capacitance than the unbalanced resonant drive circuit, in return for the higher voltage gain.

\subsubsection{Op-Amps in the BTL Configuration}

The first balanced resonant drive circuit introduced in this chapter is implemented with two op-amps arranged in the bridge-tied load configuration, as presented in Figure 4.5, where U1 is configured as a non-inverting amplifier, and U2 is configured as an inverting amplifier with the voltage gain of 1 to provide $180^{\circ}$ phase shift. This balanced resonant drive circuit can be controlled by either amplitude modulation or frequency modulation without any modification.

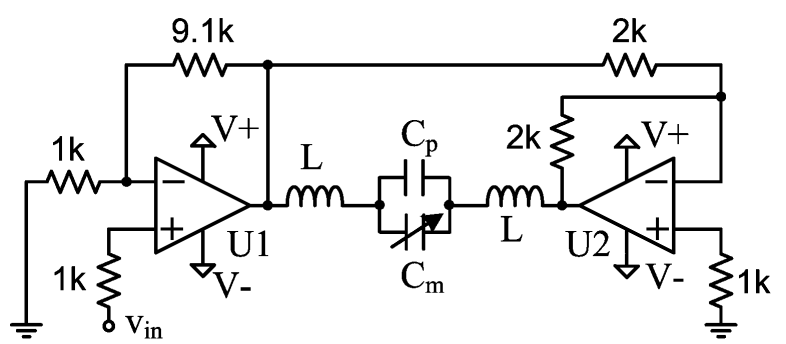

Figure 4.5 Balanced resonant drive circuit excited by two op-amps in the BTL configuration: U1 is configured as a non-inverting amplifier with the voltage gain of 10.1, and $\mathrm{U} 2$ is arranged to be an inverting buffer to provide $180^{\circ}$ phase shift with respect to the output of $\mathrm{U} 1$. 
The dynamics of the parallel-plate actuator coupled with this balanced resonant drive circuit is characterized by the same governing equation in Eq. 4.10.

\subsubsection{H-Bridge with MOSFET Drivers}

So far, we have been using an $\mathrm{AC}$ voltage source to excite the unbalanced resonant drive circuit and two AC voltage sources to control the balanced resonant drive circuit. In practice, each voltage source requires two complementary power supplies to provide a positive and a negative power rail, which requires, for example, a charge pump or a negative voltage regulator to supply a negative power rail from a positive power rail. This adds more electronic components into a control circuit. Hence, we design the resonant drive circuit that requires neither an $\mathrm{AC}$ voltage source nor a negative power rail. The balanced resonant drive circuit implemented with an H-bridge and two complementary MOSFET drivers presented in Figure 4.6 can be controlled by either amplitude modulation or frequency modulation. When amplitude modulation is selected to control the balanced resonant drive circuit in Figure 4.6, the applied voltage to the H-bridge shall be adjusted by a voltage regulator. When frequency modulation is chosen to control the same circuit, only the frequency of the input signal to the MOSFET drivers needs to be controlled.

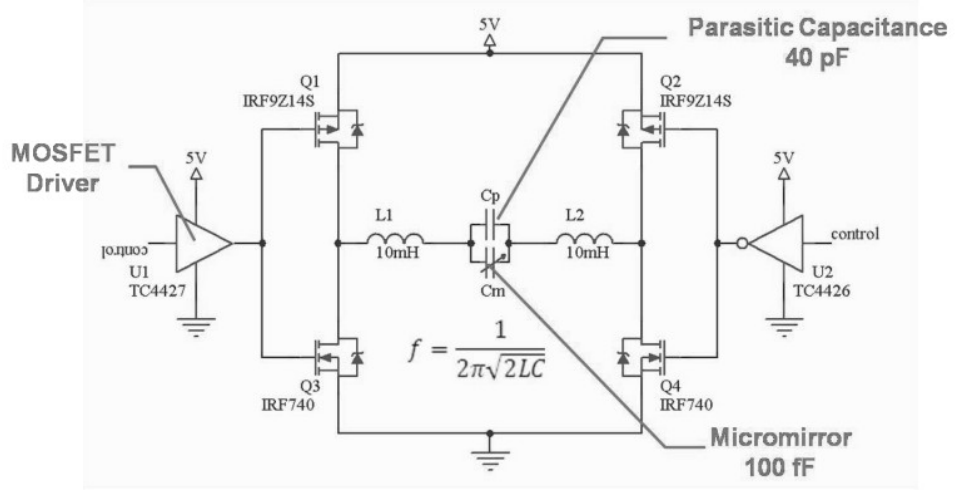

Figure 4.6 Balanced resonant drive circuit driven by an H-bridge and MOSFET drivers: U1 is a MOSFET driver with the non-inverting output; U2 is a MOSFET driver that has an inverting output.

Moreover, since the resonant drive circuit is excited by a square wave generated by the switching mechanism, an excitation signal can be represented as a sum of sinusoidal harmonics using the Fourier series as follows:

$$
x_{\text {square }}(t)=\frac{4}{\pi} \sum_{k}^{\infty} \frac{\sin \left(k \omega_{0} t\right)}{k}, k=1,3,5,7 \ldots
$$

The frequency components of the square wave that are higher than the initial resonant frequency $\omega_{0}$ are heavily attenuated by the resonant drive circuit itself, which also acts as a low pass filter, the frequency response of which is shown in Figure 4.3. Hence we can further simplify the excitation signal by ignoring higher frequency components of the square wave than the initial resonant frequency of the resonant drive circuit as follows: 


$$
x_{\text {excitation }}(t)=\frac{4}{\pi} \sin \left(\omega_{0} t\right)
$$

Hence, the final voltage gain obtained by the balanced resonant drive circuit with the H-bridge is equal to the voltage gain as thus:

$$
V_{\text {gain }}=\frac{4}{\pi} \frac{2 Q_{f} C_{0}(r+1)}{\sqrt{Q_{f}^{2}\left(C_{0}-C_{m}\right)^{2}+\left(C_{p}+C_{m}\right)^{2}}}
$$

Using the evaluated voltage gain, we derive the RMS value of the actuation voltage amplified by the balanced resonant drive circuit as follows:

$$
V_{r m s}=\frac{4 \sqrt{2} Q_{f} C_{0}(r+1)}{\pi \sqrt{Q^{2}\left(C_{0}-C_{m}\right)^{2}+\left(C_{p}+C_{m}\right)^{2}}} u
$$

Substituting the actuation voltage $\mathbf{V}$ in Eq. 3.4 with $\mathbf{V}_{\text {rms }}$ in Eq. 4.14 and normalizing $\mathbf{V}_{\text {rms }}$ in terms of the conventional pull-in voltage [9], we derive the non-dimensional governing equation of the parallel-plate actuator driven by the H-bridge as thus:

$$
\ddot{x}+2 \xi \omega_{n} \dot{x}+\omega_{m}{ }^{2} x=\frac{8}{27} \omega_{m}^{2} \frac{16 Q_{f}^{2}(r+1)^{2} \bar{u}^{2}}{\pi^{2}\left({Q_{f}}^{2} x^{2}+(r(1-x)+1)^{2}\right)}
$$

Due to the nature of the square-wave excitation signal, the H-bridge has $62 \%$ higher voltage gain than that of the op-amps in the BTL configuration. However, the on-resistance of the MOSFETs introduces more parasitic resistance in the signal path, resulting in the lower quality factor.

\subsubsection{Logic Gates in BTL Configuration}

The balanced resonant drive circuit implemented with the H-bridge is well suited for high voltage applications. However, its quality factor is reduced by the on-resistance of the MOSFETs, and its switching frequency is also restricted by the parasitic capacitance between the gate and source of the MOSFETs. Hence, we design the resonant drive circuit driven by two logic gates in the bridge-tied load configuration as presented in Figure 3.2.

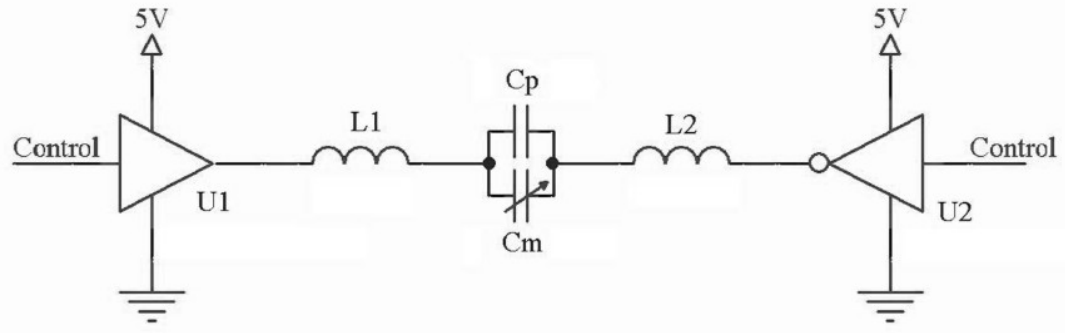

Figure 4.7 Balanced resonant drive circuit driven by two logic gates arranged in the BTL configuration: both logic gates have the push-pull output. 
This balanced resonant drive circuit with two logic gates is, in theory, identical to the balanced resonant drive circuit implemented with the H-bridge in the previous section, because the output of the logic gates is in the push-pull configuration, which is the same as an half H-bridge. However, the MOSFETs inside the logic gates are better matched during the fabrication process. Hence, their onresistance is lower than that of discrete MOSFETs, and the small parasitic capacitance allows higher switching frequency.

\subsection{Resonant Drive Circuit with Enhanced Inherent Negative Feedback}

As described in Chapter 3, the parasitic capacitance of an electrostatic actuator and the quality factor of the resonant drive circuit determine the region of the stable fixed points of the electrostatic actuator. In the presence of high parasitic capacitance, the resonant drive circuit fails to drive the parallel-plate actuator over its entire gap, although it is able to extend the operation range beyond the conventional pull-in point. To enhance the inherent negative feedback of the resonant drive circuit, we exploit the gain enhancement technique of an op-amp implemented with a positive feedback loop [51-53].

As the parallel-plate actuator displaces toward its bottom electrode, its capacitance increases. This increment in the capacitance not only reduces the quality factor of the resonant drive circuit, but increases the impedance of the resonant drive circuit, because the resonant drive circuit has the lowest impedance at its resonant frequency, as presented in Figure 4.8.

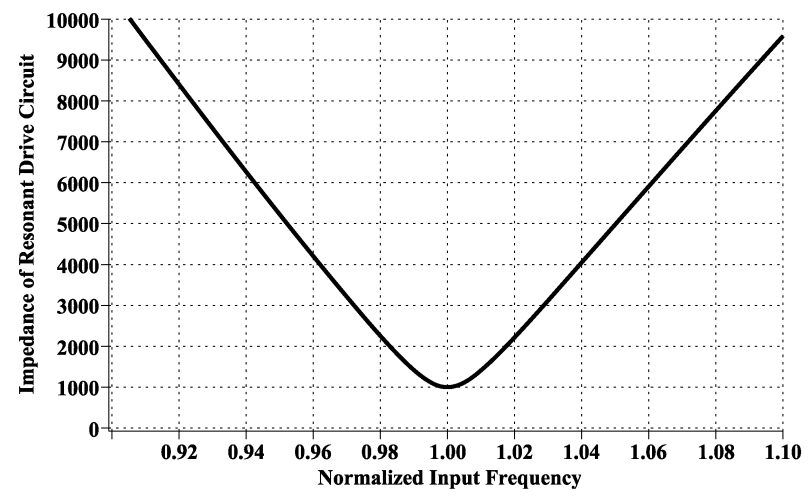

Figure 4.8 Impedance of the resonant drive circuit with respect to the input frequency: the input frequency is normalized with respect to the resonant frequency.

By putting the resonant drive circuit inside a positive feedback loop of an op-amp, we are able to reduce the voltage gain of an op-amp, stabilizing the parallel-plate actuator beyond its conventional pull-in point as the parallel-plate actuator displaces toward its bottom electrode. According to the opamp's golden rules [54], an op-amp is stable, as long as its negative feedback is stronger than its positive feedback. When its positive feedback is stronger than the negative feedback, an op-amp starts acting as an oscillator with a hysteresis. When the voltage of the positive input is bigger than that of the negative input, an op-amp saturates its output voltage to its positive power rail and when the voltage of the positive input is lower than that of the negative input, an op-amp drives its output voltage to the lowest voltage that is defined by its negative power rail. Since we want to operate an 
op-amp in its linear region, we have to make sure that its positive feedback is always weaker than the negative feedback of the op-amp.

Suppose that $R_{1}$ is equal to $R_{3}$ in Figure 4.9-(a). We can meet this stability condition by setting $R_{4}$ to be less than $R_{p}$, which is the parasitic resistance at the resonant frequency of the unbalanced resonant drive circuit presented in Figure 4.9-(b). Since the impedance of the unbalanced resonant drive circuit is always bigger than, or equal to the parasitic resistance $R_{p}$ over entire frequency ranges, this guarantees that this variable gain amplifier operates in a linear, stable region.

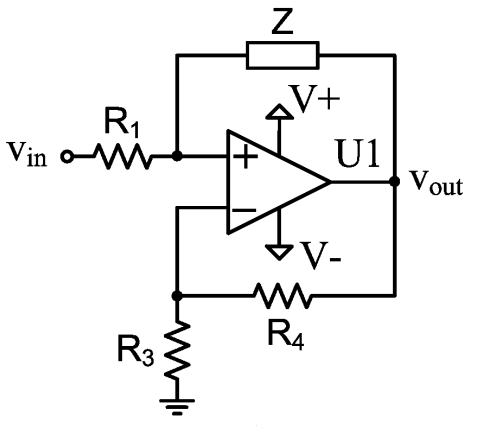

(a)

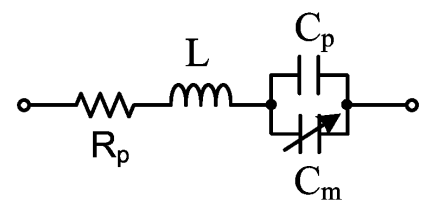

(b)

Figure 4.9 (a) Unbalanced resonant drive circuit placed within a positive feedback loop of an opamp that is configured as a non-inverting amplifier with the minimum gain of $\left(1+R_{4} / R_{3}\right)$ and (b) an unbalanced resonant drive circuit represented by $\mathrm{Z}$ in (a).

In order to determine the gain of this variable gain amplifier implemented with both feedback loops as presented in Figure 4.9, we use the op-amp's golden rules [54] with the assumption of the negative feedback being stronger than the positive feedback and evaluate the voltage of the positive and negative inputs of the op-amp as thus:

$$
\begin{aligned}
& V_{+}=\frac{R_{1}}{R_{1}+Z}\left(V_{\text {out }}-V_{\text {in }}\right)+V_{\text {in }} \\
& V_{-}=\frac{R_{3}}{R_{3}+R_{4}} V_{\text {out }}
\end{aligned}
$$

Since the op-amp drives its output to make both inputs have the same potential, we derive the voltage gain of the non-inverting amplifier connected with both the positive and negative feedback loops by solving Eq. 4.16 in terms of $\mathrm{V}_{\text {out }}$ and $\mathrm{V}_{\text {in }}$ as thus:

$$
V_{\text {out }}=\frac{Z}{R} \frac{\left(R+R_{4}\right)}{\left(Z-R_{4}\right)} V_{\text {in }}, \text { if } R=R_{1}=R_{3} \text { and }|Z|>R_{4}
$$

As presented in Eq. 4.17, the voltage gain of the non-inverting amplifier presented in Figure 4.9-(a) depends on the ratio of $Z$ to $R_{4}$, which determines the amount of the positive and negative feedbacks. If we assume that the ratio of $Z$ to $R_{4}$ is 1.1 , and $R_{1}, R_{3}$ and $R_{4}$ are of the same value, the voltage gain is 22. As shown in the left region of Figure 4.10, the voltage gain drops rapidly, when the impedance 
of $\mathrm{Z}$ increases with respect to $\mathrm{R}_{4}$. In other words, small increase in the impedance of $\mathrm{Z}$ results in huge loss in the voltage gain. We exploit this property of the non-inverting amplifier implemented with both feedback loops in order to stabilize the parallel-plate actuator coupled with the resonant drive circuit in the presence of high parasitic capacitance.

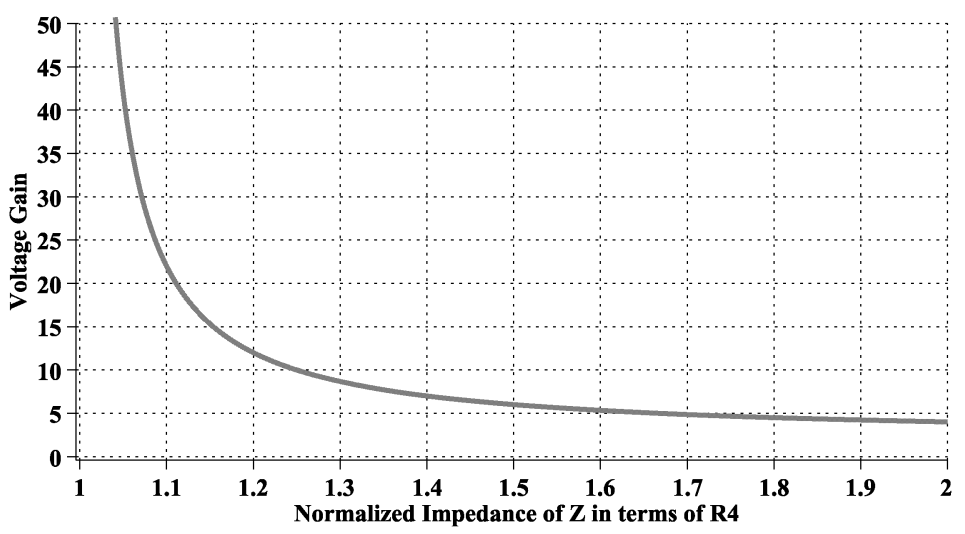

Figure 4.10 Voltage gain of the non-inverting amplifier in the previous figure in terms of the normalized impedance of $\mathrm{Z}$ with the condition of $\mathrm{R}_{1}=\mathrm{R}_{3}=\mathrm{R}_{4}$.

In order to verify that the mentioned stabilization scheme is feasible, we perform the numerical simulation on the non-inverting amplifiers implemented with both positive and negative feedback loops by using the analog electronic circuit simulators called LTspice IV and QUCS (Quite Universal Circuit Simulator): LTspice IV is a high performance circuit simulator based on SPICE (Simulation Program with Integrated Circuit Emphasis), being developed and distributed by Linear Technology; and QUCS is a very powerful circuit simulator as well, but does not make use of SPICE and has more distinctive features, such as the S-parameter measurement and the harmonic balance analysis.

The op-amps, U1 and U2, presented in Figure 4.11, are configured as a non-inverting amplifier with both negative and positive feedback loops in use. Because the negative feedback is stronger than the positive feedback in U1, U1 is stable and has a stable, fixed gain of 6 . The output voltage of U1, which is excited by the $1 \mathrm{~V}_{\mathrm{pp}}$ sinusoidal input signal at $10 \mathrm{~Hz}$, is presented in Figure 4.12. However, as presented in Figure 4.11-(b), the positive feedback of U2 is stronger than its negative feedback. Hence, the positive feedback drives the output of $\mathrm{U} 2$ into the saturation defined by either a positive or negative power supply. In other words, U2 acts as a bistable oscillator as the input voltage varies. Its SPICE simulation result is presented as a blue line in Figure 4.12. In practice, the op-amp, U2, is used as a comparator with a hysteresis, the amount of which is defined by a pair of the resistors, $R_{a}$ and $R_{b}$. 


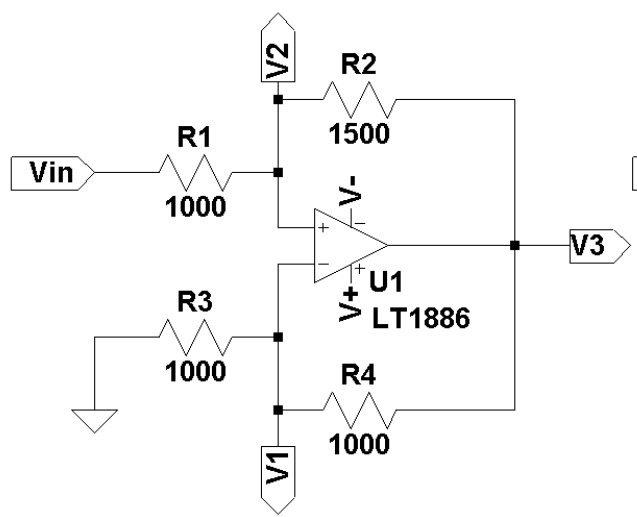

(a)

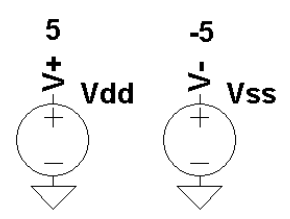

(c)

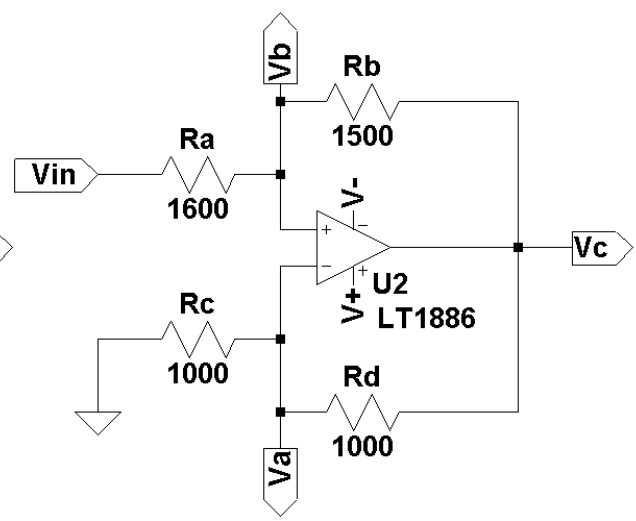

(b)

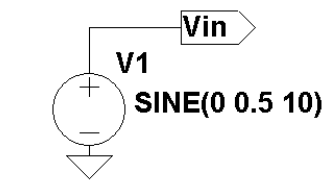

.tran 1 startup

(d)

Figure 4.11 Op-amps, U1 and U2, configured as a non-inverting amplifier with both negative feedback and positive feedback loops implemented: (a) the negative feedback is stronger than the positive feedback; (b) the positive feedback is stronger than the negative feedback; (c) U1 and U2 are powered by $5 \mathrm{~V}$ and $-5 \mathrm{~V}$; and $(\mathrm{d})$ an $1 \mathrm{~V}_{\mathrm{pp}}$ sinusoidal input signal without a DC bias at $10 \mathrm{~Hz}$.

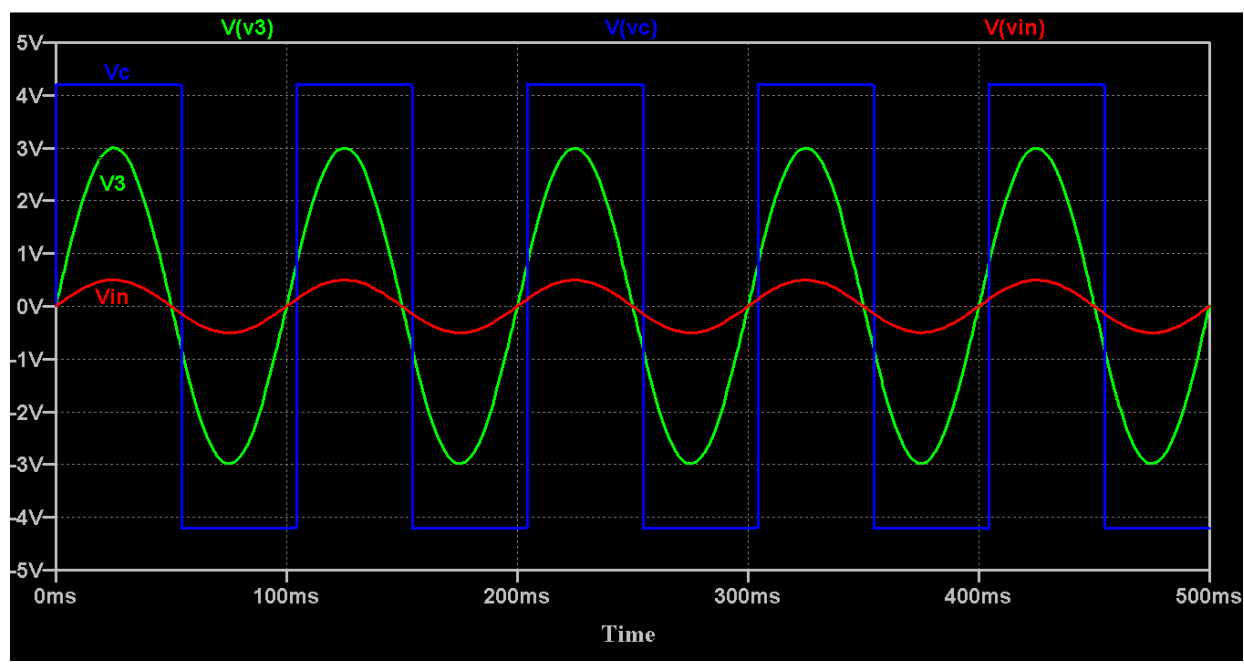

Figure 4.12 the SPICE simulation result of the circuits in the previous figure: the red solid line, Vin, represents $1 \mathrm{~V}_{\mathrm{pp}}$ sinusoidal input signal at $10 \mathrm{~Hz}$; the green solid line, $\mathrm{V} 3$, denotes the output of $\mathrm{U} 1$ in the previous figure; and the blue solid line, Vc, represents the output of U2 in the same figure. 
In order to determine the fixed points of the parallel-plate actuator and their stability, we derive the governing equation of the parallel-plate actuator coupled with the unbalanced resonant drive circuit, which is placed within the positive feedback loop of the non-inverting amplifier.

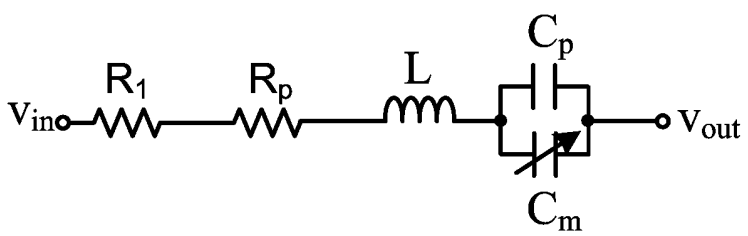

Figure 4.13 Simplified schematic of the unbalanced resonant drive circuit placed within a positive feedback loop of an op-amp, the output voltage of which is equal to $\mathrm{V}_{\text {out. }}$.

The governing equation of the unbalanced resonant drive circuit in Figure 4.13 is derived as thus:

$$
L C \frac{d^{2} V_{a}}{d t^{2}}+\left(R_{1}+R_{p}\right) C \frac{d V_{a}}{d t}+V_{a}=V_{\text {in }}-V_{\text {out }}
$$

where $\mathbf{C}$ represents the sum of $\mathbf{C}_{\mathbf{p}}$ and $\mathbf{C}_{\mathbf{m}} ; \mathbf{V}_{\text {in }}$ denotes the input voltage; $\mathbf{V}_{\text {out }}$ represents the output voltage of the non-inverting amplifier; and $\mathbf{V}_{\mathbf{a}}$ denotes the actuation voltage across the capacitors.

Substituting $\mathbf{V}_{\text {out }}$ in Eq. 4.18 with $\mathbf{V}_{\text {out }}$ in Eq. 4.17 and setting $\mathbf{R}_{\mathbf{1}}$ to be equal to $\mathbf{R}_{\mathbf{3}}$, we simplify Eq. 4.18 as thus:

$$
L C \frac{d^{2} V_{a}}{d t^{2}}+\left(R+R_{p}\right) C \frac{d V_{a}}{d t}+V_{a}=\frac{R_{4}(R+Z)}{R\left(R_{4}-Z\right)} V_{i n}
$$

Using the frequency response of Eq. 4.19, we derive the voltage gain of the resonant drive circuit driven by the non-inverting amplifier in Figure 4.9-(a) as thus:

$$
H(j \omega)=\frac{R_{4}(R+Z)}{R\left(R_{4}-Z\right)} \frac{1}{1-L C \omega^{2}+j \omega\left(R+R_{p}\right) C}
$$

Since the frequency of the input signal is fixed at the initial resonant frequency of the resonant drive circuit, we determine the impedance of the positive feedback loop at the initial resonant frequency as thus:

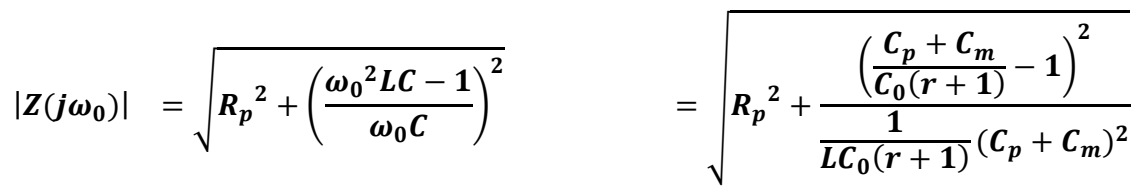

$$
\begin{aligned}
& =\sqrt{R_{p}{ }^{2}+\frac{\left(\frac{C_{p}+C_{m}-C_{0}(r+1)}{C_{0}(r+1)}\right)^{2}}{\frac{\left(C_{p}+C_{m}\right)^{2}}{L C_{0}(r+1)}}}=\sqrt{R_{p}{ }^{2}+\frac{L\left(C_{m}-C_{0}\right)^{2}}{C_{0}(r+1)\left(C_{p}+C_{m}\right)^{2}}}
\end{aligned}
$$


Because the capacitance of the parallel-plate actuator is represented by its initial capacitance and normalized displacement as in Eq. 4.22, we simplify Eq. 4.21 into Eq. 4.23:

$$
\begin{gathered}
C_{m}=\frac{\varepsilon A}{g_{0}(1-x)}=\frac{C_{0}}{(1-x)}, Q_{f}=\sqrt{\frac{L}{R_{p}{ }^{2} C_{0}(r+1)}} \\
\left|Z\left(j \omega_{0}\right)\right|=\sqrt{\frac{R_{p}{ }^{2}+\frac{L\left(\frac{1}{1-x}-1\right)^{2}}{C_{0}(r+1)\left(\frac{r(1-x)+1}{1-x}\right)^{2}}}{R^{2}}}=\sqrt{R_{p}{ }^{2}+\frac{L x^{2}}{C_{0}(r+1)(r(1-x)+1)^{2}}} \\
=\sqrt{R_{p}{ }^{2}+\frac{R_{p}{ }^{2} L x^{2}}{R_{p}{ }^{2} C_{0}(r+1)(r(1-x)+1)^{2}}}=\sqrt{R_{p}{ }^{2}+\frac{L}{R_{p}{ }^{2} C_{0}(r+1)} \frac{R_{p}{ }^{2} x^{2}}{(r(1-x)+1)^{2}}} \\
=\sqrt{R_{p}{ }^{2}\left(1+\frac{Q_{f}{ }^{2} x^{2}}{(r(1-x)+1)^{2}}\right)}
\end{gathered}
$$

Using the impedance of $\mathrm{Z}$ at the initial resonant frequency in Eq. 4.23, we determine the variable voltage gain of the non-inverting amplifier in terms of the normalized displacement of the parallelplate actuator and the minimum gain of the amplifier as thus:

$$
\begin{aligned}
\frac{R_{4}\left(R+\left|Z\left(j \omega_{0}\right)\right|\right)}{R\left(R_{4}-\left|Z\left(j \omega_{0}\right)\right|\right)}= & \frac{R_{4}}{R} \frac{\left(R+\sqrt{R_{p}{ }^{2}\left(1+\frac{Q_{f}^{2} x^{2}}{(r(1-x)+1)^{2}}\right)}\right)}{\left(R_{4}-\sqrt{R_{p}^{2}\left(1+\frac{Q_{f}^{2} x^{2}}{(r(1-x)+1)^{2}}\right)}\right)} \\
& =\frac{R_{4}}{R} \frac{\left(R+R_{p} \frac{\sqrt{Q_{f}^{2} x^{2}+(r(1-x)+1)^{2}}}{(r(1-x)+1)}\right)}{\left(R_{4}-R_{p} \frac{\sqrt{Q_{f}^{2} x^{2}+(r(1-x)+1)^{2}}}{(r(1-x)+1)}\right)} \\
& =\frac{R_{4}}{R} \frac{\left(R(r(1-x)+1)+R_{p} \sqrt{Q_{f}^{2} x^{2}+(r(1-x)+1)^{2}}\right)}{\left(R_{4}(r(1-x)+1)-R_{p} \sqrt{Q_{f}^{2} x^{2}+(r(1-x)+1)^{2}}\right)}
\end{aligned}
$$

Using the same method of the frequency response, we determine the voltage gain of the unbalanced resonant drive circuit itself at its initial resonant frequency in terms of the normalized displacement, the initial quality factor, the parasitic capacitance, and the minimum gain set by $\mathbf{R}$ and $\mathbf{R}_{\mathbf{4}}$ in Figure 4.9-(a) as thus: 


$$
\begin{aligned}
\left|\frac{1}{1-L C \omega^{2}+j \omega\left(R+R_{p}\right) C}\right|_{\omega=\omega_{0}} & =\left|\frac{1}{1-\frac{C_{p}+C_{m}}{C_{0}(r+1)}+j \frac{\left(R+R_{p}\right)\left(C_{p}+C_{m}\right)}{\sqrt{L C_{0}(r+1)}} \mid}\right| \\
& =\frac{1}{\sqrt{\frac{x^{2}}{(r+1)^{2}(1-x)^{2}}+\frac{\left(R+R_{p}\right)^{2} C_{0}\left(r+\frac{1}{1-x}\right)^{2}}{L(r+1)}}} \\
& =\frac{1}{\sqrt{\frac{x^{2}}{(r+1)^{2}(1-x)^{2}}+\frac{\left(R+R_{p}\right)^{2} C_{0}(r(1-x)+1)^{2}}{L(r+1)(1-x)^{2}}}} \\
& =\frac{1}{\sqrt{\frac{x^{2}}{(r+1)^{2}(1-x)^{2}}+\frac{\left(R+R_{p}\right)^{2} R_{p}^{2} C_{0}(r+1)}{R_{p}^{2}} \frac{(r(1-x)+1}{(r+1)^{2}(1-x)^{2}}}} \\
& =\frac{\sqrt{\frac{Q_{f}^{2} x^{2}+\left(1+\frac{R}{R_{p}}\right)^{2}(r(1-x)+1)^{2}}{x^{2}}}}{\sqrt{\frac{x^{2}}{(r+1)^{2}(1-x)^{2}}+\left(1+\frac{R}{R_{p}}\right)^{2} \frac{(r(1-x)+1)^{2}}{Q_{f}^{2}(r+1)^{2}(1-x)^{2}}}}
\end{aligned}
$$

Combining Eq. 4.24 and Eq. 4.25, we derive the total voltage gain of the resonant drive circuit excited by the non-inverting amplifier implemented with both feedback loops as thus:

$$
\begin{gathered}
\left|H\left(j w_{0}\right)\right|=\frac{R_{4}}{R} \frac{\left(R(r(1-x)+1)+R_{p} \sqrt{Q_{f}^{2} x^{2}+(r(1-x)+1)^{2}}\right)}{\left(R_{4}(r(1-x)+1)-R_{p} \sqrt{Q_{f}^{2} x^{2}+(r(1-x)+1)^{2}}\right)} \\
\times \frac{Q_{f}(r+1)(1-x)}{\sqrt{{Q_{f}}^{2} x^{2}+\left(1+\frac{R}{R_{p}}\right)^{2}(r(1-x)+1)^{2}}}
\end{gathered}
$$

Finally, using the voltage gain in Eq. 4.26, we derive the normalized governing equation of the parallel-plate actuator coupled with the resonant drive circuit, which is placed in the positive feedback loop of the non-inverting amplifier, as follows: 


$$
\begin{gathered}
\ddot{x}+2 \xi \omega_{n} \dot{x}+\omega_{m}{ }^{2} x \\
=\frac{2}{27} \omega_{m}^{2}\left(\frac{R_{4}}{R}\right)^{2} \frac{\left(R(r(1-x)+1)+R_{p} \sqrt{Q_{f}^{2} x^{2}+(r(1-x)+1)^{2}}\right)^{2}}{\left(R_{4}(r(1-x)+1)-R_{p} \sqrt{Q_{f}^{2} x^{2}+(r(1-x)+1)^{2}}\right)^{2}} \\
\times \frac{Q_{f}{ }^{2}(r+1)^{2}}{Q_{f}{ }^{2} x^{2}+\left(1+\frac{R}{R_{p}}\right)^{2}(r(1-x)+1)^{2}} \bar{u}^{2}
\end{gathered}
$$

Using Eq. 4.27, we determine the fixed points of the parallel-plate actuator and their stability in the presence of high parasitic capacitances, when the parallel-plate actuator coupled with the resonant drive circuit is actuated by the non-inverting amplifier presented in Figure 4.9. The circuit parameters used to set the voltage gain of the non-inverting amplifier in Figure 4.9 are presented in the following table:

Table 4.1 Circuit parameters used to set the minimum voltage gain of the non-inverting amplifier

\begin{tabular}{ccc}
\hline Parameter & Symbol & Value \\
\hline \hline & $\mathrm{R}_{1}$ & $100,1000[\Omega]$ \\
& $\mathrm{R}_{3}$ & $100,1000[\Omega]$ \\
& $\mathrm{R}_{4}$ & $2000[\Omega]$ \\
Parasitic resistance of the resonant drive circuit & $\mathrm{R}_{\mathrm{p}}$ & $2100[\Omega]$ \\
Initial quality factor of the resonant drive circuit & $\mathrm{Q}_{\mathrm{f}}$ & 50 \\
Ratio of $\mathrm{C}_{\mathrm{p}}$ to $\mathrm{C}_{0}$ & $\mathrm{r}$ & $100,200,400$ \\
Minimum gain of the non-inverting amplifier & & 21,3 \\
\hline \hline
\end{tabular}

When the parallel-plate actuator coupled with the resonant drive circuit is excited by the noninverting amplifier implemented with both feedback loops, its fixed points are presented in Figure 4.14 and Figure 4.15, where the solid lines represent the stable fixed points with different parasitic capacitances and the dotted lines denote the location of the saddle nodes. Since each solid line does not intersect with the corresponding dotted line, the parallel-plate actuator does not undergo the saddle-node bifurcation and has more robustness to the pull-in phenomenon. The fixed points of the parallel-plate actuator in Figure 4.14 are obtained when the minimum gain of the non-inverting amplifier is set to be 21. The fixed points in Figure 4.15 are obtained from the non-inverting amplifier, the minimum gain of which is set to be 3. Although there is big difference in the minimum gain of the non-inverting amplifiers, their operation ranges are almost identical except for the input voltage, which is adjusted by the minimum voltage gain. Moreover, by setting R4 appropriately, we are able to stabilize the parallel-plate actuator over its entire gap in the presence of high parasitic capacitance. 


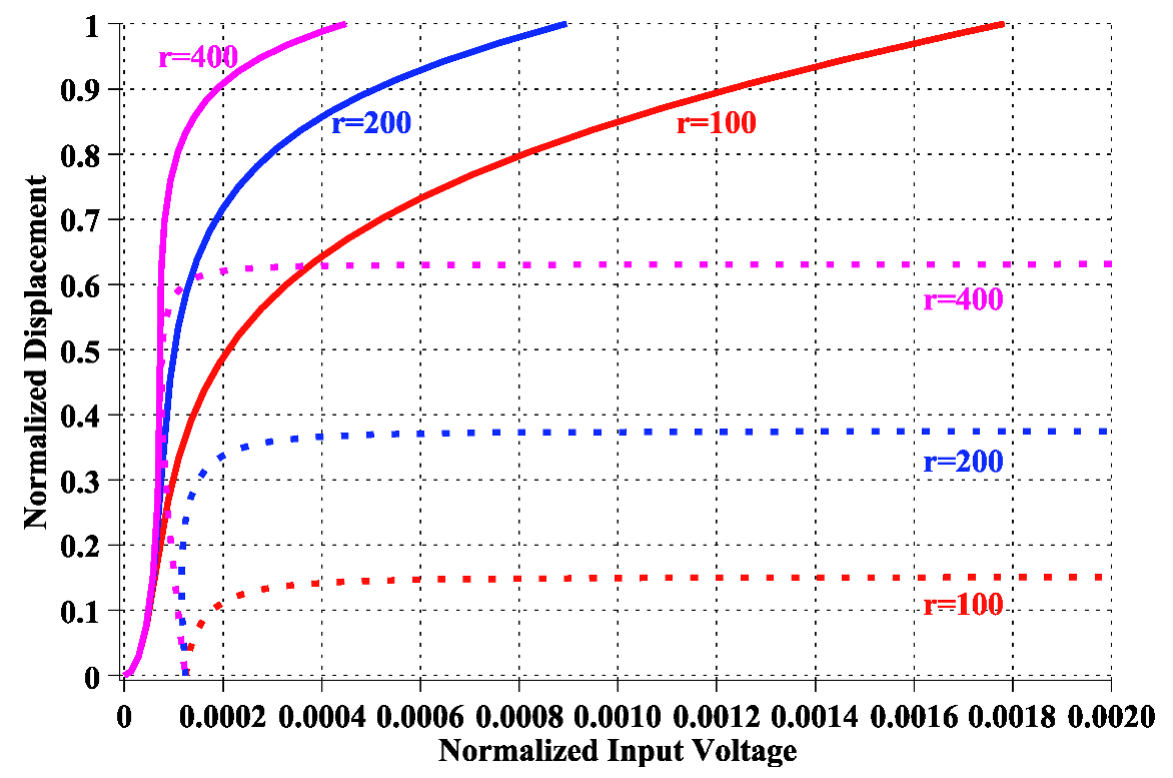

Figure 4.14 Fixed points of the parallel-plate actuator driven by the resonant drive circuit arranged in the positive feedback loop of the non-inverting amplifier with the following parameters: $\mathrm{R}_{4}=2000 \Omega, \mathrm{R}_{\mathrm{p}}=2100 \Omega, \mathrm{Q}_{\mathrm{f}}=50$, and $\mathrm{R}_{1}=\mathrm{R}_{3}=100 \Omega$.

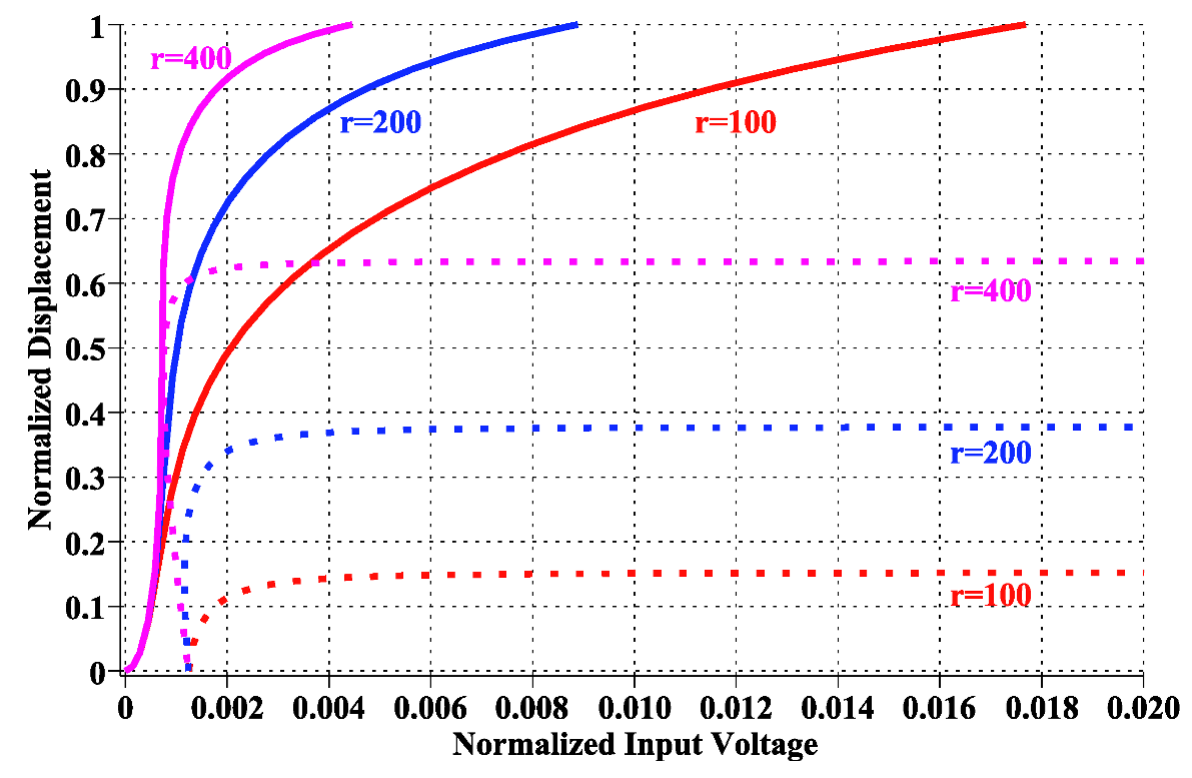

Figure 4.15 Fixed points of the parallel-plate actuator driven by the resonant drive circuit arranged in the positive feedback loop of the non-inverting amplifier with the following parameters: $\mathrm{R}_{4}=2000 \Omega, \mathrm{R}_{\mathrm{p}}=2100 \Omega, \mathrm{Q}_{\mathrm{f}}=50$, and $\mathrm{R}_{1}=\mathrm{R}_{3}=1000 \Omega$. 
The saddle nodes of the parallel-plate actuator are also evaluated in terms of its parasitic capacitance to determine its operation range, when it is driven by the non-inverting amplifier in Figure 4.9. In Figure 4.16, the red solid line represents the saddle nodes of the parallel-plate actuator driven by the unbalanced resonant drive circuit with the initial quality factor $\mathbf{Q}_{\mathbf{f}}$ of 50 . The blue dashed line and the black dotted line denote the saddle nodes of the parallel-plate actuator coupled with the unbalanced resonant drive circuit driven by the non-inverting amplifier with $\mathbf{Q}_{\mathrm{f}}=50, \mathbf{R}_{4}=2000 \Omega$, and $\mathbf{R}_{1}=\mathbf{R}_{3}=100 \Omega$ : the blue dashed line is obtained with the parameters of $\mathbf{R}_{\mathbf{p}}=2100 \Omega$; and the black dotted line is acquired with $\mathbf{R}_{\mathbf{p}}=2010 \Omega$.

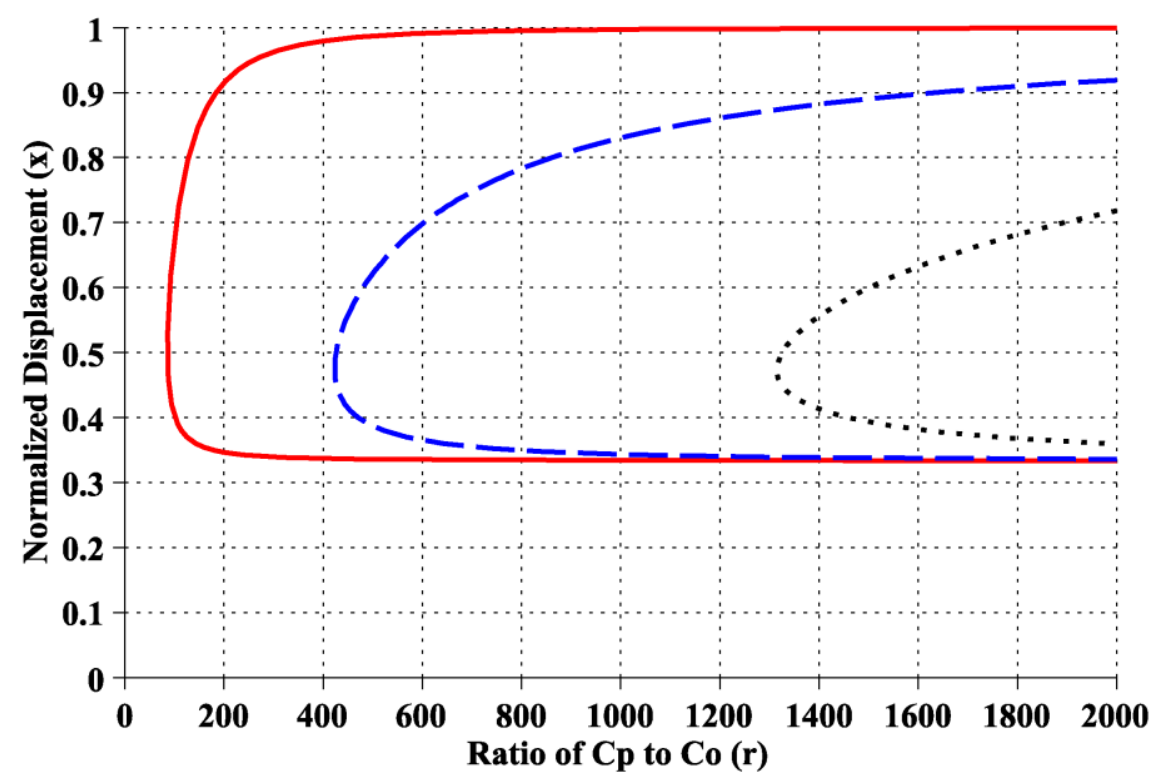

Figure 4.16 Saddle nodes of the parallel-plate actuator coupled with the unbalanced resonant drive circuit driven by the non-inverting amplifier implemented with both negative and positive feedback loops.

As presented in Figure 4.16, the non-inverting amplifier implemented with both negative and positive feedback loops in Figure 4.9 is able to extend the operation range of the parallel-plate actuator with very high parasitic capacitance as long as the resonant frequency remains within the bandwidth of the amplifier in use.

\subsection{Summary}

We present different ways of implementing the resonant drive circuits with discrete electronics components in this chapter: the unbalanced resonant drive circuit driven by the unity-gain amplifier and the balanced resonant drive circuits driven by two op-amps in the BTL configuration, the Hbridge with complementary MOSFET drivers, and logic gates in the BTL configuration. The balanced resonant drive circuit achieves higher voltage gain, but has lower quality factor than those of the unbalanced resonant drive circuit. Furthermore, the balanced resonant drive circuits implemented with the H-bridge and the logic gates in the BTL configuration do not require an AC voltage source 
and are well suited for the frequency modulation control. In Section 4.3, we present that the resonant drive circuit placed in the positive feedback loop of the non-inverting amplifier has more robustness to the saddle-node bifurcation even in the presence of very high parasitic capacitance by exploiting the gain change caused by the impedance variation due to the displacement of the parallel-plate actuator. We also derive the analytical model of each resonant drive circuit to determine the fixed points of the parallel-plate actuator in terms of the quality factor of the resonant drive circuit and the parasitic capacitance of the parallel-plate actuator. Using these analytical models, we are able to determine the fixed points of the parallel-plate actuator, when it is driven by various resonant drive circuit described in this chapter. 


\section{Chapter 5}

\section{Microbeams Actuated by Resonant Drive Circuit}

Among many electrostatic actuators, a microbeam is also one of the most popular electrostatic actuators: a cantilever beam implemented as a mass sensor; a fixed-fixed microbeam as a RF switch; and a microplate supported by a pair of microbeams as a RF demodulator. In this chapter, we demonstrate through the finite element analysis and the experiment that the resonant drive circuits described in the previous chapters are capable of driving these microbeams at lower supply voltage while extending the operation range of the microbeams in the presence of high parasitic capacitance.

This chapter is organized as follows. In Section 5.1, we create FEM of a cantilever beam and derive its fixed points through numerical simulation while it is driven by voltage control. We use the same FEM of the cantilever beam to analyze its fixed points while it is actuated by the resonant drive circuit, which is implemented as an algebraic constraint on its boundary condition. In Section 5.2, we also develop FEM of a fixed-fixed microbeam and analyze it to derive its fixed points while it is driven by both conventional voltage control and the resonant drive circuit. In Section 5.3, we present FEM of a microplate supported by two microbeams and its numerical results, when it is driven by conventional voltage control. The same process is repeated on the microplate, when it is actuated by the resonant drive circuit. Finally, the experiment results on the microplate are presented and compared with its numerical results for validation.

\subsection{Cantilever Beam Coupled with Resonant Drive Circuit}

In this section, we derive the governing equation of the cantilever beam presented in Figure 5.1 and create its finite element model to analyze and compare its static responses, when it is actuated by the conventional voltage control and the resonant drive circuit in order to validate the feasibility of the resonant drive circuit.

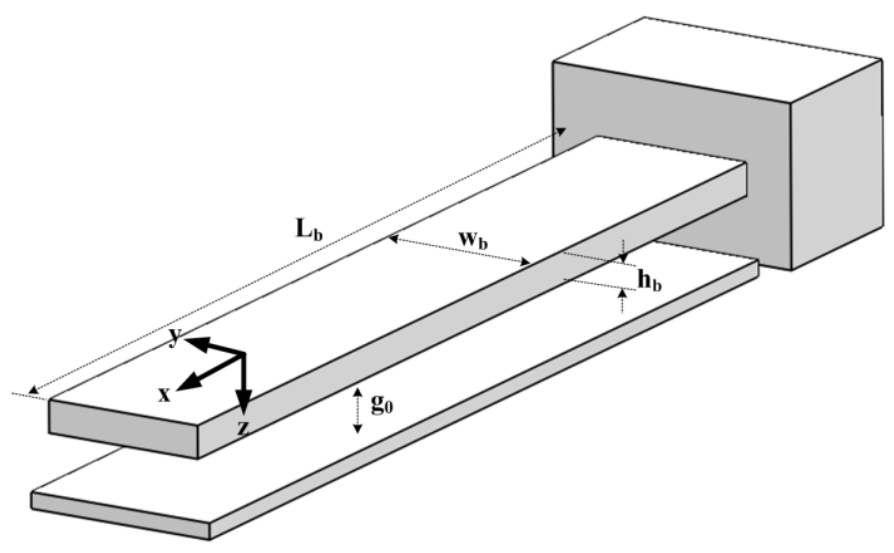

Figure 5.1 Isometric view of the cantilever beam, which is clamped on the right end, with its bottom electrode. 


\subsubsection{Analytical Model of Cantilever Beam}

The governing equation of the cantilever beam driven by the conventional voltage control is derived from the Euler-Bernoulli beam equation with its boundary conditions and initial conditions as thus [55]:

$$
\begin{gathered}
E I \frac{\partial^{4} w}{\partial x^{4}}+\rho A_{b} \frac{\partial^{2} w}{\partial t^{2}}+b \frac{\partial w}{\partial t}=\left(N+\frac{E A_{b}}{2 L_{b}} \int_{0}^{L_{b}}\left(\frac{\partial w}{\partial x}\right)^{2} d x\right) \frac{\partial^{2} w}{\partial x^{2}}+q \\
w(0, t)=0, \quad \frac{\partial^{2} w\left(L_{b}, t\right)}{\partial x^{2}}=0 \\
w^{\prime}(0, t)=0, \quad \frac{\partial^{3} w\left(L_{b}, t\right)}{\partial x^{3}}=0 \\
w(x, 0)=0, \quad \dot{w}(x, 0)=0
\end{gathered}
$$

where $\mathbf{E}$ represents the elastic modulus; I denotes the area moment of inertia of the cantilever beam; $\mathbf{w}$ is the function of $\mathbf{x}$ and $\mathbf{t}$, representing the displacement of the cantilever beam in the $\mathrm{z}$ axis along the length of the cantilever beam; $\boldsymbol{\rho}$ stands for the density of the cantilever beam; $\mathbf{A}_{\mathbf{b}}$ denotes the cross-sectional area of the cantilever beam; $\mathbf{b}$ denotes the viscous damping per unit length; $\mathbf{N}$ represents an axial load applied on the cantilever beam; $\mathbf{L}_{\mathbf{b}}$ represents the length of the cantilever beam; and $\mathbf{q}$ denotes the distributed load on the cantilever beam, which is equal to the electrostatic force per unit length applied on the cantilever beam as presented in Eq. 5.3:

$$
q=\frac{1}{2} \varepsilon w_{b} \frac{V^{2}}{\left(g_{0}-w\right)^{2}}
$$

where $\boldsymbol{\varepsilon}$ represents the permittivity of air; $\mathbf{w}_{\mathbf{b}}$ denotes the width of the cantilever beam; $\mathbf{V}$ represents the potential difference between the cantilever beam and its bottom electrode; and $\mathbf{g}_{\mathbf{0}}$ denotes the initial gap at rest; and $\mathbf{w}$ is the displacement of the cantilever beam in the $\mathrm{z}$ axis along its length.

Combining Eq. 5.1 and Eq. 5.3, we have the governing equation of the cantilever beam driven by voltage control as thus:

$$
E I \frac{\partial^{4} w}{\partial x^{4}}+\rho A_{b} \frac{\partial^{2} w}{\partial t^{2}}+b \frac{\partial w}{\partial t}=\left(N+\frac{E A_{b}}{2 L_{b}} \int_{0}^{L_{b}}\left(\frac{\partial w}{\partial x}\right)^{2} d x\right) \frac{\partial^{2} w}{\partial x^{2}}+\frac{1}{2} \varepsilon w_{b} \frac{V^{2}}{\left(g_{0}-w\right)^{2}}
$$

We also derive the governing equation of the cantilever beam coupled with the resonant drive circuit by using the same multiple scale method described in Chapter 3 as thus:

$$
\begin{aligned}
E I \frac{\partial^{4} w}{\partial x^{4}}+\rho A_{b} \frac{\partial^{2} w}{\partial t^{2}}+b \frac{\partial w}{\partial t} & =\left(N+\frac{E A_{b}}{2 L_{b}} \int_{0}^{L_{b}}\left(\frac{\partial w}{\partial x}\right)^{2} d x\right) \frac{\partial^{2} w}{\partial x^{2}} \\
& +\frac{1}{4} \frac{\varepsilon w_{b}}{\left(g_{0}-w\right)^{2}} \frac{\left(Q_{f} C_{0}(r+1)\right)^{2} u^{2}}{Q_{f}^{2}\left(C_{0}-C_{m}\right)^{2}+\left(C_{p}+C_{m}\right)^{2}}
\end{aligned}
$$


where $\mathbf{Q}_{\mathbf{f}}$ represents the initial quality factor of the resonant drive circuit; $\mathbf{C}_{\mathbf{0}}$ denotes the initial capacitance of the cantilever beam at rest; $\mathbf{r}$ represents the ratio of the parasitic capacitance $\mathbf{C}_{\mathbf{p}}$ to $\mathbf{C}_{\mathbf{0}}$; $\mathbf{C}_{\mathbf{m}}$ denotes the capacitance of the cantilever beam represented by the following equation:

$$
C_{m}=\int_{0}^{L_{b}} \frac{\varepsilon w_{b}}{g_{0}-w} d x
$$

\subsubsection{Finite Element Model of Cantilever Beam}

We create the finite element model of the cantilever beam in Figure 5.1 with the physical parameters presented in Table 5.1 in order to verify the feasibility of the resonant drive circuit, which is implemented as a nonlinear boundary condition on the bottom side of the cantilever beam.

Table 5.1 Parameters of the cantilever beam used in FEA

\begin{tabular}{ccc}
\hline \hline Parameter & Symbol & Value \\
\hline Material & & PolySi \\
Material density & $\rho$ & $2320\left[\mathrm{~kg} / \mathrm{m}^{3}\right]$ \\
\hline Elastic modulus of PolySi & $\mathrm{E}$ & $160[\mathrm{GPa}]$ \\
Poisson's ration & $v$ & 0.23 \\
\hline Length of the cantilever beam & $\mathrm{L}_{\mathrm{b}}$ & $300[\mu \mathrm{m}]$ \\
\hline $\begin{array}{c}\text { Width of the cantilever beam } \\
\text { Thickness of the fixed-fixed } \\
\text { microbeam }\end{array}$ & $\mathrm{w}_{\mathrm{b}}$ & $50[\mu \mathrm{m}]$ \\
Initial gap & $\mathrm{h}_{\mathrm{b}}$ & $2[\mu \mathrm{m}]$ \\
Pull-in voltage & $\mathrm{g}_{0}$ & $2[\mu \mathrm{m}]$ \\
$\begin{array}{c}\text { Initial quality factor } \\
\text { of the resonant drive circuit }\end{array}$ & $\mathrm{V}_{\mathrm{pi}}$ & $6.32[\mathrm{~V}]$ \\
Ratio of $\mathrm{C}_{\mathrm{p}}$ to $\mathrm{C}_{0}$ & $\mathrm{Q}_{\mathrm{f}}$ & 100 \\
\hline \hline
\end{tabular}

The displacement of the cantilever beam is presented in Figure 5.2, when the cantilever beam is driven by conventional voltage control and the resonant drive circuit, the quality factor of which is set to be 100 . The parasitic capacitance of the cantilever beam is set to be 100 as well. Although there is not much improvement of the operation range of the cantilever beam, the resonant drive circuit displaces the cantilever beam at much lower voltage than that of voltage control. The main reason why the cantilever beam coupled with the resonant drive circuit does not have an extended operation range is that most parts of the cantilever beam do not displace much as its tip displaces toward its bottom electrode. 
The cantilever beam in Figure 5.2 undergoes the saddle-node bifurcation at $0.781 \mu \mathrm{m}$ at $6.32 \mathrm{~V}$, when it is driven by conventional voltage control. It displaces $0.977 \mu \mathrm{m}$ at the input voltage of $0.187 \mathrm{~V}$ while being driven by the resonant drive circuit with the following parameters: $\mathbf{Q}_{\mathrm{f}}=100$ and $\mathbf{r}=100$.

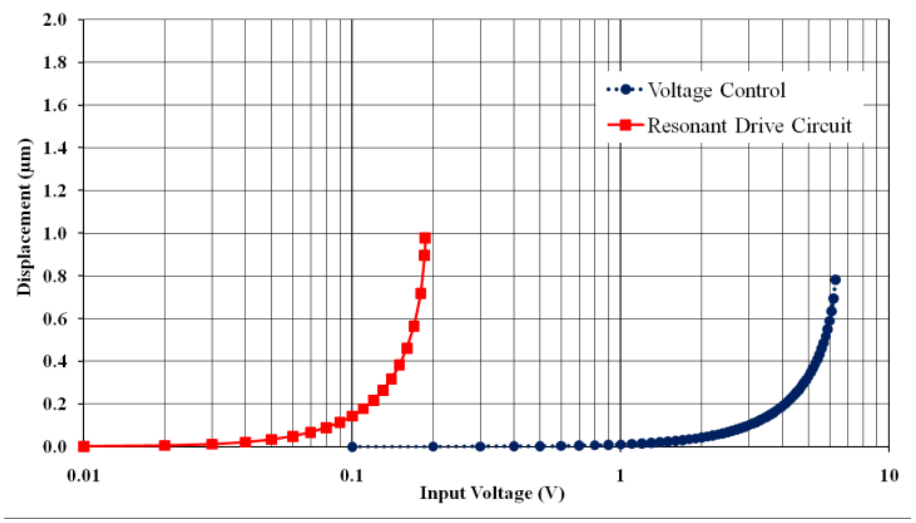

Figure 5.2 Displacement of the cantilever beam actuated by the resonant drive circuit (the red solid squares) and conventional voltage control (the blue solid circles) with the following parameters: the initial quality factor $\mathbf{Q}_{\mathrm{f}}=100$, the parasitic capacitance $\mathbf{C}_{\mathbf{p}}=100 \mathbf{C}_{\mathbf{0}}$.

The cantilever beam post-processed with its displacement in the $\mathrm{z}$ axis is presented in Figure 5.3, when it is drive by the unbalanced resonant drive circuit with the following parameters: $\mathbf{Q}_{\mathrm{f}}=100$ and $\mathbf{C}_{\mathbf{p}}=50 \mathbf{C}_{\mathbf{0}}$. The cantilever beam undergoes the saddle-node bifurcation at $1.473 \mu \mathrm{m}$ at the input voltage of $0.233 \mathrm{~V}$. The displacement of the cantilever beam at various input voltage is presented in Figure 5.4 .

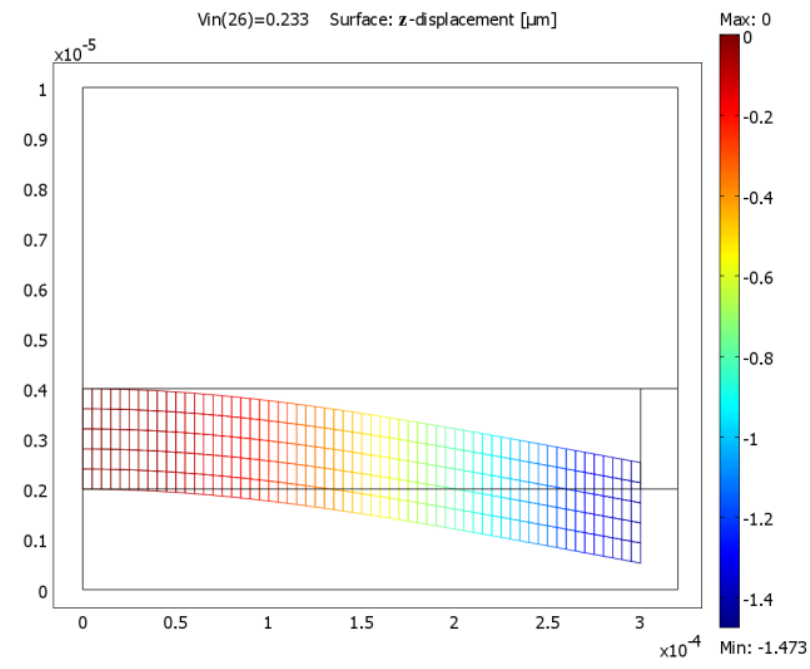

Figure 5.3 Cantilever beam post-processed with its displacement in the $\mathrm{z}$ axis while being actuated by the resonant drive circuit with the following parameters: the initial quality factor $\mathbf{Q}_{\mathrm{f}}=100$, the parasitic capacitance $\mathbf{C}_{\mathbf{p}}=50 \mathbf{C}_{\mathbf{0}}$, and the peak input voltage of $0.233 \mathrm{~V}$. 


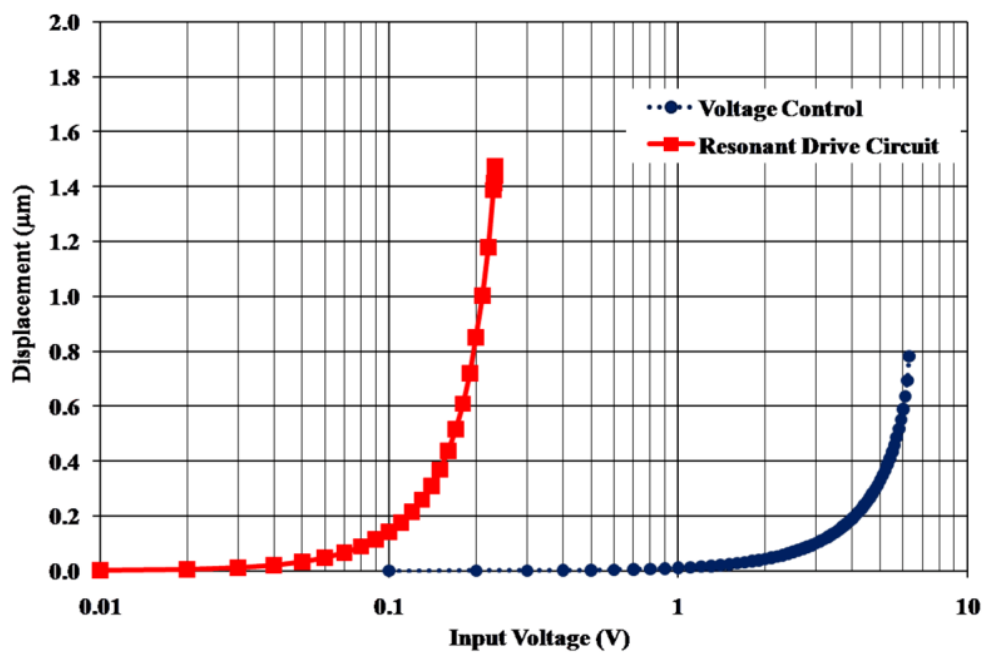

Figure 5.4 Displacement of the cantilever beam actuated by the resonant drive circuit (the red solid squares) and conventional voltage control (the blue solid circles) with the following parameters: the initial quality factor $\mathbf{Q}_{\mathrm{f}}=100$, the parasitic capacitance $\mathbf{C}_{\mathbf{p}}=50 \mathbf{C}_{\mathbf{0}}$.

\subsection{Fixed-Fixed Beam Driven by Resonant Drive Circuit}

In this section, we present a finite element model of the fixed-fixed microbeam depicted in Figure 5.5, and analyze its displacement when it is driven by both conventional voltage control and the resonant drive circuit to validate the feasibility of the resonant drive circuit.

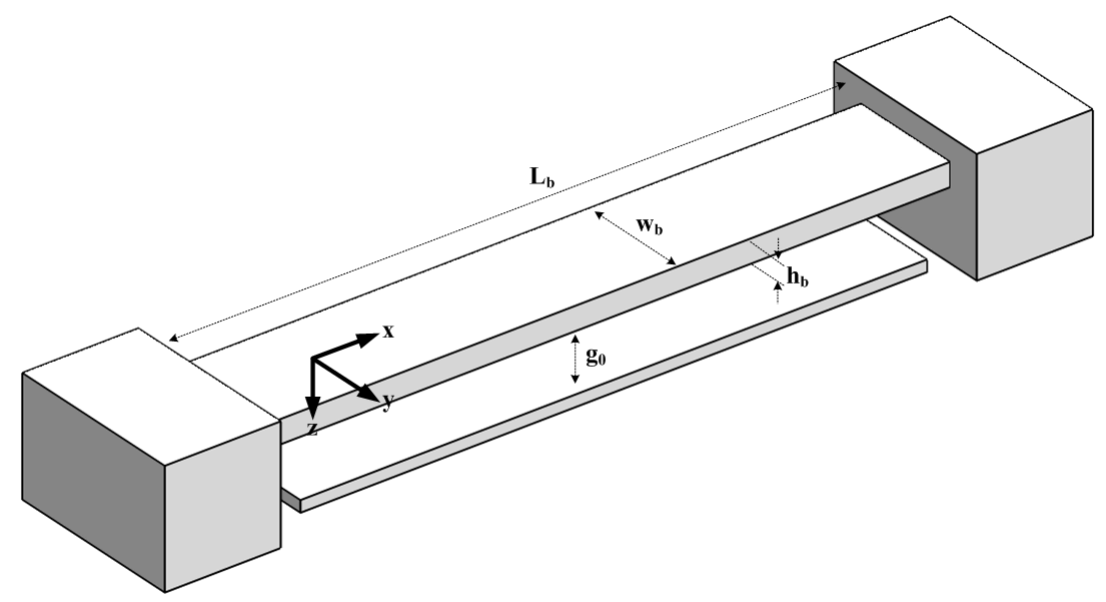

Figure 5.5 Isometric view of the fixed-fixed microbeam that has both ends clamped at the anchors.

We derive the governing equation and boundary conditions of the fixed-fixed microbeam using the Euler-Bernoulli beam equation [55] as shown in Eq. 5.7 and Eq. 5.8. 


$$
\begin{aligned}
& E I \frac{\partial^{4} w}{\partial x^{4}}+\rho A_{b} \frac{\partial^{2} w}{\partial t^{2}}+b \frac{\partial w}{\partial t}=\left(N+\frac{E A_{b}}{2 L_{b}} \int_{0}^{L_{b}}\left(\frac{\partial w}{\partial x}\right)^{2} d x\right) \frac{\partial^{2} w}{\partial x^{2}} \\
&+\frac{1}{4} \frac{\varepsilon w_{b}}{\left(g_{0}-w\right)^{2}} \frac{\left(Q_{f} C_{0}(r+1)\right)^{2} u^{2}}{Q_{f}^{2}\left(C_{0}-C_{m}\right)^{2}+\left(C_{p}+C_{m}\right)^{2}} \\
& w(0, t)=0, \quad w\left(L_{b}, t\right)=0 \\
& w^{\prime}(0, t)=0, \quad w^{\prime}\left(L_{b}, t\right)=0
\end{aligned}
$$

The following parameters in Table 5.2 are used to define the properties of the fixed-fixed microbeam and the resonant drive circuit.

Table 5.2 Parameters of the fixed-fixed microbeam used in FEA

\begin{tabular}{ccc}
\hline Parameter & Symbol & Value \\
\hline \hline Material & & PolySi \\
Material density & $\rho$ & $2320\left[\mathrm{~kg} / \mathrm{m}^{3}\right]$ \\
\hline Elastic modulus of PolySi & $\mathrm{E}$ & $160[\mathrm{GPa}]$ \\
Poisson's ration & $v$ & 0.23 \\
\hline Length of the fixed-fixed microbeam & $\mathrm{L}_{\mathrm{b}}$ & $300[\mu \mathrm{m}]$ \\
\hline Width of the fixed-fixed microbeam & $\mathrm{w}_{\mathrm{b}}$ & $50[\mu \mathrm{m}]$ \\
\hline $\begin{array}{c}\text { Thickness of the fixed-fixed } \\
\text { microbeam }\end{array}$ & $\mathrm{h}_{\mathrm{b}}$ & $1[\mu \mathrm{m}]$ \\
\hline $\begin{array}{c}\text { Axial tensile load } \\
\text { Initial gap }\end{array}$ & $\mathrm{N}$ & $10[\mathrm{MPa}]$ \\
Pull-in voltage & $\mathrm{g}_{0}$ & $2.5[\mu \mathrm{m}]$ \\
Initial quality factor & $\mathrm{V}_{\mathrm{pi}}$ & $32[\mathrm{~V}]$ \\
of the resonant drive circuit & $\mathrm{Q}_{\mathrm{f}}$ & 100 \\
Ratio of $\mathrm{C}_{\mathrm{p}}$ to $\mathrm{C}_{0}$ & $\mathrm{r}$ & 100 \\
\hline \hline
\end{tabular}

The fixed-fixed microbeam post-processed with its displacement is presented in Figure 5.6, when it is driven by the unbalanced resonant drive circuit. The displacement of the fixed-fixed microbeam at various input voltage is presented in Figure 3.2 for comparison, when it is drive by both conventional voltage control and the resonant drive circuit.

The simulation results verify that the resonant drive circuit is not only able to extend the operation range of the fixed-fixed microbeam beyond its conventional pull-in point, but also requires much less input voltage to displace the microbeam before the saddle-node bifurcation occurs. For instance, when the fixed-fixed microbeam driven by conventional voltage control undergoes the saddle-node bifurcation at $32 \mathrm{~V}$, its displacement is $1.24 \mu \mathrm{m}$. However, the resonant drive circuit extends its operation range up to $2.145 \mu \mathrm{m}$ at the input voltage of $1.23 \mathrm{~V}$. 


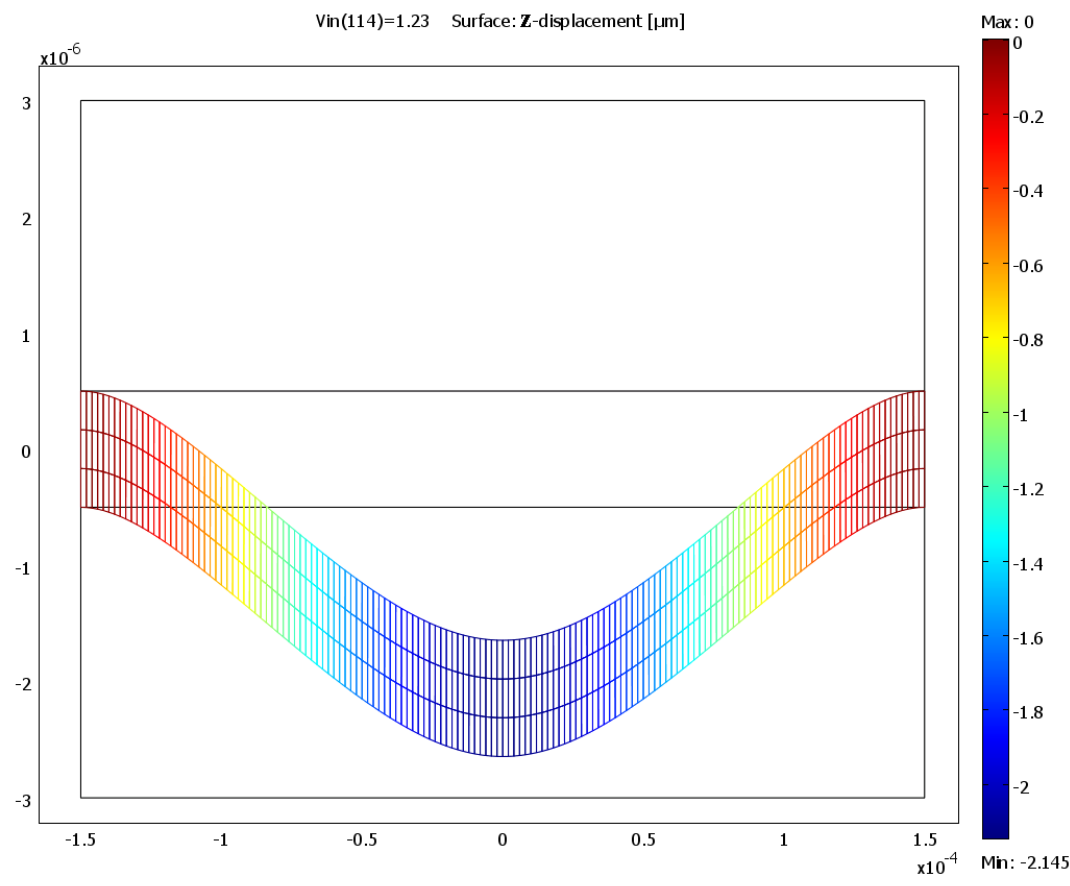

Figure 5.6 Fixed-fixed microbeam post-processed with the displacement in the $\mathrm{z}$ axis while being actuated by the resonant drive circuit with the following parameters: the initial quality factor $\mathrm{Q}_{\mathrm{f}}=100$, the parasitic capacitance $\mathrm{C}_{\mathrm{p}}=100 \mathrm{C}_{0}$, and the peak input voltage $1.23 \mathrm{~V}$.

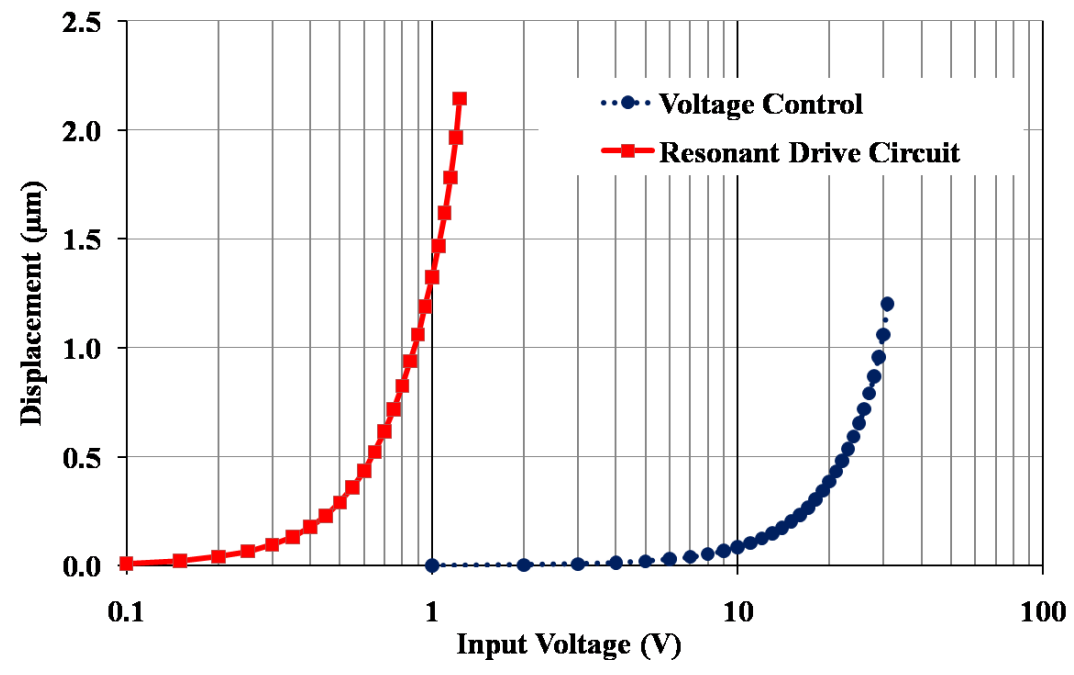

Figure 5.7 Stable fixed points of the fixed-fixed microbeam at various input voltages when driven by conventional voltage control and the resonant drive circuit with the initial quality factor $\mathrm{Q}_{\mathrm{f}}=100$ and the parasitic capacitance $\mathrm{C}_{\mathrm{p}}=100 \mathrm{C}_{0}$. 


\subsection{Microplate Supported by Two Microbeams and Actuated by Resonant Drive Circuit}

In this section, we present the numerical results and the experiment results of the microplate guided by two microbeams shown in Figure 5.8, when the microplate is drive by conventional voltage control and the resonant drive circuit.

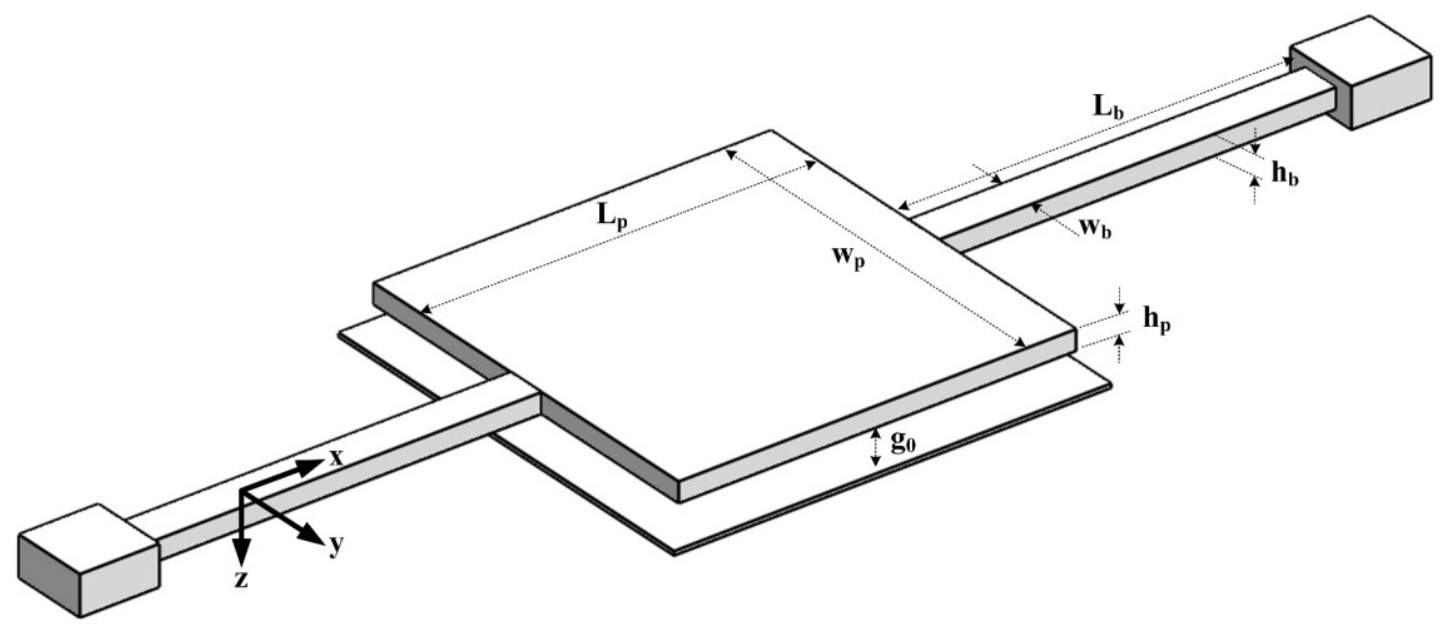

Figure 5.8 Isometric view of the microplate supported by a pair of microbeams attached to anchors.

The actual microplate supported by two microbeams is fabricated through PolyMUMPs, and its pictures acquired by an optical microscope and a profilometer are presented in Figure 5.9.

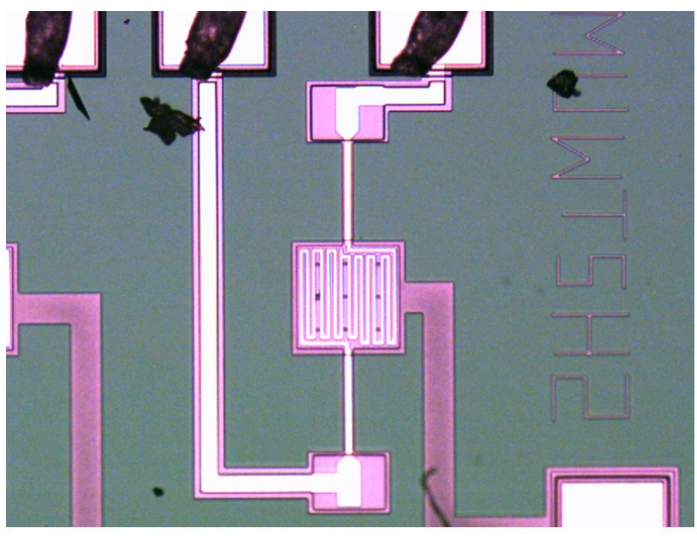

(a)

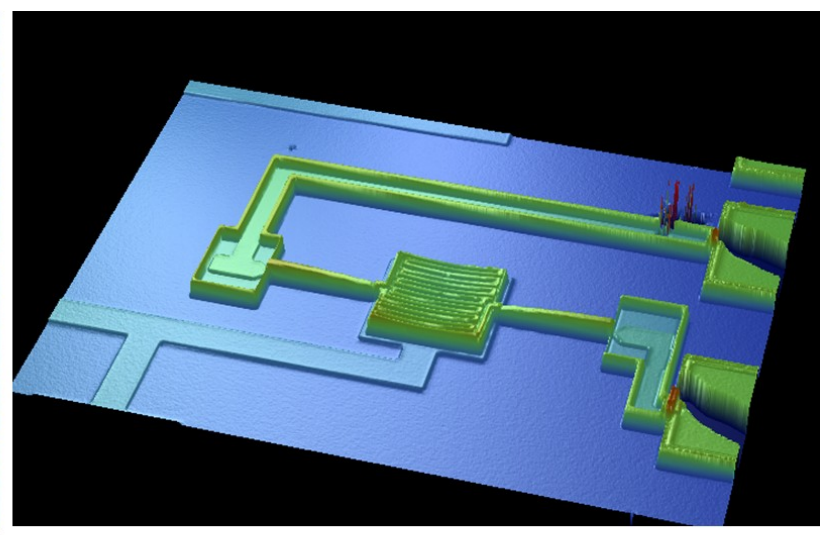

(b)

Figure 5.9 (a) Top view of the microplate supported by two microbeams obtained through an optical microscope and (b) a false-color image of the same microplate acquired by a profilometer. 


\subsubsection{Finite Element Model of Microplate Guided by Two Microbeams}

We create the finite element model of the microplate supported by two identical microbeams in COMSOL, based on the physical properties of the actual device, which are shown in Table 5.3.

Table 5.3 Physical properties of the microplate supported by two microbeams

\begin{tabular}{ccc}
\hline \hline Parameter & Symbol & Value \\
\hline \hline Material & & PolySi \\
Material density & $\rho$ & $2320\left[\mathrm{~kg} / \mathrm{m}^{3}\right]$ \\
Elastic modulus of PolySi & $\mathrm{E}$ & $160[\mathrm{GPa}]$ \\
Width of the microplate & $\mathrm{w}_{\mathrm{p}}$ & $100[\mu \mathrm{m}]$ \\
Length of the microplate & $\mathrm{L}_{\mathrm{p}}$ & $100[\mu \mathrm{m}]$ \\
Thickness of the microplate & $\mathrm{h}_{\mathrm{p}}$ & $1.5[\mu \mathrm{m}]$ \\
Width of the microbeam & $\mathrm{w}_{\mathrm{b}}$ & $10[\mu \mathrm{m}]$ \\
Length of the microbeam & $\mathrm{L}_{\mathrm{b}}$ & $100[\mu \mathrm{m}]$ \\
Thickness of the microbeam & $\mathrm{h}_{\mathrm{b}}$ & $1.5[\mu \mathrm{m}]$ \\
Initial gap & $\mathrm{g}_{0}$ & $1.14[\mu \mathrm{m}]$ \\
Initial capacitance & $\mathrm{C}_{0}$ & $77.7[\mathrm{fF}]$ \\
\hline \hline
\end{tabular}

The displacement profiles of the microplate are presented in Figure 5.10, when it is driven by conventional voltage control. The microplate post-processed with its displacement at the input voltage of $6.84 \mathrm{~V}$ is shown in Figure 5.11.

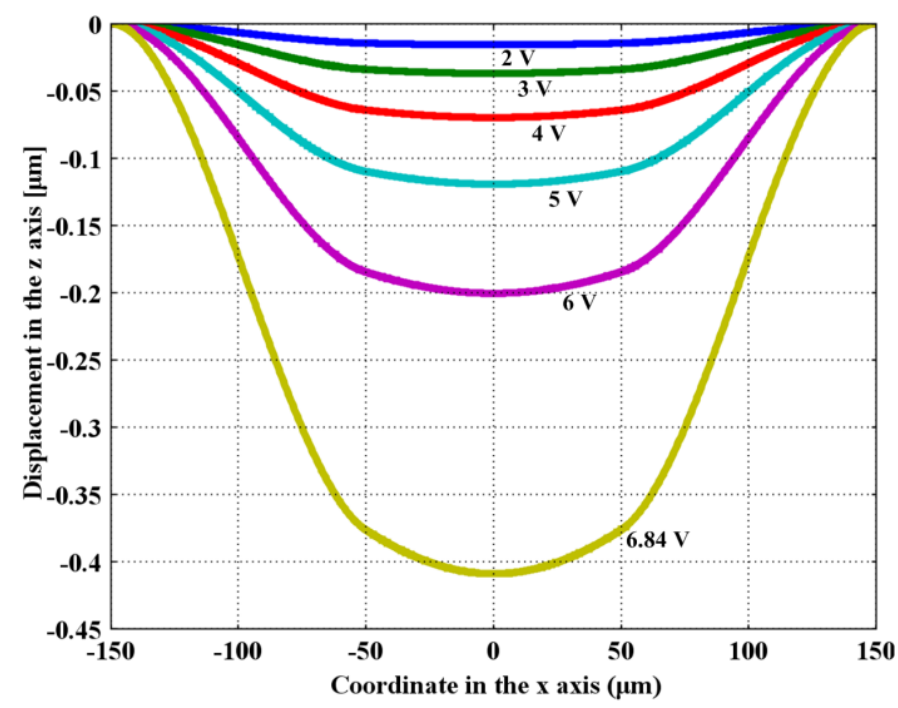

Figure 5.10 Displacement profiles of the microplate supported by two microbeams at various actuation voltages: the pull-in voltage of the microplate is $6.84 \mathrm{~V}$. 


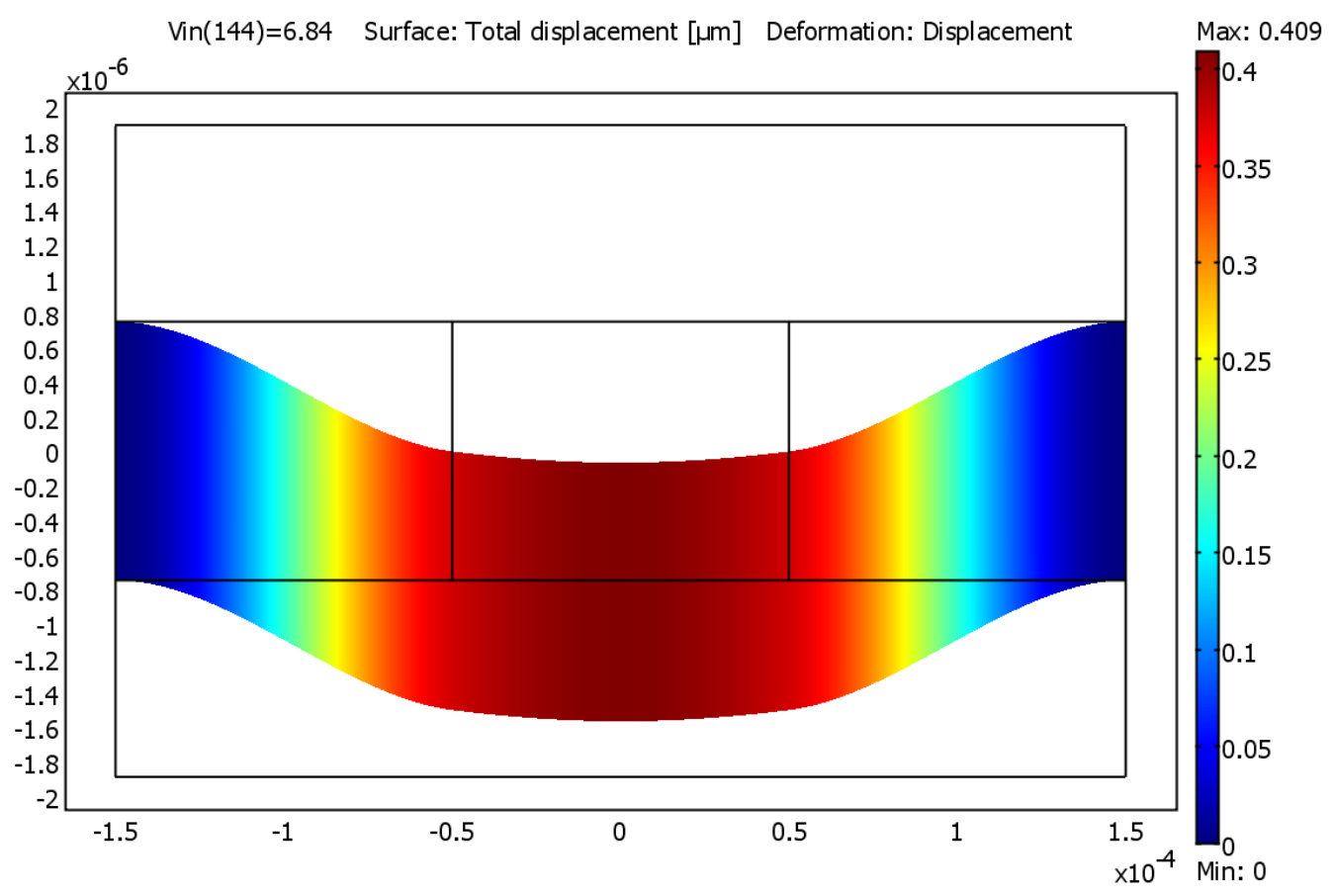

Figure 5.11 Microplate post-processed with its displacement at the actuation voltage of $6.84 \mathrm{~V}$ before the pull-in occurs: the displacement of the center of the microplate is $0.409 \mu \mathrm{m}$.

\subsubsection{Experiment Results on Microplate Guided by Two Microbeams}

In order to verify the design parameters of the microplate with a pair of the microbeams, we inspect the microplate under an optical microscope and measure the displacement of the microplate by using a vibrometer while a function generator drives the microplate. We use an oscilloscope to capture the input voltage, the displacement signal from the DD-200 displacement decoder of the OFV-5000 vibrometer from Polytec, and the velocity signal from the VD-02 velocity decoder of the same vibrometer. The displacement and velocity of the microplate measured by the vibrometer are presented in Figure 5.12 and Figure 5.13.

In Figure 5.12, we actuate the microplate with the $8 \mathrm{~V}_{\mathrm{pp}}$ sinusoidal signal with the DC bias of $4 \mathrm{~V}$ at 1 $\mathrm{Hz}$. Its measured displacement and velocity clearly show when the microplate undergoes the saddlenode bifurcation and pulls out after its contact on the bottom electrode. The measured pull-in voltage of the microplate is $6.57 \mathrm{~V}$, and its displacement is $0.39 \mu \mathrm{m}$ at the pull-in. The voltage at which the microplate comes out of the contact is $1.867 \mathrm{~V}$. In Figure 5.13, we drive the same microplate with the $8 \mathrm{~V}_{\mathrm{pp}}$ triangular signal with the DC bias of $4 \mathrm{~V}$ at $1 \mathrm{~Hz}$ in order to determine its pull-in voltage and pull-in point. The measured displacement clearly represents its nonlinear response to the triangular input signal. The measured velocity clearly indicates how fast the microplate undergoes the saddlenode bifurcation as well. The measured pull-in voltage of the microplate is $6.57 \mathrm{~V}$ in average, and its displacement is $0.39 \mu \mathrm{m}$ at the pull-in. 


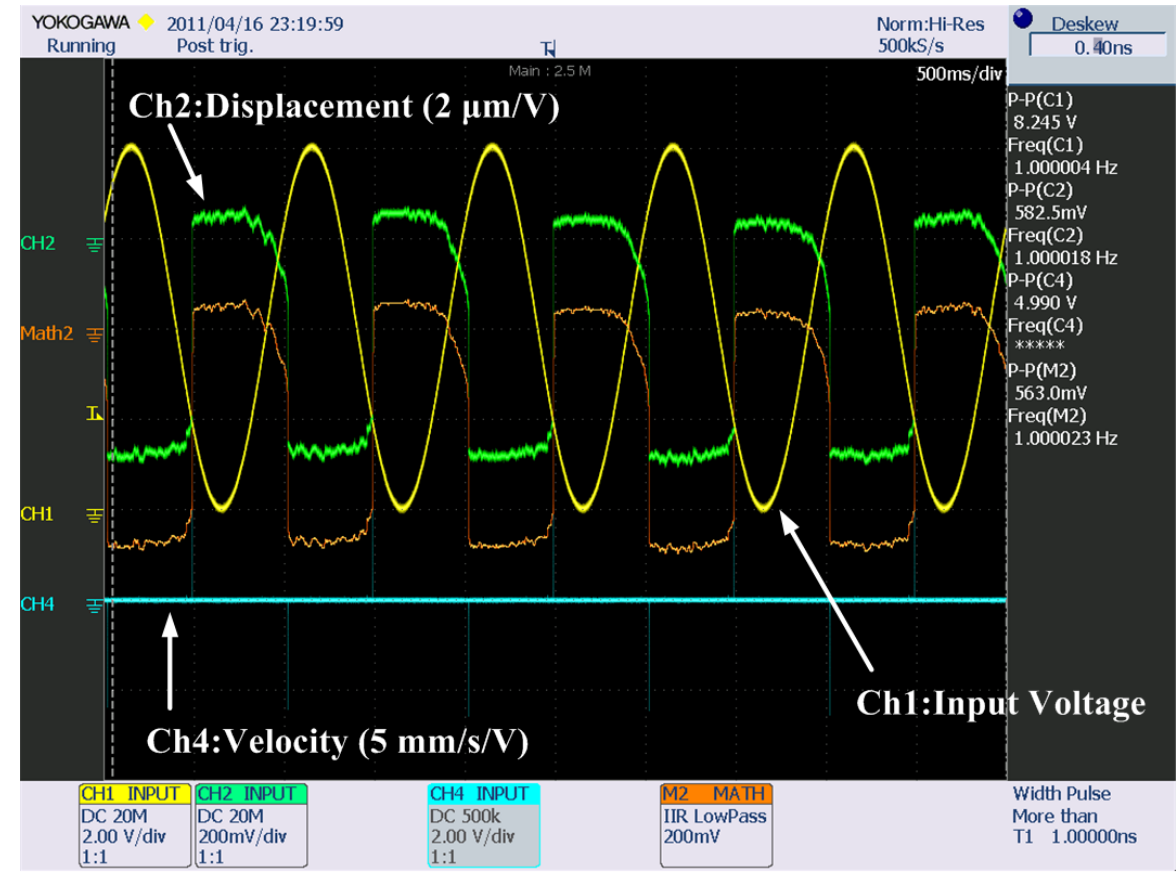

Figure 5.12 Measured displacement and velocity of the microplate excited by the $8 \mathrm{~V}_{\mathrm{pp}}$ sinusoidal signal with the DC bias of $4 \mathrm{~V}$ at $1 \mathrm{~Hz}: \mathrm{V}_{\mathrm{pi}}=6.57 \mathrm{~V}$ at $0.39 \mu \mathrm{m}$ and $\mathrm{V}_{\mathrm{po}}=1.867 \mathrm{~V}$.

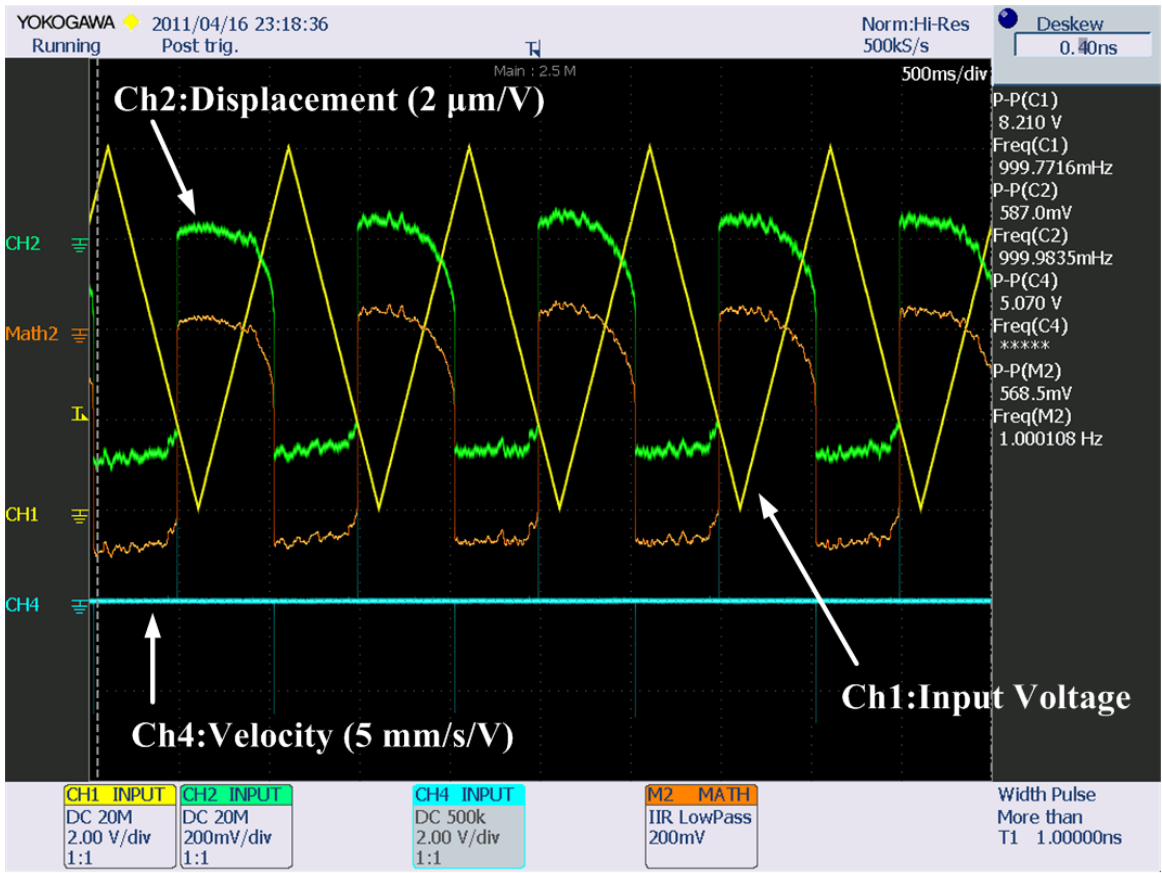

Figure 5.13 Measured displacement and velocity of the microplate excited by the $8 \mathrm{~V}_{\mathrm{pp}}$ triangular signal with the DC bias of $4 \mathrm{~V}$ at $1 \mathrm{~Hz}$ : $\mathrm{V}_{\mathrm{pi}}=6.57 \mathrm{~V}$ at $0.39 \mu \mathrm{m}$ and $\mathrm{V}_{\mathrm{po}}=1.867 \mathrm{~V}$. 
In Figure 5.14-(a), the displacement of the microplate and the input voltage to the microplate are presented against time. These experiment results are compared to the numerical results obtained from its finite element model in Figure 5.14-(b), where the red solid squares represent the displacement of the microplate from its FEM, and the blue solid circles denote the displacement of the microplate obtained from its experiment. Since the dynamics of the contact behavior is not part of FEM of the microplate, we are not able to analyze the behavior of the microplate after it makes contact on its bottom electrode. However, we are currently working on the complete analytical and numerical models of the microplate, including its contact behavior. As presented in Figure 5.14-(b), the numerical results show that the microplate undergoes the saddle-node bifurcation at $6.84 \mathrm{~V}$ and its displacement is $0.41 \mu \mathrm{m}$ at the pull-in. We believe that this difference might be explained by the fridge field of the microplate, because of the size of the microplate and the small gap between the microplate and its bottom electrode.

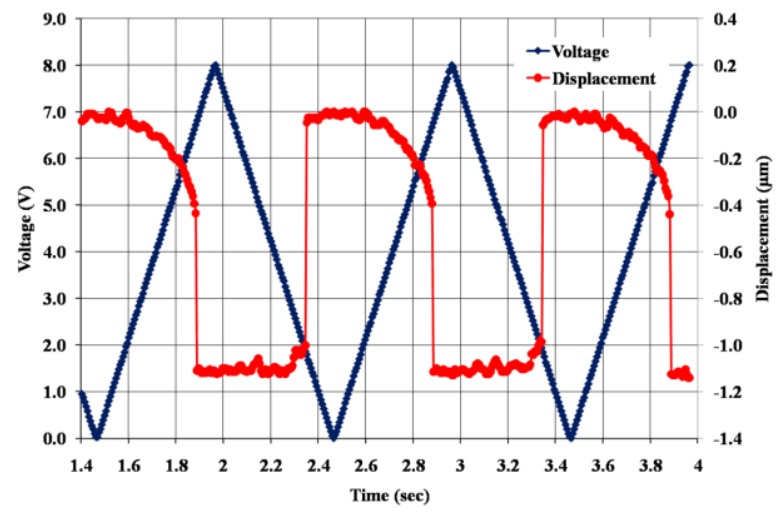

(a)

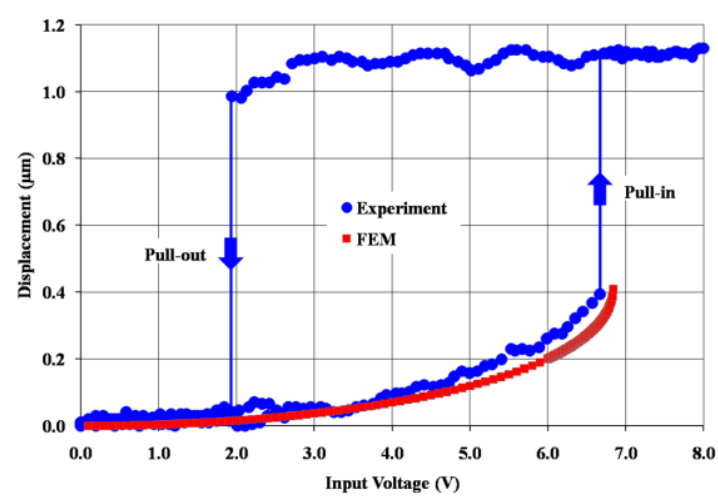

(b)

Figure 5.14 Displacement of the microplate from the experiment and its FEA in COMSOL: the experiment result shows $\mathrm{V}_{\mathrm{pi}}=6.57 \mathrm{~V}$ at $0.39 \mu \mathrm{m}$; and FEA determines $\mathrm{V}_{\mathrm{pi}}=6.84 \mathrm{~V}$ at $0.41 \mu \mathrm{m}$.

We also measure the displacement of the microplate actuated by the resonant drive circuit, which is excited by an amplitude modulator that is, in turn, driven by a control signal, also known as a baseband signal in RF applications. Due to the limitation of the amplitude modulator we use, we are not able to push the microplate into the rest state. As a result, there is a DC bias in the displacement of the microplate, which cannot be measured by the DD-200 displacement decoder. We present the screenshot of the oscilloscope that measures the modulated input voltage to the resonant drive circuit, the actuation voltage amplified by the resonant drive circuit, and the displacement signal coming out of the DD-200 displacement decoder in Figure 5.15.

As presented in Figure 5.15, the input signal is modulated by the amplitude modulator and its voltage is $321 \mathrm{mV}_{\mathrm{pp}}$. The actuation voltage amplified by the resonant drive circuit is $12 \mathrm{~V}_{\mathrm{pp}}$, resulting in the voltage gain of 37.4 . 


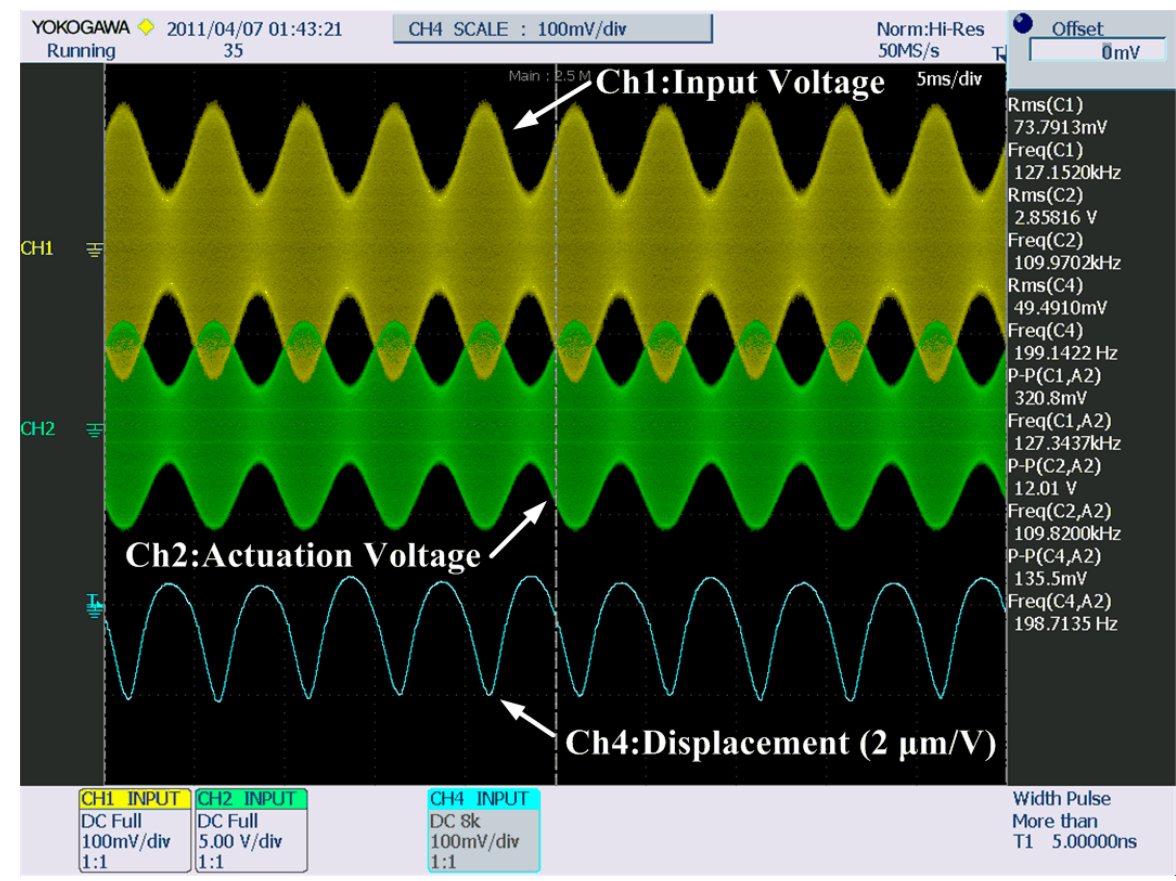

Figure 5.15 Measured displacement of the microplate driven by the resonant drive circuit: the input voltage is $321 \mathrm{mV}_{\mathrm{pp}}$, the actuation voltage amplified by the resonant drive circuit is $12 \mathrm{~V}_{\mathrm{pp}}$, and the maximum displacement of the microplate is $271 \mu \mathrm{m}$.

\subsection{Summary}

In this chapter, we present the numerical results of the cantilever beam and the fixed-fixed microbeam, when they are driven by conventional voltage control and the resonant drive circuit. The numerical results clearly show that the resonant drive circuit is able to extend their operation range at much lower input voltage. Moreover, the experiment results on the microplate supported by two identical microbeams verify that the resonant drive circuit requires much less voltage to actuate the microplate described in Section 5.3. 


\section{Chapter 6 \\ Micromirror Coupled with Resonant Drive Circuits}

A micromirror driven by conventional voltage control requires high actuation voltage supplied by a high voltage amplifier and suffers from the pull-in phenomenon that limits an operation range of a micromirror to $44 \%$ of its maximum angular displacement. To provide practical solutions to this high actuation voltage and a limited operation range, we present complete analytical and numerical models of a micromirror coupled with resonant drive circuits that enable us to actuate a micromirror at much lower supply voltage than that of conventional voltage control circuits. The presented work also facilitates the stability analysis of a micromirror coupled with a resonant drive circuit and provides how a parasitic capacitance of a micromirror and a quality factor of a resonant drive circuit affect an operation range of a micromirror. Furthermore, we present a new method of an angular displacement measurement of a micromirror by sensing the phase delay of an actuation voltage with reference to an input voltage. This new measurement method allows us to easily implement feedback control into existent systems employing a micromirror without any modification or alteration to a micromirror itself. Hence, this work presents the feasibility of a micromirror in various optical applications, in which the advantages of miniaturization, low supply voltage, and low power consumption are greatly appreciated, compared to conventional galvanometer scanners.

This chapter is organized as follows. In Section 6.1, we present the analytical model of the micromirror driven by conventional voltage control circuit and determine the fixed points of the micromirror. In Section 6.2, we derive the analytical model of the micromirror coupled with the unbalanced and balanced resonant drive circuits presented in Chapter 4 to determine its fixed points. In Section 6.3, we demonstrate the new angular displacement measurement method which is presented in Chapter 3, when the micromirror is driven by the resonant drive circuit. We discuss how the phase delay of the actuation voltage with respect to the input voltage is related with the angular displacement of the micromirror. In Section 6.4, we present FEM of the micromirror and its numerical results to compare with the analytical results. Finally, the experiment results of the micromirror with sidewall electrodes are presented in Section 6.5.

\subsection{Analytical Model of Micromirror Driven by Voltage Control}

In this section, we derive the analytical model of the micromirror presented in Figure 6.1, which is suspended by a pair of serpentine springs and is driven by conventional voltage control. We determine the fixed points of the micromirror by using its analytical model when it is driven by conventional voltage control.

First, we define the geometric properties of the micromirror to facilitate the derivation of its analytical model. In this analysis, the micromirror is regarded as a rigid body, and the serpentine torsion bars in Figure 6.2 is assumed to have such large bending stiffness in the $\mathrm{x}$ and $\mathrm{y}$ axes that the micromirror does not experience any displacement in both $\mathrm{x}$ and $\mathrm{y}$ axes during the rotation about the $\mathrm{z}$ axis. Since a serpentine torsion bar can be easily modified and fabricated to have any desirable torsional stiffness without affecting its bending stiffness $[47,48]$, these assumptions are verified by the finite element model of the micromirror and serpentine torsion bars created in COMSOL. 
As presented in Figure 6.1, the geometric properties of the micromirror are as follows: the original gap between the micromirror and its bottom electrodes is denoted by $\mathbf{d}$; the angular displacement of the micromirror is denoted by $\boldsymbol{\theta}$; and $\mathbf{l}, \mathbf{w}$, and th represent the length, the width, and the thickness of the micromirror, respectively. Because the micromirror itself is considered as a lumped rigid body, and its displacements in both $\mathrm{x}$ and $\mathrm{y}$ axes are negligible, its governing equation is simplified into an ordinary differential equation presented as thus:

$$
\mathrm{J} \frac{d^{2} \theta}{d t^{2}}+b \frac{d \theta}{d t}+k \theta=T_{e s}
$$

where $\mathbf{J}$ represents the moment of the inertia of the micromirror about the $\mathrm{z}$ axis; $\mathbf{b}$ denotes the damping coefficient representing the squeeze-film damping; $\mathbf{k}$ represents the torsional stiffness; $\boldsymbol{\theta}$ denotes the angular displacement of the micromirror; and $\mathbf{T}_{\mathbf{e s}}$ represents the electrostatic torque created by the potential difference between the micromirror and its bottom electrode.

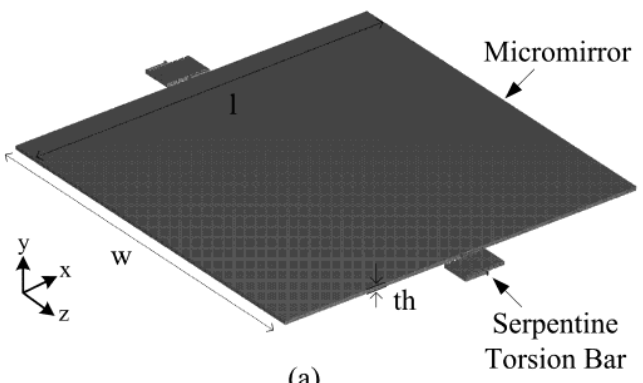

(a)

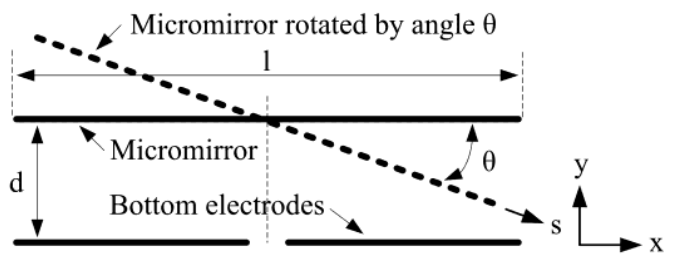

(b)

Figure 6.1 (a) Isometric view of the micromirror suspended by a pair of serpentine torsion bars and (b) its cross-sectional view in the $x-y$ plane.

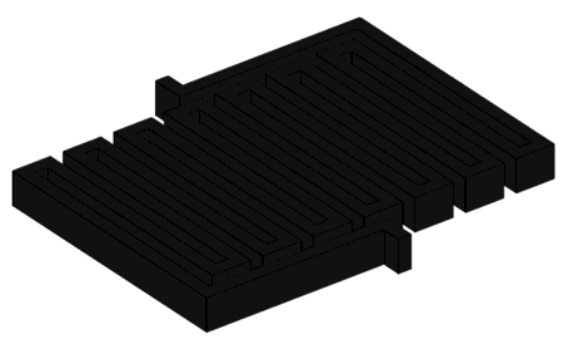

(a)

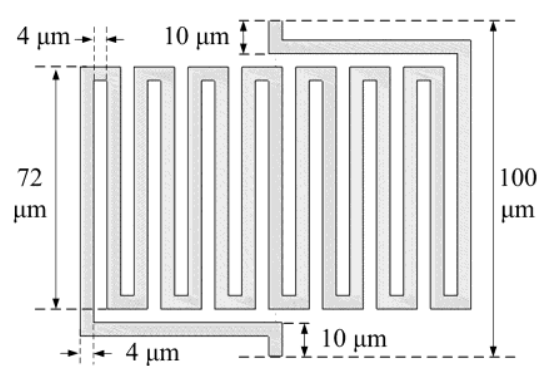

(b)

Figure 6.2 (a) Isometric view of the serpentine torsion bar made of PolySi and (b) its top view: its thickness is same as that of the micromirror, $10 \mu \mathrm{m}$. 
In order to derive the electrostatic torque exerted on the micromirror, we must solve the partial differential equation defined by the Gauss's law for electric field. However, we simplify the derivation of the electrostatic torque by using the small angle approximation [49].

$$
\sin \theta \simeq \theta, \tan \theta \simeq \theta \text {, if } \theta \ll 1
$$

Given the geometric configuration in Figure 6.1-(b), the maximum angular displacement $\boldsymbol{\theta}_{\max }$ is derived, and the normalized angular displacement $\boldsymbol{\varphi}$ is defined in terms of $\boldsymbol{\theta}$ and $\boldsymbol{\theta}_{\max }$ as thus:

$$
\sin \theta_{\max }=\frac{d}{l / 2}=\frac{2 d}{l}, \varphi \doteq \frac{\sin \theta}{\sin \theta_{\max }}=\frac{l}{2 d} \theta
$$

Since the displacements of the serpentine torsion bars in both $\mathrm{x}$ and $\mathrm{y}$ axes are considered negligible, the electrostatic torque $\mathbf{T}_{\text {es }}$ created by the electrostatic force between the micromirror and its bottom electrode is derived by using the parallel-plate capacitor theorem and the small angle approximation [49]. The electrostatic force exerting on an infinitesimal segment of the micromirror and its bottom electrode is derived as thus:

$$
d F_{e s}=\frac{1}{2} \frac{\varepsilon w d s V^{2}}{(d-s \sin \theta)^{2}}
$$

where $\mathbf{F}_{\mathbf{e s}}$ denotes the electrostatic force; $\boldsymbol{\varepsilon}$ stands for the permittivity of the air; and $\mathbf{s}$ represents the local coordinate attached to the rotational center of the micromirror.

Then, we obtain the electrostatic torque by integrating the electrostatic force over an half length of the micromirror as follows:

$$
\begin{aligned}
T_{e s} & =\int_{0}^{\frac{l}{2}} s \cos \theta d F_{e s}=\frac{\varepsilon w \cos \theta V^{2}}{2} \int_{0}^{\frac{l}{2}} \frac{s}{(d-s \sin \theta)^{2}} d s \\
& =\frac{\varepsilon w \cos \theta}{2 \sin ^{2} \theta}\left(\ln \left(1-\frac{l}{2 d} \theta\right)+\frac{\frac{l}{2 d} \theta}{1-\frac{l}{2 d} \theta}\right) V^{2} \\
& =\frac{1}{8} \frac{\varepsilon w l^{2}}{d^{2} \varphi^{2}}\left(\frac{\varphi}{1-\varphi}+\ln (1-\varphi)\right) V^{2}
\end{aligned}
$$

where $\mathbf{V}$ represents the potential difference between the micromirror and its bottom electrode.

After substituting $\mathbf{T}_{\text {es }}$ in Eq. 6.5 into Eq. 6.1, we further simplify Eq. 6.1 in terms of the normalized angular displacement $\varphi$ as thus:

$$
\mathrm{J} \frac{d^{2} \varphi}{d t^{2}}+b \frac{d \varphi}{d t}+k \varphi=\frac{1}{16} \frac{\varepsilon w l^{3}}{d^{3} \varphi^{2}}\left(\frac{\varphi}{1-\varphi}+\ln (1-\varphi)\right) V^{2}
$$

Using the pull-in voltage of the micromirror, derived by Sattler et al. [49] in Eq. 6.7 and the natural frequency of the micromirror, we render Eq. 6.6 into the non-dimensional governing equation shown in Eq. 6.8.

$$
V=V_{p i} V_{n}=\sqrt{6.616 \frac{k d^{3}}{\varepsilon w l^{3}}} V_{n}
$$


where $\mathbf{V}_{\mathbf{p i}}$ represents the pull-in voltage of the micromirror; and $\mathbf{V}_{\mathbf{n}}$ denotes the normalized input voltage in terms of $\mathbf{V}_{\mathbf{p i}}$.

After the normalization, we obtain the governing equation in a non-dimensional form as thus:

$$
\ddot{\varphi}+2 \xi \omega_{m} \dot{\varphi}+\omega_{m}^{2} \varphi=0.4135 \frac{\omega_{m}^{2}}{\varphi^{2}}\left(\frac{\varphi}{1-\varphi}+\ln (1-\varphi)\right) V_{n}{ }^{2}
$$

where $\xi$ denotes the damping ratio; and $\omega_{\mathrm{m}}$ represents the mechanical resonant frequency of the micromirror in the torsional mode.

In order to determine the fixed points of the micromirror, we solve Eq. 6.8 in terms of the normalized angular displacement and the normalized input voltage. Since the angular velocity and acceleration are zero at the fixed points, Eq. 6.8 is further simplified into Eq. 6.9 as thus:

$$
T_{n e t}=-\varphi+\frac{0.4135}{\varphi^{2}}\left(\frac{\varphi}{1-\varphi}+\ln (1-\varphi)\right) V_{n}^{2}
$$

where $\mathbf{T}_{\text {net }}$ represents the sum of the mechanical restoring torque and the electrostatic torque.

Since the micromirror reaches the equilibrium when the mechanical restoring torque and the electrostatic torque are equal, but in opposite direction, we determine the fixed points $\varphi^{*}$ of the micromirror by setting $\mathbf{T}_{\text {net }}$ to zero in Eq. 6.9 and solving it.

Next, we evaluate the stability of the fixed points by computing the derivation of $\mathbf{T}_{\text {net }}$ with respect to the normalized angular displacement $\varphi$ at the fixed points $\varphi^{*}$, which are presented in Figure 6.3. The pull-in point, where the saddle-node bifurcation occurs, is also determined through the evaluation of the derivative of $\mathbf{T}_{\text {net }}$; the pull-in occurs at $44 \%$ of the maximum angular displacement: $\boldsymbol{\varphi}=0.44$.

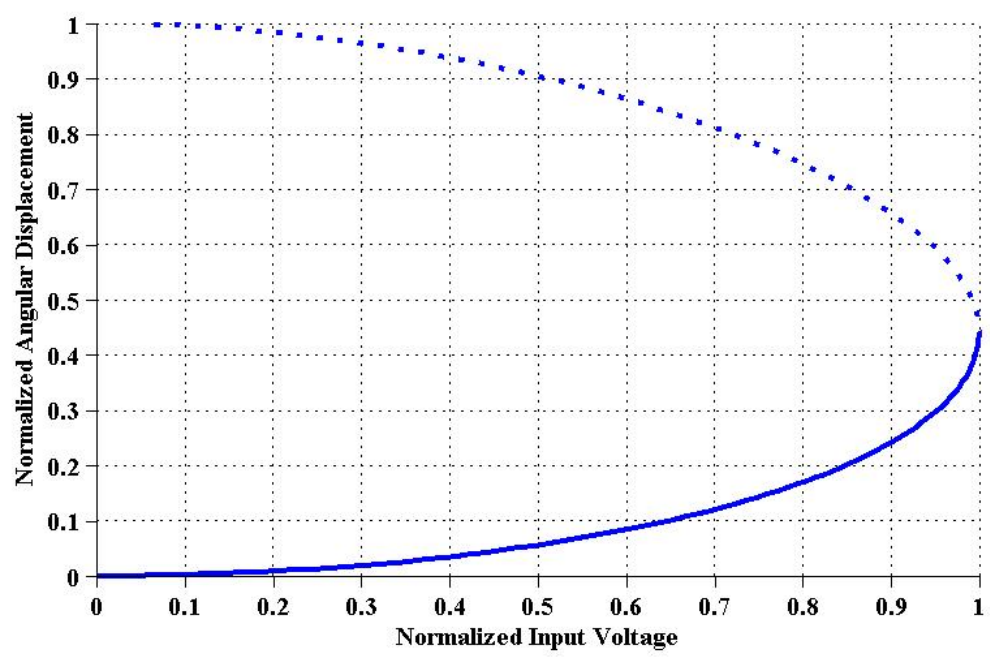

Figure 6.3 Fixed points of the micromirror driven by voltage control; the solid line represents the stable fixed points, and the dotted line denotes the unstable fixed points of the micromirror; and the saddle-node bifurcation occurs at $\varphi=0.44$. 


\subsection{Analytical Model of Micromirror Coupled with Resonant Drive Circuits}

As presented in [50], the resonant drive circuits are capable of driving the micromirror at a low supply voltage. The simplified schematics of the unbalanced and balanced resonant drive circuits are presented in Figure 6.4-(a) and (b), respectively.

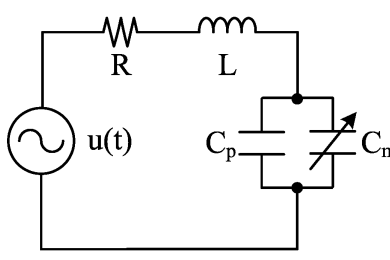

(a)

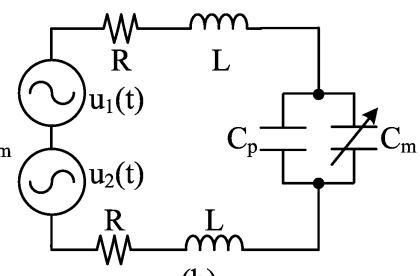

(b)

Figure 6.4 (a) Schematics of an unbalanced resonant drive circuit and (b) a balanced resonant drive circuit: $\mathbf{C}_{\mathbf{m}}$ represents the capacitance of the micromirror; $\mathbf{C}_{\mathbf{p}}$ denotes the parasitic capacitance; $\mathbf{R}$ represents the parasitic resistance of the resonant drive circuit; $\mathbf{L}$ denotes the inductance of the inductor; and $\mathbf{u}_{1}(\mathbf{t})$ and $\mathbf{u}_{2}(\mathbf{t})$ represent two AC voltage sources being $180^{\circ}$ out of phase to each other.

Although the balanced resonant drive circuit has a lower quality factor than that of the unbalanced resonant drive circuit, the balanced resonant drive circuit has a higher voltage gain at a lower resonant frequency, which leads to the lower power consumption. The schematic of the balanced resonant drive circuit, which is implemented with two operational amplifiers arranged in the bridge-tied load (BTL) configuration, is shown in Figure 6.5.

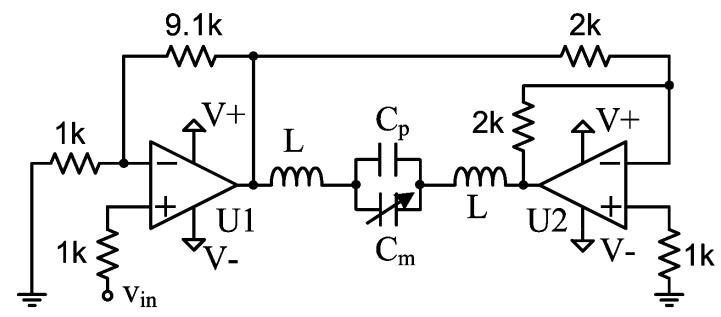

Figure 6.5 Two op-amps in the BTL configuration: U1 is configured as a non-inverting amplifier with the voltage gain of 10.1 , and U2 is arranged to be an inverting buffer to provide $180^{\circ}$ phase shift with respect to the output of $U 1$.

The governing equations of the micromirror coupled with the unbalanced resonant drive circuits presented in Figure 6.4-(a) are derived and presented as thus:

$$
\left\{\begin{array}{c}
J \ddot{\varphi}+b \dot{\varphi}+k \varphi=\frac{1}{16} \frac{\varepsilon w l^{3}}{d^{3} \varphi^{2}}\left(\frac{\varphi}{1-\varphi}+\ln (1-\varphi)\right) V^{2} \\
L\left(C_{m}+C_{p}\right) \ddot{V}+R\left(C_{m}+C_{p}\right) \dot{V}+V=u(t)
\end{array}\right.
$$

The governing equations of the micromirror coupled with the balanced resonant drive circuits presented in Figure 6.4-(b) are also derived and presented as follows: 


$$
\left\{\begin{array}{c}
J \ddot{\varphi}+b \dot{\varphi}+k \varphi=\frac{1}{16} \frac{\varepsilon w l^{3}}{d^{3} \varphi^{2}}\left(\frac{\varphi}{1-\varphi}+\ln (1-\varphi)\right) V^{2} \\
2 L\left(C_{m}+C_{p}\right) \ddot{V}+2 R\left(C_{m}+C_{p}\right) \dot{V}+V=2 u(t)
\end{array}\right.
$$

The initial resonant frequency and the initial quality factor of both resonant drive circuits are derived and presented as thus:

$$
\begin{aligned}
& \omega_{0,1}=\frac{1}{\sqrt{L C_{0}(r+1)}}, \quad Q_{f, 1}=\sqrt{\frac{L}{R^{2} C_{0}(r+1)}} \\
& \omega_{0,2}=\frac{1}{\sqrt{2 L C_{0}(r+1)}}, \quad Q_{f, 2}=\sqrt{\frac{L}{2 R^{2} C_{0}(r+1)}}
\end{aligned}
$$

where $\mathbf{C}_{\mathbf{0}}$ represents the initial capacitance of the micromirror at rest; $\mathbf{r}$ stands for the ratio of $\mathbf{C}_{\mathbf{p}}$ to $\mathbf{C}_{0} ; \boldsymbol{\omega}_{0,1}$ and $\boldsymbol{\omega}_{0,2}$ denote the initial resonant frequency of the unbalanced and the balanced resonant drive circuits, respectively; and $\mathbf{Q}_{\mathrm{f}, 1}$ and $\mathbf{Q}_{\mathrm{f}, 2}$ represent the initial quality factor of the unbalanced and the balanced resonant drive circuits, respectively.

As presented in [50], the governing equations of both resonant drive circuits presented in Eq. 6.10 and 6.11 are linear with the time-varying parameter $\mathbf{C}_{\mathbf{m}}$ that varies slowly with respect to the input signal. In other words, a very large number of cycles of the input signal elapses by the time there is any significant change in the angular displacement or the capacitance of the micromirror. Hence the capacitance of the micromirror $\mathbf{C}_{\mathbf{m}}$ can be regarded as a constant over a short period of time from the perspective of the resonant drive circuits. Thus, the resonant drive circuits can be considered as a linear time-invariant system and transformed into algebraic equations through the Laplace transformation. The frequency response of both the resonant drive circuits at their initial resonant frequencies is presented in Eq. 6.13:

$$
\left.H(j \omega)\right|_{\omega=\omega_{0}}=\frac{Q_{f} C_{0}(r+1)}{Q_{f}\left(C_{0}-C_{m}\right)+j\left(C_{p}+C_{m}\right)}
$$

where the capacitance of the micromirror $\mathbf{C}_{\mathbf{m}}$ is derived from the geometric parameters in terms of the normalized angular displacement $\boldsymbol{\varphi}$ and $\mathbf{C}_{\mathbf{0}}$ as thus:

$$
C_{m}=\int_{0}^{\frac{l}{2}} \frac{\varepsilon w d s}{d-s \sin \theta}=-\frac{\varepsilon w l}{2 d} \frac{\ln (1-\varphi)}{\varphi}=-C_{0} \frac{\ln (1-\varphi)}{\varphi}
$$

Then, the voltage gain at their initial resonant frequencies is derived from the frequency response of Eq. 6.13 in terms of the normalized angular displacement $\varphi$ as follows:

$$
\left|H\left(j \omega_{0}\right)\right|=\frac{Q_{f}(r+1) \varphi}{\sqrt{Q_{f}^{2}(\varphi+\ln (1-\varphi))^{2}+(r \varphi-\ln (1-\varphi))^{2}}}
$$

Since the electrical resonant frequency $\omega_{0}$ of the resonant drive circuits is much higher than the mechanical natural frequency $\omega_{\mathrm{m}}$ of the micromirror, the micromirror only responds to the RMS value $\mathbf{V}_{\text {rms }}$ of the actuation voltage, which are presented as thus: 


$$
\begin{aligned}
& V_{r m s, 1}=\frac{1}{\sqrt{2}}\left|H\left(j \omega_{0,1}\right)\right| u \\
& V_{r m s, 2}=\frac{1}{\sqrt{2}}\left|H\left(j \omega_{0,2}\right)\right| 2 u
\end{aligned}
$$

where $\mathbf{u}$ represents the peak voltage of the input signal.

Using the pull-in voltage presented in Eq. 6.7, we normalize the governing equation of the micromirror coupled with the unbalanced resonant drive circuit as shown in Eq.6.10 into Eq. 6.17:

$$
\begin{gathered}
\ddot{\varphi}+2 \xi \omega_{m} \dot{\varphi}+\omega_{m}^{2} \varphi= \\
0.207 \omega_{m}^{2}\left(\frac{\varphi}{1-\varphi}+\ln (1-\varphi)\right)\left(\frac{Q_{f}^{2}(r+1)^{2} \bar{u}^{2}}{Q_{f}^{2}(\varphi+\ln (1-\varphi))^{2}+(r \varphi-\ln (1-\varphi))^{2}}\right)
\end{gathered}
$$

where $\overline{\mathbf{u}}$ represents the normalized input voltage with respect to the conventional pull-in voltage $\mathbf{V}_{\mathbf{p} \mathbf{i}}$. Using the same pull-in voltage, we normalize the governing equation of the micromirror coupled with the balanced resonant drive circuit presented in Eq. 6.11 into Eq. 6.18:

$$
\begin{gathered}
\ddot{\varphi}+2 \xi \omega_{m} \dot{\varphi}+\omega_{m}^{2} \varphi= \\
0.827 \omega_{m}^{2}\left(\frac{\varphi}{1-\varphi}+\ln (1-\varphi)\right)\left(\frac{Q_{f}^{2}(r+1)^{2} \bar{u}^{2}}{Q_{f}^{2}(\varphi+\ln (1-\varphi))^{2}+(r \varphi-\ln (1-\varphi))^{2}}\right)
\end{gathered}
$$

By solving the normalized, non-dimensional governing equations presented in Eq. 6.17 and 6.18, we determine the fixed points of the micromirror coupled with the resonant drive circuits in the presence of various parasitic capacitances and evaluate the stability of the fixed points, which are presented in Figure 6.6.

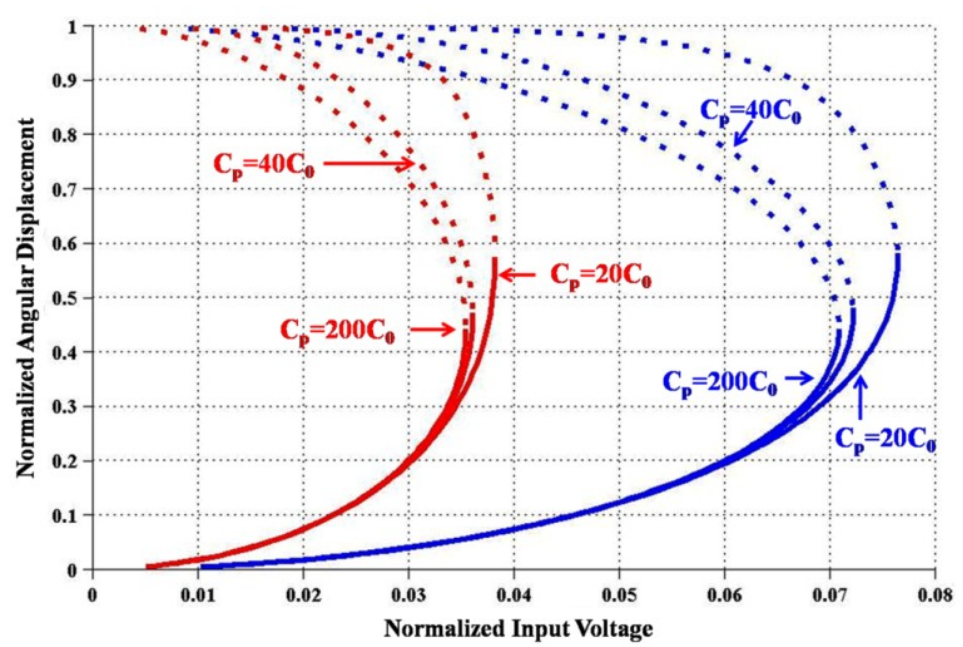

Figure 6.6 Fixed points of the micromirror driven by the resonant drive circuits with the quality factor $\mathbf{Q}_{\mathbf{f}}$ of 20: the dotted lines represent the unstable fixed points; the solid lines represent the stable fixed points; and the red lines and the blue lines denote the fixed points of the micromirror coupled with the balanced and unbalanced resonant drive circuits, respectively. 
As presented in the Figure 6.6, both resonant drive circuits are capable of driving the micromirror beyond the conventional pull-in point $\left(\varphi_{\mathbf{p i}}=0.44\right)$ in the presence of high parasitic capacitance and driving the micromirror at much lower input voltage than a conventional voltage control circuit does.

\subsection{Angular Displacement Measurement by Sensing Phase Delay between Actuation Voltage and Input Voltage}

One of the disadvantages of an electrostatic actuator is that it is difficult to measure its displacement because of its small size and the limitation of its fabrication methods. In this section, we present a new method of the angular displacement measurement by sensing the phase change of the actuation voltage with respect to the AC input voltage using a phase detector, whose simplified schematic is presented in Figure 6.7. The input signal, the frequency of which is equal to the initial electrical resonance $\omega_{0}$ of the resonant drive circuit, can be considered as an unmodulated carrier signal. As the micromirror displaces toward its bottom electrode, its capacitance increases, and the electrical resonance of the resonant drive circuits decreases. This deviation in the resonant frequency causes less voltage gain and more phase delay in the actuation voltage. In other words, the micromirror modulates the input signal with its displacement, resulting in the actuation voltage being modulated by the angular displacement of the micromirror. By demodulating the actuation voltage in terms of the voltage gain or the phase delay, we are capable of measuring the change in its capacitance in order to determine the angular displacement of the micromirror coupled with the resonant drive circuits. The voltage gain is usually measured by an envelope detector, and the phase delay of the actuation voltage is detected by mixing the input signal with the actuation voltage, which is accomplished by a phase detector that is, in essence, a frequency mixer. It turns out that the phase delay is more sensitive to the angular displacement of the micromirror than the voltage gain. Furthermore, the phase delay provides one-to-one mapping to the angular displacement without any ambiguity while the voltage gain does not, even in the presence of low parasitic capacitance.

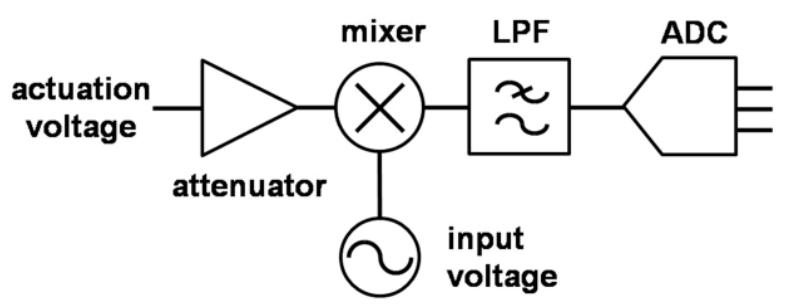

Figure 6.7 Diagram of a phase detector to measure the phase delay of the actuation voltage with respect to the input voltage.

The phase delay of the actuation voltage in terms of the normalized angular displacement is derived from the frequency response in Eq. 6.13 and is presented in Eq. 6.19:

$$
\angle H\left(j \omega_{0}\right)=-\tan ^{-1}\left(\frac{r \varphi-\ln (1-\varphi)}{Q_{f}(\varphi+\ln (1-\varphi))}\right)
$$


As indicated in Eq. 6.19, the phase delay of the actuation voltage depends solely on the angular displacement of the micromirror; the initial quality factor $\mathbf{Q}_{\mathrm{f}}$ and the ratio $\mathbf{r}$ of $\mathbf{C}_{\mathbf{p}}$ to $\mathbf{C}_{\mathbf{0}}$ are regarded as a constant, being determined through the parameter identification. Hence, we are able to determine the angular displacement of the micromirror coupled with the resonant drive circuits by measuring the phase delay of the actuation voltage with respect to the input signal, and its analytical results with various parasitic capacitances are presented in Figure 6.8.

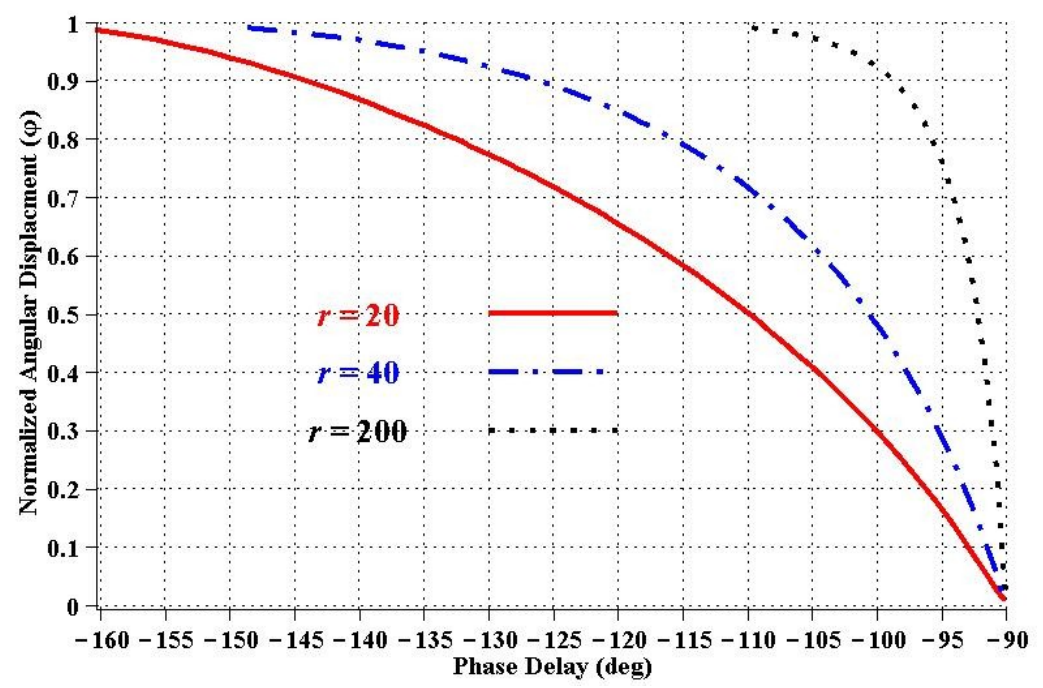

Figure 6.8 Normalized angular displacement of the micromirror driven by the resonant drive circuits with $\mathbf{Q}_{\mathrm{f}}=20$ in terms of the phase delay of the actuation voltage: the red solid line, the blue

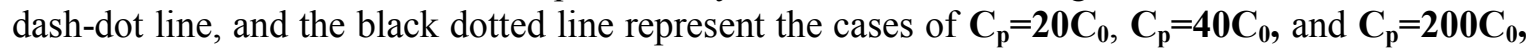
respectively.

\subsection{Finite Element Model of Micromirror}

In order to validate the analytical model of the micromirror coupled with the resonant drive circuits, we create its finite element model and implement the resonant drive circuit as an algebraic constraint on the bottom of the micromirror through a nonlinear coupling. Although a straight torsion bar is simple to be fabricated and modeled, its low bending stiffness allows the micromirror to displace in the $\mathrm{y}$ axis while rotating about the $\mathrm{z}$ axis, when the micromirror is actuated. Hence, we implement two identical serpentine torsion bars, presented in Figure 6.2, to increase the bending stiffness in the $y$ axis while reducing the torsional stiffness about the $\mathrm{z}$ axis at the same time. The physical and geometric properties of the micromirror and the resonant drive circuit implemented in their finite element models are presented in Table 6.1. To determine the bending stiffness and the torsional stiffness of the serpentine torsion bars, we evaluate the displacement of both the center of the micromirror and the outer edge of the micromirror in the y axis, while the micromirror is driven by conventional voltage control. As presented in Figure 6.7, the micromirror undergoes very small displacement in the $\mathrm{y}$ axis while rotating about the $\mathrm{z}$ axis as a result of high bending stiffness and low torsional stiffness of the serpentine torsion bars. For instance, the centre of the micromirror displaces about $0.585 \mu \mathrm{m}$ in the y axis, and the outer edge of the micromirror displaces $42.42 \mu \mathrm{m}$ in the same 
axis, when $125 \mathrm{~V}$ of the actuation voltage is applied on the micromirror, because the serpentine torsion bars provide the high bending stiffness in the y axis while having the low torsional stiffness about the $\mathrm{z}$ axis as mentioned. The displacement profiles of the micromirror actuated at $75 \mathrm{~V}, 100 \mathrm{~V}$, and $125 \mathrm{~V}$ are also presented in Figure 6.8 and prove that the serpentine torsion bars minimize the rectilinear displacement of the micromirror in the y axis while providing the low torsional stiffness about the $\mathrm{z}$ axis. The normal mode analysis of the micromirror with a pair of the serpentine torsion bars, presented in Figure 6.12, confirms the assumption made in Section 6.1: the 1st normal mode of the micromirror is torsional motion about the $\mathrm{z}$ axis; the 2 nd mode is rectilinear motion in the $\mathrm{z}$ axis; and the 3rd mode is rectilinear motion in the y axis.

Table 6.1 Parameters used for FEM of the micromirror coupled with the resonant drive circuit

\begin{tabular}{ccc}
\hline Parameter & Symbol & Value \\
\hline \hline Material density & $\rho$ & $2320\left[\mathrm{~kg} / \mathrm{m}^{3}\right]$ \\
\hline Torsional stiffness of the serpentine torsion bars & $\mathrm{k}$ & $22.44[\mathrm{nN} \cdot \mathrm{m} / \mathrm{rad}]$ \\
Length of the micromirror & 1 & $1[\mathrm{~mm}]$ \\
Width of the micromirror & $\mathrm{w}$ & $1[\mathrm{~mm}]$ \\
Thickness of the micromirror & th & $10[\mu \mathrm{m}]$ \\
Moment of inertia of the micromirror about the z axis & $\mathrm{J}$ & $1.93 \mathrm{e}-15\left[\mathrm{~kg} / \mathrm{m}^{2}\right]$ \\
Gap & $\mathrm{d}$ & $100[\mu \mathrm{m}]$ \\
Max. angular displacement & $\theta_{\mathrm{max}}$ & $11.3\left[{ }^{\circ}\right]$ \\
Conventional pull-in voltage & $\mathrm{V}_{\mathrm{pi}}$ & $129.5[\mathrm{~V}]$ \\
Normalized pull-in angle & $\varphi_{\mathrm{pi}}$ & 0.44 \\
Initial capacitance & $\mathrm{C}_{0}$ & $44.27[\mathrm{fF}]$ \\
Ratio of $\mathrm{C}_{\mathrm{p}}$ to $\mathrm{C}_{0}$ & $\mathrm{r}$ & $20,40,200$ \\
\hline Initial quality factor of the resonant drive circuit & $\mathrm{Q}_{\mathrm{f}}$ & 20 \\
Natural frequency of the micromirror & $\mathrm{f}_{\mathrm{m}}$ & $543[\mathrm{~Hz}]$ \\
\hline \hline
\end{tabular}

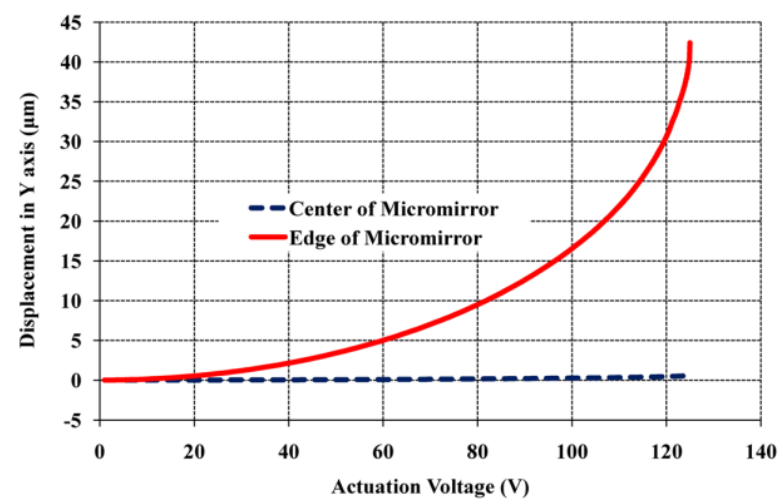

Figure 6.9 Displacement of the centre and the edge of the micromirror in the y axis 
We also evaluate the torsional stiffness of the serpentine torsion bars by applying the predetermined torque on the micromirror. As presented in Figure 6.11, the serpentine torsion bars exhibit the stiffness hardening as the angular displacement increases. However, the torsional stiffness appears to be constant as long as the angular displacement is less than $12^{\circ}$, which is greater than the maximum angular displacement of the micromirror with the given geometry. Using these numerical results, we evaluate the torsional stiffness of the serpentine torsion bars, which appears to be $22.65[\mathrm{nN} \cdot \mathrm{m} / \mathrm{rad}]$. This torsional stiffness is also confirmed by the normal mode analysis, which evaluates the torsional stiffness to be $22.44[\mathrm{nN} \cdot \mathrm{m} / \mathrm{rad}]$. Based on this torsional stiffness and the given geometry of the micromirror, we determine the pull-in voltage and the pull-in angle by using Eq. 6.7 and Eq. 6.9, which result in that the pull-in voltage is $129.5 \mathrm{~V}$, and the pull-in angle is $4.97^{\circ}$.

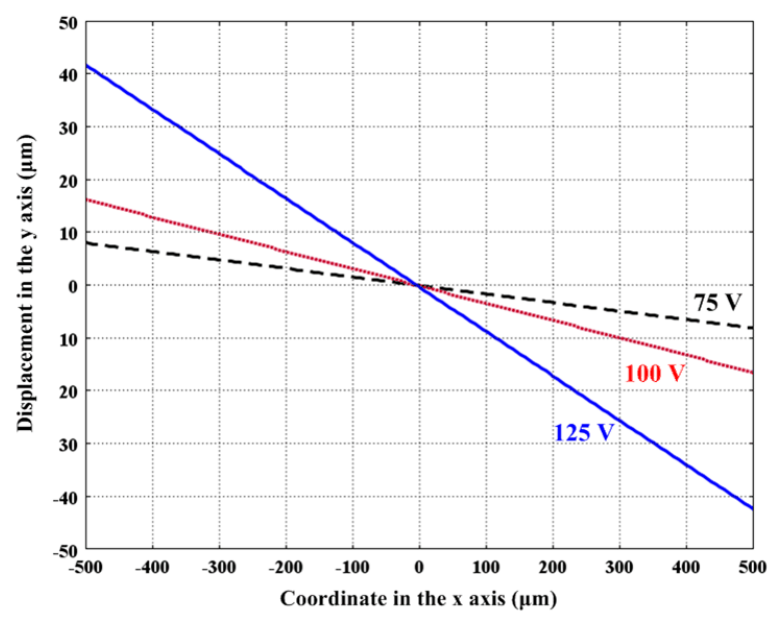

Figure 6.10 Displacement profiles of the micromirror at various actuation voltages: the dashed line, the dotted line, and the solid line represent the displacement profile of the micromirror when its actuation voltage are $75 \mathrm{~V}, 100 \mathrm{~V}$, and $125 \mathrm{~V}$, respectively.

In order to validate these analytical results, we make use of the same FEM of the micromirror supported by a pair of the serpentine torsion bars. As presented in Figure 6.13-(a), two serpentine torsion bars and the micromirror are meshed with tetrahedrons and hexahedrons, respectively. Air between the micromirror and its bottom electrodes is also meshed with hexahedrons to reduce the number of nodes. Figure 6.13-(b) presents the micromirror post-processed with the displacement in the $\mathrm{y}$ axis, when $125 \mathrm{~V}$ of the actuation voltage is applied to the micromirror. The finite element analysis indicates that the pull-in voltage is $125.1 \mathrm{~V}$, which is less than the pull-in voltage obtained from the analytical model of the micromirror, and the pull-in angle is $4.78^{\circ}$, which is presented in Figure 6.14. This difference is, we believe, caused by the micromirror's fringe field and the small angle approximation we use to derive the analytical model of the micromirror.

The normal mode analysis is also performed to determine a set of the natural frequencies and mode shapes of the micromirror supported by two serpentine torsion bars, and the first six natural frequencies and mode shapes are presented in Figure 6.12. 


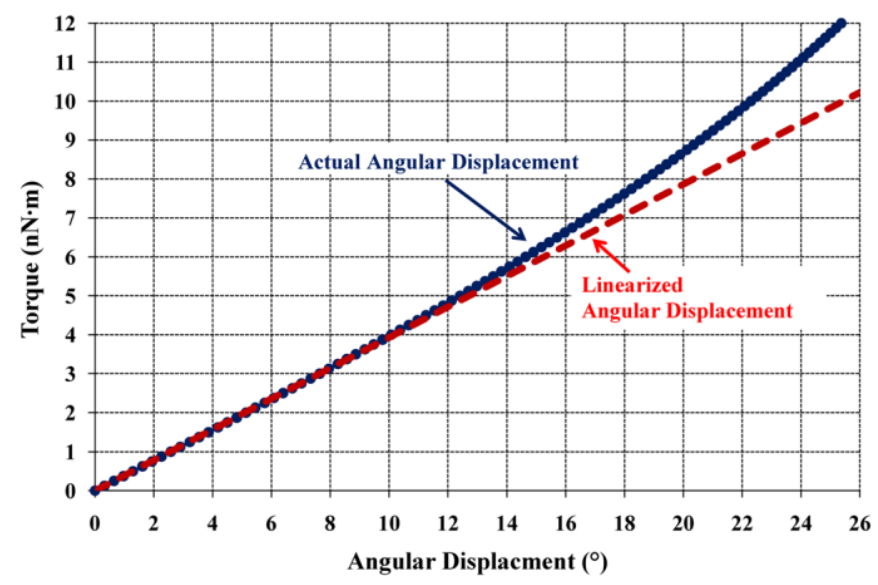

Figure 6.11 Torsional stiffness of a pair of the serpentine torsion bars about the $\mathrm{z}$ axis and their linearized torsional stiffness.

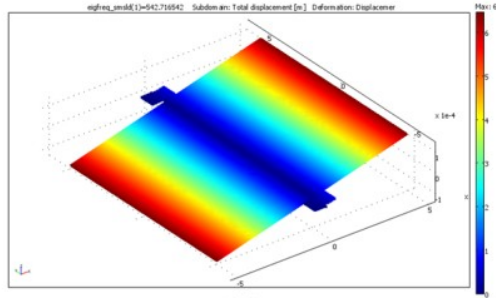

(a)

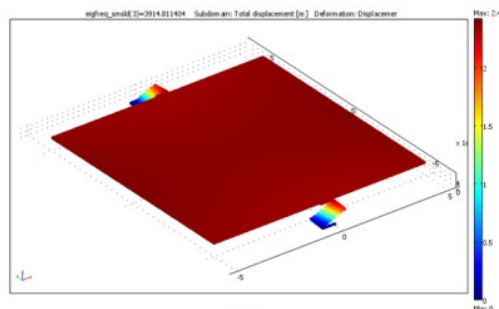

(c)

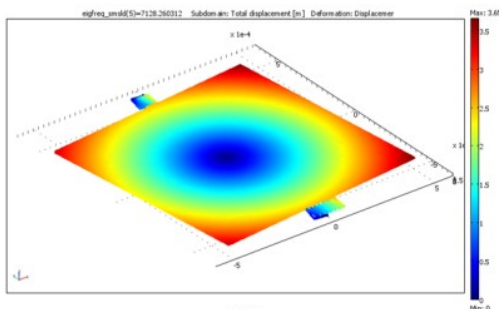

(e)

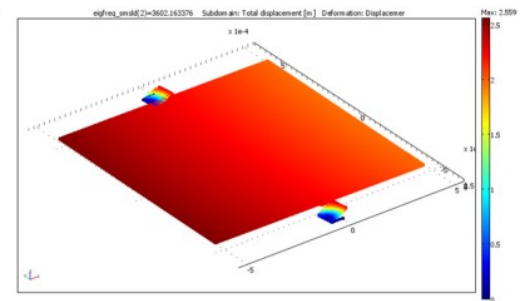

(b)

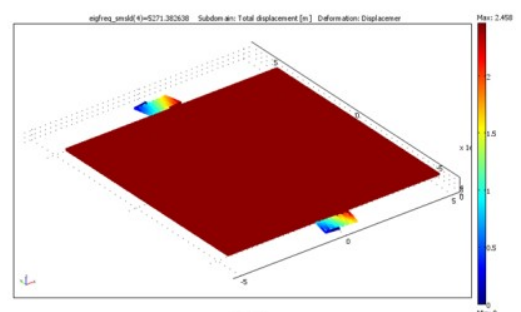

(d)

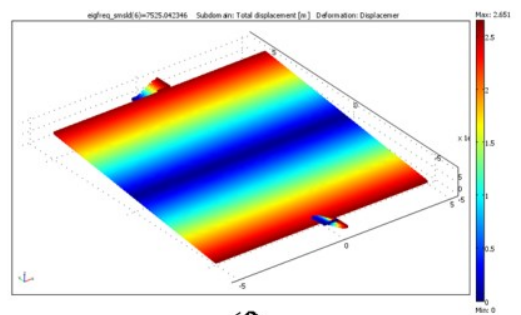

(f)

Figure 6.12 Normal mode analysis of the micromirror suspended by a pair of the serpentine springs: (a) the $1^{\text {st }}$ mode at $543 \mathrm{~Hz}$; (b) the $2^{\text {nd }}$ mode at $3602 \mathrm{~Hz}$; (c) the $3^{\text {rd }}$ mode at $3915 \mathrm{~Hz}$; (d) the $4^{\text {th }}$ mode at $5271 \mathrm{~Hz}$; (e) the $5^{\text {th }}$ mode at $7218 \mathrm{~Hz}$; (f) the $6^{\text {th }}$ mode at $7525 \mathrm{~Hz}$. 
The same FEM of the micromirror with a pair of the serpentine torsion bars is used to determine the fixed points of the micromirror that is actuated by the unbalanced resonant drive circuit, which is implemented as an algebraic constraint on a boundary condition in its FEM.

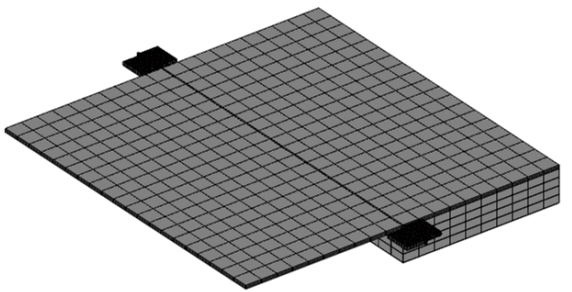

(a)

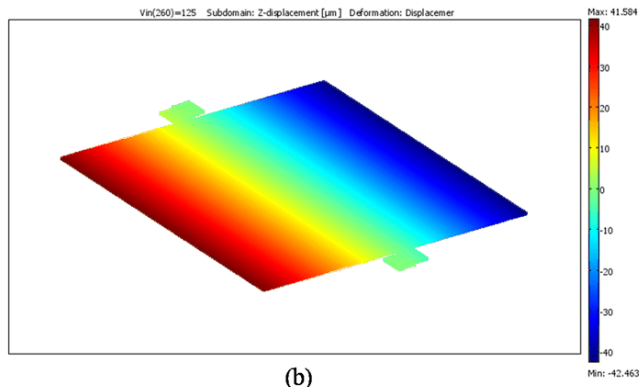

Figure 6.13 (a) the micromirror meshed with hexahedral elements and (b) the micromirror postprocessed with the displacement in the y axis at the actuation voltage of $125 \mathrm{~V}$.

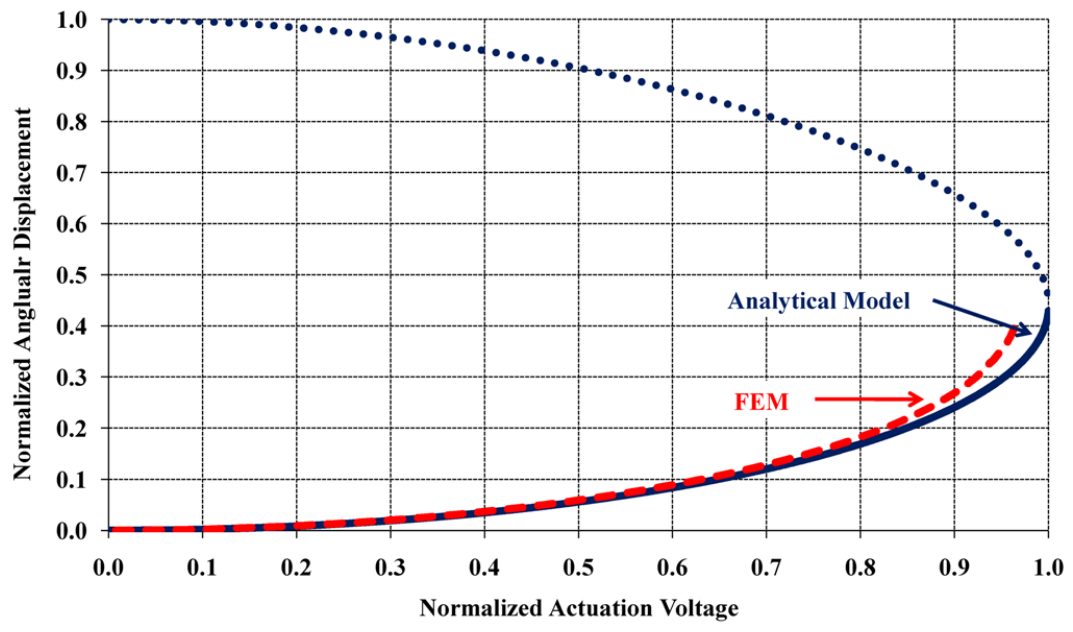

Figure 6.14 Fixed points of the micromirror with a pair of the serpentine torsion bars driven by the conventional voltage control: the dashed line represent the fixed points of the micromirror obtained through its FEM; the solid line denotes the stable fixed points of the micromirror derived from its analytical model; and the dotted line indicates the unstable fixed points. 
Using this model, we determine the fixed points of the micromirror coupled with the resonant drive circuit in three cases: $\mathbf{C}_{\mathbf{p}}=20 \mathbf{C}_{\mathbf{0}}, \mathbf{C}_{\mathbf{p}}=40 \mathbf{C}_{\mathbf{0}}$; and $\mathbf{C}_{\mathbf{p}}=200 \mathbf{C}_{\mathbf{0}}$. Figure 6.15 presents both the analytical results and numerical results for comparison. The numerical results from FEA are in good agreement with the analytical results, proving that the resonant drive circuit is able to drive the micromirror at much low input voltage and extend the operation range of the micromirror in the presence of high parasitic capacitance. The peak voltages of the input signal to the resonant drive circuit at the pull-in are $9.87 \mathrm{~V}$ at $\mathbf{C}_{\mathbf{p}}=20 \mathbf{C}_{\mathbf{0}}, 9.19 \mathrm{~V} \mathbf{C}_{\mathbf{p}}=40 \mathbf{C}_{\mathbf{0}}$, and $8.84 \mathrm{~V}$ at $\mathbf{C}_{\mathbf{p}}=200 \mathbf{C}_{\mathbf{0}}$, respectively.

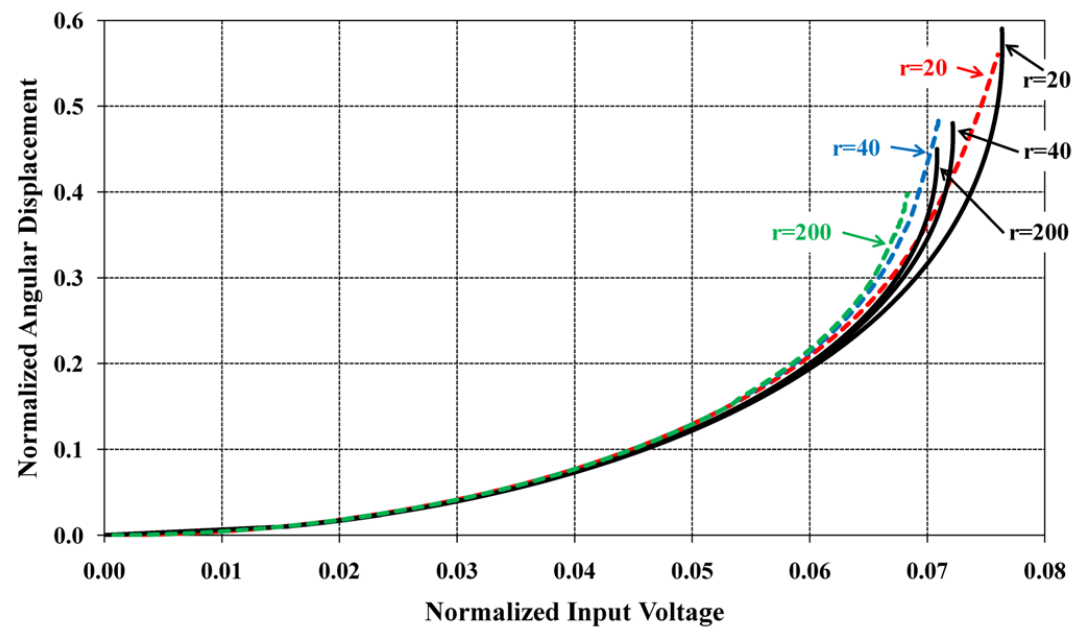

Figure 6.15 Fixed points of the micromirror driven by the unbalanced resonant drive circuit with various parasitic capacitances: the dashed lines represent the fixed points of the micromirror obtained from its FEA; and the solid black lines denote the fixed points derived from its analytical model. 


\subsection{Experiment on Micromirror with Sidewall Electrodes Driven by Resonant Drive Circuit}

To demonstrate the advantages of the resonant drive circuit mentioned in the previous sections, we perform the experiment on the micromirror with sidewall electrodes, the Scanning Electron Micrographs (SEM) of which are shown in Figure 6.16.

The micromirror with sidewall electrodes is fabricated on $100 \mathrm{~mm}$ silicon-on-insulator (SOI) wafers and patterned by hybrid surface/bulk micromachining technology. In order to fabricate the micromirror with sidewall electrodes, the fabrication process is divided into three stages: the fabrication of the micromirror plate, the fabrication of sidewall electrodes, and the assembly of the micromirror plate and sidewall electrodes. A SOI wafer can provide an accurate thickness for MEMS devices and be used to forming complicated 3D patterns on top of the device layer and the handle layer. First, the micromirror plate is fabricated on a 4 " SOI wafer, device layer $12 \mu \mathrm{m}$, buried oxide sacrificial layer $0.5 \mu \mathrm{m}$, and handle layer $290 \mu \mathrm{m}$. Layers of $0.5 \mu \mathrm{m}$ and $2 \mu \mathrm{m}$ silicon dioxide are deposited on top of the device layer and the handle layer by plasma enhanced chemical vapor deposition (PECVD), respectively. On the device layer, the micromirror plate is patterned by reactive ion etching (RIE) and deep RIE (DRIE) and the tiny serpentine torsion bars are formed. Then the back side features of the micromirror plate are etched by RIE and DRIE as well. The total thickness (35 $\mu \mathrm{m})$ of the micromirror plate is obtained. A wet etching method is adopted to release the micromirror plate by using 6:1 Buffered Oxide Etchant (BOE).

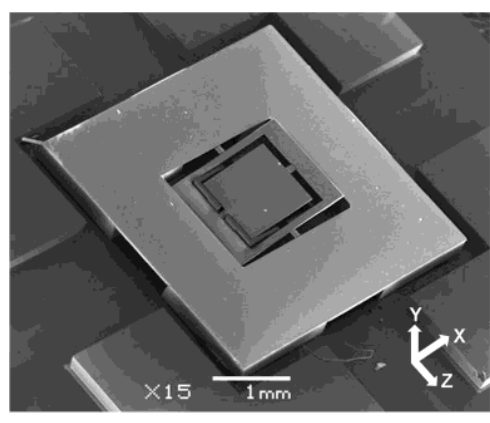

(a)

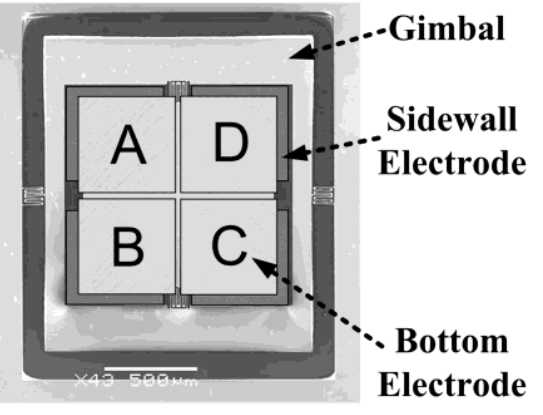

(b)

Figure 6.16 SEMS of the micromirror with sidewall electrodes: (a) the isometric view of the micromirror and (b) the top view of the micromirror showing its bottom electrodes and sidewall electrodes.

Finally, Ti/Au reflective layer is deposited on the surface of the micromirror plate in order to increase the reflective efficiency. Second, the sidewall electrodes are fabricated on another 4 "SOI wafer: device layer $250 \mu \mathrm{m}$, buried silicon dioxide sacrificial layer $2 \mu \mathrm{m}$, and handle layer $300 \mu \mathrm{m}$. Sidewall electrodes are vertical and have a high aspect ratio. RIE and DRIE are adopted to build the sidewall electrodes. Since the height of the sidewall is $250 \mu \mathrm{m}$, spinning photoresist (PR) and patterning the trace and pad on the vertical walls is impossible. So the silicon shadow mask is chosen for patterning the features. The shadow mask with alignment marks is assembly on the sidewall electrodes. The tolerance of assembly can be less than $10 \mu \mathrm{m}$. Subsequently, the sidewall electrodes are put into CVC 
Sputter Deposition System for depositing Cr/Al. The shadow mask is removed by a lift-off process. Finally, the wafer of sidewall electrodes is diced into small dies by the dicing saw. The last step is to assemble the micromirror plate and sidewall electrodes through using a transparent alignment tool built on a Pyrex glass wafer with four pins (height $50 \mu \mathrm{m}$ ) and the HTG System III-HR Contact Aligner. The assembly process resembles flip-chip method. The die of the sidewall electrodes with a small quantity of epoxy glue, SU-8, is put on top of HTG stage, and then the micromirror plate is stacked and aligned on the sidewall electrodes. Applied low pressure through the alignment tool, the micromirror plate is glued to the sidewall electrodes. Subsequently, the micromirror device is placed in an oven at $90{ }^{\circ} \mathrm{C}$ for 12 hours. In this experiment, we rotate the micromirror about the $\mathrm{z}$ axis only as shown in Figure 6.16-(a). To do that, the bottom electrodes $\mathrm{B}$ and $\mathrm{C}$ in Figure 6.16-(b) are electrically connected and the micromirror with the gimbal acts as a moveable electrode. The initial capacitance $\mathrm{C}_{0}$ of the micromirror is obtained from its finite element model and estimated to be 100 fF. Its total capacitance including the parasitic capacitance $C_{p}$ is measured to be $40 \mathrm{pF}$ by the LCR meter 889A from BK Precision. Hence the ratio of $\mathrm{C}_{\mathrm{p}}$ to $\mathrm{C}_{0}$ is 400 . The resonant drive circuit implemented with logic gates arranged in the BTL configuration is built with two $15 \mathrm{mH}$ ferrite core inductors, one MM74C240N inverting buffer, and one MM74C244N non-inverting buffer as presented in Figure 4.7. The function generator 4086 from BK Precision is employed to drive the resonant drive circuit at the switching frequency $145 \mathrm{kHz}$, which is fixed at the initial resonant frequency of the resonant drive circuit. Then, the programmable DC power supply 1788 from BK Precision is used to supply power to the resonant drive circuit. The power supply is controlled to vary its voltage from $4 \mathrm{~V}$ to $9 \mathrm{~V}$ while the mechanical angular displacement of the micromirror is measured. Later, a high voltage amplifier powered by a high voltage power supply $(300 \mathrm{~V})$ is used to actuate the same micromirror with sidewall electrodes.

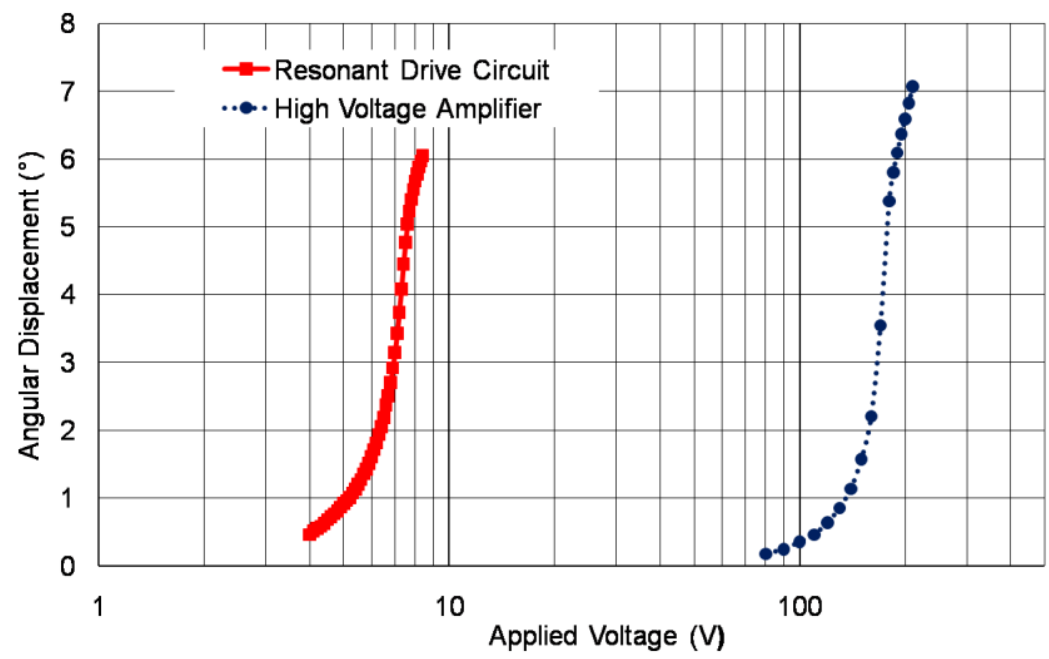

Figure 6.17 Mechanical angular displacement of the micromirror with sidewall electrodes driven by the resonant drive circuit and a high voltage amplifier. 
As presented in Figure 6.17, the high voltage amplifier rotates the micromirror by $6^{\circ}$ at $180 \mathrm{~V}$ and the resonant drive circuit rotates the micromirror by $6^{\circ}$ at $8.5 \mathrm{~V}$. The advantage of the resonant drive circuit is clearly shown in Figure 6.17 as it minimizes the voltage requirement and rotates the micromirror without a high voltage power supply and a high voltage amplifier, which normally require a heat sink to dissipate heat to the ambient air. Hence, it is much easier to implement the micromirror into a small form factor that is crucial in medical and portable applications.

\subsection{Summary}

One of the challenges that an electrostatically actuated micromirror has been struggled with is its high actuation voltage, which requires a high voltage power supply and a high voltage amplifier. Moreover the operation range of the micromirror is limited to $44 \%$ of the maximum angular displacement due to the saddle-node bifurcation, and the angular displacement measurement of the micromirror is difficult to be implemented due to its minute size and constraints imposed by its fabrication methods. To overcome these problems, we present the resonant drive circuit, which operates at much lower supply voltage, provide the complete analytical models of the micromirror coupled with the resonant drive circuit that are validated by its FEA, and present the new method of the angular displacement measurement. The presented analytical models show that the resonant drive circuit enables us to actuate the micromirror at low supply voltage and that its operation range can be extended in the presence of various parasitic capacitances. Furthermore, unlike conventional high voltage actuation circuits, the resonant drive circuit does not pose any industrial hazard related to high voltage, because there is no high voltage or high energy storage component in the circuit. Hence it is inherently robust and can be safely implemented in many industrial and medical applications where high voltage is not allowed. Finally, the new method of the angular displacement measurement of the micromirror allows a feedback control to be implemented without any modification or altercation on the micromirror itself. 


\section{Chapter 7}

\section{Feedback Control with Resonant Drive Circuit}

As described in the previous chapters, we now have a way of measuring the displacement of the electrostatic actuator by sensing the phase delay of either the actuation voltage or the input current with respect to the input voltage. In this chapter, we present two feedback controllers that use the displacement measurement to extend the operation range of the parallel-plate actuator and stabilize the parallel-plate actuator beyond its conventional pull-in point.

This chapter is organized as follows. In Section 7.1, we present the parallel-plate actuator coupled with the resonant drive circuit that is driven by a nonlinear feedback controller. We derive the governing equation of the parallel-plate actuator with the controller and determine its fixed points. In section 7.2, we present a simple but effective linear feedback controller to stabilize the parallel-plate actuator in the presence of high parasitic capacitance. We derive the governing equation of the parallel-plate actuator implemented with its controller to determine its fixed points as well.

\subsection{Nonlinear Feedback Control with Resonant Drive Circuit}

The diagram of the parallel-plate actuator coupled with the resonant drive circuit that is controlled by a nonlinear feedback controller is presented in Figure 7.1. This explicit feedback controller is used to enhance the inherent negative feedback of the resonant drive circuit so that the parallel-plate actuator can be displaced over its entire gap even in the presence of high parasitic capacitance.

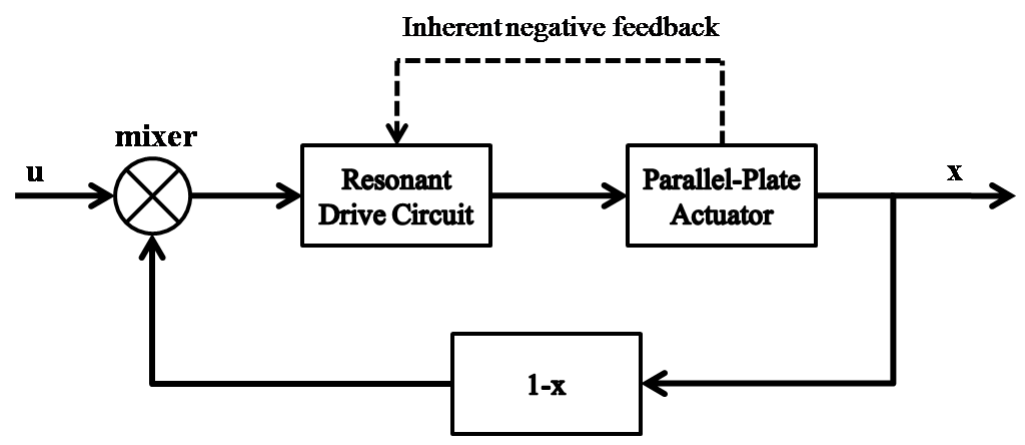

Figure 7.1 Diagram of the nonlinear feedback controller implemented with the resonant drive circuit that drives the parallel-plate actuator.

We derive the non-dimensional governing equation of the parallel-plate actuator coupled with the unbalanced resonant drive circuit, which is controlled by the nonlinear controller in Figure 7.1, as thus:

$$
\ddot{x}+2 \xi \omega_{n} \dot{x}+\omega_{m}^{2} x=\frac{2}{27} \omega_{m}^{2} \frac{Q_{f}^{2}(r+1)^{2}(1-x)^{2} \bar{u}^{2}}{\left(Q_{f}^{2} x^{2}+(r(1-x)+1)^{2}\right)}
$$


Using the same methods described in Chapter 3, we determine the fixed points of the parallel-plate actuator and their stability and present them in Figure 7.2.

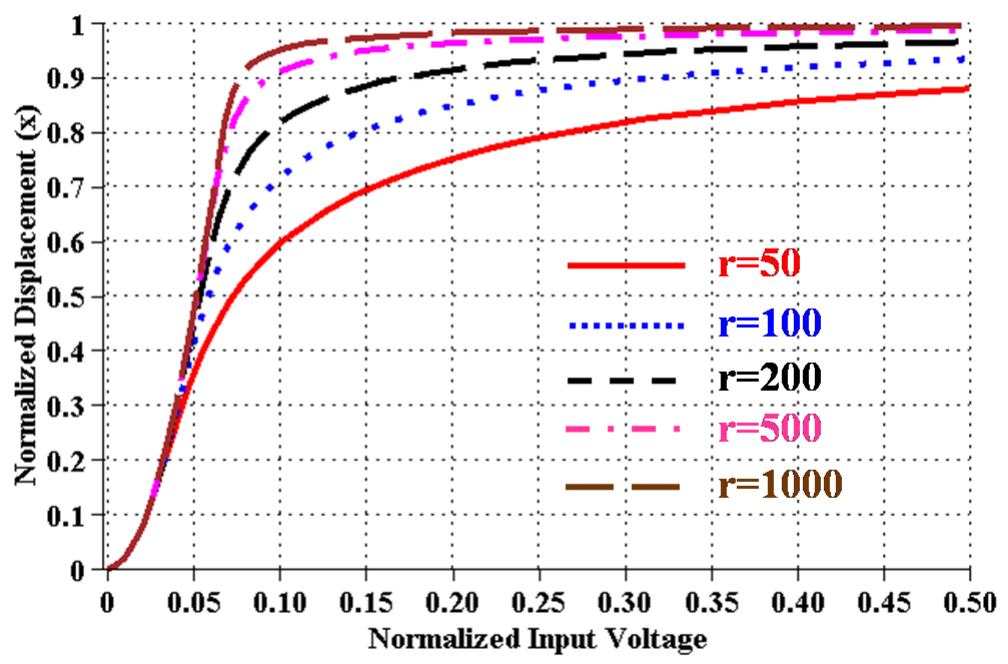

Figure 7.2 Stable fixed points of the parallel-plate actuator coupled with the resonant drive circuit and the nonlinear controller in terms of the quality factor $\mathrm{Q}_{\mathrm{f}}=50$ and various parasitic capacitance.

As presented in Figure 7.2, the parallel-plate actuator does not undergo the saddle-node bifurcation even in the presence of very high parasitic capacitance. In other words, the nonlinear feedback controller in Figure 7.1 is able to stabilize and displace the parallel-plate actuator beyond its conventional pull-in point and still requires lower input voltage than the conventional pull-in voltage.

\subsection{Linear Feedback Control with Resonant Drive Circuit}

In this section, we present the parallel-plate actuator coupled with the resonant drive circuit that is controlled by a linear feedback controller presented in Figure 7.3.

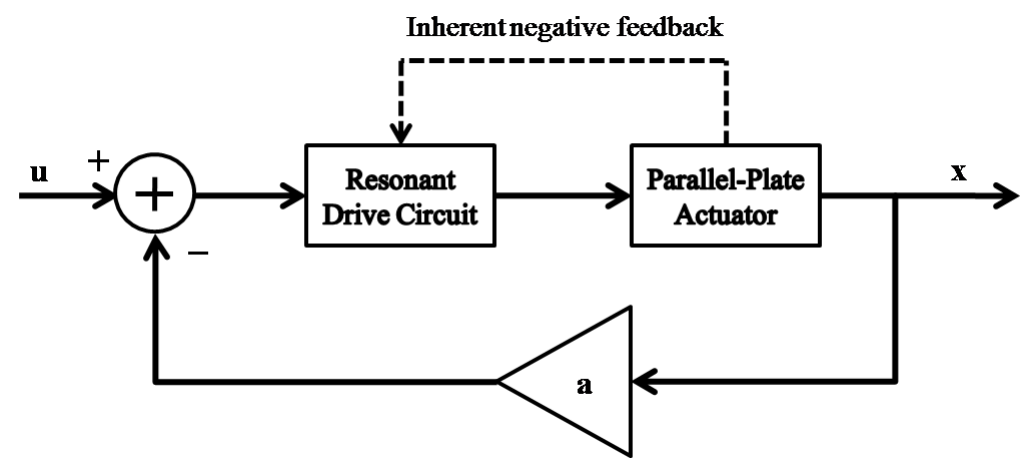

Figure 7.3 Diagram of the feedback controller implemented with the resonant drive circuit that drives the parallel-plate actuator. 
As same in the previous controller, this explicit feedback controller is used to enforce the inherent negative feedback of the resonant drive circuit in order to stabilize the parallel-plate actuator beyond its conventional pull-in point even in the presence of high parasitic capacitance. The non-dimensional governing equation of the parallel-plate actuator with this controller is derived and presented in Eq. 7.2 .

$$
\ddot{x}+2 \xi \omega_{n} \dot{x}+\omega_{m}^{2} x=\frac{2}{27} \omega_{m}^{2} \frac{Q_{f}^{2}(r+1)^{2}(\bar{u}-a x)^{2}}{\left(Q_{f}^{2} x^{2}+(r(1-x)+1)^{2}\right)}
$$

We determine the fixed points of the parallel-plate actuator, dynamics of which is described by Eq. 7.2, and present them in Figure 7.4, where the bottom lines represent the stable fixed points of the parallel-plate actuator with given parasitic capacitances, and the upper lines denote the unstable fixed points of the parallel-plate actuator with the same given parasitic capacitances.

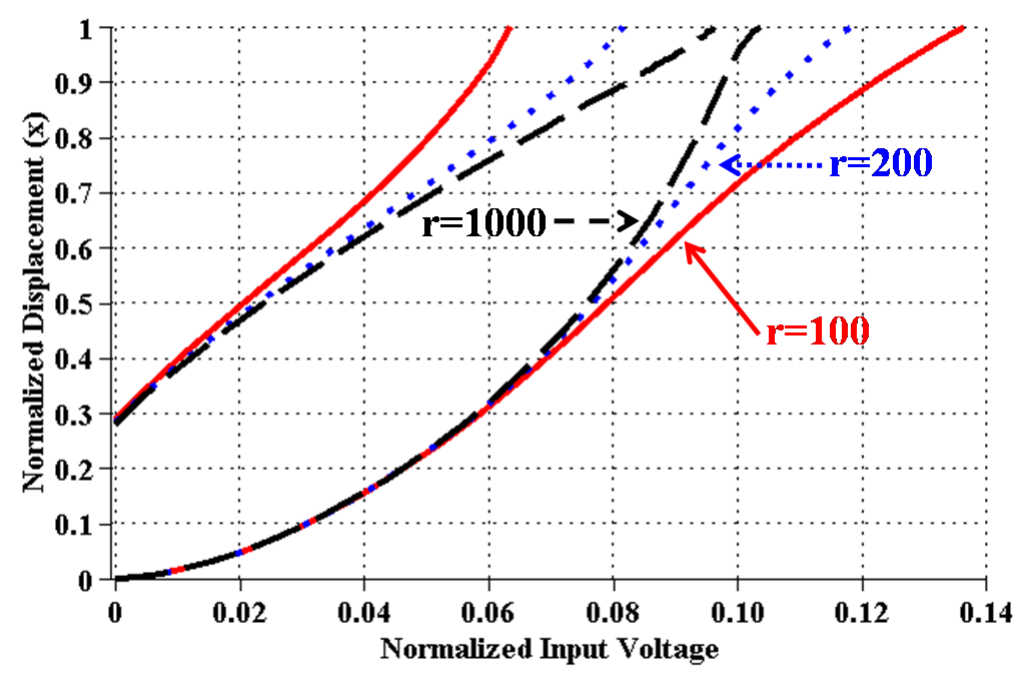

Figure 7.4 Fixed points of the parallel-plate actuator coupled with the resonant drive circuit and the feedback controller in terms of the quality factor $\mathbf{Q}_{\mathrm{f}}=50$, the feedback term of $\mathbf{a}=0.1$, and various parasitic capacitance.

Figure 7.5 presents the fixed points of the parallel-plate actuator with the same controller at three different cases of feedback terms, while the quality factor of the resonant drive circuit is set to be 50 and the parasitic capacitance is 200 times higher than the initial capacitance of the parallel-plate actuator: the red solid lines represent the fixed points at $\mathbf{a}=0.04$; the blue dotted lines denote the fixed points at $\mathbf{a}=0.06$; and the black dashed lines represent the fixed points of the parallel-plate actuator at $\mathbf{a}=0.08$. The upper lines of the same color represent the unstable fixed points, and the bottom lines of the same color denote the stable fixed points. 


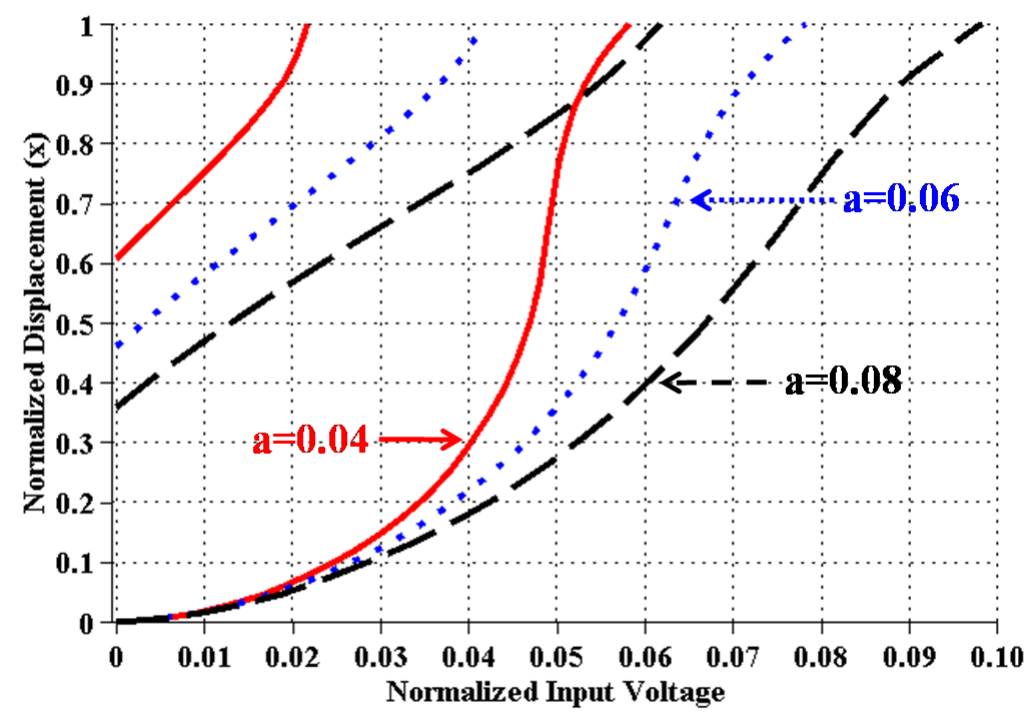

Figure 7.5 Fixed points of the parallel-plate actuator coupled with the resonant drive circuit and the nonlinear controller in terms of the quality factor $\mathbf{Q}_{\mathrm{f}}=50$, the parasitic capacitance $\mathbf{C}_{\mathbf{p}}=200 \mathbf{C}_{\mathbf{0}}$ and various feedback multipliers.

\subsection{Summary}

In this chapter, we present two feedback controllers that are used to stabilize the parallel-plate actuator coupled with the resonant drive circuit, which has a quality factor of 50 . As presented, both feedback controllers are able to extend the operation range of the parallel-plate actuator over its entire gap even in the presence of very high parasitic capacitance. 


\section{Chapter 8}

\section{Conclusions and Future Works}

\subsection{Conclusions}

Most electrostatic actuators require high actuation voltage that must be provided by a high voltage power supply and suffer from the saddle-node bifurcation that limits the operation range of the electrostatic actuators less than their maximum displacement. To overcome these problems, we present various resonant drive circuits that are able to extend the operation range of the electrostatic actuators beyond their conventional pull-in points at much low input voltage even in the presence of high parasitic capacitance. In Chapter 3, we derive and present the analytical and numerical models of the parallel-plate actuator coupled with the resonant drive circuit controlled by both amplitude modulation and frequency modulation in order to determine the fixed points of the parallel-plate actuator and their stability. Furthermore, we also introduce the new displacement measurement method of the electrostatic actuators by sensing the phase delay of the actuation voltage with respect to the input voltage. This displacement measurement method requires neither any physical modification on the electrostatic actuator itself nor any sensing electrodes that increase the complexity and cost of the fabrication. In Chapter 4, we present how the unbalanced and balanced resonant drive circuit are implemented with discrete electronic components and discuss the advantages and disadvantages of each implementation. Moreover, we showcase the resonant drive circuit placed within the positive feedback loop of the non-inverting amplifier in order to enhance the inherent negative feedback of the resonant drive circuit. Its analytical model and numerical models verify that it is capable of driving the parallel-plate actuator over its entire gap in the presence of high parasitic capacitance. In Chapter 5, we create and present the finite element models of various microbeam actuators to prove that the resonant drive circuit is able to drive most electrostatic actuators at much lower input voltage than that of conventional voltage or charge control. Furthermore, the experiment results on the microplate supported by a pair of the microbeams verify the feasibility of the resonant drive circuit. In Chapter 6, we derive and present the analytical and numerical models of the micromirror coupled with the resonant drive circuit. We study its nonlinear dynamics and determine its fixed points and their stability. In addition, the experiment results on the micromirror with the sidewall electrodes are presented to underline the effectiveness of the resonant drive circuit implemented with the H-bridge and complementary MOSFET drivers in terms of the input voltage. In Chapter 7, we present two feedback controllers that make use of the new displacement measurement method introduced in Chapter 3, and prove that the feedback controllers are able to drive the parallel-plate actuator over its entire gap in the presence of high parasitic capacitance.

In addition to these advantages, the resonant drive circuit does not pose any danger to humans and machinery, unlike conventional high voltage actuation circuits that can cause an electric shock when a part of a human body makes a contact with a high voltage section of circuits. Hence, it is inherently

safe and can be safely implemented in many industrial and medical applications where an electric shock must be prevented or avoided. 
As presented and demonstrated in the previous chapters, the resonant drive circuit has the considerable advantages over the conventional voltage or charge control for electrostatic actuation. Its advantages are summarized as thus:

- It does not require a high voltage power supply or a high voltage amplifier to drive the electrostatic actuator, the actuation voltage of which is higher than $100 \mathrm{~V}$.

- It consumes less power than conventional actuation methods because of its low quiescent current and low supply voltage.

- It does not need a heat sink or any other thermal control schemes due to its low power dissipation.

- It can be implemented in a small form factor due to the above mentioned reasons, which is great for portable applications.

- It is inherently safe because high actuation voltage is applied only to the electrostatic actuator and no high voltage or high energy storage component is used.

- It is also innately robust under the worst case scenario due to the inherent negative feedback.

- It is capable of extending the operation range beyond the pull-in point due to the inherent negative feedback in the presence of high parasitic capacitance.

- The measurement of the phase delay of the actuation voltage with respect to the input voltage provides the displacement of the electrostatic actuators without any physical modification on themselves.

- The long-term reliability of the electrostatic actuator with a dielectric layer increases by minimization of the dielectric charging due to the nature of the $\mathrm{AC}$ actuation performed by the resonant drive circuit.

\subsection{Future Works}

Among the research topics and objectives mentioned in Chapter 1, the following topics will be studied further to improve the implementation and performance of the resonant drive circuits.

- New Packaging Method to Reduce Parasitic Capacitance without Chip Carrier

As mentioned in the previous chapters, the parasitic capacitance of the electrostatic actuators is one of the most important factors that determine the performance of the resonant drive circuit. Although the resonant drive circuit is capable of extending the operation range of the electrostatic actuators beyond their conventional pull-in points, the parasitic capacitance undermines its stability and reduces the performance of the resonant drive circuit. Most parasitic capacitances are caused by bonding wires between the silicon die and the chip carrier.

- Resonant Drive Circuit Controlled by Frequency Modulation

As introduced in Chapter 3, the resonant drive circuit controlled by frequency modulation will be studied further, and more experiment will be conducted on various electrostatic actuators coupled with the resonant drive circuit to understand its nonlinear behavior.

- Resonant Drive Circuit Controlled by Phase Modulation 
An analytical and numerical model of the parallel-plate actuator coupled with the resonant drive circuit that is controlled by phase modulation will be studied to determine the robustness of this control method.

- Nonlinear Feedback Controller with Resonant Drive Circuit

A feedback controller is able to drive the electrostatic actuators beyond the pull-in point and improve their performance under random disturbance. The implementation of the feedback controllers described in Chapter 7 will be studied further.

- Dielectric Charging Minimized by Resonant Drive Circuit

The dielectric charging is a big concern on electrostatic actuators with a dielectric layer, because it affects the long term reliability of the electrostatic actuators. We believe the resonant drive circuit is able to minimize this detrimental effect due to the nature of the $\mathrm{AC}$ actuation of the resonant drive circuit. However, it requires the theoretical and experimental validation. 


\section{Bibliography}

[1] Stephen D. Sentruria, “Microsystem Design”, Kluwer Academic Publishers, New York, 2001.

[2] John T.W. Yeow, K. L. Eddie Law, and Andrew A. Goldenberg. "SOI-Based 2-D MEMS L-Switching Matrix for Optical Networking", IEEE Jounal of Selected Topics in Quantum Electronics, pp. 603-613, 2003.

[3] John T.W. Yeow, V. X. D. Yang, A. Chahwan, M. L. Gordon, B. Qi, I. A. Vitkin, B. C. Wilson, A. A. Goldenberg, "Micromachined 2-D scanner for 3-D optical coherence tomography", Sensors and Actuators A:Physical, pp. 331-340, 2005.

[4] Dipika V. Patel, and Charles N.J. McGhee, "Contemporary in vivo confocal microscopy of the living human cornea using white light and laser scanning techniques: a major review", Clin. Experiment. Ophthalmol. 35 (1), pp. 71-88, 2007.

[5] Hiroshi Miyajima, Nobuyoshi Asaoka, Toshihiko Isokawa, Masanori Ogata, Yukihiro Aoki, Masaharu Imai, Osamu Fujimori, Masahiro Katashiro, and Kazuya Matsumoto, "A MEMS electromagnetic optical scanner for commercial confocal laser scanning microscope”, IEEE J. Microelectromech. Syst., vol. 12, no. 3, pp. 243-251, 2003.

[6] Peter F.V. Kessel, Larry J. Hornbeck, Robert E. Meier, and Michael R. Douglass, "A MEMS-Based Projection Display", Proceedings of IEEE, vol. 86, no. 8, August, 1998.

[7] J.I. Seeger and B.E. Boser, "Charge control of parallel-plate, electrostatic actuators and the tip-in instability", J. Microelectromech Syst., vol. 12, no. 5, pp. 656-671, 2003.

[8] J.I. Seeger and S.B. Crary, "Stabilization of electrostatically actuated mechanical devices", Interneational Conference on Solid-State Sensors and Actuator, vol. 2, pp. 1133-1136, 1997.

[9] E. K. Chan and R. W. Dutton, "Electrostatic micromechanical actuator with extended range of travel", J. Microelectromech Syst., vol. 9, pp. 321-328, 2000.

[10]D. H. S. Maithripala, J. M. Berg and W. P. Dayawansa, "Capacitive stabilization of an electrostatic actuator: Output feedback viewpoint", American Control Conference 2003, vol. 5, pp. 4053-4058, 2003.

[11]C. G. Agudelo, M. Packirisamy, G. Zhu and L. Saydy, "Nonlinear control of an electrostatic micromirror beyond pull-in with experimental validation", J. Microelectromech Syst, vol. 18, pp. 914-923, 2009.

[12] K. O. Owusu and F. L. Lewis, "Solving the pull-in instability problem of electrostatic microactuators using nonlinear control techniques", the 2nd IEEE International Conference on Nano/Micro Engineered and Molecular Systems, pp. 1190-1195, 2007.

[13]D. Piyabongkarn, Y. Sun, R. Rajamani, A. Sezen and B. J. Nelson, "Travel range extension of a MEMS electrostatic microactuator", IEEE Trans. Control Syst. Technol., vol. 13, pp. 138-145, 2005. 
[14] Takayuki Iseki, Miki Okumura and Takashi Sugawara, "Two-Dimensionally Deflecting Mirror Using Electromagnetic Actuation", Optical Review, pp. 189-194, 2006.

[15] Orphee Cugat, Jerome Delamare, and Gilbert Reyne, "Magnetic Micro-Actuators and Systems (MAGMAS)", IEEE Transactions on Magnetics, pp. 3607-3612, 2003.

[16] M R J Gibbs, E W Hill and P J Wright, "Magnetic materials for MEMS applications", Journal of Physics D:Applied Physics, pp. R237-R244, 2004.

[17] Jonathan J. Bernstein, William P. Taylor, John D. Brazzle, Christopher J. Corcoran, Gregory Kirkos, Jefferson E. Odhner, Ajay Pareek, Marc Waelti, and Marvin Zai, "Electromagnetically Actuated Mirror Arrays for Use in 3-D Optical Switching Applications", Journal of Microelectromechanical Systems, pp. 526-535, 2004.

[18] Chuan Pu, Sangtae Park, Patric B. Chu, Shi-Sheng Lee, Ming Tsai, David Peale, Nico H. Bonadeo, and Igal Brener, "Electrostatic actuation of three-dimensional MEMS mirrors using sidewall electrodes", IEEE Journal of Selected Topics in Quantum Electronics, vol. 10, no. 3, pp. 472-477, 2004.

[19] Jeffery F. Rhoads, Steven W. Shaw, and Kimberly L. Turner, "The nonlinear response of resonant microbeam systems with purely-parametric electrostatic actuation", Journal of Micromechanics and Microengineering, pp. 890-899, 2006.

[20] Janak Singh, Terence Gan, Ajay Agarwal, Mohanraj, and Saxon Liw, "3D free space thermally actuated micromirror device", Sens. Actuators A, pp.468-475, 2005.

[21] Amarendra Atre, "Analysis of out-of-plane thermal microactuators", Journal of Micromechanics and Microengineering, pp. 250-213, 2006.

[22] Tatsuo Kawabata, Masaaki Ikeda, Hiroshi Goto, Mikio Matsumoto, and Yada Tsuneji, "The 2-Dimentional micro scanner integrated with PZT thin film actuator", IEEE International Conference on Solid-State Sens. Actuators, pp. 339-342, 1997.

[23] Robbins, William P. "High-Displacement Piezoelectric Actuator Utilizing a Meander-Line Geometry-Part II:Therory", IEEETransactions on Ultrasonics, Ferroelectrics, and Frequency Control, pp. 461-467, 1991.

[24] Young Ho Seo, Doo-Sun Choi, Joon-Hyung Lee, Taik-Min Lee, Tae-Jin Je and Kyung-Hyun Whang. "Piezoelectric Actuator Based on Stiffness Control and Stroke Amplication for Large Lateral Actuation", IEEE International Conference on Micro Electro Mechanical Systems, pp. 383-386, 2005.

[25]H. Camon, C. Ganibal, "Advantages of alternative actuating signal for MEMS actuators", Sensors and Actuators A:Physical 136, pp. 299-303, 2007.

[26] J. M. Kyynäräinen, A. S. Oja, H. Seppa, "Increasing the Dynamic Range of a Micromechanical MovingPlate Capacitor", Analog Integrated Circuits and Signal Processing 29, pp. 61-70, 2001. 
[27] Baris Cagdaser and Bernhard E. Boser, "Resonant drive for stabilizing parallel-plate actuators beyond the pull-in point", the 13th International Conference on Solid-State Sensors Actuators and Microsystems Transducers'05, vol. 1, pp. 688-692, 2005.

[28] Robert C. Anderson, Balasaheb Kawade, Kandiah Raulan, D.H.S. Maithripala, Jordan M. Berg, Richard O. Gale, and W.P. Dayawansa, "Integrated charge and position sensing for feedback control of electrostatic MEMS”, Proc. Smart Structures and Materials, San Diego, USA, March 2005.

[29] W. Merlijn van Spengen, and Tjerk H. Oosterkamp, "A sensitive electronic capacitance measurement system to measure the comb dirve motion of surface micromachined MEMS devices", J. Micromech. Microeng. 17, pp. 828-834, 2007.

[30] Neal A. Hall, Murat Okandan, Robert Littrell, Darwin K. Serklan, Gordon A. Keeler, Ken Peterson, Baris Bicen, Caesar T. Garcia and F. Levent Degertekin, "Micromachined Accelerometers With Optical Interferometric Read-Out and Integrated Electrostatic Actuation", Journal of Microelectromechanical Systems, vol. 17, no. 1, Feb., 2008.

[31] Assaf Ya'akobovitz, Slava Kryov, and Yael Haneib, "Nanoscale displacement measurement of electrostatically actuated micro-devices using optical microscopy and digital image correlation", Sens. Actuators A:Phys. 162, pg. 1-7, 2010.

[32] S. Towfighian, G.R. Heppler, E. M. Abdel-Rahman, "Analysis of a chaotic electrostatic Micro-oscillator", Journal of Comutational and Nonlinear Dynamicis, vol. 6, no. 1, January, 2011.

[33] S. Towfighian, G.R. Heppler, E. M. Abdel-Rahman, "Dynamic A low voltage controller for a chaotic micro resonantor”, Proceedings of the ASME 2010 Internaltional DETC, Montreal, Canada, August, 2010.

[34] K.G. Ong, C.A. Grimes, C.L. Robbins, and R.S. Singh, "Design and applicaton of a wireless, passive, resonant-circuit environmetal monitoring sensor", Sens. Actuators A:Phys. 93, pp. 33-43, 2001.

[35] R.A. Potyrailol, W.G. Morris, T. Sivavec, H.W. Tomlinson, S. Klensmeden, and K. Lindh, "RFID sensors based on ubiquitous passive 13.56-MHz RFID tags and complex impedance detection", Wireless Communications and Mobile Computing, vol. 9, pp. 1318-1330, 2009.

[36]P.-J. Chen, S. Saati, R. Varma, M.S. Humayun, and Y.-C. Tai, "Wireless intraocular pressure sensing using microfabricated minimally invasive flexible-coiled LC sensor implant", Journal of Microelectromechanical Systems, vol. 19, pp. 721-734, 2010

[37] S. Liu, R. Farrow, J.L. Zunino III, H.C. Lim, J. Federici, and G. Thomas, "Microfabricated implantable pressure sensor for flow measurement", Proc. of SPIE, 2008.

[38] G. Stojanović, M. Radovanović, M. Malešev, and V. Radonjanin, "Monitoring of water content in building materials using a wireless passive sensor”, Sens. Actuators A:Phys. 10, pp. 4270-4280, 2010. 
[39] M. Yvanoff, J. Venkataraman, “A feasibility study of tissue characterization using LC sensors", IEEE Transcactions on Antennas and Propagation, pp. 885-893, 2009.

[40]D. Marioli, E. Sardini, and M. Serpelloni, "An inductive telemetric measurement systesm for humidity sensing", Measurement Science and Technology, art. no. 115204, 2008.

[41] J. García-Cantón, A. Merlos, and A. Baldi, “A wireless LC chemical sensor based on a high quality factor EIS capacitor", Sens. Actuators B:Chem. 126, pp. 648-654, 2007.

[42] C.-Y. Kim, I.I.-B. Kwon, and D.-C. Seo, "Wireless instrumentation for monitoring of smart structures", Key Engineering Materials, pp. 192-195, 2006.

[43] Steven H. Strogatz, "Nonlinear Dynamics and Chaos:with applications to Physics, Biology, Chemistry, and Engineering”, pp. 215-227, Westview Press, New York, 2001.

[44] Alexander S. Kutyrev, Richard Arendt, S. Harvey Moseley, Ray A. Boucarut, Theo Hadjimichael, Murzy Jhabvala,Todd King, Mary J. Li, James Loughlin, David Rapchun, David Scott Schwinger, and R. F. Silverberg, "Programmable Microshutter Arrays for the JWST NIRSpec: Optical Performance", IEEE Journal of Selected Topics in Quantum Electronics, vol. 10, no. 3, 2004.

[45] J. I. Seeger and B. E. Boser, "Negative capacitance for control of gap-closing electrostatic actuators", the 12th International Conference on Solid-State Sensors, Actuators and Microsystems, vol.1, pp. 484-487, 2003.

[46] S. Bermejo and L. Castañer, "Dynamics of MEMS electrostatic driving using a photovoltaic source", Sensors and Actuators A: Physical, vol. 121, pp. 237-242, 2005.

[47] Giuseppe Barillaro, Antonio Molfese, Andrea Nannini, and Francesco Pieri, "Analysis, simulation and relative performances of two kinds of serpentine springs", Journal of Micromechnics and Microengineering, pp. 736-746, 2005.

[48]Jianliang You, Muthukumaran Packirisamy, and Ion Stiharu, "Analysis, Simulation and Testing of a Micromirror with Rotational Serpentine Springs", Intelligent Sensing and Information Processing, pp. 219$225,2005$.

[49] Robert Sattler, Florian Plötz, Gernot Fattinger, and Gerhard Wachutka, "Modeling of an electrostatic torsional actuator: demonstrated with an RF MEMS switch”, Sens. Actuators A 97-98, pp. 337-346, 2002.

[50] Sangtak Park, Yanhui Bai, and John T.W. Yeow, "Design and Analysis of Resonant Drive Circuit for Electrostatic Actuators", International Symposium on Optomechatronic Technologies 2010, Toronto, Canada, October, 2010.

[51] Joao Ramos and M.S.J. Steyaert, "Positive feedback frequency compensation for low-voltage low-power three-stage amplifier", IEEE Transactions on Circuits and Systems I, vol. 51, pp. 1967-1974, 2004. 
[52] M. Pude, P.R. Mukund, P. Singh, K. Paradis and J. Burleson, "Amplifier gain enhancement with positive feedback", IEEE International Midwest Symposium on Circuits and Systems (MWSCAS) 2010, pp. 981984, 2010.

[53] M.E. Schlarmann, S.Q. Malik and R.L. Geiger, "Positive feedback gain-enhancement techniques for amplifier design", IEEE International Symposium on Circuits and Systems 2002, vol. 2, pp. II-37-II-40, 2002.

[54] Paul Horowitz and Winfield Hill, "The art of electroncis", Cambridge Univeristy Press, pp. 177-183, pp. 263-303, $2^{\text {nd }}$ edition, New York, 1989.

[55]Eihab M. Abdel-Rahman, Mohamed I. Younis, and Ali H. Nayfeh, "Characterization of the machnaical behaviour of an electrically actuated microbeam", Journal of Miceomechanics and Microengineering, vol. 12, no. 6, pp. 759-766, 2002. 